\title{
The role and regulation of histone H2B monoubiquitination during tumorigenesis
}

\author{
Dissertation \\ for the award of the degree \\ "Doctor rerum naturalium (Dr. rer. nat.)" \\ Division of Mathematics and Natural Sciences \\ of the Georg-August-Universität Göttingen
}

\author{
submitted by \\ Theresa Gorsler \\ born in Leinefelde
}

Hamburg, 2013 
Members of the Thesis Committee:

Prof. Dr. Steven A. Johnsen (Reviewer)

Department of Tumor Biology

University Medical Center Hamburg-Eppendorf, Hamburg

Prof. Dr. Holger Reichardt (Reviewer)

Department of Cellular and Molecular Immunology

University of Göttingen Medical School, Göttingen

Prof. Dr. Dieter Kube

Department of Immunology and Experimental Oncology

University of Göttingen Medical School, Göttingen

Date of the oral examination: $3^{\text {rd }}$ of june 2013 


\section{Affidavit}

I hereby declare that the $\mathrm{PhD}$ thesis entitled "The role and regulation of histone $\mathrm{H} 2 \mathrm{~B}$ monoubiquitination during tumorigenesis" has been written independently and with no other sources and aids than quoted.

Theresa Gorsler

April, 2013

Hamburg 


\section{Table of Contents}

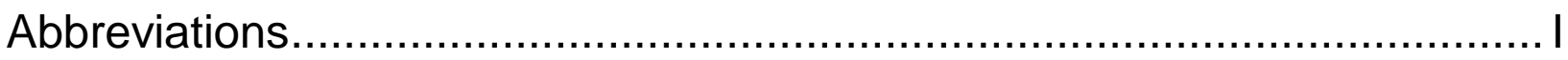

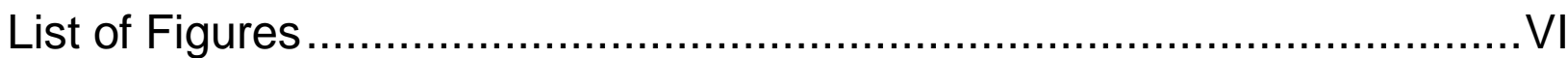

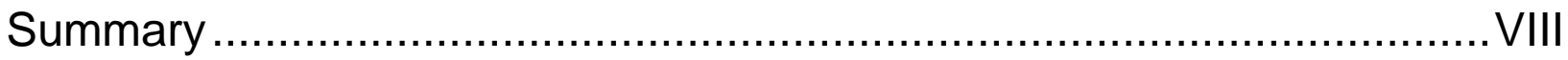

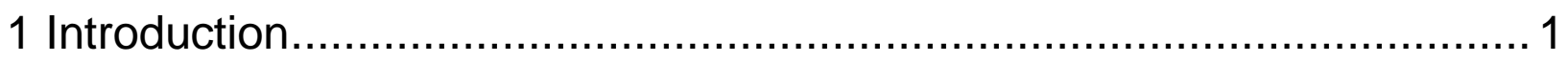

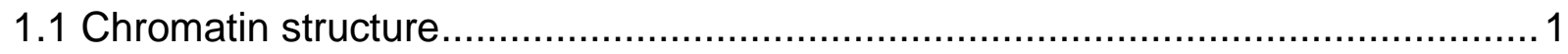

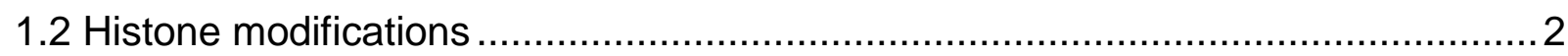

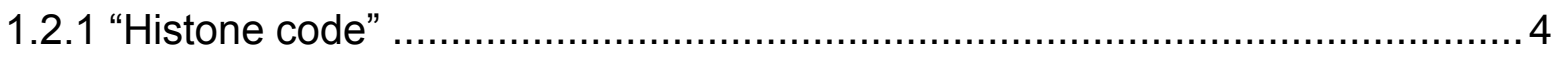

1.2.2 Misregulation of histone modifications ...................................................... 5

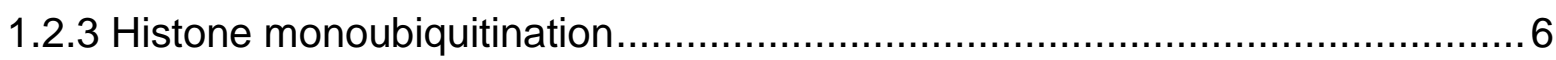

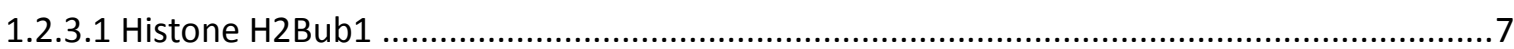

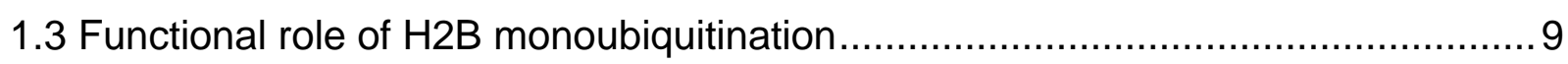

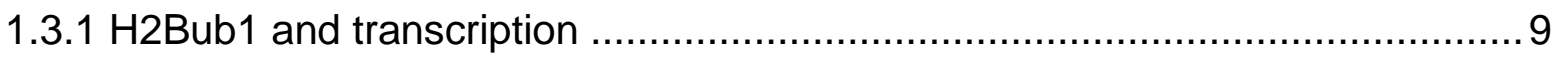

1.3.2 Cellular functions of the H2Bub1 in yeast ............................................... 10

1.3.3 The role of H2Bub1 in higher eukaryotes...............................................11

1.3.4 Genome-wide distribution of H2Bub1 .............................................. 13

1.3.5 H2Bub1 and its regulation in human cancer ............................................ 14

1.4 Histone H2B deubiquitination ................................................................ 14

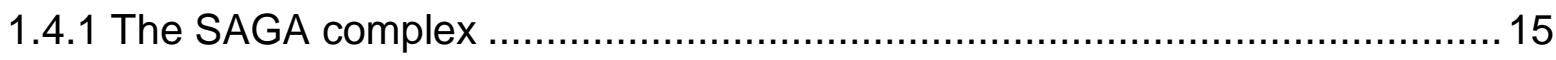

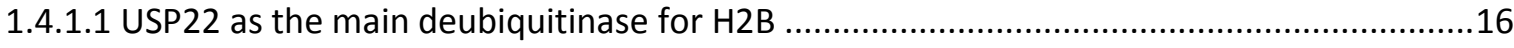

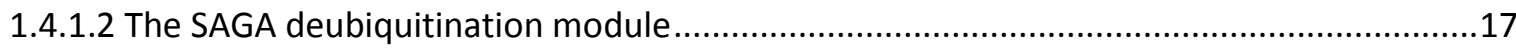

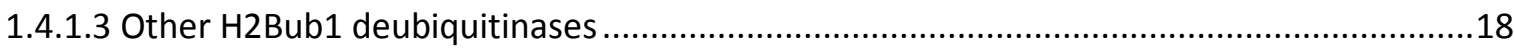

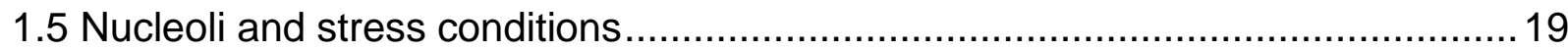

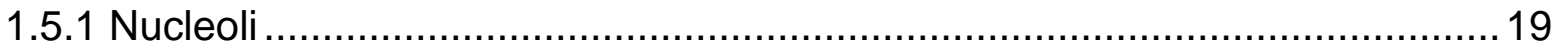

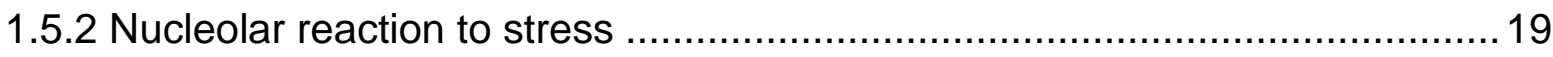

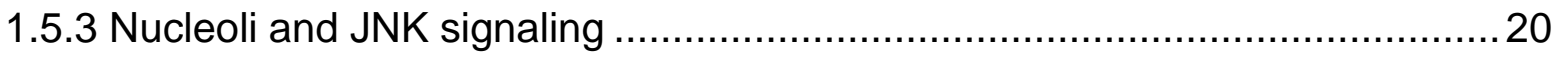

1.5.4 AMPK signaling in tumorigenesis and tumor metabolism ..........................21 


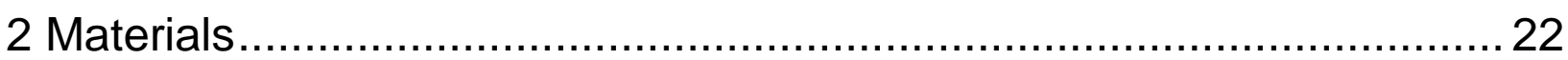

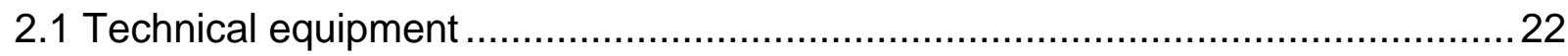

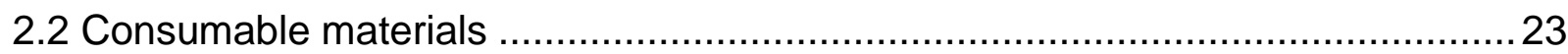

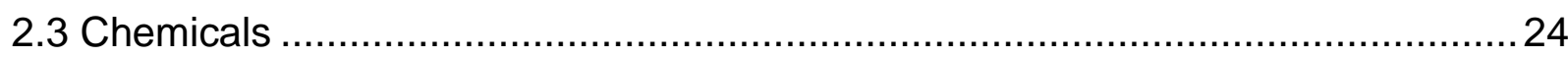

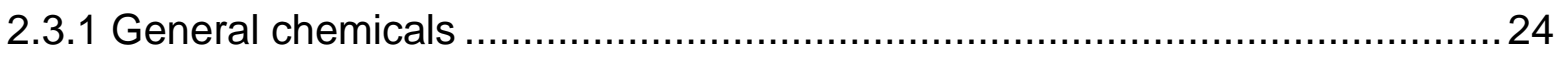

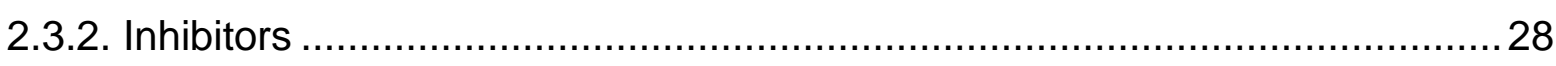

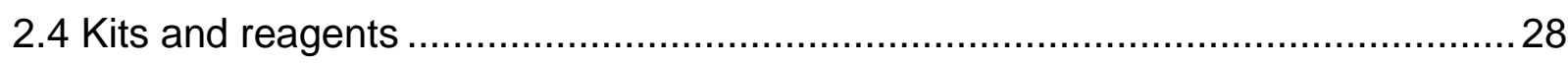

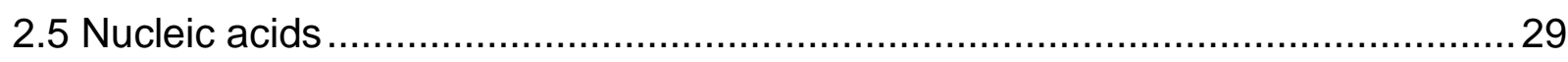

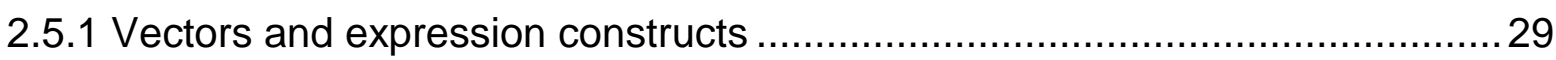

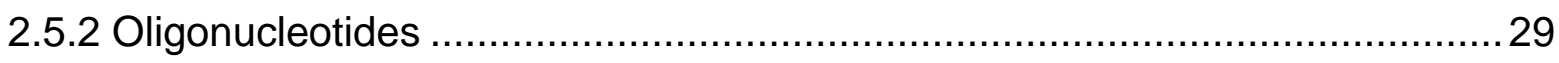

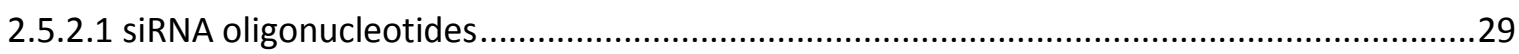

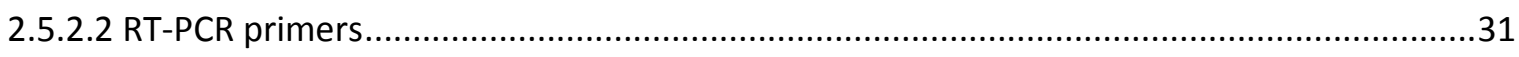

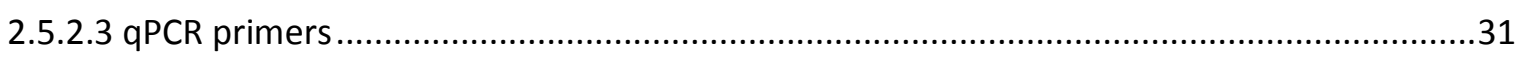

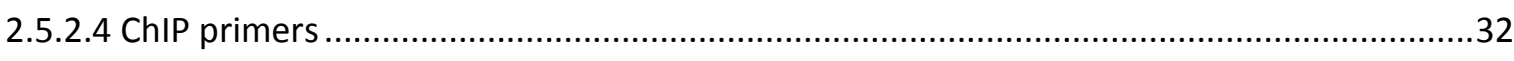

2.5.2.4 Primers for ChIP-seq library preparation .................................................................32

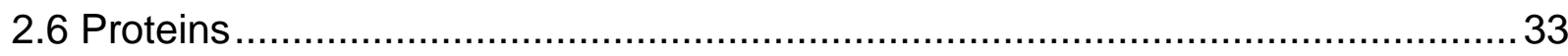

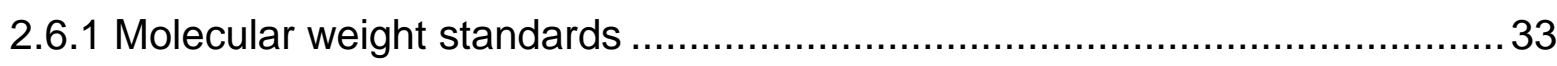

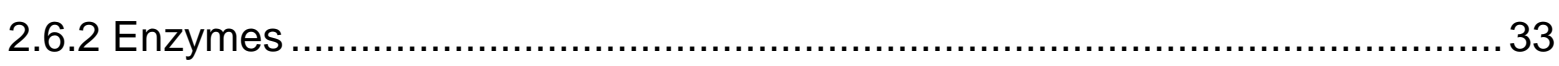

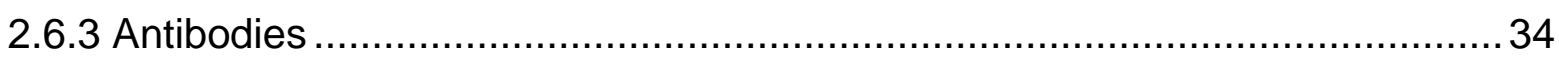

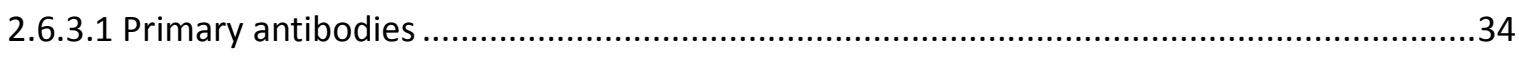

2.6.3.2 Secondary antibodies .................................................................................................

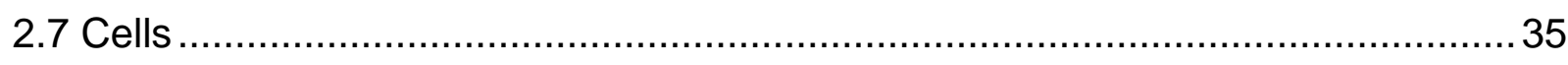

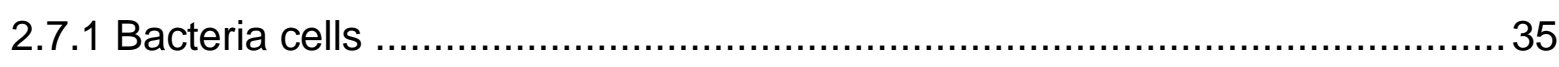

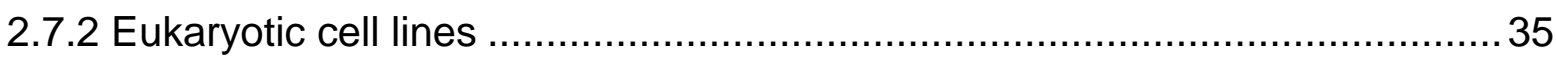

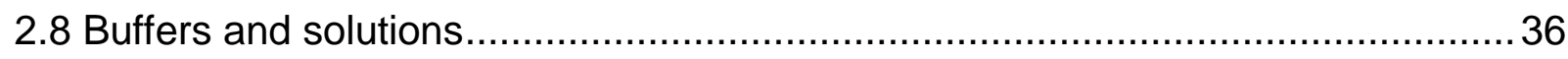

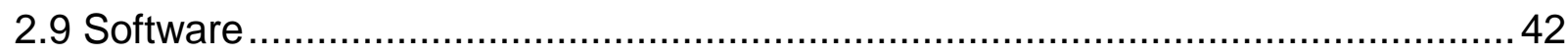

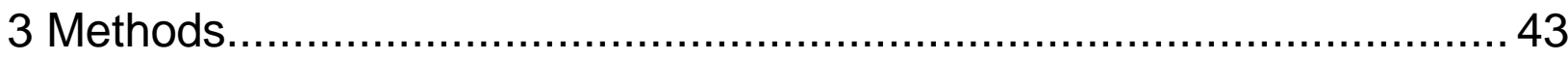

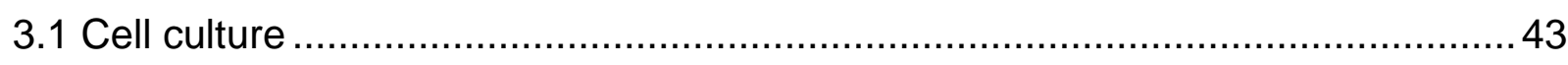

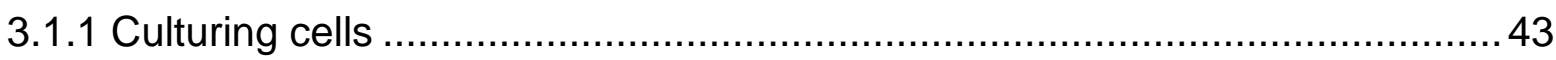




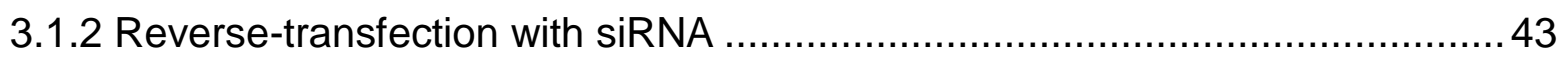

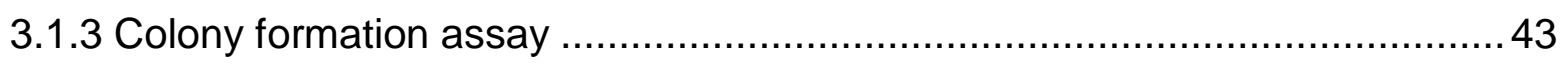

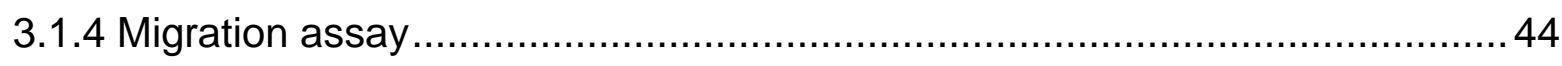

3.1.5 Measurement of DNA of single cells by flow cytometry ................................ 44

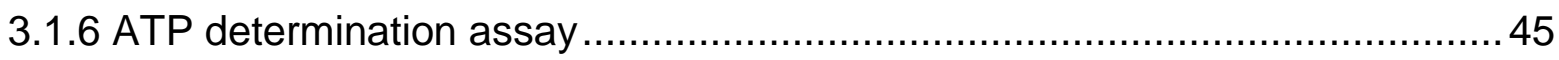

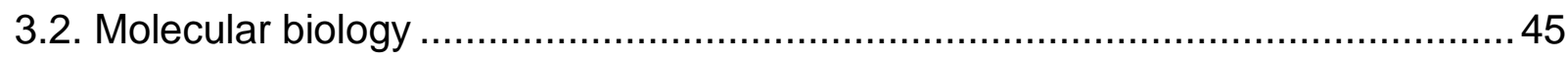

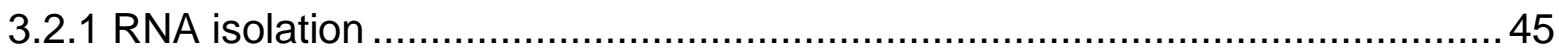

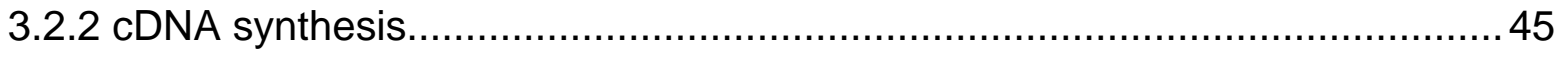

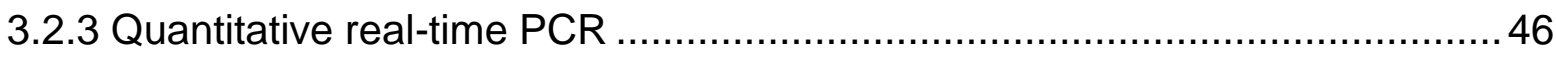

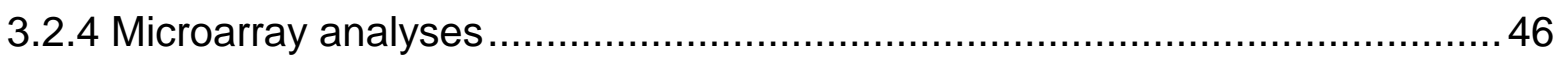

3.2.5 Chromatin immunoprecipitation (ChIP) ………................................. 48

3.2.6 Chromatin immunoprecipitation-sequencing (ChIP-Seq) …........................ 49

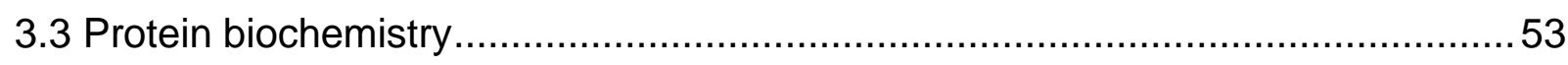

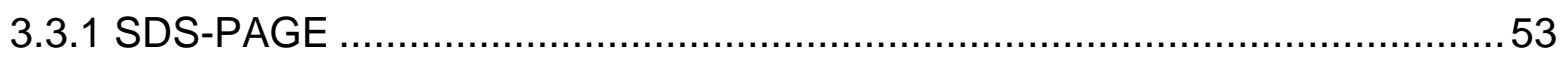

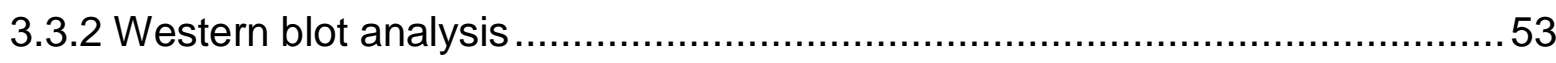

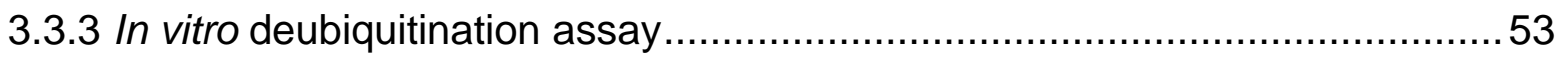

3.3.4 Immunohistochemistry on paraffin sections ……....................................54

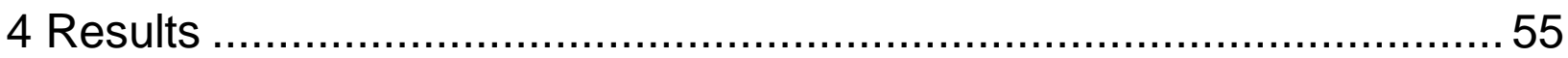

4.1 H2B ubiquitinating enzymes affect cellular responses ……............................55

4.1.1 Effects of RNF40 depletion on proliferation of breast cancer and

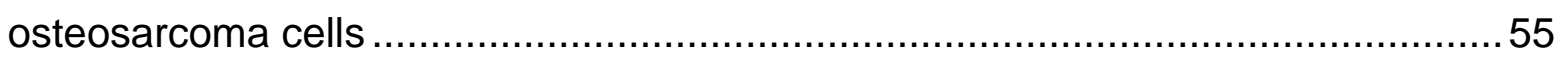

4.1.2 RNF20 and RNF40 depletion leads to a cell cycle arrest............................57

4.1.3 Effects of RNF40 overexpression on cell proliferation ................................58

4.1.4 RNF40 overexpression induces a G2/M arrest in osteosarcoma cells...........60

4.1.5 RNF20 and RNF40 similarly suppress migratory potential ...........................61

4.1.6 Messenger RNA expression profiling of RNF20 and RNF40-regulated genes

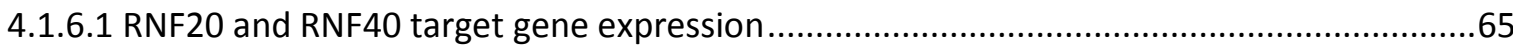

4.1.7 H2Bub1 decreases during tumor progression.........................................6 66 
4.1.8 Genomic regulation of ATXN7L3 and USP22 in breast cancer

4.2 H2Bub1 levels decrease following various stress conditions 69

4.2.1 Different stress factors rapidly reduce H2B monoubiquitination 69

4.2.2 Treatment with stress-inducing factors alters RNF20- and RNF40- regulated gene transcription ................................................................................... 71

4.2.2.1 Actinomycin D affects rRNA transcription 72

4.2.2.2 Actinomycin D concentration specifically affects RNF20 and RNF40 target gene transcription .74

4.2.2.3 Cisplatin affects H2Bub1 occupancy .75

4.2.2.4 RNAPI inhibitor decreases H2Bub1 level similar to Actinomycin D .76

4.3 Cellular stress activates several signaling pathways...................................... 77

4.3.1 JNK activity regulates the level of H2B ubiquitination ................................77

4.3.2 Regulation of H2B ubiquitination upon AMP signaling .............................. 78

4.3.2.1 AICAR treatment leads to loss of H2Bub1 levels..............................................................

4.3.2.2 The effect of upstream kinases on H2Bub1 level...........................................................79

4.3.2.3 Effect of AICAR on RNF20 and RNF40 target gene transcription ......................................81

4.3.2.4 AICAR affects H2Bub1 occupancy of RNF20/RNF40 target genes ....................................82

4.3.2.5 Involvement of the glucose metabolism in regulating global H2Bub1 levels .....................83

4.4 H2Bub1 levels are reduced due to the activation of a cellular deubiquitinating

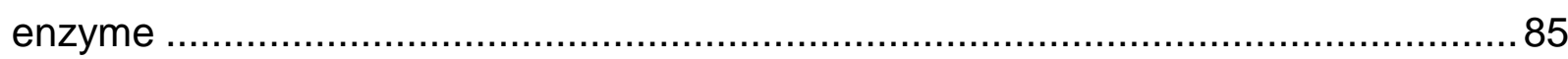

4.4.1 In vitro deubiquitination assay showed increased deubiquitinating activity upon treatment with stress-inducing factors................................................................. 85

4.4.2 The SAGA complex is required for H2B deubiquitination............................. 86

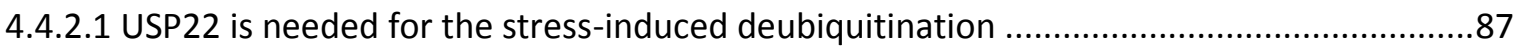

4.4.2.2 Chemotherapeutical treatment also affects $\mathrm{H} 2 \mathrm{~A}$ ubiquitination ....................................91

4.4.2.3 Depletion of SAGA DUB module components leads to H2Bub1 accumulation ..................92

4.5 Genome-wide analysis of H2Bub1 distribution ................................................97

4.5.1 ChIP-seq analyses show H2Bub1 occupancy and its changes upon ATXN7L3

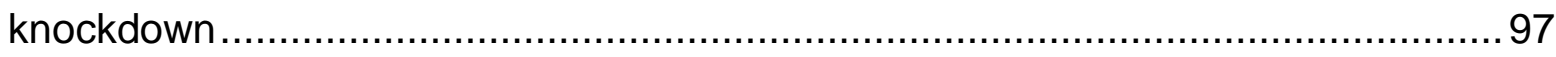

4.5.2 Genome-wide averages of the H2Bub1 profile based on gene expression level 101

5 Discussion 103 
5.1 The role of H2Bub1 as a potential tumor suppressor ................................ 103

5.1.1 Function of RNF20 and RNF40 in cellular responses .......................... 103

5.1.2 RNF20 and RNF40 modulated gene expression ............................ 106

5.1.3 Substrate specificity of RNF20 and RNF40 ................................... 109

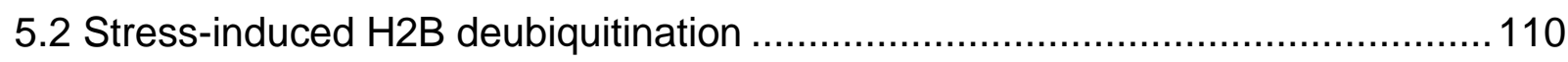

5.2.1 H2Bub1 is strongly reduced by various treatments ............................ 110

5.2.2 JNK and nucleolar signaling are involved in reduction of $\mathrm{H} 2 \mathrm{Bub1} 1 . . . \ldots \ldots \ldots . . . . .111$

5.2.3 Metabolic regulation of H2Bub1 levels .......................................... 112

5.3 Activation of a cellular deubiquitinating enzyme reduces H2Bub1 levels ...........113

$5.4 \mathrm{H} 2 \mathrm{Bub1}$ as a therapeutic target in cancer ............................................. 115

5.5 Genome-wide distribution of H2Bub1 .............................................. 118

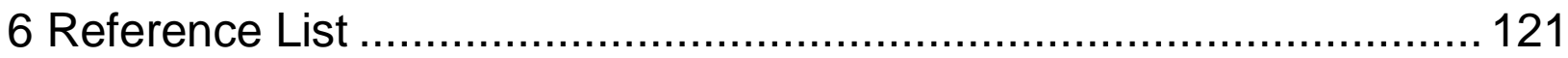

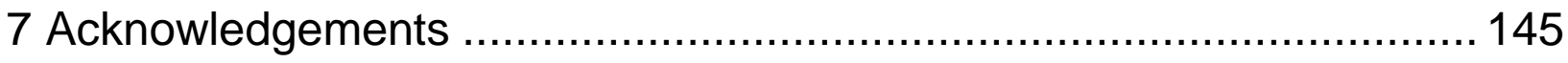

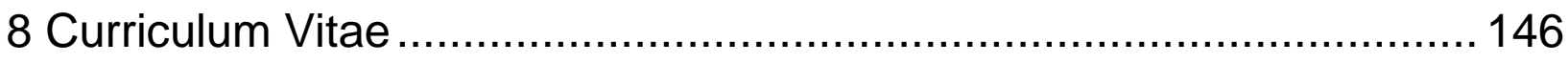




\section{Abbreviations}

${ }^{\circ} \mathrm{C}$

2-DG

53BP1

ABCC2

AICAR

AMP

AMPK

APS

ATM

ATP

ATR

ATXN7L3

BGP

BMI-1

BRCA1

Brd4

Bre1

CDK

cDNA

ChIP

ChIP-Seq

$\mathrm{CO}_{2}$

COMPASS

Cont

$\mathrm{CpG}$

CTD

$\mathrm{ddH}_{2} \mathrm{O}$
Degree Celsius / centigrade

2-Deoxy-D-glucose

p53 binding protein 1

ATP-binding cassette, sub-family $C$

Aminoimidazol-Carboxamid-Ribonukleosid

Adenosinmonophosphate

adenine monophosphate-activated protein kinase

Ammonium peroxysulfate

Ataxia telangiectasia mutated

Adenosin triphosphate

Ataxia telangiectasia and Rad3 related

Spt-Ada-Gcn5-acetyltransferase

B-Glycerolphosphate

B lymphoma Mo-MLV insertion region 1 homolog

Breast cancer 1

Bromodomain containing 4

Brefeldin A sensitivity

Cyclin-Dependent Kinase

Complementary DNA

Chromatin immunoprecipitation

ChIP followed by high-throughput sequencing

Carbon dioxide

Complex proteins associated with Set1p

Control

Cytosin phosphat Guanin

Carboxyterminal Domain

Double distilled water 
DEPC

DMEM

DMSO

DNA

Dot1L

DSB

DTT

DUB

DUBm

e.g.

E1 enzyme

E2 enzyme

E3 enzyme

Ebp1

EDTA

EGF

EMT

ENY2

ERK

$\mathrm{EtOH}$

$\mathrm{F}$

FACS

FACT

FBS

FST

GAPDH

GCN5

h
Diethylpyrocarbonate

Dulbecco/Vogt modified Eagle's minimal essential medium

Dimethyl sulfoxide

Deoxyribonucleic acid

DOT1-like

Double-strand break

Dithiothreitol

Deubiquitinating enzyme

Deubiquitination module

Exempli gratia $=$ for example

Ubiquitin-activating enzyme

Ubiquitin-conjugating enzyme

Ubiquitin-ligase

ErbB3-associated protein

Ethylenediaminetetraacetic acid

Epidermal growth factor

Epithelial-mesenchymal transition

Enhancer of yellow 2 homolog

Extracellular signal-regulated kinases

Ethanol

Forward

Fluorescence-Activated Cell Sorting

Facilitates Active Chromatin Transcription

Fetal Bovine Serum

Follistatin

Glycerinaldehyd-3-phosphat-Dehydrogenase

Histone acetyltransferase, general control of amino acid synthesis protein 5

Hour 
$\mathrm{H} 2 \mathrm{~A}$

H2Aub1

H2B

H2Bub1

$\mathrm{H} 3$

$\mathrm{H} 4$

hMSCs

hnRNA

HoxA

HRP

IAA

$\lg G$

IL1B

IL1RAP

JNK

K

$\mathrm{kDa}$

L

LKB1

m

M

MAPK

MDM2

me

MEKK1

MET

MG132

$\min$

MLL
Histone 2A

Monoubiquitinated histone $2 \mathrm{~A}$

Histone 2B

Monoubiquitinated histone $2 \mathrm{~B}$

Histone 3

Histone 4

human mesenchymal stem cells

Heterogeneous nuclear RNA

Homeobox A cluster

Horseradish peroxidase

lodacetamide

Immunoglobulin G

Interleukin-1 beta

Interleukin-1 receptor accessory protein

C-Jun N-terminal kinase

Lysine residue

Kilo Dalton

Leucine residue or liter

Liver Kinase B1

Milli (10-3)

Methionine residue or molar, $\mathrm{mol} / \mathrm{L}$

Mitogen-activated protein kinases

Mouse double minute 2 homolog

methylation

Mitogen-activated protein kinase kinase kinase

Mesenchymal-epithelial transition

Carbobenzoxy-L-leucyl-L-leucyl-L-leucinal

Minute

Myeloid/lymphoid or mixed-lineage leukemia 


\begin{tabular}{|c|c|}
\hline mRNA & messenger RNA \\
\hline MYBBP1A & MYB binding protein (P160) 1a \\
\hline $\mathrm{n}$ & Nano (10-9) \\
\hline$n$ & number of individual values \\
\hline n.s. & non-significant \\
\hline NCL & Nucleolin \\
\hline NELF & Negative elongation factor \\
\hline NEM & $\mathrm{N}$-ethylmaleimide \\
\hline NP-40 & Nonidet P40 \\
\hline $\mathrm{P} / \mathrm{S}$ & Penicillin/streptomycin \\
\hline PAF & RNA Polymerase II Associated Factor \\
\hline PBS & Phosphate Buffered Saline \\
\hline PBS-T & Phosphate Buffered Saline with Tween-20 \\
\hline PcG & Polycomb group \\
\hline PCNA & Proliferating Cell Nuclear Antigen \\
\hline $\mathrm{pH}$ & Measurement of acidity or alkalinity of a solution \\
\hline $\mathrm{PI}$ & Propidium lodide \\
\hline PRC & Polycomb repressive complex \\
\hline P-TEFb & Positive Transcription Elongation Factor beta \\
\hline PTM & Posttranslational modification \\
\hline $\mathrm{R}$ & Reverse \\
\hline Rad6 & Radiation sensitivity protein 6 \\
\hline RING & Really Interesting New Gene \\
\hline RNA & Ribonucleic acid \\
\hline RNAPII & RNA Polymerase II \\
\hline RNF & Ring finger protein \\
\hline RPLP0 & Ribosomal protein, large, P0 \\
\hline rRNA & Ribosomal ribonucleic acid \\
\hline RT & Room Temperature \\
\hline
\end{tabular}


RT-PCR

\section{S}

S

s.d.

SAGA

SAPKs

SDS

SDS-PAGE

siRNA

miRNA

IncRNA

SP600125

Taq

TEMED

TFIIS

TGF- $\beta$

TIF-IA

TREX-2

Tris

TTS

UBE2

UBP

USP22

WAC

WB

$\mu$
Reverse Transcription PCR

Second

Serine residue

Standard deviation

Spt-Ada-Gcn5-Acetyltransferase

Stress-activated protein kinases

Sodium dodecylsulfate

Sodium dodecylsulfate polyacrylamide gel electrophoresis

Small interfering ribonucleic acid

Micro-ribonucleic acid

Long non-coding RNA

1,9-Pyrazoloanthrone

Thermus aquaticus

Tetramethylethylenediamine

Transcription factor II S

Transforming growth factor-beta

Transcription initiation factor IA

Three prime repair exonuclease 2

Tris(hydroxymethyl)aminomethane

Transcription terminating site

Ubiquitin-conjugating enzyme E2

Ubiquitin protease

Ubiquitin Specific Peptidase 22

WW domain containing adaptor with coiled-coil region

Western Blot

Micro (10-6) 


\section{List of Figures}

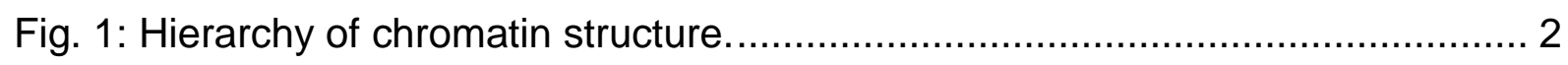

Fig. 2: Main posttranslational histone modifications. ............................................ 4

Fig. 3: H2Bub1 regulation via the CDK9-WAC-RNF20/RNF40 axis. ..................... 10

Fig. 4: The SAGA deubiquitination module is conserved from yeast to humans. .... 17

Fig. 5: siRNA-mediated knockdown of RNF40 increased colony formation in MCF10A

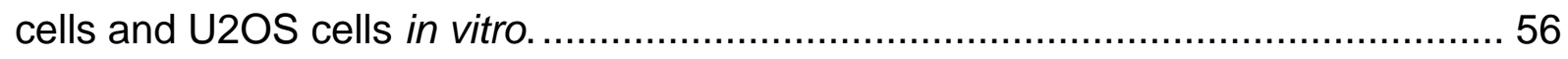

Fig. 6: Knockdown of RNF20 and RNF40 increases the percentage of MCF10A cells

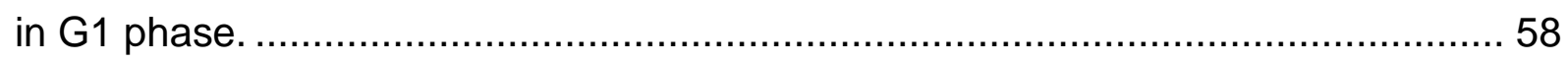

Fig. 7: Ectopic expression of RNF40 inhibits tumor cell growth. ........................... 59

Fig. 8: RNF40 overexpression increases the percentage of U2OS-Tet-RNF40 cells in

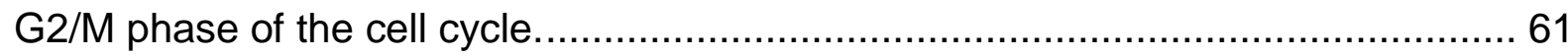

Fig. 9: Cell migration assay after RNF20 or RNF40 knockdown............................ 62

Fig. 10: mRNA expression profiling of RNF20- and RNF40-regulated genes.......... 64

Fig. 11: qRT-PCR validation of selected genes from the microarray experiment. .... 66

Fig. 12: H2Bub1 and RNF40 expression in human normal colon tissue and tumors. 67

Fig. 13: Genomic regulation of ATXN7L3 and USP22 in breast cancer. ................. 69

Fig. 14: Various factors down-regulate H2B ubiquitination. ................................. 70

Fig. 15: Different stress factors decrease RNF20 and RNF40 target gene expression.

Fig. 16: H2Bub1 reduction happens very rapidly and affects rRNA transcription. .... 73

Fig. 17: RNF20 and RNF40 target gene expression but not general RNAPII transcription is affected upon Actinomycin D treatment....................................... 74

Fig. 18: Chromatin immunoprecipitation (ChIP) analysis revealed a decrease in H2Bub1 occupancy upon Cisplatin treatment................................................. 76

Fig. 19: The specific RNAPI inhibitor CX-5461 decreases H2Bub1 level. ............... 77

Fig. 20: JNK inhibition interferes with Actinomycin D-inducible H2Bub1

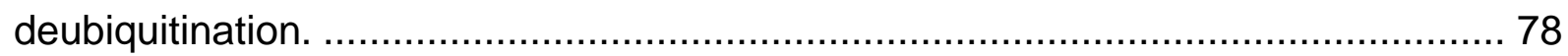

Fig. 21: AICAR treatment but not Metformin leads to reduced H2Bub1 levels. ........ 79

Fig. 22: LKB1 and AMPK depletion don`t affect the H2Bub1 level. ....................... 80

Fig. 23: AICAR treatment leads to reduced RNF20 and RNF40 target gene

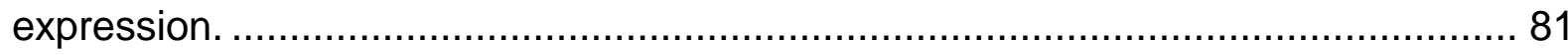


Fig. 24: H2Bub1 levels decrease upon AICAR treatment

Fig. 25: The glucose metabolism is involved in the regulation of the H2Bub1 level.. 84

Fig. 26: An H2B deubiquitinating activity is increased during cellular stress. 86

Fig. 27: USP22 regulates the level of H2Bub1. 88

Fig. 28: USP22 is needed for Cisplatin-mediated reduction of H2Bub1. 89

Fig. 29: RNF20 and RNF40 target gene expression upon Cisplatin treatment and USP22 knockdown. 90

Fig. 30: Regulation of RNF20 and RNF40 target gene expression correlates with H2Bub1 occupancy upon Cisplatin treatment and USP22 knockdown. 91

Fig. 31: Cisplatin treatment affects $\mathrm{H} 2 \mathrm{~A}$ ubiquitination. 92

Fig. 32: Knockdown of SAGA DUBm subunits reverses stress-induced loss of H2Bub1 levels.

Fig. 33: DUB module components are needed for AICAR-mediated rescue of H2Bub1 94

Fig. 34: Correlation of RNF20 and RNF40 target gene expression and H2Bub1 occupancy upon AICAR treatment and ATXN7L3 knockdown. 95

Fig. 35: RNAPII recruitment upon AICAR treatment and ATXN7L3 knockdown 96

Fig. 36: ChIP-seq profile of different histone marks. 100

Fig. 37: Average ChIP enrichment signal around TSS, in gene body and around TTS of genes. 102 


\section{Summary}

Posttranslational histone modifications are recognized as important regulators of gene expression. One of them, the monoubiquitination of lysine 120 of histone H2B (H2Bub1), has diverse functions and is involved in gene transcription as well as in controlling mRNA processing, DNA repair and DNA replication. Notably, H2Bub1 is associated with actively transcribed genes and has been linked to ongoing transcription. The misregulation of H2Bub1-modifying machinery has been observed to be tightly linked with different types of cancer. In the course of this study we clarified aspects of the regulation of the H2B ubiquitinating enzymes and presented data which support the hypothesis that the RNF20/RNF40 complex has a tumorsuppressor function probably through its regulation of H2Bub1. Moreover, we present data that demonstrate a rapid and massive loss of H2Bub1 following various cell stresses. Elucidating the mechanism and signaling pathways which control this process was one major goal of this study. It was demonstrated that H2Bub1 depletion could be rescued through knockdown of various subunits of the deubiquitinating module of the SAGA complex. ChIP-seq analyses obtained in this study demonstrated that H2Bub1 does not cover the entire transcribed region uniformly nor do the average ChIP signal profiles of H2Bub1 correlate with gene expression. However, the deubiquitination and therefore probably the new ubiquitination do correlate with gene activity.

Collectively, these studies have uncovered important mechanisms regulating H2Bub1 and may serve as a basis for developing a more solid knowledge of stress-induced $\mathrm{H} 2 \mathrm{~B}$ deubiquitination and the role of $\mathrm{H} 2 \mathrm{~B}$ monoubiquitination during tumorigenesis and metastasis. The data provide a potential role of H2Bub1 in epigenetic-based therapy for cancer. 


\section{Introduction}

\subsection{Chromatin structure}

Eukaryotic organisms have established ways of packaging DNA into chromatin since $2 \mathrm{~m}$ of genomic DNA needs to fit into a nucleus of just $10 \mu \mathrm{m}$ diameter and be accessible at the same time (Felsenfeld and Groudine, 2003).

Chromatin is a complex of DNA, histones and other proteins that form the chromosome. The basic building block of chromatin is the nucleosome which consists of DNA and histones. The typical nucleosome contains an octamer of histones with two copies of histone $\mathrm{H} 3 / \mathrm{H} 4$ heterodimers which form a stable tetramer as well as two flanking copies of histones H2A/H2B heterodimers (Kornberg, 1974). Around the histone octamer 147 base pairs of DNA are wrapped (Luger et al., 1997). The $\mathrm{H} 1$ histone is not part of the core nucleosome, but binds to linker DNA (10-80 bp long, depending on species and tissue) located between adjacent nucleosome which promotes in concert with other non-histone proteins the formation of the higher-order chromatin structures (Allan et al., 1981; Thomas, 1999).

Chromatin is compacted into several higher order structures starting with the formation of the linear $11 \mathrm{~nm}$ fiber-like structure in which nucleosomes are arranged in a beads on a string fashion separated by linker DNA (Fig. 1) (Kornberg, 1974). To increase the condensation, chromatin is packed into a thicker fiber with $30 \mathrm{~nm}$ diameter (Marsden and Laemmli, 1979). The $30 \mathrm{~nm}$ fiber produces a net compaction of roughly 50 -fold. The in vivo existence of the $30 \mathrm{~nm}$ fiber has been put into question and little is known about how these fibers are further packed within the nucleus to form the highest-order structures (Felsenfeld and Groudine, 2003).

Formally, cellular chromatin can be divided into two major states: heterochromatin and euchromatin. Heterochromatin is by definition highly condensed, contains only few genes, and is mostly transcriptionally inactive. In contrast, euchromatin contains a high density of genes and can either be actively transcribed or repressed (Grewal and Elgin, 2007; Henikoff, 2000; Richards and Elgin, 2002). However, based on recent more sophisticated methods interphase chromatin can also be categorized 
into more different types of chromatin by structure, composition, epigenetic make-up and function (Bickmore and van Steensel, 2013).

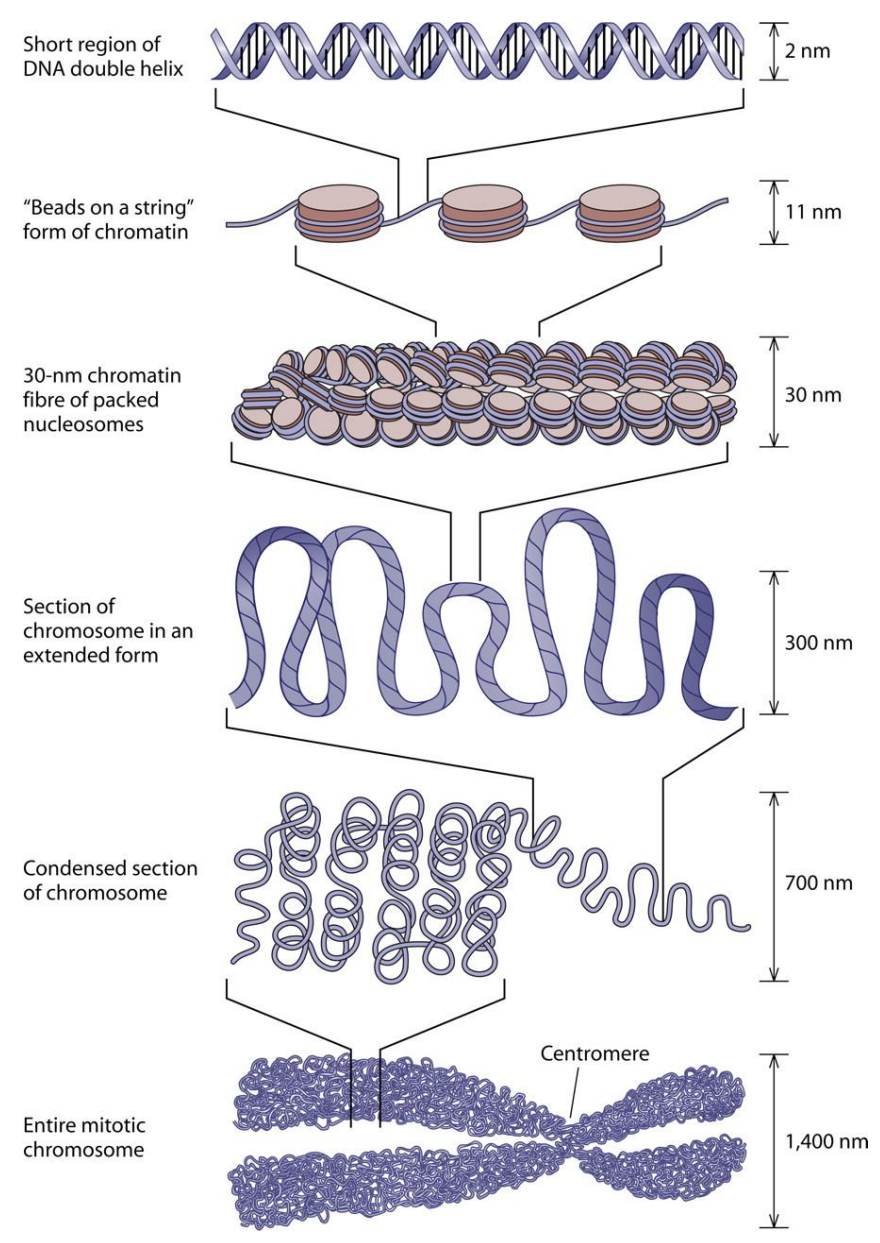

Fig. 1: Hierarchy of chromatin structure. DNA is wrapped around a histone octane to form a nucleosome. Nucleosomes connected by stretches of linker DNA in the least condensed structures are folded into a fiber-like structure of about $11 \mathrm{~nm}$ in diameter and when further compacted form a fiber with a diameter of about $30 \mathrm{~nm}$. The $30-\mathrm{nm}$ fibers can be further compacted into higher-order structures (taken from Alberts et al., 2002).

\subsection{Histone modifications}

In the cell nucleus, chromatin exists in many configurations and undergoes dynamic structural changes that play key roles in genome templated processes. The functional state of chromatin is partially regulated through posttranslational modifications (PTMs) of histones which regulate chromatin states and thus influence proper 
transcriptional regulation, nuclear architecture, cell differentiation and development (Jenuwein and Allis, 2001).

Histone proteins consist of a core domain and $\mathrm{C}$ - and N-terminal tails. The globular domain is formed by three helices and is required for the nucleosome formation (Luger et al., 1997). The so-called 'histone tails' protrude away from the DNA and thus remain at the surface of the nucleosome. This makes them accessible for enzymes and allows them to go undergo different PTMs (Jenuwein and Allis, 2001; Luger et al., 1997; Rando and Winston, 2012). The flexible positively charged Ntermini were found to mediate internucleosomal contacts (Luger et al., 1997) and can change their interaction when the chromatin fiber undergoes folding or compaction (Wolffe and Hayes, 1999). Numerous types of histone modifications exist (Fig. 2). The most studied among them are phosphorylation of serine and threonine residues, methylation of lysine and arginine and acetylation of lysine. Poorer understood modifications include ubiquitination and sumoylation of lysine and ADP-ribosylation of glutamic acid, where larger peptides or entire proteins are added (Fischle et al., 2003; Imhof, 2003; Johnsen, 2012a; Osley, 2004). The histone posttranslational modifications can influence chromatin in different manners and are therefore recognized as important regulators of protein function and stability and proteinprotein interactions (Yang, 2005). 

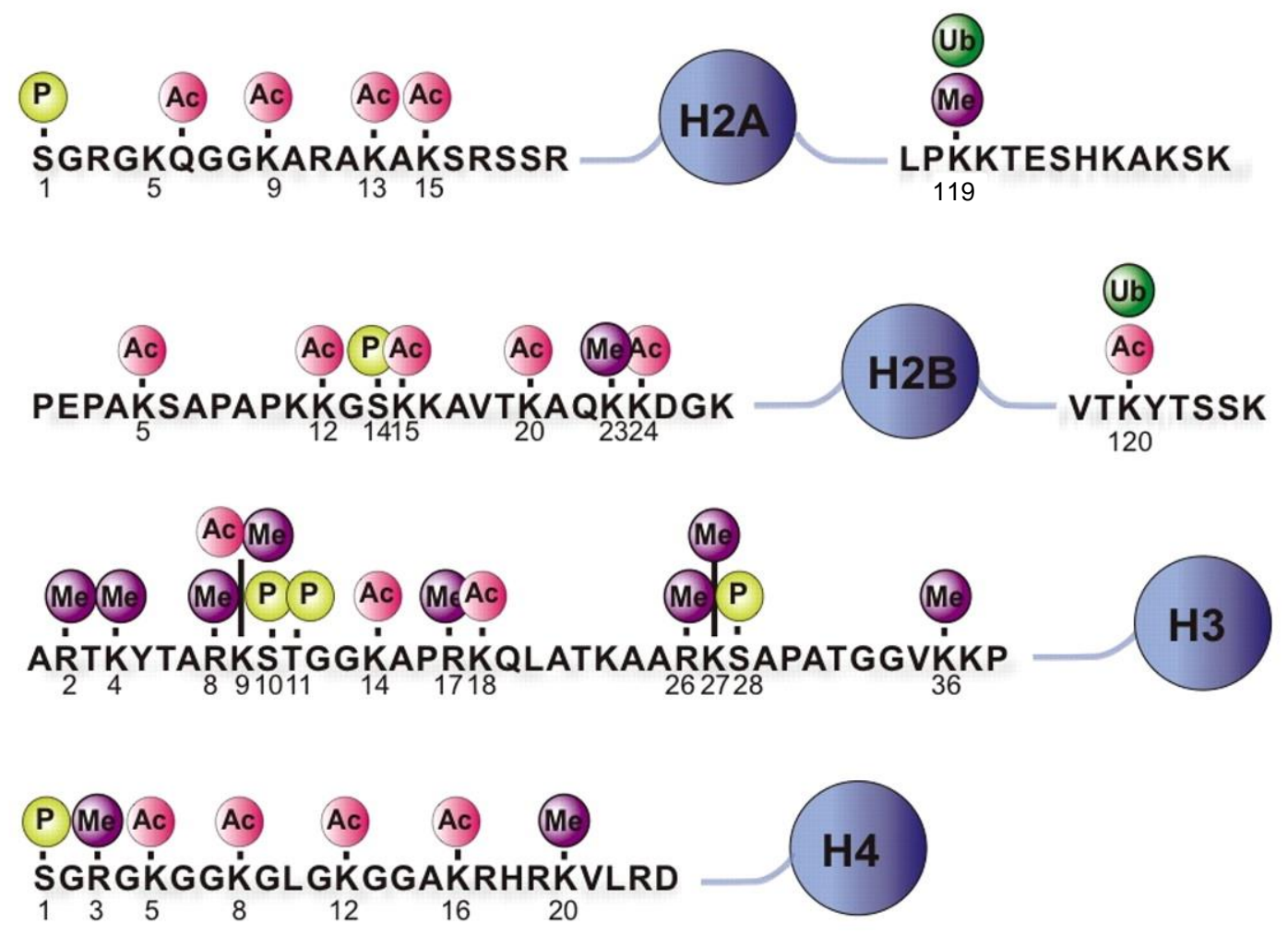

\section{Ac) Acetylation Methylation P Phosphorylation (Ub) Ubiquitination}

Fig. 2: Main posttranslational histone modifications. Histone $\mathrm{N}$-terminal tails are posttranslationally modified and certain combinations of histone modifications appear to generate a 'histone code' defining the chromatin state. The number under each amino acid indicates its position in the sequence of histones in human cells (modified from Kato et al., 2010).

\subsection{1 "Histone code"}

The high diversity of histone modifications, as well as high number of residues that can be modified and the correlation of individual modifications with various nuclear processes led to the hypothesis that specific combinations of histone modifications provide a 'histone code' (Strahl and Allis, 2000; Turner, 1993, 2000). Histone modifications can influence each other in a synergistic or antagonistic way leading to specific imprint patterns (Jenuwein and Allis, 2001). Some histone modifications are associated with transcriptional activation, others with transcriptional repression/silencing. For example, histone acetylation and deacetylation is studied in much detail and the status of this PTM is regulated enzymatically by histone acetyltransferases (HATs) and histone deacetylases (HDACs) (Strahl and Allis, 2000). Modifications within one histone can influence those of other histones in a so- 
called 'trans-histone' regulatory pathway (Fischle et al., 2003). However, the biological outputs of the histone code hypothesis are more difficult to interpret than initially hypothesized. To predict the outcomes of histone modifications much more knowledge about the so-called epigenetic writers, readers, erasers and associated non-histone proteins which affect cellular function is needed. In the context of tumorigenesis it is hypothesized that alteration in the balance between epigenetic 'on' versus 'off' chromatin states lead to inappropriate expression or silencing of gene programs (Chi et al., 2010).

\subsubsection{Misregulation of histone modifications}

Epigenetic imbalances, caused by deregulation of factors that mediate the deposition or removal of histone modifications are associated with the initiation, progression and metastasis of human cancer (Chi et al., 2010).

Histone $\mathrm{H} 3$ can be methylated at different sites and these modification can exist in different combinations which exhibits distinct distribution patterns resulting in different functional consequences for the corresponding genes in the mammalian chromosome (Barski et al., 2007). For instance, H3K4 trimethylation (H3K4me3) is strongly connected to transcriptional activation, whereas H3K27 trimethylation (H3K27me3) is frequently associated with gene silencing (Barski et al., 2007; Bernstein et al., 2006a; Mikkelsen et al., 2007). Distribution patterns of for example H3K4 and H3K27 histone modifications underlie the variety of cellular states for pluripotency and lineage differentiation. Active and repressive histone modifications can co-exist and this bivalent chromatin state provide a mechanism to maintain chromatin plasticity and is important for early stages of embryogenesis and development (Bernstein et al., 2006b; Mikkelsen et al., 2007). Epigenetic aberrations may be involved in tumor development since several reports suggest that the global loss of trimethylated $\mathrm{H} 3$ and $\mathrm{H} 4$ is a hallmark of tumor cells (Fraga et al., 2005).

Several reports demonstrated that both overexpression and inactivation of the H3K79 methyltransferase Dot1 lead to a loss of telomeric silencing (van Leeuwen et al., 2002; Ng et al., 2002a; Park et al., 2010). Dot1-dependent H3K79 methylation has also been shown to be associated with restricting the Sir proteins at heterochromatic regions (van Leeuwen et al., 2002; Ng et al., 2002a, 2003) and controlling the meiotic 
checkpoint (San-Segundo and Roeder, 2000) and DNA damage checkpoint of Rad9 in yeast (Wysocki et al., 2005). Further, misregulation of H3K79 methylation contributes to leukemogenesis in humans (Okada et al., 2005). DOT1L and H3K79 methylation are associated with transcriptional activation (Steger et al., 2008), thus providing a possible explanation for aberrant transcriptional activation found in many MLL gene fusion induced leukemia (Chi et al., 2010).

Analysis of expression of the enzyme EZH2 (Enhancer of zeste homolog 2), a H3K27-specific methyltransferase provided another connection between misregulated histone methylation marks and oncogenesis. EZH2 is frequently overexpressed in several solid tumors including prostate, breast, colon, skin and lung cancers (Bracken and Helin, 2009; Simon and Lange, 2008). RNA interferencemediated suppression of EZH2 decreases tumor growth in breast and prostate tumor xenograft models (Gonzalez et al., 2009; Yu et al., 2007). In addition to its wellknown function in epigenetic gene silencing, EZH2 is implicated in the regulation of cell differentiation in several tissue-specific stem cells (Chen et al., 2012). H3K27 diand tri-methylation are specific PcG (Polycomb group) targets (Schwartz and Pirrotta, 2008). Both PRC1 (Polycomb repressive complex 1) and PCR2 catalyze repressive histone posttranslational modifications. While PRC1 catalyzes the monoubiquitination of histone $\mathrm{H} 2 \mathrm{~A}$ at lysine 119 , PRC2 is involved in the methylation of H3K27 (Margueron and Reinberg, 2011) via its histone methyltransferase EZH2 (Neri et al., 2012). Silencing of developmental regulator genes by the two major PRCs can influence pluripotency of stem cells (Richly et al., 2010; Schwartz and Pirrotta, 2008).

\subsubsection{Histone monoubiquitination}

Ubiquitination of histone molecules was found for histones H2A, H2B, H3, H4, H2A.Z, macroH2A and $\mathrm{H} 1$. In most cases only a single ubiquitin molecule is attached to histones, which is not sufficient for targeting via the $26 \mathrm{~S}$ proteasome (Kinyamu et al., 2005; Osley, 2006; Zhang, 2003).

The first-reported ubiquitination substrate was histone H2A (Goldknopf and Busch, 1977). It has been shown that H2Aub1 is enriched in gene loci with low transcription activity and is carried out by PRC1. In mammalian cells the PRC1 complex contains two ring finger proteins, RING1A and RING1B (Cao et al., 2005; Wang et al., 2004). 
RING1B mediates H2Aub1 and was identified to bind PRC2 in the absence of PRC1 (Ku et al., 2008). Several studies demonstrated the requirement of PRC1 for stabile silencing of the gene and additional compaction (Eskeland et al., 2010; Francis et al., 2004; Stock et al., 2007). H2Aub1 is associated with the repression of developmental genes such as the Hox gene cluster (Osley, 2006). In addition, H2Aub1 not only plays a role in transcription, it has been shown to be involved in DNA repair also (Bergink et al., 2006; Dérijard et al., 1995; Doil et al., 2009; Kolas et al., 2007; Mailand et al., 2007; Marteijn et al., 2009).

\subsubsection{Histone H2Bub1}

Monoubiquitination of histone $\mathrm{H} 2 \mathrm{~B}$ (H2Bub1) is widely distributed within eukaryotes but is less abundant (1-2\% of total H2B) in contrast to H2Aub1 (Osley, 2006; Zhang, 2003). The ubiquitination of $\mathrm{H} 2 \mathrm{~B}$ occurs in a three step enzymatic process (Hochstrasser, 1996). First of all, ubiquitin gets activated by an ubiquitin activating enzyme (E1). Then, it is conjugated to a cysteine residue of an ubiquitin conjugating enzyme (E2) and finally transferred to a target lysine residue via an ubiquitin-ligase (E3) (Weake and Workman, 2008). Monoubiquitination is involved in gene transcription, DNA repair and DNA replication whereas polyubiquitination supports degradation (Sun and Chen, 2004). Polyubiquitination chains are formed by conjugating ubiquitin residues and the emerging linear polyubiquitination chains can be linked by amide bonds and thus, a number of different polyubiquitination linkages exist in cells (Kirisako et al., 2006). Different linkages seem to have distinct functions. For example, while polyubiquitination linked through $\mathrm{K} 48$ targets signals for degradation via the 26S proteasome (Glickman and Ciechanover, 2002), polyubiquitination linked through $\mathrm{K} 63$ recruits different binding partners and is involved in kinase activation (Deng et al., 2000), protein synthesis (Spence et al., 2000), DNA repair (Hoege et al., 2002; Hofmann and Pickart, 1999) and chromosome segregation (Vong et al., 2005).

In humans H2Bub1 was initially found preferentially in the transcribed region of highly expressed genes and at promoter regions of some genes (Minsky et al., 2008). In yeast H2Bub1 is required for the re-assembly of nucleosomes during elongation (Chandrasekharan et al., 2009; Xiao et al., 2005a). Therefore in both human and 
yeast, H2Bub1 was suggested to be linked to transcriptional elongation (Fleming et al., 2008; Minsky et al., 2008).

Ubiquitination of $\mathrm{H} 2 \mathrm{~B}$ was found at $\mathrm{K} 123$ in yeast and at $\mathrm{K} 120$ in other eukaryotes. The E2 enzyme for H2Bub1 in yeast was shown to be radiation-sensitive mutant protein 6 (Rad6) and the interacting E3 ligase was identified to be the RING finger protein called brefeldin A sensitive protein 1 (Bre1) (Hwang et al., 2003; Robzyk et al., 2000; Wood et al., 2003). Based on sequence alignment two homologs for the yeast Rad6 protein have been found in humans called UBE2A and UBE2B (Koken et al., 1991). A recent study could clearly show that UBE2A is the cognate $E 2$ of the BRE1 complex and therefore responsible for the H2B monoubiquitination in human cells. Rad6/UBE2A/UBE2B represents a multifunctional E2 enzyme with several cellular substrates. These are specifically targeted via the interactions with different E3 ligases. The yeast ligase Bre1 is a RING-domain ubiquitin ligase which does not possess intrinsic enzymatic activity. Bre1 is an important co-factor for Rad6-mediated ubiquitination because it is required for the recruitment of Rad6 to promoters and its association with the elongating RNA Polymerase II (RNAPII) (Henry et al., 2003; Wood et al., 2003). The human orthologs of Bre1 RNF20 and RNF40, form an obligatory heterodimer in vivo and are both required for maintaining H2Bub1 levels in human cells (Chernikova et al., 2012; Kari et al., 2011; Karpiuk et al., 2012; Pirngruber et al., 2009a). The stability of each partner is dependent upon the presence of the other and depletion of either component results in a remarkable decrease in the other as well (Kim et al., 2009; Pavri et al., 2006; Zhu et al., 2005). It has been reported that RNF20, probably through H2Bub1, activates or suppresses distinct gene classes. The sum of these effects on growth promoting and growth restrictive pathways, as well as RNF20-related alterations in human cancer, suggest a key function of RNF20 as a tumor suppressor (Shema et al., 2008). Conflicting studies have left the role of RNF40 in maintaining global H2Bub1 levels and transcription unclear (Kim et al., 2005; Weake and Workman, 2008; Zhu et al., 2005). 


\subsection{Functional role of $\mathrm{H} 2 \mathrm{~B}$ monoubiquitination}

\subsubsection{H2Bub1 and transcription}

The link between $\mathrm{H} 2 \mathrm{~B}$ monoubiquitination and transcription is complex. $\mathrm{H} 2 \mathrm{~B}$ ubiquitination has been implicated in transcriptional silencing in yeast (Briggs et al., 2002; Mutiu et al., 2007; Sun and Allis, 2002) and is suggested to have a positive role in transcriptional initiation and elongation (Henry et al., 2003; Shukla et al., 2006; Wyce et al., 2007; Xiao et al., 2005b). Gene-specific as well as genome-wide studies have revealed that $\mathrm{H} 2 \mathrm{Bub} 1$ is associated with transcriptionally active DNA and appears to occur primarily co-transcriptionally (Henry et al., 2003; Minsky et al., 2008; Tanny et al., 2007; Xiao et al., 2005a). Pavri et al (2006) performed in vitro transcription elongation assays to confirm a role for H2Bub1 in facilitating elongation by RNA polymerase II. Importantly Kim et al. (2009) showed that ongoing transcription is necessary for efficient $\mathrm{H} 2 \mathrm{~B}$ ubiquitination.

RNA polymerase II carboxyl-terminal domain (CTD) is the largest subunit of eukaryotic RNAPII that contains a conserved 52 repeated heptapeptide in humans (Egloff and Murphy, 2008). Ser5 is phosphorylated by cyclin-dependent kinase 7 (CDK7) and Ser2 is phosphorylated by cyclin-dependent kinase 9 (CDK9). While PSer5 is associated with mRNA capping and transcriptional initiation, P-Ser2 is involved in elongation, splicing and mRNA processing (Egloff and Murphy, 2008). CDK9 induces the elongation by phosphorylating negative elongation factor-E (NELF-E) and suppressor of Ty homologue 5 (SUPT5H) (Fujinaga et al., 2004; Yamada et al., 2006; Zhou et al., 2009). This hypothesis was substantiated since inhibition or depletion of CDK9 led to a global decrease in H2Bub1 levels while upon CDK9 overexpression H2Bub1 is increased (Karpiuk et al., 2012; Pirngruber et al., 2009a; Sansథ et al., 2012).

A direct connection between H2Bub1 and RNAPII CTD Ser2 phosphorylation was suggested since they have a similar pattern of localization on the active p21 gene (Gomes et al., 2006; Minsky et al., 2008). The identification of WW domaincontaining adaptor with coiled-coil protein (WAC) as part of the RNF20/RNF40 complex further supported these findings (Zhang and $\mathrm{Yu}, 2011$ ). WAC interacts 
directly with phosphorylated Ser2 and recruits the RNF20/RNF40 complex to the chromatin enabling H2B monoubiquitination (Fig. 3).

These observations show H2Bub1 as a histone mark that is coupled to RNAPII transcriptional elongation in both yeast and humans.

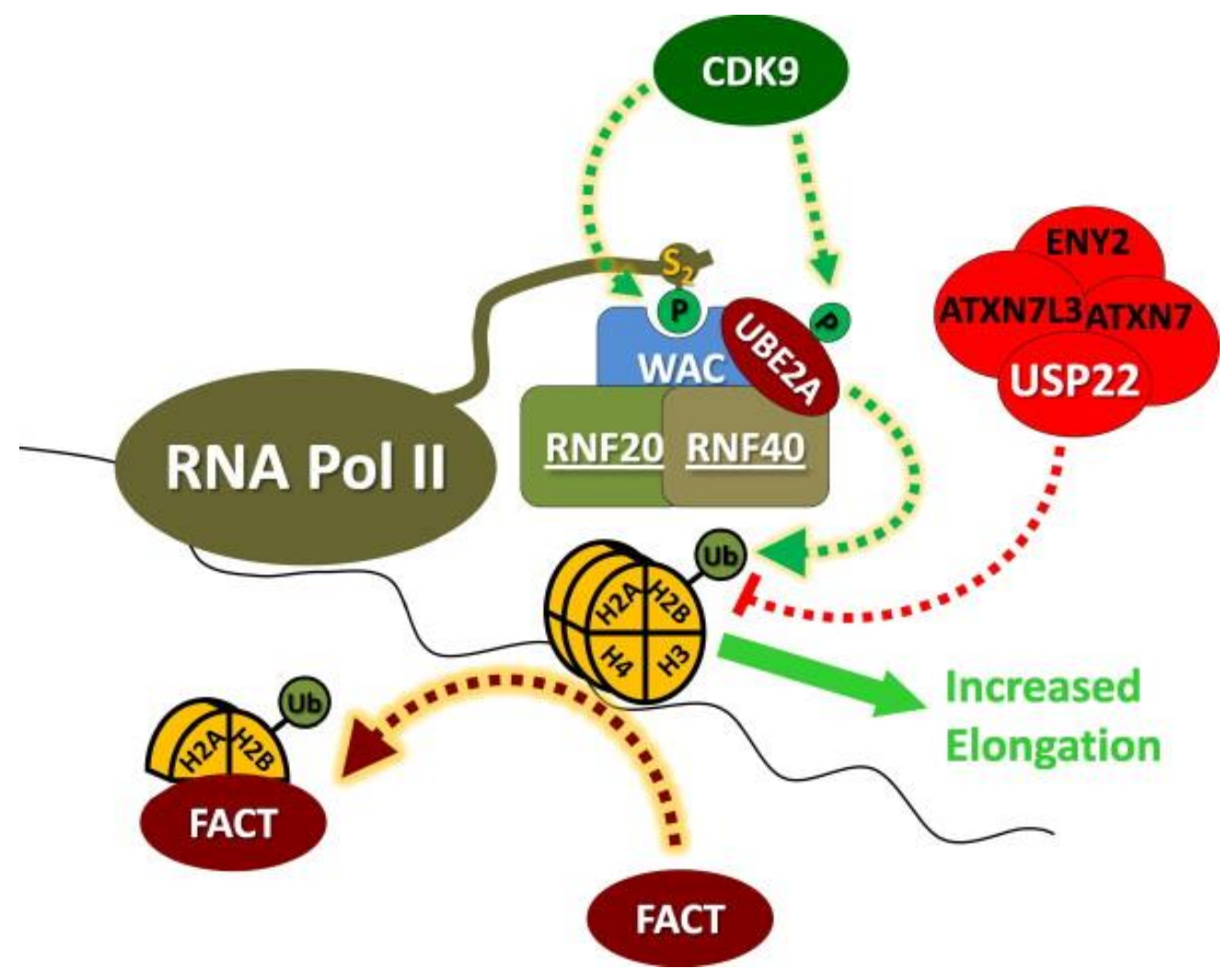

Fig. 3: H2Bub1 regulation via the CDK9-WAC-RNF20/RNF40 axis. (from Johnsen, 2012). CDK9 phosphorylates Ser2 of RNAPII CTD. WAC acts as an adaptor protein, binds to P-Ser2 and promotes recruitment of the RNF20/RNF40 complex and UBE2A to the activated gene. UBE2A is phosphorylated by CDK9 and becomes activated. Monoubiquitinated H2B leads to promotion of elongation and is then removed either by active deubiquitination via USP22 or by histone exchange via FACT complex.

\subsubsection{Cellular functions of the H2Bub1 in yeast}

In yeast H2Bub1 is excluded from heterochromatin and mostly associated with actively transcribed genes (Kao et al., 2004). H2B is transiently ubiquitinated at the coding region of several highly expressed yeast genes mediated by the activities of the ubiquitin-conjugating enzyme Rad6 and the ubiquitin ligase Bre1. Both are recruited to elongating RNA Polymerase II via yeast Paf1 (RNAPII associated factor) 
complex (Henry et al., 2003; Hwang et al., 2003; Kao et al., 2004; Robzyk et al., 2000; Wood et al., 2003; Xiao et al., 2005b). Interestingly in the absence of H2B deubiquitination, transcription of several inducible genes in yeast is reduced suggesting that H2Bub1 has to be dynamically regulated (Henry et al., 2003).

As already mentioned Paf1 is associated with RNAPII as well as with histone methyltransferases and recruits the H2Bub1 machinery to the chromatin in yeast ( $\mathrm{Li}$ et al., 2003; Shi et al., 1996; Wood et al., 2003). The Paf1 interaction with FACT (facilitates chromatin transcription) histone chaperone complex (Laribee et al., 2007) facilitates an increased $\mathrm{H} 2 \mathrm{~A} / \mathrm{H} 2 \mathrm{~B}$ heterodimer removal from the core nucleosomes. This leads to a more accessible DNA template and hence more efficient transcriptional elongation by RNAPII (Belotserkovskaya et al., 2003; Kireeva et al., 2002).

Another way by which H2Bub1 facilitates transcription is through interplay with other histone modifications. Methylation of lysine 4 in the amino-terminal tail of histone $\mathrm{H} 3$ is mediated by COMPASS1 (complex of proteins associated with Set1), a multiprotein complex (Briggs et al., 2001; Krogan et al., 2002; Miller et al., 2001; Nagy et al., 2002; Roguev et al., 2001) which is required for telomeric silencing of gene expression (Briggs et al., 2001; Krogan et al., 2002). The ubiquitin-conjugating enzyme Rad6 is required for methylation of lysine 4 of histone H3 (H3K4me) since H2Bub1 serves as recognition signal for Set1/COMPASS recruitment (Dover et al., 2002).

In addition to $\mathrm{H} 3 \mathrm{~K} 4$ methylation, H2Bub1 promotes $\mathrm{H} 3 \mathrm{~K} 79$ methylation through a socalled trans-tail mechanism (Briggs et al., 2002; Dover et al., 2002; $\mathrm{Ng}$ et al., 2002b; Sun and Allis, 2002). H2Bub1 has been shown to recruit the histone methyltransferases Set1 and Dot1 to di- and trimethylate H3K4 and H3K79 and facilitate histone cross talk in yeast and mammals (Fierz et al., 2011; Kim et al., 2009; Lee et al., 2007; Sun and Allis, 2002).

\subsubsection{The role of H2Bub1 in higher eukaryotes}

While the role of H2Bub1 in yeast is well explored, the precise functional role in mammalian cells remains largely unknown. H2Bub1 depletion alters the expression of a subset of genes in mammalian cells. The fact that H2Bub1 is required for 
repression of certain genes suggests that this modification plays a complex role in mammalian cellular processes (Shema et al., 2008).

Whereas H2Bub1 and $\mathrm{H} 3 \mathrm{~K} 36 \mathrm{me} 3$ in mammalian cells are associated with transcribed regions of active genes, $\mathrm{H} 3 \mathrm{~K} 4 \mathrm{me} 3$ was preferentially found at the transcriptional start sites and $5^{\prime}$ ends of genes (Minsky et al., 2008). Similarly to $S$. cerevisiae, H2Bub1 in higher eukaryotes facilitates efficient transcriptional elongation through the FACT complex (Pavri et al., 2006; Schwabish and Struhl, 2004). Conversely, H2Bub1 could modulate chromatin dynamics by promoting FACT function during transcriptional elongation (Fleming et al., 2008; Pavri et al., 2006). Structural studies previously showed that H2Bub1 facilitates cancer by providing a more 'open' chromatin conformation (Fierz et al., 2011). Moreover, the addition of a similar, but chemically distinct moiety with different surface charges did not mimic the action of H2Bub1. This was shown in yeast, where sumoylation did not have the same effects compared to H2Bub1 on transcription (Batta et al., 2011) Thus, the decompaction of the chromatin seems to be dependent on the chemical nature of ubiquitin (Fierz et al., 2011).

Furthermore, an additional role for H2Bub1 was shown in the regulation of gene expression by interfering with 3'end mRNA processing. Pirngruber et al. (2009) showed the requirement of H2Bub1 for correct stem loop-dependent processing of histone genes and revealed a new role for CTD phosphorylation in controlling this process since $\mathrm{H} 2 \mathrm{Bub} 1$ regulation is mediated by CDK9 via a CTD-dependent PAFRNF20/RNF40 complex (Pirngruber et al., 2009a).

Apart from its role in transcription, a substantial amount of evidence has accumulated that H2Bub1 also participates in other cellular processes including DNA damage signaling and cell cycle checkpoint activation (Chernikova et al., 2012; Kari et al., 2011; Moyal et al., 2011; Nakamura et al., 2011). The E3 ubiquitin ligases RNF20 (Matsuoka et al., 2007; Mu et al., 2007) and RNF40 (Mu et al., 2007; Stokes et al., 2007) were identified as substrates of the ATM and ATR kinases. It is proposed that their recruitment upon double strand break (DSB) generation to the DSB-site is required for recruitment of the repair machinery (Moyal et al., 2011). A mechanistic role for RNF20 and RNF40 in DNA double-strand break repair remained unknown 
although studies provide a role for H2Bub1 in the process of homologous recombination and non-homologous end joining DNA repair (Moyal et al., 2011; Nakamura et al., 2011). An implication of FACT in the exchange of $\mathrm{YH} 2 \mathrm{AX} / \mathrm{H} 2 \mathrm{~B}$ dimers was shown in a recent study (Heo et al., 2008) and in addition our group proposed the importance of RNF40 activity for proper FACT recruitment to chromatin upon DNA damage (Kari et al., 2011).

\subsubsection{Genome-wide distribution of H2Bub1}

In a previous chapter it was mentioned that the dynamic turnover of H2Bub1 is critical to both transcription and gene silencing. H2Bub1 regulates chromatin dynamics by enhancing nucleosome stability (Chandrasekharan et al., 2009) and disrupting local and higher-order chromatin compaction (Fierz et al., 2011). Out of these findings H2Bub1 seems to be involved in additional regulatory pathways besides transcrosstalk with the methylations of histone $\mathrm{H} 3$. To investigate genome-wide properties and unique roles of H2Bub1 in cooperation with other histone modifications Minsky et al. (2008) generated a highly specific H2Bub1 antibody and performed ChIP-chip and ChIP-seq analysis.

Recently published high-throughput sequencing (ChIP-seq) data for the genomewide occupancies of H2Bub1 in human teratocarcinoma NCCIT cells showed that H2Bub1 is a 5'-enriched active transcription mark of almost all expressed genes with its levels progressively decreasing towards the 3' region (Jung et al., 2012). Furthermore, H2Bub1 was strongly enriched at the exon-intron boundaries of highly expressed exons and in contrast $\mathrm{H} 2 \mathrm{Bub} 1$ was depleted from non-expressed genes and from intergenic regions. H2Bub1 distribution correlates with that of $\mathrm{H} 3 \mathrm{~K} 36 \mathrm{me} 3$ and H3K79me2, two marks associated with transcriptional elongation (Jung et al., 2012). Similar observations were made in Drosophila melanogaster or Arabidopsis thaliana using the same antibody that recognizes H2Bub1 in these species (Kharchenko et al., 2011; Roudier et al., 2011; Roy et al., 2010). This distribution profile is in agreement with the known interaction of RAD6/RNF20/RNF40 with the PAF elongation complex that is recruited by the elongation form of RNAPII (Laribee et al., 2007). 


\subsubsection{H2Bub1 and its regulation in human cancer}

The first evidence of an epigenetic link of monoubiquitinated $\mathrm{H} 2 \mathrm{~B}$ to cancer was shown by several reports that demonstrated the loss of H2Bub1 during carcinogenesis (Hahn et al., 2012; Prenzel et al., 2011; Shema et al., 2008; Urasaki et al., 2012).

The first indication of a potential tumor suppressor role of RNF20 came from Shema et al. (2008) which demonstrated DNA hypermethylation of the RNF20 promoter in breast cancers. Further evidence was provided by the demonstration of oncogenic cell characteristics, like enhanced cell migration, growth in soft agar, and the ability of cells to form tumors in xenograft models. All these assays showed a higher oncogenic potential of RNF20-depleted cells. A strong decrease in expression of the tumor suppressor p53 upon RNF20-knockdown supports these results (Shema et al., 2008). A previous study showed that RNF20 can stimulate p53-dependent transcription via its capability to bind p53 (Kim et al., 2005). Taken together, the results indicate that RNF20 has a tumor suppressor role but the role of RNF40 in tumor suppression remains largely unknown. Recently our group showed that siRNAmediated knockdown of the H2B ubiquitin ligase RNF40 decreases ER $\alpha$-induced gene transcription and supports estrogen-independent cell proliferation and activation of certain cell survival signaling pathways (Prenzel et al., 2011). Furthermore, a nearly complete absence of H2Bub1 was found in malignant and metastatic breast cancer tissues whereas strong signals for H2Bub1 expression was detected in adjacent non-transformed mammary epithelium, arguing for a decrease in H2Bub1 expression concomitantly with tumor progression (Prenzel et al., 2011). Taken together these observations support a potential tumor suppressor role for $\mathrm{H} 2 \mathrm{~B}$ monoubiquitination and its ubiquitin ligases RNF20 and RNF40.

\subsection{Histone H2B deubiquitination}

H2B monoubiquitination is highly dynamic and its level is not only regulated by addition, but also by active removal of the ubiquitin residue from the chromatin. The dynamic nature of H2Bub1 was revealed by the identification of specific 
deubiquitinases in different organisms. Ubp8, a subunit of the transcriptional coactivator Spt-Ada-Gcn5-Acetylating complex (SAGA) which plays a role in regulating gene expression, was the first identified H2B deubiquitinase in S. cerevisiae (Daniel et al., 2004; Henry et al., 2003). Another ubiquitin protease, Ubp10 was shown to deubiquitinate $\mathrm{H} 2 \mathrm{~B}$ in $\mathrm{S}$. cerevisiae independent of SAGA at distinct target loci. While Ubp8 functions at promoters of certain SAGA-dependent genes, Ubp10 is associated with non-transcribed regions and regulates silencing of telomeres, rDNA and other loci through histone H2B deubiquitination (Daniel et al., 2004; Emre et al., 2005; Gardner et al., 2005). The steady state levels of H2Bub1 were increased in ubp8 or in $u b p 10$ cells but were further increased in a double ubp 8 ubp 10 mutant suggesting that these two deubiquitinases target different loci in the yeast genome (Emre et al., 2005; Gardner et al., 2005). Buszczak et al. (2009) described the Drosophila gene scrawny, which regulates multiple types of stem cells. Scrawny is a USP family protein and deubiquitinates H2B. It shares homology with yeast Ubp10 within the core protease domain and closely matches human USP36 (Buszczak et al., 2009). In yeast the deubiquitinating enzyme Ubp8 is recruited to the gal1 promoter and the transcription of SAGA-responsive genes can be decreased upon its deletion (Kohler et al., 2006a). Furthermore, Ubp8 is known as part of the SLIK (SAGA-like) complex, where it catalyzes the differentially regulation of $\mathrm{H} 3$ methylation at some SAGA promoters (Lee et al., 2005). In isolation from other proteins Ubp8 is inactive (Lee et al., 2005) and is unable to bind to free ubiquitin (Ingvarsdottir et al., 2005). Thus, it was speculated that different binding partners induce conformational changes which regulate Ubp8 activity (Bonnet et al., 2008).

\subsubsection{The SAGA complex}

The yeast SAGA complex consists of 21 widely conserved proteins. The complex unites acetylating and deubiquitinating enzymatic activities. The main component of the acetylating complex is GCN5 - a bromodomain-containing protein that mediates acetylation of H3 (Grant et al., 1997). GCN5 was originally identified as a transcriptional co-activator in yeast and enhances transcription through its intrinsic acetyltransferase activity which facilitates acetylation of histones and non-histone substrates (Brownell et al., 1996; Sterner and Berger, 2000). 
The SAGA deubiquitination module (DUBm) is composed of Sgf11, Sus1 and Ubp8 (Kohler et al., 2006b) with corresponding human orthologs ATXN7L3, ENY2 and USP22 (Fig. 4). The deubiquitination activity of the SAGA complex is highly conserved as revealed by the identification of Ubp8 orthologs in S. pombe (Ubp8), $D$. melanogaster (Nonstop) and human (USP22) (Helmlinger et al., 2008; Weake et al., 2008; Zhang et al., 2008a; Zhao et al., 2008).

\subsubsection{USP22 as the main deubiquitinase for H2B}

Deubiquitination of $\mathrm{H} 2 \mathrm{~B}$ in humans is predominantly dependent on the SAGA complex and its disruption via ATXN7L3 knockdown leads to a great increase in the H2Bub1 level (Lang et al., 2011). However, the role of USP22 as the main DUB for H2B is not well established. Consistent with a potential tumor suppressor role of RNF20 and RNF40, oncogenic function is proposed for USP22 (ubiquitin-specific protease 22) that catalyzes deubiquitination, the opposite enzymatic reaction to RNF20 and RNF40. Transcriptional profiling of tumor cells generated an 11-gene signature associated with poor prognosis (Glinsky, 2005). USP22 was discovered as part of this group of genes. Two later reports described oncogenic effects of USP22 in distinct transcriptional networks (Zhang et al., 2008a; Zhao et al., 2008). The first one identified USP22 as a positive regulator of MYC-dependent transcription potentially accounting for its oncogenic properties (Zhang et al., 2008a). USP22 was shown to be a positive regulator of p53-dependent transcription and RNF20 was described as a co-activator of p53-dependent activation of p21 and MDM2 (Kim et al., 2005). These findings hypothesize that only a correct balance between RNF20 and USP22 activities may conduce to optimal p53-dependent transcriptional activation. Recently published data showed that USP22 is implied in the deubiquitination of H2B at the interferon-regulated gene IRF1 gene (Chipumuro and Henriksen, 2012) and is essential for androgen-dependent transcription (Zhao et al., 2008). Thus, overexpression of USP22 may contribute to development of prostate cancer. An implication of USP22 in breast cancer was reported too, in part based on the finding that estrogen receptor (ER)-mediated transactivation requires USP22 (Zhao et al., 2008). However, depletion of USP22 results in only a mild increase in the H2Bub1 level (Chipumuro and Henriksen, 2012; Zhang et al., 2008a; Zhao et al., 2008). Due to these facts a model of allosteric regulation of USP22 by SAGA was 
proposed, where enzymatic activity of this enzyme depends on interactions with two other subunits of the deubiquitinating module (DUBm) ATXN7 and ATXN7L3 (Rodriguez et al., 2012). In addition it was reported that despite of USP22, ATXNL3 and ENY2 are required for AR-mediated transactivation (Zhao et al., 2008).

It is also possible that other ubiquitin hydrolases deubiquitinate H2Bub1 in addition to USP22. For example, the enzyme USP27X shares structural homology with USP22 and is known to interact with it (Sowa et al., 2009) suggesting USP27X as another DUB for H2Bub1. Furthermore, a tblastn search revealed for both USP27X (31\% over 478 amino acids) and USP51 (30\% over 474 amino acids) a degree of homology to Ubp8 similar to that of USP22 (32\% identity over 462 amino acids) (Johnsen, 2012a). To determine whether USP22, USP27X, USP51 and perhaps other DUBs contribute to deubiquitination of $\mathrm{H} 2 \mathrm{~B}$ and have overlapping functions in certain tissues, additional studies will be necessary.

A
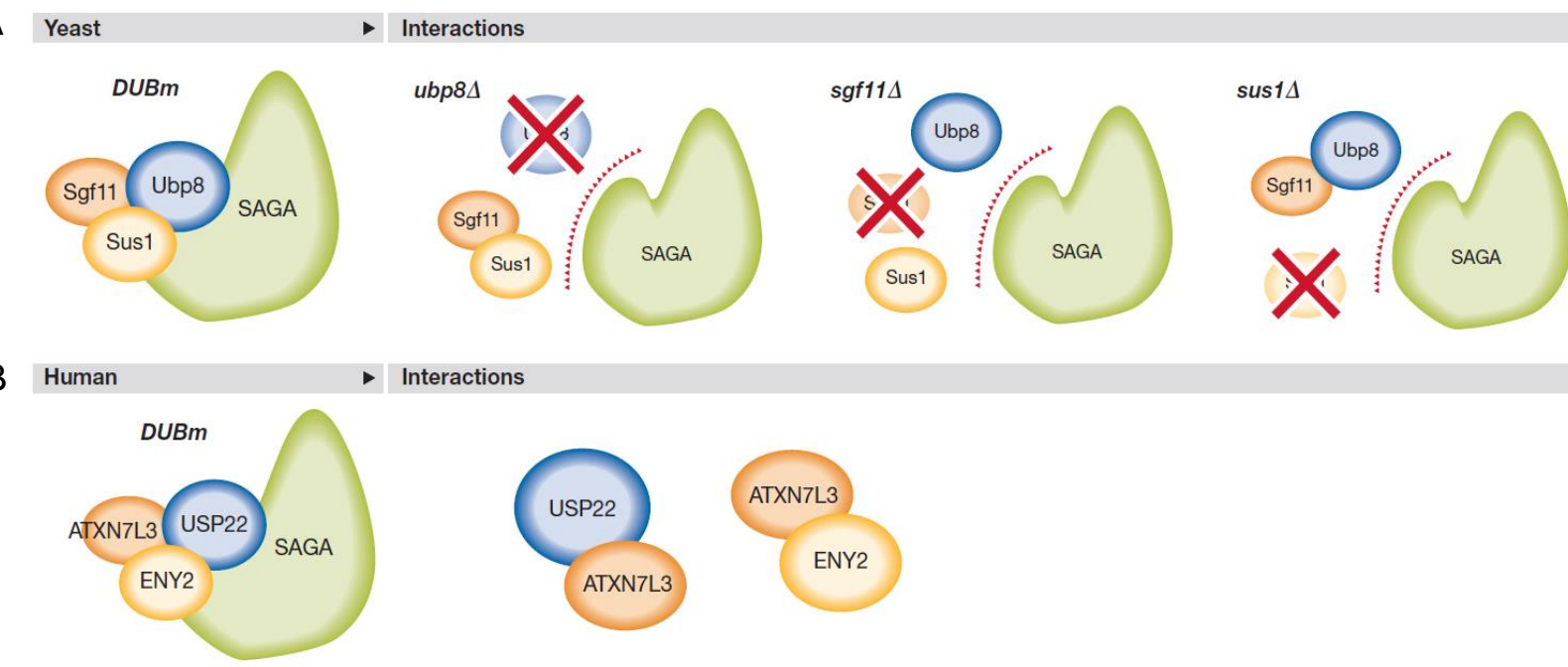

Fig. 4: The SAGA deubiquitination module is conserved from yeast to humans. (from RodriguezNavarro, 2009) (A) The yeast DUB module is composed of Ubp8, Sgf11 and Sus1. The elimination of one of the subunits induces a loss of the other members and impairs SAGA association. (B) The human DUBm contains USP22, ATXN7L3 and ENY2. A direct interaction between ATXN7L3-USP22 and ATXN7L3-ENY2 has been shown in human cells.

\subsubsection{The SAGA deubiquitination module}

The Sus1/ENY2 subunit of SAGA in yeast and humans is essential for transcription elongation (Kopytova et al., 2010a, 2010b; Pascual-García and Rodríguez-Navarro, 
2009). ENY2 interacts with TREX-2 messenger ribonucleic acid (mRNA) export complex that acts at the interface between transcription and mRNP (ribonucleoprotein) export (Pascual-García and Rodríguez-Navarro, 2009). Thus, Sus1/ENY2 has many 'satellite' partners in interactions, and all of them together coordinate transcription, mRNP biogenesis and export (Kopytova et al., 2010a; Kurshakova et al., 2007). Some SAGA complexes that are present at the nuclear periphery also interact with the nuclear core complex (Kurshakova et al., 2007). Compared to Sus1/ENY2, much less is known about the function of the Sgf11/ATXN7L3 subunit of the DUB module (Gurskiy et al., 2012). Knockdown of the ATXN7L3 subunit of the SAGA complex leads to dissociation of USP22 from the SAGA complex whereas the other SAGA activities are preserved. In these cells, the H2Bub1 levels were strikingly increased (by 3-4 fold) demonstrating that the SAGA DUB activity is a major regulator of this modification and cannot be fully compensated by other deubiquitinases (Lang et al., 2011). Another SAGA subunit is ATXN7, a protein mutated in the inherited neurodegenerative disorder spinocerebellar ataxia type 7 (SCA7) (McCullough et al., 2012).

\subsubsection{Other H2Bub1 deubiquitinases}

In addition to USP22, several other DUBs have been identified as H2B DUBs in humans. Nicassio and colleagues reported that USP3 can remove ubiquitin from H2Bub1 in vivo and described USP3 as the closest human homolog of yeast Ubp8 (Nicassio et al., 2007). Moreover, studies have described USP7 (also known as HAUSP) to be an H2B deubiquitinase in Drosophila and in human cells and functions as a major p53 regulator (van der Knaap et al., 2005, 2010). Analysis of mass spectrometry data identifies histones as interaction partners with the highest Z-scores for USP8 and USP44 (Sowa et al., 2009) and a recent report identified USP44 as an H2B deubiquitinase in ES cells (Fuchs et al., 2012). Single reports identified other H2B deubiquitinases in Drosophila or in $A$. thaliana but such activities were not reported for their human orthologs so far (Buszczak et al., 2009; Sridhar et al., 2007). Overall, it can be expected that in addition to SAGA, which appears as the major H2B deubiquitination complex in mammals, other DUBs will have specific effects in controlling H2Bub1 and H2Bub1-dependent processes. 


\subsection{Nucleoli and stress conditions}

\subsubsection{Nucleoli}

The nucleolus is one of the largest organelles in eukaryotic cells. The main function of the nucleolus is to carry out the initial steps of one of the most important cellular processes, ribosome biogenesis. This process must be tightly regulated to achieve proper cell growth and cellular proliferation (Lempiäinen and Shore, 2009). Beside the ribosome subunit biogenesis the nucleolus is involved in additional cellular functions (Pederson and Tsai, 2009), such as RNP biogenesis, cell-cycle control, apoptosis, DNA replication and DNA repair (Ahmad et al., 2009).

With the help of electron microscopy three structural distinct subnucleolar compartments could be defined, the fibrillar centers where the transcription takes place, the dense fibrillar compartment here the rRNA processing happens and the granular component where the assembly of ribosomal subunits occurs (Boisvert et al., 2007). RNA polymerase I (RNAPI) transcribes rDNA genes, leading to the synthesis of a $47 \mathrm{~S}$ precursor ribosomal rRNA transcript (pre-rRNA). The processing of pre-rRNA occurs either co- or posttranscriptionally and involves modifications like cleavage, methylation, and pseudouridylation of the primary rRNAs (Smith and Steitz, 1997; Stoykova et al., 1985). Several ribonucleoprotein complexes control the cleavage and function to remove the external transcribed spacers (5'ETS and $3^{\prime} E T S$ ) and the internal transcribed spacers (ITS1 and ITS2). To form the small and large pre-ribosome subunits the $28 \mathrm{~S}, 18 \mathrm{~S}$, and $5.8 \mathrm{~S}$ rRNAs are assembled with ribosomal proteins. Final processing steps conduct the pre-ribosome subunits to become the mature $40 S$ and 605 ribosome subunits (Boulon et al., 2010).

\subsubsection{Nucleolar reaction to stress}

All organisms need to react to conditions that influence their homeostatic balance. The cellular response to stress is an adjustment to environmental factors, such as nutrient supply, temperature or oxidative stress. Cellular stress can influence ribosome subunit production and cell growth (Mayer and Grummt, 2006). These stresses are often accompanied by drastic changes in the organization and structural constitution of the nucleolus. The induction of DNA damage and transcriptional 
inhibition causes nucleolar segregation, a well-described phenomenon (Al-Baker et al., 2004; Govoni et al., 1994). Segregation is marked by condensation followed by separation of the fibrillar centers and granular component, together with the formation of nucleolar caps from nucleolar and nuclear proteins around the nucleolar remnant (Shav-Tal et al., 2005). Stress conditions can alter the protein composition of the nucleolus very fast, indicating a complex reorganization during the stress response (Boisvert et al., 2010). Factors which are involved in nucleolar stress have been shown to translocate quite rapid so that some components change their localization already two minutes after adding the drug (Cohen et al., 2008). A typical mechanism of cell cycle regulation by nucleolar signaling involves the alteration of the p53 level. A previous report proposed a p53-dependent mechanism to redistribute ribosomal proteins from the nucleolus to the nucleoplasm (Boisvert and Lamond, 2010). In normally growing cells p53 protein levels are kept low due to its constant ubiquitination by the E3 ubiquitin ligase HDM2 which marks p53 for proteosomal degradation (Kruse and $\mathrm{Gu}, 2009$ ). Different mechanisms which regulate the p53nucleolar signaling pathways therefore can induce increased cellular p53 levels (Lee and $\mathrm{Gu}, 2010)$. Furthermore, posttranslational modifications of nucleolar proteins may also be important for protein localization upon stress-induction (Boulon et al., 2010). Importantly, a recent study described that a large variety of chemotherapeutic drugs activate ribosomal stress via inhibiting transcription and processing of ribosomal RNA (Burger et al., 2010).

\subsubsection{Nucleoli and JNK signaling}

Stress stimuli have been shown to activate c-Jun NH2-terminal protein kinase (JNK) family members. JNKs belong to the family of stress-activated protein kinases (SAPKs) and are implicated in cellular response to environmental stress and regulate the choice between survival and apoptosis (Chen et al., 1996). The activity of JNKs is induced by exposure to UV-B radiation, alkylating agents, hyperosmotic shock, proinflammatory cytokines and oxidative damage (Martindale and Holbrook, 2002; Song and Lee, 2007; Yoon and Kim, 2004). Activated JNKs phosphorylate several target proteins to change their activity and induce various biological effects. Phosphorylation of threonine and tyrosine residues in the $\mathrm{T}^{*} \mathrm{PY}^{*}$ motif of $\mathrm{JNK}$ by SEK1/MKK4 regulate their activity (Dérijard et al., 1995). JNK2 can phosphorylate 
TIF-1A, an essential transcription factor that modulates RNA polymerase I (RNAPI) activity (Mayer et al., 2005). Phosphorylated TIF-1A cannot interact with RNAPI and thus impairs the assembly of the transcription complex and rRNA synthesis. Furthermore, stress-induced inactivation of TIF-IA is associated with the translocation of TIF-IA from the nucleolus to the nucleoplasm. This shows the stress-induced release of nucleolar proteins to achieve different regulatory functions (Mayer and Grummt, 2005).

\subsubsection{AMPK signaling in tumorigenesis and tumor metabolism}

Metabolic reprogramming includes high rate of glycolysis followed by oxygenindependent lactate fermentation. This observation is known as the Warburg effect and is a hallmark of cancer cells (Hanahan and Weinberg, 2011; Hsu and Sabatini, 2008; Koppenol et al., 2011). Upon stress conditions tumor cells need this reprogramming to grow and survive (Christofk et al., 2008). AMP-activated protein kinase (AMPK) is a metabolic sensor that plays a central role in the regulation of cellular energy homeostasis. This highly conserved Ser/Thr protein kinase has been recently linked to stress resistance (Bungard et al., 2010; Jeon et al., 2012; Liu et al., 2012). Due to its involvement in cellular stress resistance, AMPK has been linked to the regulation of tumorigenesis but the precise role remains unknown (Shackelford and Shaw, 2009). 


\section{Materials}

\subsection{Technical equipment}

Agarose gel chamber

Balance

Bandelin Sonoplus Sonicator

Biological Safety Cabinet "Hera Safe"

Bioruptor

Centrifuge (Megafuge 1.OR)

Centrifuge $4^{\circ} \mathrm{C}(5417 \mathrm{R})$

C1000 ${ }^{\mathrm{TM}}$ Thermal Cycler

CFX96 ${ }^{\mathrm{TM}}$ Optical Reaction Module

Counting chamber (Neubauer)

5100 Cryo $1^{\circ} \mathrm{C}$ Freezing Container

Electrophoresis \& Electrotransfer Unit

Freezer $-20^{\circ} \mathrm{C}$

Freezer $-80^{\circ} \mathrm{C}$ "Hera freeze"

Gel Imager "Gel iX imager"

Incubator (bacteria)

Incubator (bacteria culture)

Incubator (cell culture) "Hera cell 150"

Inverse Microscope "Axiovert 40 CFL"

Luminometer 2030-100

Magnet stirrer "MR3001"

Microscope "Axiovert 40 C"

Microwave

Nano Drop® ND-1000 Spectrophotometer
Harnischmacher Labortechnik, Kassel

Sartorius AG, Göttingen

Bandelin electr. GmbH \& Co. KG, Berlin

Thermo Fisher Scientific, Waltham, USA

Thermo Fisher Scientific, Waltham, USA

Thermo Fisher Scientific, Waltham, USA

Eppendorf AG, Hamburg

Bio-Rad Laboratories $\mathrm{GmbH}$, München

Bio-Rad Laboratories GmbH, München

Brand GmbH \& Co. KG, Wertheim

Thermo Fisher Scientific

GE Healthcare Europe $\mathrm{GmbH}$, München

Liebherr $\mathrm{GmbH}$, Biberach

Thermo Fisher Scientific, Waltham, USA

Intas Science Imaging $\mathrm{GmbH}$, Göttingen

Memmert GmbH \& Co. KG, Schwabach

Infors AG, Bottmingen

Thermo Fisher Scientific, Waltham, USA

Carl Zeiss Microlmaging $\mathrm{GmbH}$, Göttingen

Turner designs, Sunnyvale, CA, USA

Heidolph GmbH \& Co. KG, Schwabach

Carl Zeiss Microlmaging $\mathrm{GmbH}$, Göttingen

Clatronic International $\mathrm{GmbH}$, Kempen

Peqlab Biotechnology $\mathrm{GmbH}$, Erlangen 
Pestle

$\mathrm{pH}$ meter inoLab®

Pipette Aid® portable XP

Pipettes "Research" Series

Power supply "Power Pack P25T"

Qubit® 2.0 Fluorometer.

Refrigerator

Repeat Pipette

ScanScope XT

Scanner (CanoScan 8600F)

Shaker "Rocky"

Table centrifuge (GMC-060)

Test tube rotator

Ultrapure Water System "Aquintus"

Vacuum pump

Vortex mixer

Water bath "TW 20"

X-Ray Cassettes

\subsection{Consumable materials}

Cellstar 6- and 12-well cell culture plate

Cellstar PP-tube 15 and $50 \mathrm{ml}$

Cellstar tissue culture dish $100 \times 20 \mathrm{~mm}$

Cellstar tissue culture dish $145 \times 20 \mathrm{~mm}$

Cell scraper $(16 \mathrm{~cm}, 25 \mathrm{~cm})$

Cryo Tube ${ }^{\mathrm{TM}}$ Vial $(1.8 \mathrm{ml})$

Falcon® assay plate, 96 well
Sartorius AG, Göttingen

WTW GmbH, Weilheim

Drummond Scientific Co., Broomall, USA

Eppendorf AG, Hamburg

Biometra GmbH, Göttingen Material 26

Invitrogen $\mathrm{GmbH}$, Karlsruhe

Liebherr $\mathrm{GmbH}$, Biberach

Gilson Inc., Middleton, USA

Aperio, Vista, CA, USA

Canon $\mathrm{GmbH}$, Krefeld

Schütt Labortechnik GmbH, Göttingen

LMS Co., Ltd., Tokyo, Japan

Schütt Labortechnik GmbH, Göttingen

membraPure $\mathrm{GmbH}$, Bodenheim

Integra Bioscienc. AG, Zizers, Switzerland

Scientific Industries, Inc., Bohemia, USA

JULABO Labortechnik GmbH, Seelbach

Rego X-ray GmbH. Augsburg

Greiner Bio-One GmbH, Frickenhausen

Greiner Bio-One GmbH, Frickenhausen

Greiner Bio-One GmbH, Frickenhausen

Greiner Bio-One GmbH, Frickenhausen

Sarstedt AG \& Co., Nümbrecht

Thermo Fisher Scientific, Waltham, USA

VWR Int., LLC, West Chester, USA 
Gel blotting paper (Whatman paper)

Glass coverslips (18 mm)

HybondTM-PVDF Transfer Membrane

Microtube 0,5 ml, $1.5 \mathrm{ml}, 2 \mathrm{ml}$

Microtube $1.5 \mathrm{ml}$, conical

96 Multiply® PCR plate white

96-well Multiplate ${ }^{\circledR}$ PCR plate white (low)

NORM-JECT Syringes of different volume

FrameStar® 96Skirted qRT-PCR plates

Parafilm® "M"

PET track-etched cell culture inserts

Petri dish $92 \times 16 \mathrm{~mm}$

Pipette tips

Pipette filter tips

Pippin Prep ${ }^{\mathrm{TM}}$ System

Protan® Nitrocellulose transfer membrane

Shandon Coverplate

Syringe filter, Ca-membrane, 0,20 $\mu \mathrm{m}$

Tissue microarrays

X-ray films "Super RX"

\subsection{Chemicals}

\subsubsection{General chemicals}

Acetic acid

Adefodur WB developing concentrate

Adefodur WB fixing concentrate

Adenosin triphosphate (ATP)
Sartorius AG, Göttingen

Gebr. Rettberg GmbH, Göttingen

GE Healthcare Europe $\mathrm{GmbH}$, München

Sarstedt AG \& Co., Nümbrecht

VWR International GmbH, Darmstadt

Sarstedt AG \& Co., Nümbrecht

Bio-Rad Laboratories GmbH, München

Henke Sass Wolf GmbH, Tuttlingen

4titute Ltd., Wotton, UK

Pechiney Plastic Packaging, Chicago, USA

BD Bioscience, Franklin Lakes, NJ, USA

Sarstedt AG \& Co., Nümbrecht

Greiner Bio-One GmbH, Frickenhausen

Sarstedt AG \& Co., Nümbrecht

Invitrogen $\mathrm{GmbH}$, Karlsruhe

Whatman $\mathrm{GmbH}$, Dassel Material 27

Thermo Fisher Scientific, Waltham, USA

Sartorius AG, Göttingen

US Biomax,Inc., Rockville, MD, USA

Fujifilm Corp., Tokyo, Japan
Carl Roth GmbH \& Co. KG, Karlsruhe

Adefo-Chemie $\mathrm{GmbH}$, Dietzenbach

Adefo-Chemie $\mathrm{GmbH}$, Dietzenbach

Fermentas $\mathrm{GmbH}$, St. Leon-Rot 
Agarose

Albumin Fraction $\mathrm{V}$ (BSA)

Ammonium persulfate (APS)

Ammonium sulfate $(\mathrm{NH} 4)_{2} \mathrm{SO} 4$

Aprotinin

Avidin-Peroxidase

Blasticidin

Bromophenol blue

Calcium Chloride $\left(\mathrm{CaCl}_{2}\right)$

Chelex (Chelating Ion Exchange Resin)

Chloroform

Cholera Toxin

Co-precipitant Pink

Colorless co-precipitant

Crystal violet

Cytric acid

dATP

DePeX mounting media

Diaminobenzidine substrate

Diethylpyrocarbonate (DEPC)

Dimethyl sulfoxide (DMSO)

Dithiothreitol (DTT)

DMEM

DMEM/F12

DMEM without Glucose

dNTPs

dNTPs
Carl Roth GmbH \& Co. KG, Karlsruhe

Carl Roth $\mathrm{GmbH} \&$ Co. KG, Karlsruhe

Carl Roth $\mathrm{GmbH}$ \& Co. KG, Karlsruhe

Carl Roth GmbH \& Co. KG, Karlsruhe

Carl Roth $\mathrm{GmbH} \&$ Co. KG, Karlsruhe

ExtrAvidin®-Peroxidase, Sigma-Aldrich, St. Louis, USA

Invitrogen $\mathrm{GmbH}$, Karlsruhe

Sigma-Aldrich Co., St. Louis, USA

Carl Roth $\mathrm{GmbH}$ \& Co. KG, Karlsruhe

Bio-Rad Laboratories GmbH, München

Carl Roth $\mathrm{GmbH}$ \& Co. KG, Karlsruhe

Sigma-Aldrich Co., St. Louis, USA

Bioline, Luckenwalde

Bioline, Luckenwalde

Sigma-Aldrich Co., St. Louis, USA

Carl Roth GmbH \& Co. KG, Karlsruhe

New England Biolabs, Frankfurt am Main

VWR International GmbH

ImmPACTTM DAB, SK-4105, Vector,

Burlingame, USA

Carl Roth GmbH \& Co. KG, Karlsruhe

AppliChem GmbH, Darmstadt

Carl Roth $\mathrm{GmbH} \&$ Co. KG, Karlsruhe

GIBCO®, Invitrogen $\mathrm{GmbH}$, Darmstadt

GIBCO®, Invitrogen $\mathrm{GmbH}$, Darmstadt

PAN Biotech GmbH, Aidenbach

Promega $\mathrm{GmbH}$, Mannheim

Carl Roth $\mathrm{GmbH} \&$ Co. KG, Karlsruhe 
Ethanol absolute

Ethidium bromide

Ethylenediaminetetraacetic acid (EDTA)

Epidermal Growth Factor (EGF)

Fetal Bovine Serum (FBS)

Formaldehyde

Glycerol

B-Glycerolphosphate (BGP)

Glycine

GlycoBlue

Guava Nexin® reagent

Hematoxyline (Mayer's hemalaun solution)

Horse Serum

Hydrochloric acid $(\mathrm{HCl})$

Hydrocortisone

Insulin

Isopropanol

Klenow fragment (3' to 5' exo-)

Leupeptin

Magnesium chloride ( $\mathrm{MgCl} 2)$

Methanol

N-ethylmaleimide (NEM)

NonidetTM P40 (NP-40)

Opti-MEM

PBS tablets

Pefabloc SC Protease Inhibitor

Penicillin-Streptomycin solution
Th. Geyer GmbH \& Co. KG, Renningen

Carl Roth $\mathrm{GmbH} \&$ Co. KG, Karlsruhe

Carl Roth $\mathrm{GmbH}$ \& Co. KG, Karlsruhe

Sigma-Aldrich Co., St. Louis, USA

Thermo Scientific HyClone, Logan, USA Material 28

Sigma-Aldrich Co., St. Louis, USA

Carl Roth $\mathrm{GmbH}$ \& Co. KG, Karlsruhe

Sigma-Aldrich Co., St. Louis, USA

Carl Roth $\mathrm{GmbH} \&$ Co. KG, Karlsruhe

Applied Biosystems/Ambion, Austin, USA

Millipore $\mathrm{GmbH}$, Schwalbach

Merck, Darmstadt

Sigma-Aldrich Co., St. Louis, USA

Carl Roth GmbH \& Co. KG, Karlsruhe

Sigma-Aldrich Co., St. Louis, USA

Sigma-Aldrich Co., St. Louis, USA

Carl Roth $\mathrm{GmbH}$ \& Co. KG, Karlsruhe

New England Biolabs, Frankfurt am Main

Carl Roth $\mathrm{GmbH}$ \& Co. KG, Karlsruhe

Carl Roth $\mathrm{GmbH}$ \& Co. KG, Karlsruhe

M. Baker B.V., Deventer, Netherlands

Sigma-Aldrich Co., St. Louis, USA

Sigma-Aldrich Co., St. Louis, USA

GIBCO®, Invitrogen $\mathrm{GmbH}$, Darmstadt

GIBCO®, Invitrogen $\mathrm{GmbH}$, Darmstadt

Carl Roth $\mathrm{GmbH} \&$ Co. KG, Karlsruhe

Sigma-Aldrich Co., St. Louis, USA 
Peptone

Potassium acetate (KOAc)

Potassium chloride $(\mathrm{KCl})$

Potassium dihydrogen phosphate $\left(\mathrm{KH}_{2} \mathrm{PO}_{4}\right)$

Propidium iodide solution

Protein A Sepharose CL-4B

RNase inhibitor

RNAiMAX

Roti@-Phenol

Rotiphorese® Gel 30

Rotipuran® Chloroform

Rotipuran® Isoamylalcohol

Salmon sperm DNA

SepharoseTM CL-4B

Skim milk powder

Sodium acetate

Sodium chloride $(\mathrm{NaCl})$

Sodium deoxycholate

Sodium dodecylsulfate (SDS)

di-Sodium hydrogen phosphate dihydrate

Sodium hydroxide $(\mathrm{NaOH})$

Sodium pyruvate (Na-Pyr)

SureClean

SYBR Green

TEMED

a, $\alpha$-Trehalose Dihydrate

Tris
Carl Roth $\mathrm{GmbH} \&$ Co. KG, Karlsruhe

Carl Roth $\mathrm{GmbH} \&$ Co. KG, Karlsruhe

AppliChem GmbH, Darmstadt

Carl Roth $\mathrm{GmbH}$ \& Co. KG, Karlsruhe

Sigma-Aldrich Co., St. Louis, USA

GE Healthcare, Uppsala, Sweden

New England Biolabs, Frankfurt am Main

Invitrogen $\mathrm{GmbH}$, Karlsruhe

Carl Roth $\mathrm{GmbH} \&$ Co. KG, Karlsruhe

Carl Roth $\mathrm{GmbH} \&$ Co. KG, Karlsruhe

Carl Roth $\mathrm{GmbH} \&$ Co. KG, Karlsruhe

Carl Roth $\mathrm{GmbH} \&$ Co. KG, Karlsruhe Material 29

Stratagene, La Jolla, USA

GE Healthcare, Uppsala, Sweden

Carl Roth $\mathrm{GmbH} \&$ Co. KG, Karlsruhe

Carl Roth $\mathrm{GmbH}$ \& Co. KG, Karlsruhe

Carl Roth $\mathrm{GmbH} \&$ Co. KG, Karlsruhe

AppliChem GmbH, Darmstadt

Carl Roth GmbH \& Co. KG, Karlsruhe

Carl Roth $\mathrm{GmbH}$ \& Co. KG, Karlsruhe

Carl Roth $\mathrm{GmbH} \&$ Co. KG, Karlsruhe

GIBCO®, Invitrogen $\mathrm{GmbH}$, Darmstadt

Bioline $\mathrm{GmbH}$, Luckenwalde

Roche Diagnostics $\mathrm{GmbH}$, Mannheim

Carl Roth $\mathrm{GmbH} \&$ Co. KG, Karlsruhe

USB Corporation, Cleveland, USA

Carl Roth GmbH \& Co. KG, Karlsruhe 
Triton X-100

TRIzol® Reagent

Trypsin-EDTA (0.05\%)

Tween-20

Xylene

Yeast Extract

Zeocin

\subsubsection{Inhibitors}

Acadesine (AICAR)

Actinomycin D

Cisplatin

CX-5461

Doxorubicin

lodacetamide

Metformin

$\mathrm{NiCl}_{2}$

Sorbitol

SP600125

\subsection{Kits and reagents}

ATP Determination Kit

Bioanalyzer DNA High Sensitivity Kit

Lipofectamine $^{\mathrm{TM}} 2000$

Lipofectamine $^{\text {TM }}$ RNAiMAX

PureYield $^{\mathrm{TM}}$

QIAprep® Spin Miniprep Kit
AppliChem GmbH, Darmstadt

Invitrogen $\mathrm{GmbH}$, Karlsruhe

GIBCO®, Invitrogen $\mathrm{GmbH}$, Darmstadt

AppliChem GmbH, Darmstadt

Carl Roth GmbH \& Co. KG, Karlsruhe

USB Corporation, Cleveland, USA

InvivoGen, San Diego, USA

Biaffin GmbH \& Co HG, Kassel

Sigma-Aldrich Co., St. Louis, USA

Teva GmbH, Ulm

Selleck, Houston, USA

Enzo Life Sciences GmbH, Lörrach

Sigma-Aldrich Co., St. Louis, USA

Sigma-Aldrich Co., St. Louis, USA

Sigma-Aldrich Co., St. Louis, USA

USB Corporation, Cleveland, USA

Sigma-Aldrich Co., St. Louis, USA

Invitrogen $\mathrm{GmbH}$, Karlsruhe

Agilent, Santa Clara, USA

Invitrogen $\mathrm{GmbH}$, Karlsruhe

Invitrogen $\mathrm{GmbH}$, Karlsruhe

Plasmid Midiprep Promega $\mathrm{GmbH}$, Mannheim

Qiagen $\mathrm{GmbH}$, Hilden 
Qubit dsDNA HS Assay

SuperSignal® West Dura

SuperSignal® West Femto Maximum
Invitrogen $\mathrm{GmbH}$, Karlsruhe

Thermo Fisher Scientific, Waltham, USA

Thermo Fisher Scientific, Waltham, USA

\subsection{Nucleic acids}

\subsubsection{Vectors and expression constructs}

\begin{tabular}{|l|l|}
\hline Name & Reference \\
\hline pcDNA4/TO & (Monroe et al., 2003) \\
\hline pcDNA4/TO-Flag-RNF40 & (Kari et al., 2012) \\
\hline
\end{tabular}

\subsubsection{Oligonucleotides}

\subsubsection{1 siRNA oligonucleotides}

Target Gene siRNA Target Sequence Source

\begin{tabular}{|l|l|l|l|}
\hline Target Gene & siRNA sequence 5‘-3‘ direction & Source & Cat.No. \\
\hline $\begin{array}{l}\text { siGENOME } \\
\text { Nontargeting } \\
\text { siRNA pool \# 1 }\end{array}$ & - & Dharmacon & D-001206-13 \\
\hline $\begin{array}{l}\text { Silencer® Select } \\
\text { Negative Control \#1 }\end{array}$ & - & Ambion & 4390844 \\
\hline $\begin{array}{l}\text { AMPK } \alpha 1 \\
\text { siGenome }(\# 1)\end{array}$ & CAAAGUCGACCAAAUGAUA & Dharmacon & D-005027-01 \\
\hline $\begin{array}{l}\text { AMPK } \alpha 1 \\
\text { siGenome (\#2) }\end{array}$ & GUAGAGCAAUCAAACAAUU & Dharmacon & D-005027-02 \\
\hline $\begin{array}{l}\text { AMPK } \alpha 1 \\
\text { siGenome (\#3) }\end{array}$ & GACAAGCACUUACUCCAAA & Dharmacon & D-005027-03 \\
\hline $\begin{array}{l}\text { AMPK } \alpha 1 \\
\text { siGenome (\#5) }\end{array}$ & ACAAUUGGAUUAUGAAUGG & Dharmacon & D-005027-05 \\
\hline $\begin{array}{l}\text { AMPK } \alpha 2 \\
\text { siGenome (\#2) }\end{array}$ & GUACCUACGUUAUUUAAGA & Dharmacon & D-005361-02 \\
\hline $\begin{array}{l}\text { AMPK } \alpha 2 \\
\text { siGenome (\#3) }\end{array}$ & GGAAGGUAGUGAAUGCAUA & Dharmacon & D-005361-03 \\
\hline $\begin{array}{l}\text { AMPK } \alpha 2 \\
\text { siGenome (\#4) }\end{array}$ & ACAGAAGAUUCGCAGUUUA & Dharmacon & D-005361-05 \\
\hline $\begin{array}{l}\text { AMPK } \alpha 2 \\
\text { siGenome (\#5) }\end{array}$ & AAAGAAACCUGAAGACAAU & Dharmacon & D-011106-02 \\
\hline $\begin{array}{l}\text { ATXN7 } \\
\text { siGenome (\#2) }\end{array}$ & CGAAGGCGAUGACAAAGAA & Dharmacon \\
\hline $\begin{array}{l}\text { ATXN7 } \\
\text { siGenome (\#3) }\end{array}$ & D-011106-03 \\
\hline
\end{tabular}




\begin{tabular}{|c|c|c|c|}
\hline $\begin{array}{l}\text { ATXN7 } \\
\text { siGenome (\#4) }\end{array}$ & CAGGAAACCUCAGAAAUUG & Dharmacon & D-011106-04 \\
\hline $\begin{array}{l}\text { ATXN7 } \\
\text { siGenome (\#17) }\end{array}$ & CCAAAUAGCAAAUCGGUAC & Dharmacon & D-011106-17 \\
\hline $\begin{array}{l}\text { ATXN7L3 } \\
\text { siGENOME (\# 1) }\end{array}$ & GUCGAGAGCUCCCUGGAUA & Dharmacon & D-023237-01 \\
\hline $\begin{array}{l}\text { ATXN7L3 } \\
\text { siGENOME (\# 2) }\end{array}$ & GGUCGAGGAUUCUUGUUUG & Dharmacon & D-023237-02 \\
\hline $\begin{array}{l}\text { ATXN7L3 } \\
\text { siGENOME (\# 3) }\end{array}$ & UGAAUAAGUCUGAGAGUGA & Dharmacon & D-023237-03 \\
\hline $\begin{array}{l}\text { ATXN7L3 } \\
\text { siGENOME (\# 4) }\end{array}$ & GAGGCGAACCGUACGGAUU & Dharmacon & D-023237-04 \\
\hline $\begin{array}{l}\text { ENY2 } \\
\text { siGENOME (\# 1) }\end{array}$ & GAGAACGCCUCAAAGAGUU & Dharmacon & D-018808-01 \\
\hline $\begin{array}{l}\text { ENY2 } \\
\text { siGENOME (\# 2) }\end{array}$ & GAGCAGCGAUUAACCAAAA & Dharmacon & D-018808-02 \\
\hline $\begin{array}{l}\text { ENY2 } \\
\text { siGENOME (\# 3) }\end{array}$ & CCUGGUACCUGACAGUGUA & Dharmacon & D-018808-03 \\
\hline $\begin{array}{l}\text { ENY2 } \\
\text { siGENOME (\# 4) }\end{array}$ & GGCACACUGUAAAGAGGUA & Dharmacon & D-018808-04 \\
\hline $\begin{array}{l}\text { LKB1 } \\
\text { siGenome (\#1) }\end{array}$ & UGAAAGGGAUGCUUGAGUA & Dharmacon & D-005035-01 \\
\hline $\begin{array}{l}\text { LKB1 } \\
\text { siGenome (\#3) }\end{array}$ & GAGAAGCGUUUCCCAGUGU & Dharmacon & D-005035-03 \\
\hline $\begin{array}{l}\text { LKB1 } \\
\text { siGenome (\#5) }\end{array}$ & GCUCUUACGGCAAGGUGAA & Dharmacon & D-005035-05 \\
\hline $\begin{array}{l}\text { LKB1 } \\
\text { siGenome (\#6) }\end{array}$ & GCAUGACUGUGGUGCCGUA & Dharmacon & D-005035-06 \\
\hline RNF20 & AGCUCUUAUCCCGGAAGCUAA & Ambion & S32087 \\
\hline RNF20 & CAGGUCCGCAAGGAGUAUGAA & Ambion & S32088 \\
\hline RNF40 & AGAGAUGGAUGUGACAGGUCA & Ambion & S18960 \\
\hline RNF40 & AGGUACUACAGUUCAAGAACA & Ambion & S18962 \\
\hline $\begin{array}{l}\text { RNF40 } \\
\text { siGENOME (\# 1) }\end{array}$ & GAGAUGCGCCACCUGAUUAUU & Dharmacon & D-006913-01 \\
\hline $\begin{array}{l}\text { RNF40 } \\
\text { SiGENOME (\# 2) }\end{array}$ & GAUGCCAACUUUAAGCUAAUU & Dharmacon & D-006913-02 \\
\hline $\begin{array}{l}\text { RNF40 } \\
\text { siGENOME (\# 3) }\end{array}$ & GAUCAAGGCCAACCAGAUUUU & Dharmacon & D-006913-03 \\
\hline $\begin{array}{l}\text { RNF40 } \\
\text { siGENOME (\# 4) }\end{array}$ & CAACGAGUCUCUGCAAGUGUU & Dharmacon & D-006913-04 \\
\hline USP22 & GGAGAGAAGUUUUCAACUU & Ambion & S23566 \\
\hline $\begin{array}{l}\text { USP22 } \\
\text { siGENOME (\# 1) }\end{array}$ & CCUUUAGUCUCAAGAGCGA & Dharmacon & D-006072-01 \\
\hline $\begin{array}{l}\text { USP22 } \\
\text { siGENOME (\# 2) }\end{array}$ & GGAAGAUCACCACGUAUGU & Dharmacon & D-006072-02 \\
\hline $\begin{array}{l}\text { USP22 } \\
\text { siGENOME (\# 3) }\end{array}$ & CAAAGCAGCUCACUAUGAA & Dharmacon & D-006072-03 \\
\hline
\end{tabular}




\begin{tabular}{|l|l|l|l|}
\hline $\begin{array}{l}\text { USP22 } \\
\text { siGENOME (\# 4) }\end{array}$ & GGAGAAAGAUCACCUCGAA & Dharmacon & D-006072-04 \\
\hline
\end{tabular}

For transfections the Dharmacon siRNAs (\#1 - \#4) were pooled in a 1:1:1:1 ratio.

\subsubsection{RT-PCR primers}

Reverse transcription primers from Metabion AG, Martinsried.

\subsubsection{3 qPCR primers}

Primers utilized in qPCR in 5' to 3' orientation. Primers were obtained from previous studies or designed using a primer designing tool program (www.ncbi.nlm.nih.gov/tools/primerblast/).

\begin{tabular}{|l|l|l|}
\hline Name & 5'-3' sequence $^{\prime}$ & Reference \\
\hline 28S rRNA & GGGTAAGAAGCCATCAACCAAC & (Prenzel et al., 2011) \\
\hline 28S rRNA & ATCAACCAACACCTTTTCTG & (Prenzel et al., 2011) \\
\hline 5.8S-ITS2 F & CCGGGGCTACGCCTGTCTGA & this study \\
\hline 5.8S-ITS2 R & TGCGCTTAGGGGGACGGAGG & this study \\
\hline 5'ETS-18S F & GCTCGCCGCGCTCTACCTTAC & this study \\
\hline 5'ETS-18S R & TTTCACTGTACCGGCCGTGCG & this study \\
\hline ABCC2 F & CACATGCTCCAGGCCAGTGTTTC & this study \\
\hline ABCC2 F (hnRNA) & CAGGGATTCCTGTGCATGGTGCTG & this study \\
\hline ABCC2 F $($ hnRNA) & CCGGCCTGTGGGTGTTGTGTC & this study \\
\hline ABCC2 R & TCCAGGTTCACATCTCGGACTGTGG & this study \\
\hline ATXN7L3 F & GTTGCCGCCCGCTGTGCTAT & this study \\
\hline ATXN7L3 R & AAGCCCGGCTGGTCCACGAT & this study \\
\hline ENY2 F 1605 & CCAACTGGTGCTCATGCTGCTGATT & this study \\
\hline ENY2 R 1791 & AGGCCACTTAGGCAACAGTGTGGA & this study \\
\hline FST F & ACCTACTCCAGTGCCTGCCACC & this study \\
\hline FST F $($ hnRNA) & CCCGAAGCCCTCCTGGCTGA & this study \\
\hline FST R & CACAGAGGGAACACCGGCCTCT & this study \\
\hline FST R (hnRNA) & AACAATCCGGGGCGCAGACG & this study \\
\hline IL1B F & CTGAACTGCACGCTCCGGGAC & this study \\
\hline IL1B F (hnRNA) & TGGGGTGACCCTGCACTGCT & this study \\
\hline IL1B R & CTTGAGGCCCAAGGCCACAGGT & this study \\
\hline IL1B R (hnRNA) & TGAGGCCCAAGGCCACAGGT & this study \\
\hline ITS2-28S F & GCGATTCCGTCCGTCCGTCC & this study \\
\hline ITS2-28S R & AGCGGGTCGCCACGTCTGAT & this study \\
\hline RNF20 F & TGGCCAAGCAGGAAGAAG & (Karpiuk et al., 2012) \\
\hline RNF20 R & ACGCTCTGACATGAGCTTGA & (Karpiuk et al., 2012) \\
\hline RNF40 F & AGTACAAGGCGCGGTTGA & (Prenzel et al., 2011) \\
\hline RNF40 R & GAAGCAGAAAACGTGGAAGC & (Prenzel et al., 2011) \\
\hline RPLP0 F & GATTGGCTACCCAACTGTTG & (Fritah et al., 2005) \\
\hline
\end{tabular}




\begin{tabular}{|l|l|l|}
\hline RPLP0 F (hnRNA) & AGATCAGGGACATGTTGCTGGCCA & this study \\
\hline RPLP0 R & CAGGGGCAGCAGCCACAAA & (Fritah et al., 2005) \\
\hline RPLP0 R (hnRNA) & AATGGCACCAGCACGGGCAG & this study \\
\hline USP22 F & AGCCAAGGGTGTTGGTCGCG & this study \\
\hline USP22 R & ACTGCCACCACGCCCGAAAG & this study \\
\hline
\end{tabular}

hnRNA = heterogeneous nuclear RNA

\subsubsection{ChIP primers}

Primers utilized in ChIP in $5^{\prime \prime}$ to $3^{\prime \prime}$ orientation.

\begin{tabular}{|l|l|l|}
\hline Name & 5'-3‘ sequence & Reference \\
\hline ABCC $+1923 \mathrm{~F}$ & TCCGGAGGCAGACCTGCCACT & this study \\
\hline ABCC $+2029 \mathrm{R}$ & ATTTATACACGTGGAGAAGCTGCCAGGG & this study \\
\hline ABCC2 $+138 \mathrm{R}$ & ATTCCTGGACTGCGTCTGGAACGA & this study \\
\hline ABCC2 $-5 \mathrm{~F}$ & GGTTGGGATGAAAGGTCATCCTTTACGGA & this study \\
\hline FST +116 R & TTACCCTGGGCACTGCGGTCC & this study \\
\hline FST +2018F & CATCCCCGCCGGGTCTCCTT & this study \\
\hline FST +2137R & TGACGGAGCCAGCAGTTCCCA & this study \\
\hline FST +67 F & CTGCTGCTGCTGCTCTGCCAG & this study \\
\hline GAPDH +1061F & CCGGGAGAAGCTGAGTCATG & (Shema et al., 2008) \\
\hline GAPDH +1111R & TTTGCGGTGGAATGTCCTT & (Shema et al., 2008) \\
\hline GAPDH +67R & AAGAAGATGCGGCTGACTGT & this study \\
\hline GAPDH -122F & CGGCTACTAGCGGTTTTACG & this study \\
\hline IL1B +1137F & TGGCAGTTCCTACAACGGGAGAACC & this study \\
\hline IL1B +1234R & AAAGCCCTGTCTGGGTGGCCTAG & this study \\
\hline IL1B +3329F & CTCCAGCTCTCCTAGCCAATACATTGCT & this study \\
\hline IL1B +3473R & TCAGCATCCAGTGCATGGCAGTCA & this study \\
\hline IL1B +70R & CGCTCAGGCCACAGCACTTCC & this study \\
\hline IL1B -81F & GCTCCCCATCTCTGCTTGTTCCACT & this study \\
\hline RPLP0 +2439F & CAGGTGTTACTGCCCCACT & this study \\
\hline RPLP0 +2609R & AGCACAGCCTGGATAGCAAC & this study \\
\hline RPLP0 +57 R & CAATCAGAAACCGCGGATAG & this study \\
\hline RPLP0 -85 F & CTTCGCGACCCTACTTAAAGG & this study \\
\hline
\end{tabular}

\subsubsection{Primers for ChIP-seq library preparation}

\begin{tabular}{|l|l|l|}
\hline Name & $\mathbf{5}^{\star}-3^{\star}$ sequence & Reference \\
\hline Multi-Adap1 F & Phosphate-GATCGGAAGAGCACACGTCT & Illumina \\
\hline Multi-Adapt1 R & $\begin{array}{l}\text { ACACTCTTTCCCTACACGACGCTCTTCCGAT } \\
C^{*} T\end{array}$ & Illumina \\
\hline Multi-PCR-1.0 & $\begin{array}{l}\text { AATGATACGGCGACCACCGAGATCTACACT } \\
\text { CTTTCCCTACACGACGCTCTTCCGATC*T }\end{array}$ & modified from Illumina \\
\hline
\end{tabular}




\begin{tabular}{|c|c|c|}
\hline Multi-PCR-Ind1 & $\begin{array}{l}\text { CAAGCAGAAGACGGCATACGAGATCGTGAT } \\
\text { GTGACTGGAGTTCAGACGTGTGCTCTTCCG } \\
\text { ATC*T }\end{array}$ & modified from Illumina \\
\hline Multi-PCR-Ind2 & 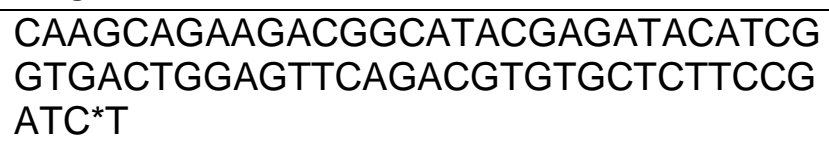 & modified from Illumina \\
\hline Multi-PCR-Ind3 & $\begin{array}{l}\text { CAAGCAGAAGACGGCATACGAGATGCCTAA } \\
\text { GTGACTGGAGTTCAGACGTGTGCTCTTCCG } \\
\text { ATC*T }\end{array}$ & modified from Illumina \\
\hline Multi-PCR-Ind4 & $\begin{array}{l}\text { CAAGCAGAAGACGGCATACGAGATTGGTCA } \\
\text { GTGACTGGAGTTCAGACGTGTGCTCTTCCG } \\
\text { ATC }^{*} T\end{array}$ & modified from Illumina \\
\hline Multi-PCR-Ind6 & $\begin{array}{l}\text { CAAGCAGAAGACGGCATACGAGATATTGGC } \\
\text { GTGACTGGAGTTCAGACGTGTGCTCTTCCG } \\
\text { ATC*T }\end{array}$ & modified from Illumina \\
\hline Multi-PCR-Ind7 & $\begin{array}{l}\text { CAAGCAGAAGACGGCATACGAGATGATCTG } \\
\text { GTGACTGGAGTTCAGACGTGTGCTCTTCCG } \\
\text { ATC*T }\end{array}$ & modified from Illumina \\
\hline Multi-PCR-Ind8 & $\begin{array}{l}\text { CAAGCAGAAGACGGCATACGAGATTCAAGT } \\
\text { GTGACTGGAGTTCAGACGTGTGCTCTTCCG } \\
\text { ATC*T }\end{array}$ & modified from Illumina \\
\hline Multi-PCR-Ind9 & $\begin{array}{l}\text { CAAGCAGAAGACGGCATACGAGATCTGATC } \\
\text { GTGACTGGAGTTCAGACGTGTGCTCTTCCG } \\
{\text { ATC }{ }^{*} T}\end{array}$ & modified from Illumina \\
\hline Multi-PCR-Ind10 & $\begin{array}{l}\text { CAAGCAGAAGACGGCATACGAGATAAGCTA } \\
\text { GTGACTGGAGTTCAGACGTGTGCTCTTCCG } \\
\text { ATC }^{*} T\end{array}$ & modified from Illumina \\
\hline Multi-PCR-Ind12 & $\begin{array}{l}\text { CAAGCAGAAGACGGCATACGAGATTACAAG } \\
\text { GTGACTGGAGTTCAGACGTGTGCTCTTCCG } \\
\text { ATC*T }\end{array}$ & modified from Illumina \\
\hline
\end{tabular}

\subsection{Proteins}

\subsubsection{Molecular weight standards}

Gene Ruler ${ }^{\mathrm{TM}}$ DNA-Ladder

PageRuler ${ }^{\mathrm{TM}}$ Prestained Protein Ladder
Fermentas $\mathrm{GmbH}$, St. Leon-Rot

Fermentas $\mathrm{GmbH}$, St. Leon-Rot

\subsubsection{Enzymes}

Klenow DNA Polymerase

Phusion Polymerase

Proteinase $\mathrm{K}$

Quick Ligase
New England Biolabs, Frankfurt am Main New England Biolabs, Frankfurt am Main Invitrogen $\mathrm{GmbH}$, Karlsruhe New England Biolabs, Frankfurt am Main 
Restriction enzymes

Reverse Transcriptase (M-MuLV)

RNase A

Streptavidin Peroxidase

Taq DNA Polymerase

T4 Polynucleotide Kinase

T4 DNA Ligase
New England Biolabs, Frankfurt am Main New England Biolabs, Frankfurt am Main Qiagen $\mathrm{GmbH}$, Hilden

Sigma-Aldrich Co., St. Louis, USA

Prime Tech, Minsk, Belarus

New England Biolabs, Frankfurt am Main

New England Biolabs, Frankfurt am Main

\subsubsection{Antibodies}

\subsubsection{Primary antibodies}

Antibodies used for ChIP and Western blot analyses and the respective dilutions.

\begin{tabular}{|c|c|c|c|c|c|}
\hline Name & Clone & Cat. Number & WB & ChIP & Source \\
\hline ATXN7L3 & - & PA5-32017 & $1: 1000$ & - & Thermo Scientific \\
\hline H2Aub1 & D27C4 & 8240 & $1: 1000$ & - & Cell Signaling \\
\hline $\mathrm{H} 2 \mathrm{~B}$ & - & $07-371$ & $1: 3000$ & - & Millipore \\
\hline H2Bub1 & 56 & $05-1312$ & $1: 5000$ & $2 \mu g$ & $\begin{array}{l}\text { Millipore (for IHC } \\
\text { and WB) }\end{array}$ \\
\hline H2Bub1 & 7B4 & - & $1: 100$ & - & $\begin{array}{l}\text { (Prenzel et al., } \\
2011 \text { ) }\end{array}$ \\
\hline H2Bub1 & $\mathrm{D} 11$ & 5546 & - & $2 \mu \mathrm{l}$ & $\begin{array}{l}\text { Cell Signaling (for } \\
\text { ChIP) }\end{array}$ \\
\hline H3K36me3 & - & $a b 9050-100$ & - & $1 \mu g$ & Abcam \\
\hline HSC70 & B-6 & sc-7298 & $1: 25000$ & - & Santa Cruz \\
\hline $\begin{array}{l}\text { IgG (non- } \\
\text { specific) }\end{array}$ & - & $a b 46540$ & - & $1 \mu \mathrm{g}$ & Abcam \\
\hline$p-J N K$ & - & 46685 & $1: 1000$ & - & Cell Signaling \\
\hline RNAPII & CTD4H8 & MMS-128P & - & $1 \mu \mathrm{g}$ & Covance \\
\hline RNF40 & KA7-27 & R9029 & $1: 1000$ & - & Sigma \\
\hline USP22 & - & NBP1-49549 & $1: 1000$ & - & Novus Biologicals \\
\hline
\end{tabular}




\begin{tabular}{|l|l|l|l|l|l|}
\hline USP22 & - & $\begin{array}{l}\text { HPA044980- } \\
100 \mathrm{UL}\end{array}$ & $1: 1000$ & $1 \mu \mathrm{g}$ & $\begin{array}{l}\text { Sigma-Aldrich } \\
\text { Prestige }\end{array}$ \\
\hline$\beta$-Actin & - & $\mathrm{ab6276-100}$ & $1: 10000$ & - & Abcam \\
\hline
\end{tabular}

\subsubsection{Secondary antibodies}

\begin{tabular}{|l|l|l|l|}
\hline Name & $\begin{array}{l}\text { Cat. } \\
\text { Number }\end{array}$ & WB & Source \\
\hline Donkey Anti-Mouse IgG-HRP & $715-036-150$ & $1: 10000$ & $\begin{array}{l}\text { Jackson } \\
\text { ImmunoResearch }\end{array}$ \\
\hline Donkey Anti-Rabbit IgG-HRP & $711-036-152$ & $1: 10000$ & $\begin{array}{l}\text { Jackson } \\
\text { ImmunoResearch }\end{array}$ \\
\hline
\end{tabular}

\subsection{Cells}

\subsubsection{Bacteria cells}

Escherichia coli DH10BTM from Invitrogen $\mathrm{GmbH}$, Karlsruhe.

\subsubsection{Eukaryotic cell lines}

\begin{tabular}{|l|l|l|l|l|}
\hline Species & Organ & $\begin{array}{l}\text { Tissue } \\
\text { origin }\end{array}$ & Disease & Source \\
\hline H1299 & human & lung & carcinoma & $\begin{array}{l}\text { Prof. M. Dobbelstein, } \\
\text { GZMB, Göttingen }\end{array}$ \\
\hline HCT116 wildtype & human & colon & carcinoma & $\begin{array}{l}\text { Prof. M. Dobbelstein, } \\
\text { GZMB, Göttingen }\end{array}$ \\
\hline MCF10A & human & breast & $\begin{array}{l}\text { nontumorigenic } \\
\text { epithelial cell line }\end{array}$ & $\begin{array}{l}\text { Prof. M. Oren } \\
\text { (Weizmann Institute of } \\
\text { Science, Rehovot, Israel) }\end{array}$ \\
\hline U2OS & human & bone & osteosaroma & $\begin{array}{l}\text { Prof. M. Dobbelstein, } \\
\text { GZMB, Göttingen }\end{array}$ \\
\hline $\begin{array}{l}\text { U2OS with stable Tet-R } \\
\text { overexpression }\end{array}$ & human & bone & osteosaroma & $\begin{array}{l}\text { Prof. T. Spelsberg, Mayo } \\
\text { Clinic, USA }\end{array}$ \\
\hline $\begin{array}{l}\text { U2OS with stable Tet-R- } \\
\text { RNF40 overexpression }\end{array}$ & human & bone & osteosaroma & $\begin{array}{l}\text { Prof. T. Spelsberg, Mayo } \\
\text { Clinic, USA }\end{array}$ \\
\hline
\end{tabular}




\subsection{Buffers and solutions}

\section{RIPA buffer}

PBS $1 X$

NP40 1\%

Sodiumdeoxycholate $0.5 \%$

SDS $0.1 \%$

\section{X TBS-T}

Tris $0.1 \mathrm{M}$

$\mathrm{NaCl} 1.5 \mathrm{M}$

Tween-20 0.5\%

\section{Western salts 10X}

Tris $0.25 \mathrm{M}$

Glycin $0.86 \mathrm{M}$

SDS $0.7 \mathrm{mM}$

\section{PBS-T 10X (pH 7,4)}

$\mathrm{NaCl} 0.73 \mathrm{M}$

KCl $0.027 \mathrm{M}$

$\mathrm{NaH}_{2} \mathrm{PO}_{4}{ }^{\star} 7 \mathrm{H}_{2} \mathrm{O} 14.3 \mathrm{mM}$

$\mathrm{KH}_{2} \mathrm{PO}_{4} 14.7 \mathrm{mM}$

Tween 20 1\%

\section{PBS++}

$1 \times$ PBS

$\mathrm{CaCl}_{2} 0.9 \mathrm{mM}$

$\mathrm{MgCl}_{2} 0.5 \mathrm{mM}$ 


\section{PBS for cell culture}

1 PBS tablet per $500 \mathrm{ml} \mathrm{ddH} 2 \mathrm{O}$

\section{PBS-T}

PBS including $0.1 \%(w / v)$ Tween-20

\section{PCR-Mix 10X}

Tris-HCl (pH 8.8) 750 mM

$\left(\mathrm{NH}_{4}\right)_{2} \mathbf{S O}_{4} 200 \mathrm{mM}$

Tween-20 0.1\%

\section{q-RT-PCR master mix}

PCR-Mix $1 X$

$\mathrm{MgCl}_{2} 3 \mathrm{mM}$

SYBR Green 1:80000

dNTPs $0.2 \mathrm{mM}$

Taq-polymerase $20 \mathrm{U} / \mathrm{ml}$

Triton X-100 0.25\%

Trehalose $300 \mathrm{mM}$

\section{Pagano buffer}

Tris-HCl (pH 7.4) 20 mM

DTT 2 mM

EDTA $0.25 \mathrm{mM}$

\section{Deubiquitination buffer}

Tris-HCl (pH 7.4) 60 mM

Glycerol 20\%

$\mathrm{MgCl}_{2} 10 \mathrm{mM}$ 


\section{Blocking solution}

PBS-T 1X

Milk 5\%

\section{Cell culture freezing medium}

\section{DMEM}

FBS $50 \%$

DMSO 8\%

Chelex (10\%)

Chelex $10 \%$ (w/v) in $\mathrm{H} 2 \mathrm{O}$

Nelson buffer (modified)

$\mathrm{NaCl} 150 \mathrm{mM}$

EDTA pH 8.020 mM

Tris-HCl pH 7.550 mM

NP-40 0.5\%

Triton X-100 1\%

NaF $20 \mathrm{mM}$

Wienmann lysis buffer

Tris- $\mathrm{HCl}$ pH 8.050 mM

EDTA $10 \mathrm{mM}$

SDS $1 \%(w t / v o l)$ 


\section{ChIP dilution buffer (CDB)}

SDS $0.01 \%(\mathrm{wt} / \mathrm{vol})$

Triton X-100 1.1\% (vol/vol)

EDTA $1.2 \mathrm{mM}$

Tris-HCl pH 8.116 .7 mM

$\mathrm{NaCl} 167 \mathrm{mM}$

\section{Low-salt wash buffer}

SDS $0.1 \%(\mathrm{wt} / \mathrm{vol})$

Triton X-100 1\% (vol/vol)

EDTA 2 mM

Tris-HCl, pH 8.120 mM

$\mathrm{NaCl} 150 \mathrm{mM}$

\section{Lithium chloride wash buffer}

LiCl $0.25 \mathrm{M}$

NP40 1\% (vol/vol)

Sodium deoxycholate $1 \%(\mathrm{wt} / \mathrm{vol})$

EDTA $1 \mathrm{mM}$

Tris-HCl, pH 8.110 mM

\section{TE buffer}

Tris- $\mathrm{HCl}$ pH $8.010 \mathrm{mM}$

EDTA $1 \mathrm{mM}$

2X Hybridization buffer

Tris-HCl pH $7.520 \mathrm{mM}$

$\mathrm{NaCl} 20 \mathrm{mM}$ 
Protease, phosphatase and deubiquitinase inhibitors

Pefabloc $1 \mathrm{mM}$

Aprotinin/Leupeptin $1 \mathrm{ng} / \mu \mathrm{l}$

BGP $10 \mathrm{mM}$

NEM $1 \mathrm{mM}$

IAA $10 \mu \mathrm{M}$

$\mathrm{NiCl}_{2} 1 \mathrm{mM}$

DMEM cell culture "normal" medium

Phenol red-free, high-glucose DMEM

FBS $10 \%$

Penicillin $100 \mathrm{U} / \mathrm{ml}$

Streptomycin $100 \mu \mathrm{g} / \mathrm{ml}$

Sodium pyruvate $1 \mathrm{mM}$

DMEM/F12 cell culture medium

Phenol red-free, high-glucose DMEM/F12

Horse serum $5 \%$

EGF $100 \mu \mathrm{g} / \mathrm{ml}$

Hydrocortisone $1 \mathrm{mg} / \mathrm{ml}$

Cholera toxin $1 \mathrm{mg} / \mathrm{ml}$

Insulin $10 \mathrm{mg} / \mathrm{ml}$

Penicillin $100 \mathrm{U} / \mathrm{ml}$

Streptomycin $100 \mu \mathrm{g} / \mathrm{ml}$ 
6X Laemmli buffer

Tris (pH 6.8) $0.35 \mathrm{M}$

Glycerol 30\%

SDS $10 \%$

DTT $9.3 \%$

Bromphenol blue 0.02\%

\section{LB Agar}

LB medium 1X

Agar 1.5\%

\section{$\underline{\text { LB medium }}$}

Peptone 1\%

Yeast extract $0.5 \%$

$\mathrm{NaCl} 86 \mathrm{mM}$

SDS separating gel ( $X \%)$

Acrylamide $\mathrm{X} \%$

Tris-HCl (pH 8.8) 375 mM

SDS $0.1 \%$

APS $0.1 \%$

TEMED $0.04 \%$

SDS stacking gel (5\%)

Acrylamide 5\%

Tris-HCl (pH 6.8) 125.5 mM

SDS $0.1 \%$

APS $0.1 \%$

TEMED $0.1 \%$ 


\section{TAE buffer (50X)}

Tris $2 \mathrm{M}$

Acetic acid $1 \mathrm{M}$

EDTA $0.1 \mathrm{M}$

\section{$\underline{\text { Transfer buffer }}$}

10x Western salts $10 \%$

Methanol 15\%

\section{Citric acid buffer}

Citric acid (pH 6.0) 10 mM

\subsection{Software}

ModFit

Primer designing tool

ImageJ

ChIP-seq analysis
Verity Software House, Topsham, USA

NCBI/Primer-BLAST

(www.ncbi.nlm.nih.gov/tools/primer-blast/)

http://rsbweb.nih.gov/ij/

European Nucleotide Archive (http://www.ebi.ac.uk/ena/; study accession number SRP007976)

FastQC

(www.bioinformatics.babraham.ac.uk/projects/fas tqc/).

Bowtie (http://bowtie-bio.sourceforge.net) 


\section{Methods}

\subsection{Cell culture}

\subsubsection{Culturing cells}

H1299 (human non-small cell lung carcinoma), HCT116 (human colon carcinoma) and U2OS (human osteosarcoma) cells were cultured in phenol red-free highglucose Dulbecco's modified Eagles medium (DMEM) supplemented with 10\% fetal bovine serum (FBS), 100 units $/ \mathrm{ml}$ penicillin, $100 \mu \mathrm{g} / \mathrm{ml}$ streptomycin and $1 \mathrm{mM}$ sodium pyruvate at $37^{\circ} \mathrm{C}$ under $5 \% \mathrm{CO} 2$ atmosphere. MCF10A (non-transformed epithelial cell line derived from human fibrocystic mammary tissue) cells were

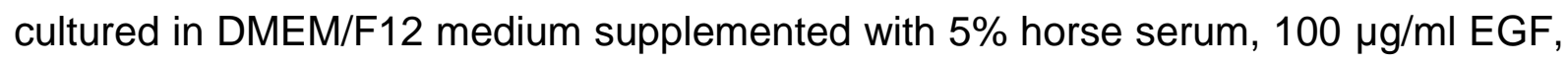
$1 \mathrm{mg} / \mathrm{ml}$ hydrocortisone, $1 \mathrm{mg} / \mathrm{ml}$ Cholera toxin, $10 \mathrm{mg} / \mathrm{ml}$ Insulin, 100 units $/ \mathrm{ml}$ penicillin and $100 \mu \mathrm{g} / \mathrm{ml}$ streptomycin under the same conditions. To culture U2OS TetR-RNF40 cells selective media containing Blasticidin $(5 \mathrm{mg} / \mathrm{ml})$ and Zeocin $(500$ $\mathrm{mg} / \mathrm{l})$ was used. For the induction of the transgene cells were grown in the presence of $1 \mu \mathrm{g} / \mathrm{ml}$ doxycycline.

\subsubsection{Reverse-transfection with siRNA}

siRNA transfections were performed using Lipofectamine RNAiMAX according to the manufacturer's instructions. For transfections in 6-well plate format for each well 30 pmol of respective siRNAs were mixed with $500 \mu \mathrm{l}$ of OptiMEM media. Then $5 \mu \mathrm{l}$ Lipofectamine RNAiMAX were added and incubated for 10-20 min at RT. In the meantime H1299 or MCF10A cells were trypsinized and diluted in medium without antibiotics so that $2.5 \mathrm{ml}$ contain 250,000 cells (MCF10A) or 150,000 cells (H1299). $2.5 \mathrm{ml}$ of the diluted cells were added to each well already containing the siRNALipofectamine RNAiMAX complexes. The medium was replaced with a fresh one after $24 \mathrm{~h}$. Cells were harvested $48 \mathrm{~h}$ after transfection. For transfections in $10 \mathrm{~cm}$ format reagent amounts and cell number were scaled up four times.

\subsubsection{Colony formation assay}

Different number of cells depending on cell line and experiment were seeded in a 6well plate. In order to analyze the effects of inducible RNF40 overexpression on the 
proliferation of U2OS-Tet-R cells, cells were treated with doxycycline the following day. The medium was changed and doxycycline was added every second day. To analyze the proliferation of breast cancer and osteosarcoma cells upon siRNAmediated knockdown cells were transfected and after $24 \mathrm{~h}$ the respective number of cells were re-plated into a 6 -well plate. After 8 days colonies were fixed with $70 \%$ methanol for $30 \mathrm{~min}$ on ice and then stained with $0.1 \%$ crystal violet solution $(0.1 \%$ $(\mathrm{w} / \mathrm{v})$ crystal violet, $10 \%(\mathrm{v} / \mathrm{v})$ formaldehyde) overnight. Stained colonies were rinsed thoroughly with water and then scanned with CanoScan 8600F.

\subsubsection{Migration assay}

Cell migration was assayed by seeding 100,000 MCF10A cells 48 hours after transfection with the respective siRNAs into $8.0 \mu \mathrm{m}$ PET track-etched membrane cell culture inserts. The inserts were pre-equilibrate for at least $30 \mathrm{~min}$ with serum free medium. After pre-equilibration the medium was substituted by normal MCF10A cell culture medium either with or without $20 \mathrm{ng} / \mathrm{ml}$ EGF to induce cell migration. Cells were grown for another $48 \mathrm{~h}$ and were scraped gently from the upper layer of the inserts using a Q-Tip before fixation with $100 \%$ methanol for $10 \mathrm{~min}$. Migrated cells were visualized by crystal violet staining for $10 \mathrm{~min}$. Then, the inserts were rinsed twice in distilled water to remove excess staining.

\subsubsection{Measurement of DNA of single cells by flow cytometry}

In order to analyze the effects of RNF40 overexpression and siRNA-mediated knockdown of RNF20 and RNF40 on cell cycle progression, DNA content was measured via flow cytometry. MCF10A and U2OS cells were washed twice with PBS, harvested by trypsinization and pelleted by centrifugation. After resuspending the cells in $0.5 \mathrm{ml}$ of PBS++, ice-cold ethanol was added dropwise to a final concentration of $75 \%$ ethanol. Cells were fixed overnight at $4^{\circ} \mathrm{C}$. Before staining, the cells were centrifuged, rehydrated in PBS++ for $10 \mathrm{~min}$, resuspended in PBS++ containing $0.5 \mathrm{mg} / \mathrm{ml}$ RNAse $A$ and incubated at $37^{\circ} \mathrm{C}$ for $30 \mathrm{~min}$. Cells were stained with $15 \mu \mathrm{l}$ propidium iodide $(1 \mathrm{mg} / \mathrm{ml})$ and flow cytometry analysis was performed using the Guava EasyCyte plus FACScan. About 10,000 cells were analyzed for each sample. Distribution of cells in distinct cell cycle phases was determined and graphically displayed using ModFIT cell cycle analysis software. 


\subsubsection{ATP determination assay}

MCF10A cells were cultured in a 6-well plate in normal or glucose free medium with and without 2-Deoxy-glucose. The medium was aspirated and the cells were washed twice with PBS with subsequent suspension in boiling water $(1.0 \mathrm{ml} / \mathrm{well})$ by repeated pipetting. The cell suspension was then transferred into a microcentrifuge tube for centrifugation $\left(12,000 \mathrm{~g}\right.$ for $5 \mathrm{~min}$ at $\left.4^{\circ} \mathrm{C}\right)$ and $20 \mu \mathrm{l}$ of the supernatant was used for bioluminescence measurement. The ATP assay was performed using an ATP determination kit according to the manufacturer's instructions. The standard curve of ATP was obtained by serial dilutions of $10 \mu \mathrm{M}$ ATP solution.

\subsection{Molecular biology}

\subsubsection{RNA isolation}

RNA isolation was performed with QIAzoI ${ }^{\circledR}$ reagent according to manufacturer's instructions. Cells were washed with PBS, lysed by addition of $1 \mathrm{ml}$ of QIAzol $\circledast$ reagent to each well (6-well format) and collected into $1.5 \mathrm{ml}$ tubes. After addition of $200 \mu \mathrm{l}$ of chloroform samples were vortexed and centrifuged at $10,000 \mathrm{~g}$ for $20 \mathrm{~min}$ $\left(4^{\circ} \mathrm{C}\right)$. After that the aqueous phase was collected and chloroform extraction was performed a second time followed by overnight isopropanol precipitation at $-20^{\circ} \mathrm{C}$. Then, samples were centrifuged at maximal speed of $12,000 \mathrm{~g}$ for $20 \min \left(4^{\circ} \mathrm{C}\right)$, pellets were washed twice with $70 \%$ ethanol, dried on vacuum concentrator and redissolved in $50 \mu \mathrm{l}$ of DEPC water. RNA concentration was measured using a NanoDrop.

\subsection{2 cDNA synthesis}

For DNA synthesis $1 \mu \mathrm{g}$ of total RNA was mixed with $2 \mu \mathrm{l}$ of $15 \mu \mathrm{M}$ random primers and $4 \mu \mathrm{l}$ of $2.5 \mathrm{mM}$ dNTP mix and incubated $5 \mathrm{~min}$ at $70^{\circ} \mathrm{C}$. After that $4 \mu \mathrm{l}$ of reverse transcription master mix containing $2 \mu \mathrm{l}$ 10x reaction buffer, 10 units of RNAse Inhibitor, 25 units of reverse transcriptase and $1.625 \mu \mathrm{l}$ of DEPC water were added to each sample. cDNA synthesis was performed at $42^{\circ} \mathrm{C}$ for $1 \mathrm{~h}$ followed by enzyme 
inactivation for $5 \mathrm{~min}$ at $95^{\circ} \mathrm{C}$. Finally, samples were brought to $50 \mu \mathrm{l}$ volume by DEPC water.

\subsubsection{Quantitative real-time PCR}

One $\mu$ l of each ChIP or cDNA sample was used for subsequent quantitative real-time PCR analysis with a final reaction volume of $25 \mu \mathrm{l}$. A PCR reaction was setup as follows: $75 \mathrm{mM}$ Tris- $\mathrm{HCl}$ (pH 8.8), $20 \mathrm{mM}\left(\mathrm{NH}_{4}\right) 2 \mathrm{SO}_{4}, 0.01 \%$ Tween-20, $3 \mathrm{mM} \mathrm{MgCl}$, $200 \mu \mathrm{M}$ dNTPs, 0.5 U/reaction Taq DNA Polymerase, 0.25\% Triton X-100, 1:80,000 SYBR Green I, $300 \mathrm{mM}$ Trehalose and $30 \mathrm{nM}$ primers.

A two-step PCR protocol was used for each primer pair:

$$
2 \min -95^{\circ} \mathrm{C}
$$

$\left(\begin{array}{c}15 \sec -95^{\circ} \mathrm{C} \\ 1 \min -60^{\circ} \mathrm{C}\end{array}\right) \times 40$

The PCR reaction was followed by a melting curve analysis from $60^{\circ} \mathrm{C}$ to $95^{\circ} \mathrm{C}$ with read every $0.5^{\circ} \mathrm{C}$.

ChIP and ChIP input samples were quantified using a standard curve made from ChIP input DNA. ChIP samples were normalized to their corresponding input samples and expressed as "percent input".

Also cDNA samples were quantified using a standard curve made from all cDNA samples. Prior to statistical analysis all qRT-PCR samples were normalized to RPLP0 or $28 \mathrm{~S}$ ribosomal RNA as internal reference genes. The expression levels were determined relative to the control sample and expressed as "relative mRNA expression".

\subsubsection{Microarray analyses}

Total RNA for microarray experiments was isolated as described in 3.2.1 and the Affymetrix DNA microarray analysis was performed at the Transcriptome Analysis Laboratory (TAL), University of Göttingen. The GeneChip $\circledast$ whole transcript labeling was carried out together with Susanne Luthin. Dr. Gabriela Salinas-Riester and Lennart Opitz performed the initial analyses of the microarray data. 
The GeneChip ${ }^{\circledR}$ whole transcript sense targeting labeling assay (Affymetrix) generates amplified and biotinylated sense-strand DNA targets from the entire expressed genome. The GeneChIP® Whole Transcript Labeling Assay is based on the "GeneChIP® Whole Transcript (WT) Sense Target Labeling Assay Manual" (Affymetrix, Santa Clara, USA). This method is well established at the TAL and available as Standard Operating Procedure, SOP Nr. TAL027.1 "GeneChIP® Whole Transcript (WT) Labeling".

\section{$\underline{\text { Target Preparation }}$}

RNA for the Microarray analysis was isolated from cells as described above. First, RNA quality was checked using the Bioanalyzer 2100 (Agilent) to ensure GeneChip $\AA$ whole transcript labeling of high-quality samples. This on-chip gel electrophoresis provides information about size, quantification and quality of RNA. Additionally, to estimate the integrity of RNA samples the RNA integrity number (RIN) is utilized.

First of all, $300 \mathrm{ng}$ of each total RNA sample were reverse transcribed into singlestranded cDNA using T7-(N)6 primers. In order to generate double-stranded cDNA a second strand CDNA synthesis was generated, which in turn was used as a template for in vitro transcription (IVT). The IVT reaction was performed in the presence of T7 RNA polymerase at $37^{\circ} \mathrm{C}$ for $16 \mathrm{~h}$. In addition, the generated antisense cRNA samples were purified and then used for the second cycle of cDNA synthesis. In order to reproducibly fragment single-stranded DNA, dUTP was incorporated in the DNA during the first strand cDNA synthesis. After purifying the single-stranded cDNA samples, they were fragmented using a mix of uracil DNA glycosylase (UDG) and apurinic/apyrimidinic endonuclease 1 (APE 1). These enzymes specifically break the DNA at the dUTP residues not present in normal DNA. Afterwards, the singlestranded DNA samples were labeled by using terminal deoxynucleotidyl transferase (TdT) and biotin-linked Affymetrix® DNA labeling reagent.

\section{$\underline{\text { Target Hybridization }}$}

The target cocktail including fragmented and labeled target DNA as well as hybridization controls was prepared according to the manufacturer instructions for a 169 format array. Upon injection of the specific samples into the arrays, hybridization 
was performed in the GeneChip ${ }^{\circledR}$ hybridization oven at $45^{\circ} \mathrm{C}$ with simultaneous centrifugation at $60 \mathrm{rpm}$ for $17 \mathrm{~h}$.

\section{Washing, staining and scanning of the array}

The GeneChIP® Fluidics Station 450 (Affymetrix, Santa Clara, USA) was used to wash and stain the probe arrays according to the manufacturer's instructions. Subsequently, probe arrays were scanned with the GeneChIP® Scanner $30007 G$ (Affymetrix, Santa Clara, USA).

\section{Data analysis}

The bioinformatics data analysis was performed by Frank Kramer in the Department of Medical Statistics, at the University of Göttingen, using log2 transformation and quantile normalization of expression levels (Bolstad et al., 2003). Background correction was applied according to the manufacturer's advice. In order to determine significant differences of expression levels between the different groups a moderated Student's $t$-test was computed on a gene-by-gene basis using the empirical Bayesian statistics in the 'limma' package (Smyth, 2004). P-values were adjusted for multiple testing using the Benjamini-Hochberg method (Benjamini and Hochberg, 1995) to avoid a high number of false positives and to stay below a false discovery rate of $5 \%$.

\subsubsection{Chromatin immunoprecipitation (ChIP)}

Medium from growing cells was removed from plates and chromatin of the cells were crosslinked by adding 1\% formaldehyde diluted in PBS to the cells. After $15 \mathrm{~min}$ of incubation at room temperature the crosslink was quenched by adding glycine to a final concentration of $125 \mathrm{mM}$. Cells were washed twice with PBS after $5 \mathrm{~min}$ and scraped in $1 \mathrm{ml}$ of Nelson ChIP buffer. All further manipulations were performed on ice or at $4^{\circ} \mathrm{C}$. Scraped cells were cleared from the buffer by centrifugation $(12,000 \mathrm{~g} \mathrm{x}$ $10 \mathrm{~min}$ ) and washed again with $1 \mathrm{ml}$ of Nelson ChIP buffer. After the second centrifugation the pellet was resuspended by softly pipetting up and down in $300 \mu \mathrm{l}$ Weinmann lysis buffer containing protease, phosphatase and deubiquitinase inhibitors and incubated at $4^{\circ} \mathrm{C}$ on a wheel for $15 \mathrm{~min}$. The sonication process was done in a Bioruptor ( $3 \times 10 \mathrm{~min})$ at " $\mathrm{H}$ " setting with $10 \mathrm{~s}$ breaks between $10 \mathrm{~s}$ pulses. The soluble chromatin was cleared from the debris by centrifugation $(12000 \mathrm{~g} \times 10$ 
min at $8^{\circ} \mathrm{C}$ ) and pre-cleared with $100 \mu \mathrm{l}$ of $50 \%$ slurry Sepharose beads for $1 \mathrm{~h}$. After the pre-clearing the chromatin was centrifuged, aliquoted, frozen in liquid nitrogen and stored at $-80^{\circ} \mathrm{C}$. For the analysis $300 \mu \mathrm{l}$ of chromatin were diluted up to $1 \mathrm{ml}$ with ChIP dilution buffer and incubated overnight with the indicated amount of antibodies. Immunoglobulin bound complexes were precipitated by adding $30 \mu \mathrm{l}$ of $50 \%$ slurry Protein-A or Protein-G Sepharose and incubation for another $2 \mathrm{~h}$. After incubation the samples were centrifuged $(2,000 \mathrm{~g} \times 2 \mathrm{~min})$. The beads were washed with ice-cold low-salt wash buffer two times, LiCl wash buffer two times and TE buffer two times and the crosslink was reversed by adding $10 \%$ slurry Chelex with subsequent heating to $95^{\circ} \mathrm{C}$ for $10 \mathrm{~min}$. The proteins in the sample were eliminated by adding $40 \mu \mathrm{g}$ of Proteinase $\mathrm{K}$ and incubating for $30 \mathrm{~min}$ at $55^{\circ} \mathrm{C}$. The samples were centrifuged $(12,000 \mathrm{~g} \times 1 \mathrm{~min})$ and the supernatant was analysed by quantitative real-time PCR. The background binding was determined by performing a ChIP with a non-specific IgG antibody. To prepare the ChIP inputs $10 \mu \mathrm{l}$ (10\% relative to ChIPs) of chromatin extracts were precipitated by adding $100 \% \mathrm{EtOH}$ and $1 \mu \mathrm{l}$ of Pink precipitant (5 $\mathrm{mg} / \mathrm{ml}$ ) and incubating overnight at $-20^{\circ} \mathrm{C}$. The pellets were washed twice with $70 \%$ $\mathrm{EtOH}$, dried and processed with Chelex addition as described above for ChIP samples. The ChIP samples were normalized to input DNA samples, and displayed as "\% of input".

\subsubsection{Chromatin immunoprecipitation-sequencing (ChIP-Seq)}

ChIP-seq combines chromatin immunoprecipitation with DNA sequencing to identify binding sites of DNA-associated proteins. The chromatin for the ChIP-seq was isolated and immunoprecipitated from cells as described above with the exception that after the last washing step of the beads, the DNA was isolated using phenol chloroform extraction.

\section{DNA isolation using phenol chloroform extraction}

For this purpose $50 \mu \mathrm{l}$ of RNAse A (10 $\mu$ g) diluted in $10 \mathrm{mM}$ Tris, pH 8 was added to the washed chromatin-bound beads as well as the input samples and incubated 30 min at $37^{\circ} \mathrm{C}$. Afterwards $50 \mu \mathrm{l}$ of Wienmann buffer and $20 \mu \mathrm{g}$ of Proteinase $\mathrm{K}$ were added and the samples were incubated overnight at $65^{\circ} \mathrm{C}$ with a subsequent centrifugation step at $2000 \mathrm{~g}$ for $2 \mathrm{~min}$ at RT. The supernatant was collected, and the 
beads were rinsed again with $100 \mu \mathrm{l}$ of Tris $10 \mathrm{mM} \mathrm{pH}$ 8. The samples were centrifuged and the supernatant was added to the first one. For extraction $10 \mu \mathrm{l}$ of 8 $\mathrm{M} \mathrm{LiCl}, 4 \mu \mathrm{l}$ co-precipitant and $200 \mu \mathrm{l}$ phenol/chloroform/isoamyl alcohol (25:24:1) were added, samples were vortexed for $30 \mathrm{~s}$ and centrifuged for $2 \mathrm{~min}$ at full speed. The aqueous phase was collected and the phenol phase was back extracted with $200 \mu \mathrm{l} 10 \mathrm{mM} \mathrm{Tris} / \mathrm{HCl}, \mathrm{pH} 8$ and $400 \mathrm{mM} \mathrm{LiCl}$. After vortexing and centrifugation, the second aqueous phase was given to the first one and precipitation was performed by supplementation of $100 \% \mathrm{EtOH}$ and incubation for $2 \mathrm{~h}$ at $-80^{\circ} \mathrm{C}$. After that, samples were centrifuged at maximal speed for $30 \mathrm{~min}\left(4^{\circ} \mathrm{C}\right)$ pellets were washed with $70 \%$ ethanol, dried on vacuum concentrator and re-dissolved in $40 \mu \mathrm{l}$ of water.

$5 \mu \mathrm{l}$ of the DNA was used for QRT-PCR to confirm the efficient chromatin immunoprecipitation and $35 \mu \mathrm{l}$ for ChIP-seq. DNA concentration was measured using a Qubit dsDNA HS assay on a Qubitß 2.0 Fluorometer.

\section{Shearing check}

In order to analyze the efficiency of the sonification a shearing check was performed. Therefore $10 \mu \mathrm{l}$ of sheared chromatin were used for phenol chloroform extraction. After resuspension of the DNA in $15 \mu \mathrm{l} \mathrm{Tris} / \mathrm{HCl} 10 \mathrm{mM}, \mathrm{pH} 8,100 \mu \mathrm{g} / \mathrm{ml}$ RNAse A was added and the mixture was incubated $1 \mathrm{~h}$ at $37^{\circ} \mathrm{C}$ at $700 \mathrm{rpm}$. The DNA was run on a $1.5 \%$ agarose gel and when the shearing was sufficient a smear from about 150 to $1 \mathrm{~kb}$ with a maximum around $300-400$ bp was observed.

\section{Library preparation}

The cDNA library preparation was performed by Magali Hennion at the DZNE, the German Centre for Neurodegenerative Diseases within the Helmholtz Association. The ChIP-sequencing itself was carried out at the Transcriptome Analysis Laboratory (TAL), University of Göttingen.

To start the library at least $10 \mathrm{ng}$ of DNA is necessary. First, sonication process was perfomed on NGS Bioruptor by 10 cycles with $30 \mathrm{~s}$ on and $30 \mathrm{~s}$ off. Afterwards, DNA was checked for size, quantity and quality using Bioanalyzer DNA High sensitivity kit on a Bioanalyzer 2100 (Agilent). To perform end repair a reaction was setup as follows: $5 \mu \mathrm{l} \mathrm{T4}$ DNA ligase buffer with $10 \mathrm{mM}$ ATP, $2 \mu \mathrm{l} 10 \mathrm{mM}$ dNTP mix, $1 \mu \mathrm{l} \mathrm{T} 4$ 
DNA polymerase $(3 \mathrm{U}), 1 \mu \mathrm{l}$ Klenow DNA polymerase $(1 \mathrm{U}), 1 \mu \mathrm{l}$ T4 PNK $(10 \mathrm{U})$ and filled up with water to $50 \mu \mathrm{l}$ total volume. After the incubation at $20^{\circ} \mathrm{C}$ for $30 \mathrm{~min}, 2 \mu \mathrm{l}$ of colorless coprecipitant and $50 \mu \mathrm{L}$ SureClean were added and the samples were vortexed and incubated for $10 \mathrm{~min}$ at RT. In addition, the samples were centrifuged $(15,000 \mathrm{~g} \times 20 \mathrm{~min})$ and the pellets were washed twice with $70 \% \mathrm{EtOH}$, dried and resuspended in $10 \mathrm{mM}$ Tris $/ \mathrm{HCl}, \mathrm{pH}$ 8. Adding Adenosine nucleotides to the 3 '-end of the DNA fragments occurs in a similar way described for end repair, except the composition of the master mix which contains: $5 \mu \mathrm{l}$ 10X Klenow buffer, $10 \mu \mathrm{l} 1 \mathrm{mM}$ dATP, $1 \mu \mathrm{l} \mathrm{Klenow}$ fragment ( 3 ' to $5^{\prime}$ exo-) $(5 \mathrm{U}$ ) and the subsequent $30 \mathrm{~min}$ incubation was performed at $37^{\circ} \mathrm{C}$. To ligate adapters to the DNA fragments $15 \mu \mathrm{l}$ of 2x T4 Quick ligase buffer, 16,7 nM Adapter oligo mix and $3 \mu \mathrm{l}$ Quick ligase were directly added to the sample (final volume $30 \mu \mathrm{l}$ ) and incubated for $30 \mathrm{~min}$ at $25^{\circ} \mathrm{C}$. Again precipitation and washing steps were carried out as described. In order to select and collect DNA with a well-suited size, Pippin Prep size (Sage Science) selection was performed. For this purpose samples were run on a $2 \%$ agarose cassette and selected from $200 \mathrm{bp}$ to $300 \mathrm{bp}$.

Five $\mu$ l of DNA sample was used for subsequent quantitative real-time PCR analysis with a final reaction volume of $15 \mu \mathrm{l}$. A PCR reaction was setup as follows: $0.3 \mu \mathrm{l}$ Primers PCR IndX + PCR $1.025 \mu \mathrm{M}$ (500 nM final), $3 \mu \mathrm{l}$ 5X HF buffer, 0,02 $\mu \mathrm{l}$ SybrGreen 1:100 and 0,15 $\mu \mathrm{l}$ Phusion polymerase $(2 \mathrm{U} / \mu \mathrm{l})$.

The reaction was incubated under the following conditions:

$$
1 \min -98^{\circ} \mathrm{C}
$$

$\left(\begin{array}{l}10 \mathrm{sec}-98^{\circ} \mathrm{C} \\ 30 \mathrm{sec}-65^{\circ} \mathrm{C} \\ 30 \mathrm{sec}-72^{\circ} \mathrm{C}\end{array}\right) \times 30$

This quantitative PCR was used to determine the minimum number of PCR cycles needed to recover enough DNA for sequencing. A PCR to amplify $20 \mu$ of DNA was carried out with $0.8 \mu \mathrm{l}$ Primers PCR IndX + PCR $1.025 \mu \mathrm{M}$ (500 nM final), $8 \mu \mathrm{l} 5 \mathrm{X}$ HF buffer, $0.8 \mu \mathrm{l}$ dNTP $10 \mathrm{mM}$ and $0.4 \mu \mathrm{l}$ Phusion polymerase $(2 \mathrm{U} / \mu \mathrm{l})$ and filled up with water to $40 \mu \mathrm{l}$. IndX means that different indexes were used for multiplexing. 


$$
1 \min -98^{\circ} \mathrm{C}
$$

$\left(\begin{array}{c}10 \mathrm{sec}-98^{\circ} \mathrm{C} \\ 30 \mathrm{sec}-65^{\circ} \mathrm{C} \\ 30 \mathrm{sec}-72^{\circ} \mathrm{C}\end{array}\right) \times<18$ (adjusted according to previous $\mathrm{q}-\mathrm{RT}$-PCR)

Again precipitation and washing steps were carried out as described above and the amplified DNA concentration was measured by using the Qubit dsDNA HS assay and the size was checked using a Bioanalyzer. The enrichment of known positive sites was checked by qRT-PCR.

The samples were pooled (4-6 samples/lane) and sequenced using Illumina HiSeq 2000 following the manufacturer instructions.

\section{Data analysis}

The H2Bub1 ChIP libraries from two control and two ATXN7L3 KD samples were sequenced using Illumina HiSeq 2000. One input sample from each condition was also sequenced. Raw data were converted into fastq files. 30 to 39 million reads per sample were obtained. Data for $\mathrm{H} 3 \mathrm{~K} 4 \mathrm{me} 1, \mathrm{H} 3 \mathrm{~K} 4 \mathrm{me} 3$ and $\mathrm{H} 3 \mathrm{~K} 9 / 14 \mathrm{ac}$ were obtained from the European Nucleotide Archive (http://www.ebi.ac.uk/ena/; study accession number SRP007976). The quality of the runs was checked with FastQC (www.bioinformatics.babraham.ac.uk/projects/fastqc/). The reads were mapped against the reference genome hg19 using Bowtie (http://bowtie-bio.sourceforge.net) with default parameters and wig files were generated with $10 \mathrm{bp}$ windows using MACS (Zhang et al., 2008b). After combining the duplicates, metagene profiles for H2Bub1 were plotted using CEAS (Shin et al., 2009). The genes were sorted into 4 quartiles according to gene expression level in control MCF10A cells (data obtained by our microarray). The same was done including input subtraction in the wig files and the resulting profiles were very similar. 


\subsection{Protein biochemistry}

\subsubsection{SDS-PAGE}

Upon SDS-PAGE, the proteins were separated via sodium dodecylsulfate polyacrylamide gel electrophoresis (SDS-PAGE) (Laemmli, 1970). Cells were lysed in RIPA buffer containing $1 \mathrm{mM}$ Pefabloc, $1 \mathrm{ng} / \mu \mathrm{l}$ Aprotinin/Leupeptin, $10 \mathrm{mM}$ BGP and $1 \mathrm{mM}$ NEM. In order to shear genomic DNA, samples were sonicated for $10 \mathrm{~s}$ at $10 \%$ power using a Bandelin Sonoplus sonicator. Protein samples were boiled in Laemmli buffer for $5 \mathrm{~min}$ and then subjected to SDS-PAGE. The composition of stacking and resolving gel are described in section 2.8. Polyacrylamide gels were run in SDS running buffer at $25 \mathrm{~mA}$.

\subsubsection{Western blot analysis}

Subsequently, upon electrophoresis proteins were detected by Western blot analysis (Towbin et al., 1979) using target protein specific antibodies. Separated proteins were transferred at $25 \mathrm{~V}$ to PVDF membranes using transfer buffer for $1.5-2 \mathrm{~h}$, depending on the size of the protein. The membranes were incubated for $30 \mathrm{~min}$ in PBS-T and 5\% (w/v) dry milk to block non-specific antibody binding. Afterwards the membranes were incubated overnight at $4^{\circ} \mathrm{C}$ in the same blocking buffer containing the respective primary antibodies, diluted as described in the antibody table (2.6.3.1). After washing three times with PBS-T, the membranes were incubated for $1 \mathrm{~h}$ with the corresponding horseradish peroxidase-conjugated anti-mouse IgG or anti-rabbit $\lg \mathrm{G}$ secondary antibodies at a dilution of 1:10,000. After washing three times with PBS-T, HRP signals were detected using enhanced chemoluminescence and exposed to X-ray films.

\subsubsection{In vitro deubiquitination assay}

Protein extracts from normal MCF10A cells (substrate) were mixed with extracts from cells where RNF20 and RNF40 were depleted with siRNA to minimize the level of H2Bub1 (effector). Cells were washed with PBS and scraped in Pagano buffer. After mixing cells were sonicated with tip sonicator for $15 \mathrm{~s}$ and centrifuged at full speed to remove the cell debris. One volume of target supernatant was mixed with one volume of effector supernatant and two volumes of deubiquitination buffer. The reaction was 
performed with shaking at $37^{\circ} \mathrm{C}$ for 15 min and stopped by adding Laemmli buffer and heating to $95^{\circ} \mathrm{C}$ for $5 \mathrm{~min}$. The reaction products were analyzed by SDS-PAGE Western blot.

\subsubsection{Immunohistochemistry on paraffin sections}

Paraffin-embedded sections were de-paraffinized and rehydrated according to standard procedures. Briefly, after incubating in xylene for $20 \mathrm{~min}$, the sections were rehydrated in a 100\%, 90\% and $70 \% \mathrm{EtOH}$ series before washing with PBS. Proteins were then unmasked by boiling slides in $10 \mathrm{mM}$ citric acid/sodiumphosphate. After washing with PBS, sections were quenched for endogenous peroxidase activity with $3 \%$ hydrogen peroxide in PBS for $45 \mathrm{~min}$ at RT and then blocked using 5\% FCS diluted in PBS for $1 \mathrm{~h}$ at RT. The respective antibody was diluted 1:10 in PBS containing 5\% FCS, applied and incubated overnight at $4^{\circ} \mathrm{C}$ in a humid chamber. Sections were washed using PBS before adding the biotinylated secondary antibody 1:200 diluted in PBS and incubated for $1 \mathrm{~h}$ at RT. PBS washings were followed by Avidin-Peroxidase incubation 1:1,000 diluted in PBS for 45 min. Staining signals were detected using diaminobenzidine substrate. Hematoxyline (Mayer's hemalaun solution) was used for counterstaining. Histological slides were digitized with a ScanScope XT (Aperio) at 400x magnification. Color intensity quantification was achieved with ImageJ software. 


\section{Results}

H2B monoubiquitination (H2Bub1) is implicated in various cellular processes including gene expression, DNA damage repair and nucleosomal exchange. H2Bub1 is associated with the transcribed region of active genes, and transcription is essential for maintaining global levels of H2Bub1. Misregulation of the H2Bub1modifying machinery is tightly linked with different types of cancer. The main focus of this study was to uncover mechanisms of H2Bub1 regulation upon stress-inducing factors.

\subsection{H2B ubiquitinating enzymes affect cellular responses}

To assess the function of $\mathrm{H} 2 \mathrm{~B}$ monoubiquitination the role of ubiquitin ligases involved was examined within a cellular context. Both RNF20 and RNF40 form an obligatory heterodimer in vivo. Consistent with its role within the major E3 ubiquitin ligase complex for histone H2B, RNF20 knockdown leads to a loss of global H2Bub1. Conflicting reports were published on the role of RNF40 in H2B monoubiquitination (Kim et al., 2005; Weake and Workman, 2008; Zhu et al., 2005). Our own working group could show that knockdown of either RNF20 or RNF40 using multiple siRNAs similarly decreased global levels of H2Bub1 (Pirngruber et al., 2009a). The main way to dissect H2Bub1 function experimentally is to deplete or overexpress one of its ubiquitinating enzymes and to analyze the resulting biological effects. Therefore, the siRNA-knockdown system was established for different cancer cell lines. The main focus was on MCF10A, a non-transformed breast epithelial cell line and U2OS, a human osteosarcoma cell line.

\subsubsection{Effects of RNF40 depletion on proliferation of breast cancer and osteosarcoma cells}

The colony formation assay is an in vitro cell survival assay which is based on the fact that a single cell can grow into a colony. The assay detects all cells which are able to produce progeny under specific experimental conditions. Therefore, it is the method of choice in this study to determine the effects of RNF40 depletion via siRNAmediated knockdown on the proliferative capacity of MCF10A and U2OS cells. 
Figure 5A shows the outcome of the clonogenic assay upon RNF40 knockdown. Different numbers of cells were seeded one day after transfection and analyzed seven days later. RNF40 depletion showed an anti-proliferative effect on both MCF10A and U2OS cells in this study, as depicted by a lower number of crystal violet-stained colonies compared to control cells. Western blot results in figure $5 \mathrm{~B}$ showed that the RNF40 protein level is still reduced eight days after transfection.

A

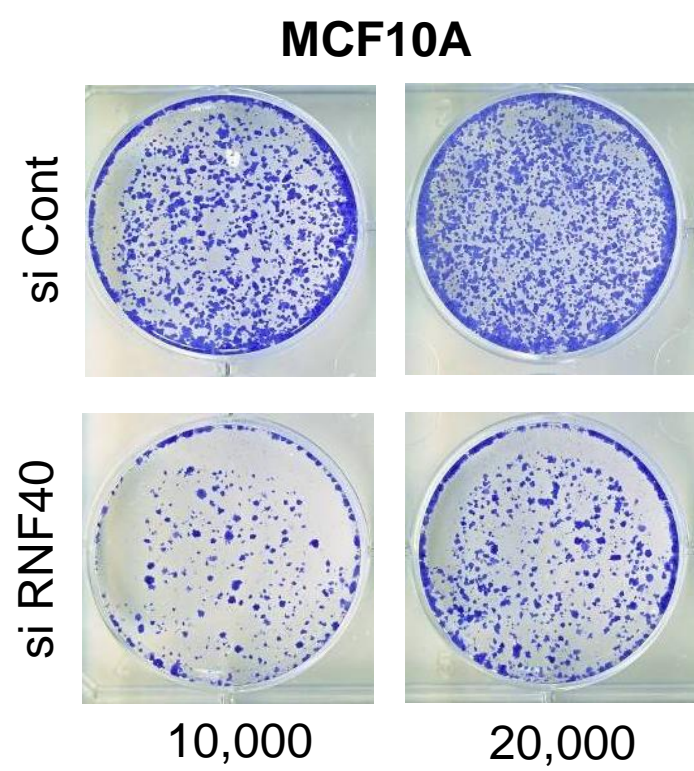

B

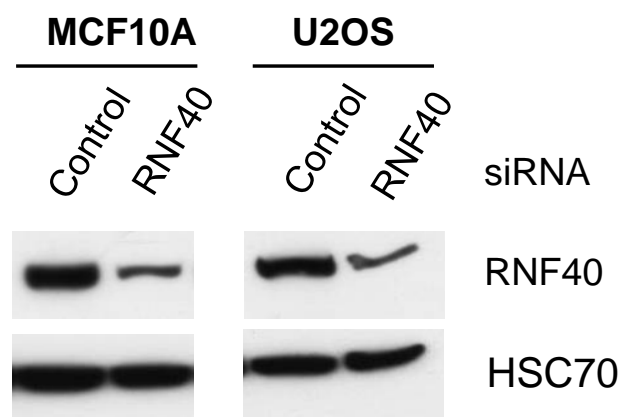

U2OS
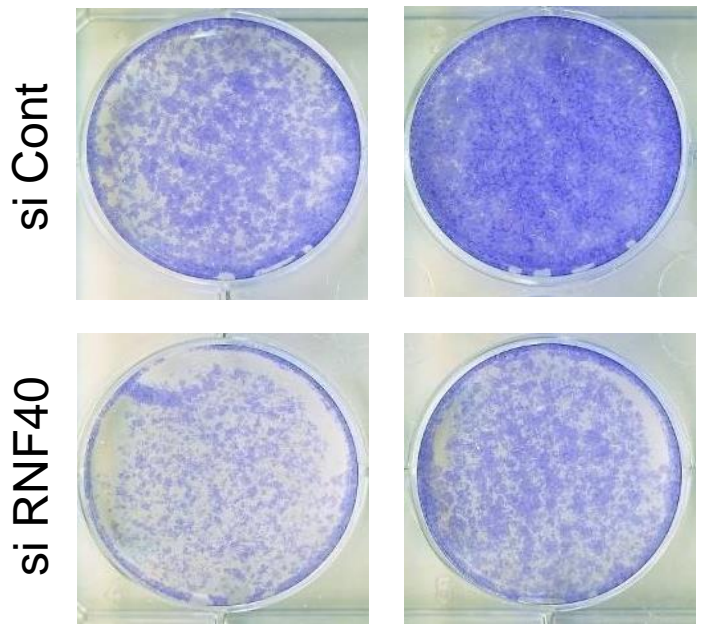

5,000

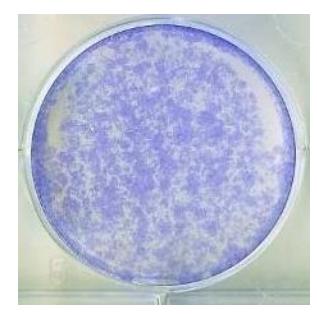

10,000

Fig. 5: siRNA-mediated knockdown of RNF40 increased colony formation in MCF10A cells and U2OS cells in vitro. (A) One day after reverse-transfection with $30 \mathrm{pmol}$ control or RNF40 siRNA, transfected MCF10A cells were re-plated into 6 -well plates with a density of 10,000 or 20,000 cells per well and U2OS cells with a density of 5,000 or 10,000 cells per well. Colonies were fixed with $70 \%$ methanol after eight days visualized by staining with $0.1 \%$ crystal violet. Representative images of the colonies formed in two independent experiments with both cell lines are shown. (B) In parallel, efficient knockdown of RNF40 eight days post transfection was confirmed by Western blot analysis a using RNF40-specific antibody and equal loading was controlled by using HSC70 antibody. 


\subsubsection{RNF20 and RNF40 depletion leads to a cell cycle arrest}

Next we analyzed whether or not the anti-proliferative effect of RNF20 and RNF40 knockdown observed above may be due to the negative effects of these ligases for cell cycle progression. As shown in figure 6A, siRNA-mediated knockdown of RNF40 in MCF10A cells increased the fraction of cells in $\mathrm{G} 1$ phase $72 \mathrm{~h}$ post transfection (56\% in RNF40 vs. $43 \%$ in control-siRNA-transfected cells) while the percentage of cells in S phase (18\% in RNF40 compared to $29 \%$ in control-siRNA-transfected cells) was decreased. RNF20 and RNF40 form a heterodimer complex and depletion of RNF20 effectively decreases amount of RNF40 but not vice versa which shows the requirement of RNF20 for RNF40 protein levels (Kim et al., 2009). Similarly to the cell cycle profile of RNF40 knockdown cells, RNF20 depletion showed a G1 cell cycle arrest with decreased cells in $S$ phase. These observations suggest that siRNAmediated knockdown of RNF20 and RNF40 inhibits $S$ phase entry and thus increases the G1 fraction of MCF10A cells. Decreased RNF20 and RNF40 levels seem to be sufficient to affect cellular metabolism and cause a G1 arrest or block $S$ phase entry in MCF10A cells. Figure 6B shows that siRNAs targeting RNF20 and RNF40 efficiently reduced the expression level of RNF40 protein compared to control SiRNA. 
A

\section{Cont siRNA}

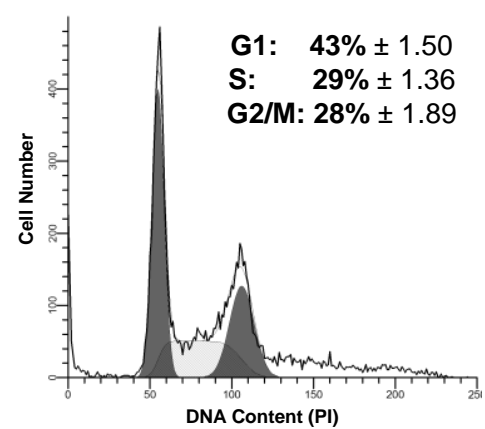

RNF20 siRNA

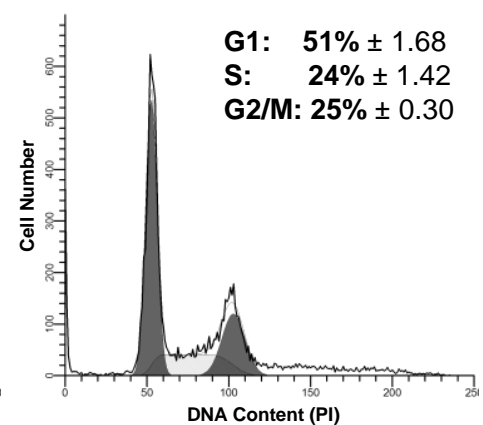

RNF40 SiRNA

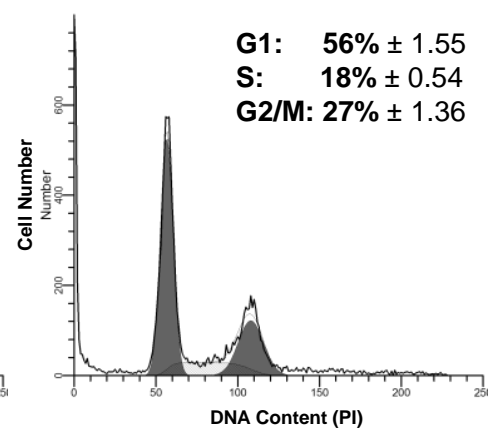

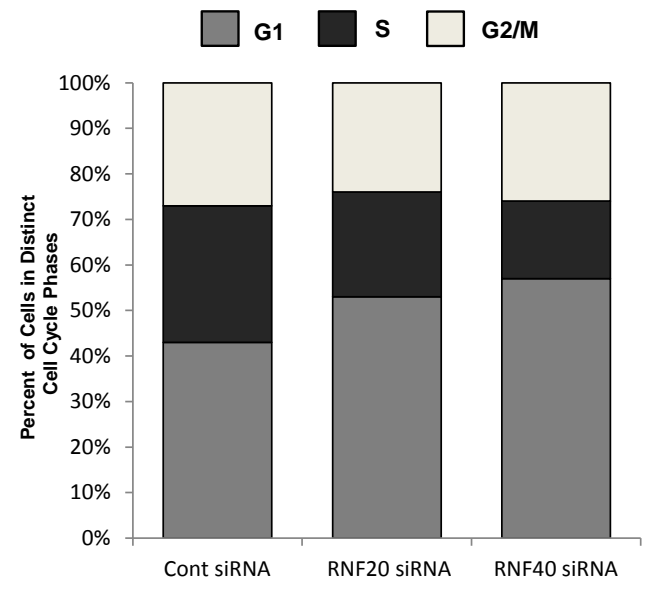

B

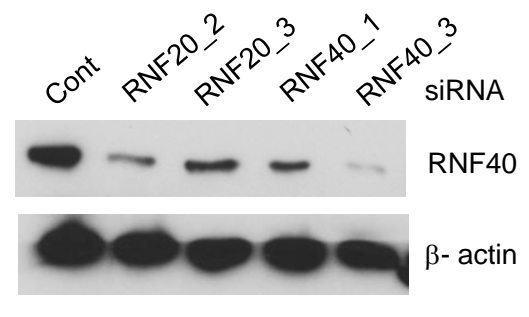

Fig. 6: Knockdown of RNF20 and RNF40 increases the percentage of MCF10A cells in G1 phase. (A) Cell cycle distribution of RNF20, RNF40 or control siRNA-transfected MCF10A cells was determined after $72 \mathrm{~h}$ by staining with propidium iodide $(\mathrm{PI})$ and analyzed by flow cytometry. Distribution of cells in distinct cell cycle phases was determined and graphically displayed using ModFIT cell cycle analysis software. The respective siRNAs are indicated above the ModFIT graphs. FACS profiles show $\mathrm{G} 1$ cell cycle arrest. In each case, 10,000 cells were analyzed. $\pm S D, n=4$. The percentage of cells in distinct cell cycle phases is shown as a bar graph underneath. (B) Two different siRNA were used to deplete RNF20 and RNF40. The efficient knockdown was confirmed by Western blot analysis using a RNF40-specific antibody. $\beta$ - actin served as loading control.

\subsubsection{Effects of RNF40 overexpression on cell proliferation}

In order to determine the proliferation capacity in cells which overexpress RNF40 a stable cell line with inducible Flag-RNF40 expression was utilized. The benefit of using U2OS cells expressing Tet-R (Monroe et al., 2003) is the doxycyclineinducibility of Flag-RNF40 overexpression which helps to avoid negative selection. An U2OS-Tet-R cell line was used as a control to exclude possible side effects of the doxycycline treatment. Both U2OS cell lines were treated with doxycycline and RNF40 expression was examined by Western blot (Fig. 7A). Using a RNF40 antibody 
to analyze RNF40 overexpression showed an increased level one and two days post doxycycline treatment compared to uninduced U2OS cells. However, the amount of H2Bub1 only showed also a slight increase compared to the increased expression level of RNF40. Since RNF20 and RNF40 act in a complex, RNF40 overexpression alone may not be sufficient to induce H2Bub1 levels to a greater degree.

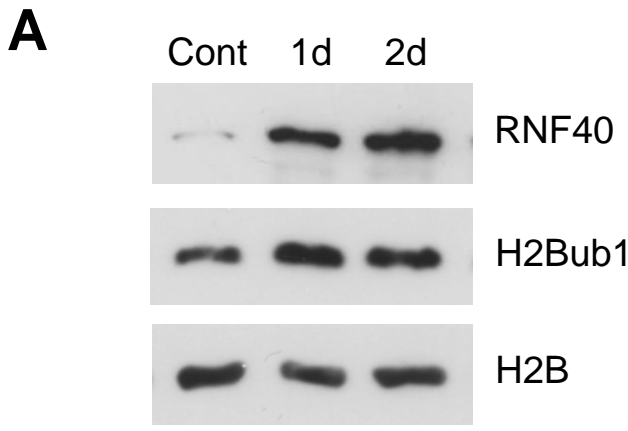

B

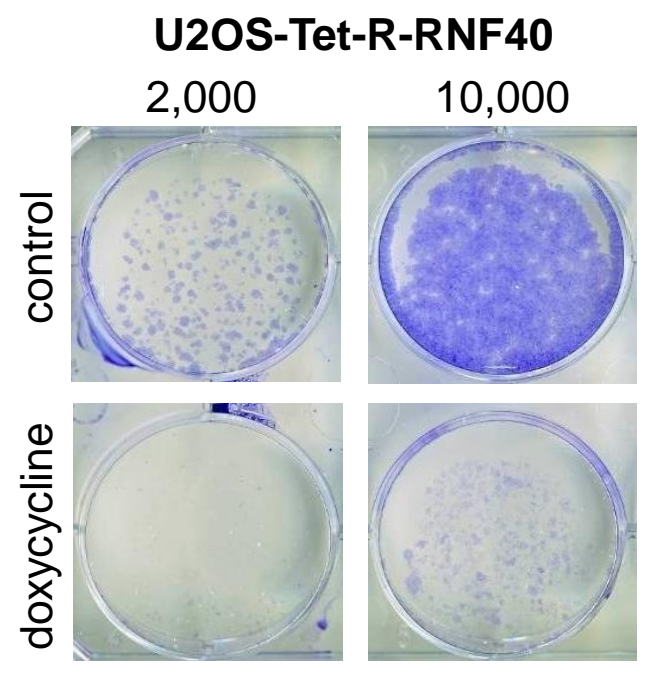

\section{Control cell line}

U2OS-Tet-R

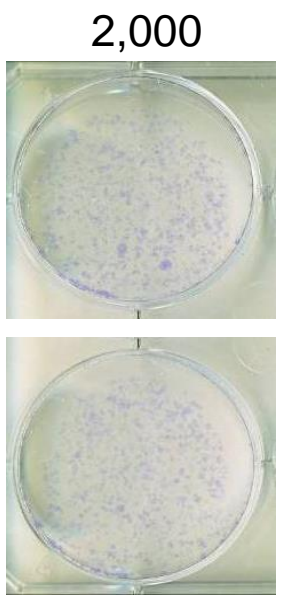

10,000

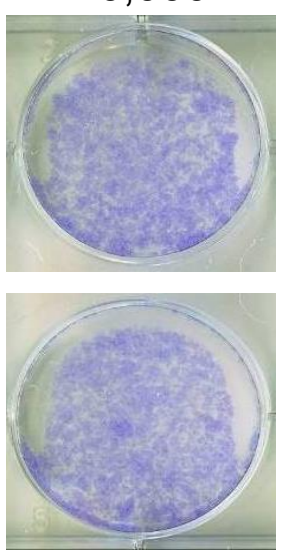

Fig. 7: Ectopic expression of RNF40 inhibits tumor cell growth. (A) Protein extracts were collected after $24 \mathrm{~h}$ and $48 \mathrm{~h}$ and were analyzed by Western blotting using antibodies to RNF40 and H2Bub1 to evaluate the induction of RNF40 and the concomitant increased H2Bub1 level. Analysis of H2B served as a loading control. (B) Effect of RNF40 overexpression on tumor cell growth in cultured human osteosarcoma cells, as determined by the colony formation assay. 2,000 and 5,000 U2OS cells expressing the RNF40-Tet-R were grown under Blasticidin $(5 \mathrm{mg} / \mathrm{ml})$ and Zeocin $(500 \mathrm{mg} / \mathrm{l})$ selection conditions and were seeded in a 6-well plate. The following day, cells were treated with doxycycline to induce RNF40 expression. The medium was changed every second day and after eight days colonies were fixed with $70 \%$ methanol and stained with $0.1 \%$ crystal violet. Representative samples from an experiment with $n=2$ are shown. 
Analysis of the consequence of Flag-RNF40 overexpression eight days post doxycycline induction in U2OS cells by the colony formation assay (Fig. 7B) revealed a striking difference between RNF40 overexpressing and control U2OS cells. While control Tet-R cells showed a very similar proliferative capacity compared to doxycycline treated Tet-R cells, the Tet-R-RNF40 cells completely failed to form colonies upon doxycycline treatment to induce RNF40 expression (Fig. 7B).

\subsubsection{RNF40 overexpression induces a G2/M arrest in osteosarcoma cells}

In order to determine whether the anti-proliferative effect of doxycycline-induced RNF40 overexpression in U2OS-Tet-R-RNF40 cells involved alterations in cell cycle progression, flow cytometry analysis using propidium iodide (PI) was performed. Under normal conditions (control), 51\% of the U2OS-Tet-R-RNF40 cells were in G1, $40 \%$ in $S$ and $9 \%$ in G2/M phase. RNF40 overexpression increases the fraction of Tet-R-RNF40 cells in G2/M phase (16\% vs. 9\% in control cells) (Fig. 8). These data indicate that the negative effect of RNF40 overexpression on the colony formation capacity of Tet-R-RNF40 U2OS cells is due to an induced G2/M cell cycle arrest.

All in all, these results showed anti-proliferative effects of siRNA-mediated knockdown of RNF20 or RNF40 on MCF10A and U2OS cell cycle progression as well as inducible RNF40 overexpression in U2OS Tet-R-RNF40 cells. 
Control

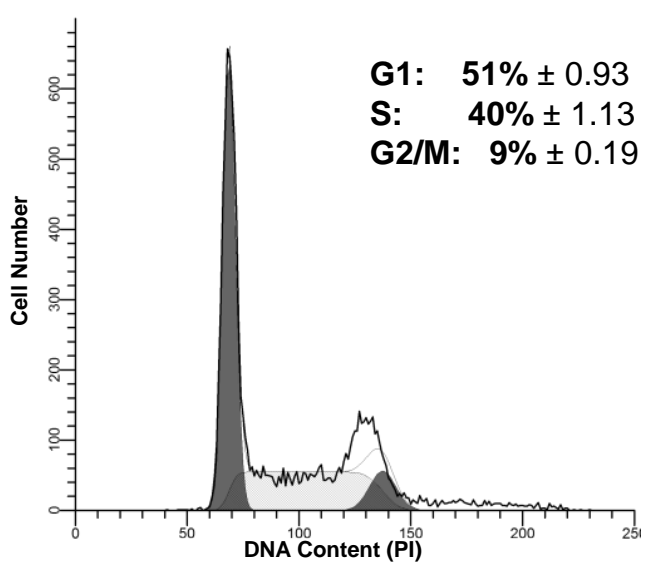

RNF40 overexpression

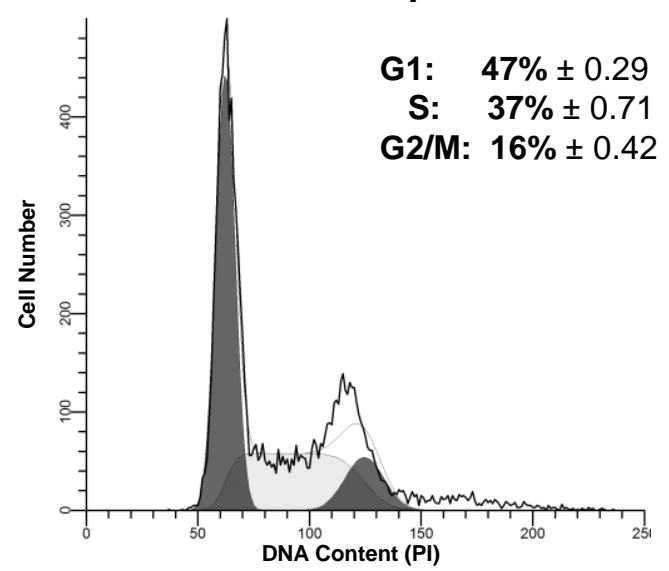

G1 $\mathrm{s}$ G2/M

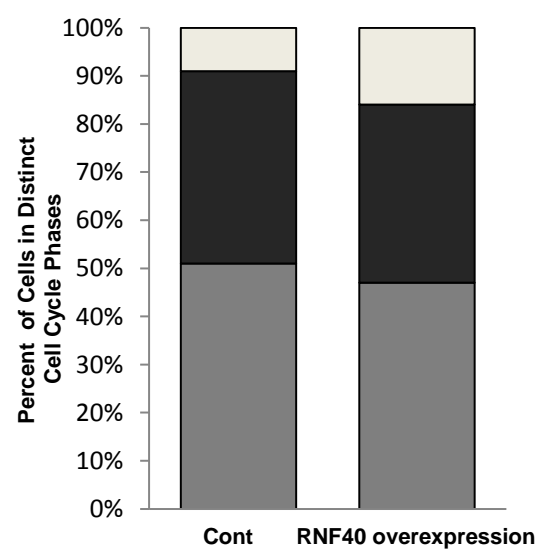

Fig. 8: RNF40 overexpression increases the percentage of U2OS-Tet-RNF40 cells in G2/M phase of the cell cycle. Cell cycle distribution analyses of vector- and RNF40-transfected inducible U2OS-Tet-RNF40 cells. Cells were analyzed $48 \mathrm{~h}$ after doxycycline treatment to induce RNF40 expression. For analysis of cell cycle distribution, U2OS-Tet-RNF40 cells were stained with propidium iodide (PI) and analyzed by flow cytometry using a FACScan. Distribution of cells in distinct cell cycle phases was determined and graphically displayed using ModFIT cell cycle analysis software. \pm SD, $\mathrm{n}=3$. The percentage of cells in distinct cell cycle phases is shown as a bar graph underneath.

\subsubsection{RNF20 and RNF40 similarly suppress migratory potential}

Based on results of the clonogenic assay, the flow cytometric analysis and the importance of cell migration for metastasis, we also investigated the effects of RNF20 and RNF40 knockdown on the migratory potential of a normal mammary epithelial cell line. MCF10A cells are usually nonmigratory (Fig. 9 -EGF), but can be stimulated to migrate by addition of epidermal growth factor (EGF) (Fig. $9+$ EGF). Consistent with a role for H2Bub1 in suppressing metastasis and with the previous study for 
RNF20 (Shema et al., 2008), siRNA-mediated knockdown of RNF20 or RNF40 resulted in a strongly increased EGF-induced cell migration compared to control siRNA in MCF10A cells. Since enhanced cell migration is a distinctive feature of advanced tumors, this finding suggests that decreased RNF20/RNF40 expression may potentially facilitate cancer progression.
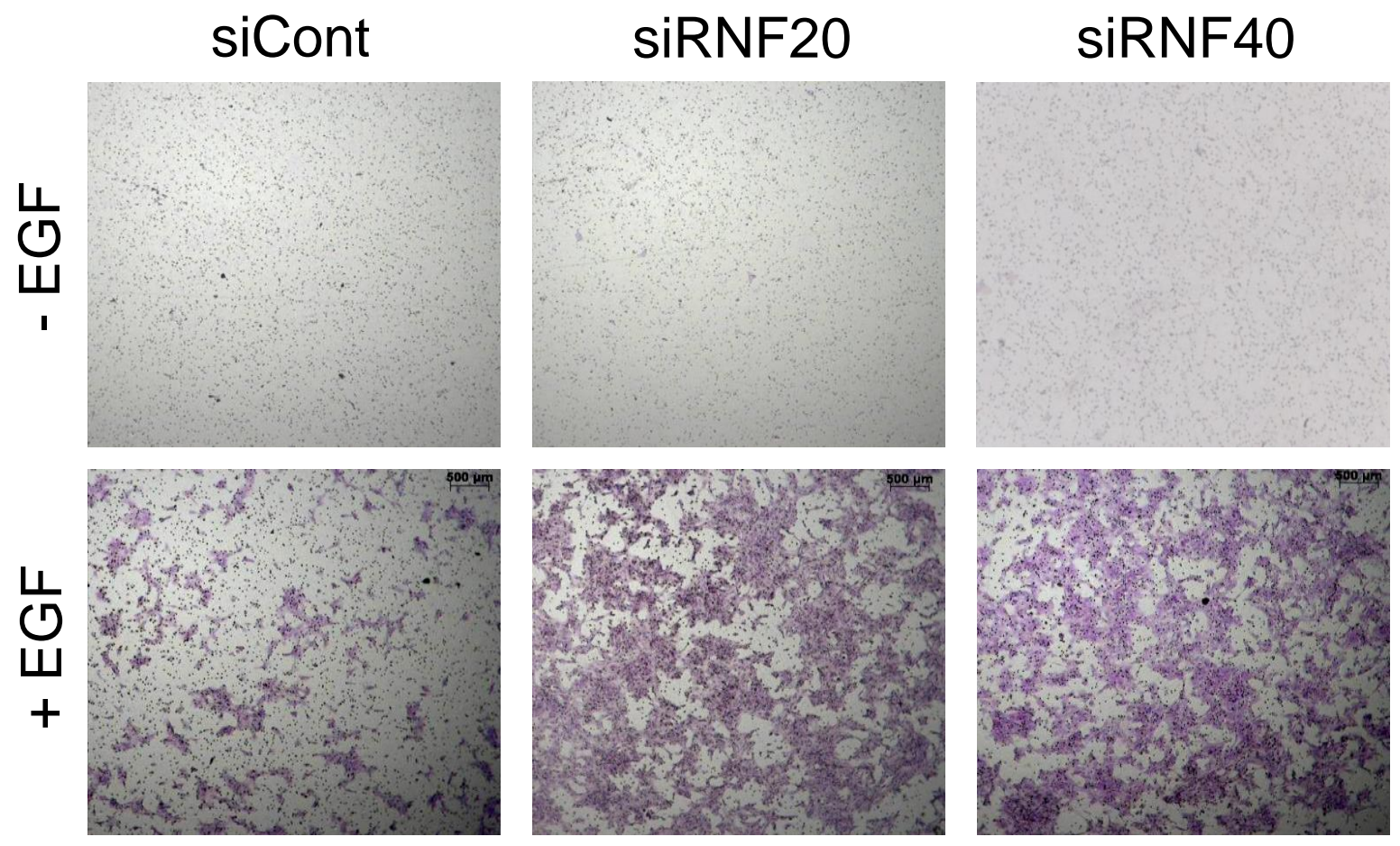

Fig. 9: Cell migration assay after RNF20 or RNF40 knockdown. Control as well as RNF20 or RNF40 siRNA transfected MCF10A cells were seeded onto cell culture inserts and allowed to migrate for $48 \mathrm{~h}$ in either presence or absence of EGF. Representative images from an experiment with $n=2$ are shown, bar $1 / 4500 \mathrm{~mm}$.

\subsubsection{Messenger RNA expression profiling of RNF20 and RNF40-regulated genes}

As mentioned previously H2Bub1 was proposed to be required for transcriptional elongation and its depletion can result in altered gene expression. In order to address the possible involvement of the E3 ubiquitin ligases RNF20 and RNF40 in the regulation of mammalian gene expression, we performed expression microarray analysis on MCF10A cells after siRNA-mediated knockdown of endogenous RNF20 and RNF40. In order to ensure that off-target effects do not create misleading results, we used two independent siRNAs against RNF40 to produce the same results. 
Shema et al. (2008) generated microarray data from RNF20 depleted HeLa cells. Since no study so far investigated the role of RNF40 in the regulation of the mammalian gene expression (Fig. 10A), we performed expression microarray analysis for RNF40. In order to investigate whether the same set of genes were affected in MCF10A cells compared to the published results (Shema et al., 2008) and to check the overlap of RNF20 and RNF40 regulated genes we used siRNA against both RNF20 and RNF40. Efficient knockdown of RNF20 and RNF40 and the subsequent near complete ablation of H2Bub1 was achieved as visualized by Western blotting with H2Bub1-specific monoclonal antibodies, whereas the HSC70 protein level analyzed as a control was not significantly affected (Fig. 10B).

The results of the expression microarray analysis are shown in figure $10 \mathrm{~A}$, which portrays the ratio between the expression level of each gene following RNF20 and RNF40 knockdown relative to their levels in cells transfected with control siRNAs. The mRNA expression profiling was accomplished by DNA microarray analysis for a total of 11,223 genes. The heatmap shows the fold-changes (FCs) in the expression of 525 genes which are significantly $(q<0.05)$ regulated by either RNF20 or RNF40 depletion $(\mathrm{FC}<-\log 2(1.5)$ or $\mathrm{FC}>\log 2(1.5))$.

Previous genome-wide analysis revealed a general positive correlation between gene expression levels and steady-state H2Bub1 levels (Minsky et al., 2008). Nevertheless, the majority of transcribed genes in this microarray were not significantly affected by RNF20 and RNF40 knockdown and consequent reduction of H2Bub1. Yet, two subgroups of transcripts diverged significantly from the bulk transcriptome. One subgroup was down-regulated upon RNF20 and RNF40 depletion (Fig. 10A, red), implying that H2Bub1 may be preferentially required for their efficient transcription. Interestingly, many transcripts were up-regulated in RNF20- and RNF40-depleted cells (Fig. 10A, blue), suggesting that their transcription is typically suppressed, directly or indirectly, by RNF20 or RNF40. 
A

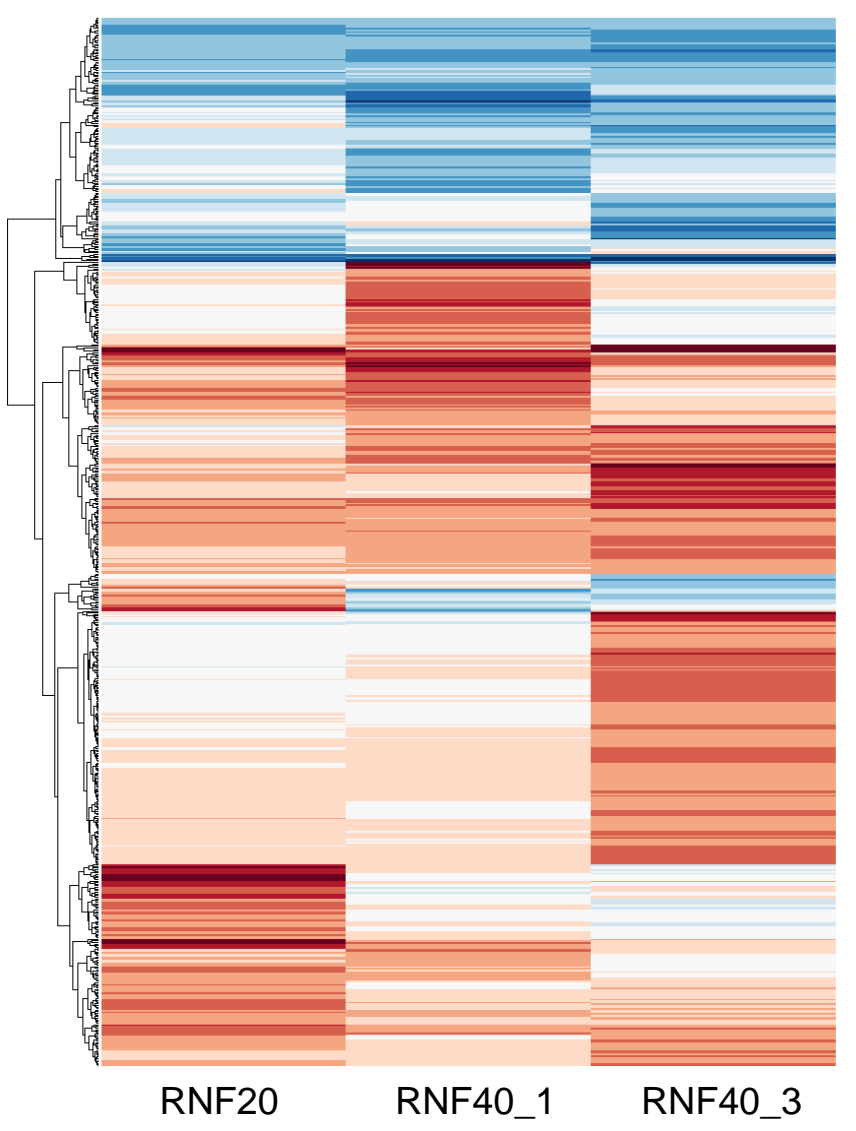

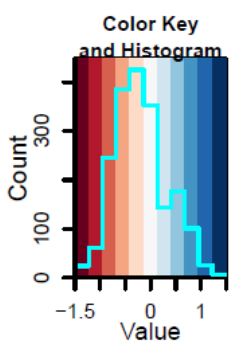

B

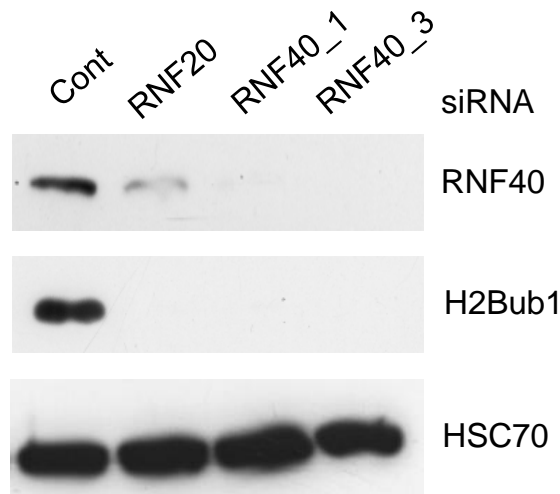

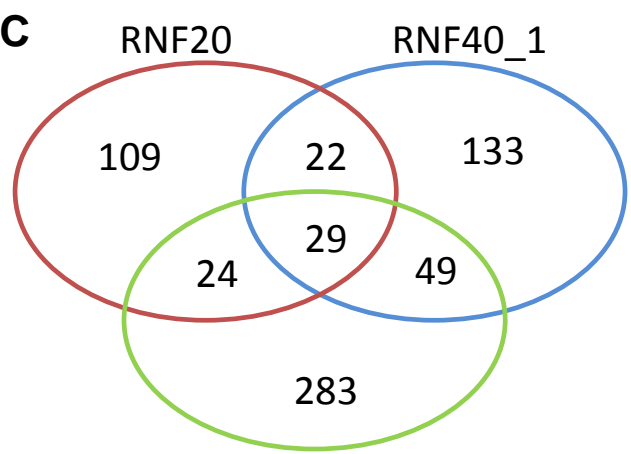

RNF40_3

Fig. 10: mRNA expression profiling of RNF20- and RNF40-regulated genes. (A) The heatmap shows fold-changes in experiments control siRNA vs. RNF20 siRNA, control siRNA vs. RNF40_1 siRNA and control siRNA vs. RNF40_3 siRNA (columns) for genes which are significantly $(q<0.05$; $q$ = $p$-values adjusted to FDR) regulated by RNF20 oder RNF40 knockdown (FC <-log2 (1.5) or FC > log2 (1.5)) (rows). For siRNA-mediated knockdown, MCF10A cells were transfected with $30 \mathrm{pmol}$ control, RNF20, RNF40_1 or RNF40_3 siRNA. Total mRNA was harvested $48 \mathrm{~h}$ after transfection and single-stranded cDNA fragments were generated, biotinylated and hybridized to Affymetrix DNA probe arrays. The arrays were scanned and analyzed as described. The color key is depicted on the top left side ranging from red marking down-regulated to blue marking up-regulated genes; mean values, $n=$ 3. FDR - false discovery rate, FC - fold-changes. (B) Efficient knockdown of RNF20, RNF40_1 and RNF40_3 and the resulting loss of H2Bub1 was confirmed by Western blot analysis using the indicated antibodies. HSC70 served as loading control. (C) Venn-Diagram showing the number of genes significantly regulated ( $\mathrm{q}<0.05, \mathrm{FC}>\log 2(1.5)$ ) between pair-wise comparisons of RNF20, RNF40_1 and RNF40_3 siRNAs. 572 genes were significantly enriched in both comparisons. 
The overlap in genes which were affected by transfection of the RNF20 siRNA or either of the individual RNF40 siRNAs is shown in the Venn-diagram in figure 6C. Only 29 genes were commonly regulated more than $50 \%$ ( -1.5 fold) by all three siRNAs indicating that the maintenance of the MCF10A transcriptional profile occurs in an RNF20 and RNF40 independent manner. The correlation between the microarray data from RNF20 and RNF40 depleted cells and the control cells suggest that $\mathrm{H} 2 \mathrm{~B}$ monoubiquitination is not rate limiting for global transcription in MCF10A cells. These results rule out the hypothesis of general transcription failure upon RNF20 and RNF40 knockdown suggesting its influence in different mechanisms. 14 of the commonly regulated genes were up-regulated while the expression of 15 genes was decreased upon RNF20 and RNF40 depletion.

Together, these results suggest that RNF20 and RNF40 siRNA-mediated reduction of $\mathrm{H} 2 \mathrm{~B}$ monoubiquitination correlates with strong up- or down-regulation of a specific subset of genes.

\subsubsection{RNF20 and RNF40 target gene expression}

The results of the microarray experiment on target gene expression were verified in quantitative RT-PCR (qRT-PCR) studies for three RNF20/RNF40-dependent genes $(A B C C 2, F S T, I L 1 B)$ as well as for a gene whose expression was not affected by RNF20 and RNF40 depletion (gene RPLPO) and was therefore used as a control for normalization. The very efficient reduction of RNF20 and RNF40 transcripts as well as some strongly decreased transcripts of target genes in RNF20 and RNF40 siRNAtransfected cells observed by microarray analysis could be confirmed in qRT-PCR experiments (Fig. 11A and B).

$A B C C 2$ is a member of the superfamily of ATP-binding cassette transporters and is involved in multi-drug resistance. Follistatin (FST) is an autocrine glycoprotein (Tortoriello et al., 2001). Its primary function is the binding of members of the TGF- $\beta$ superfamily, with a particular focus on activin, a paracrine hormone. Secreted TGF$\beta 1$ induces epithelial-to-mesenchymal transition (EMT). The FST gene is a TGF- $\beta$ antagonist found to be down-regulated in a EMT core signature (Taube et al., 2010). Furthermore, the activin A receptor ACVR1C for the TGFB family of signaling molecules is up-regulated in our microarray experiment. Interleukin-1 beta (IL-1 $\beta)$ 
forms a cytokine protein that is an important mediator of the inflammatory response and is involved in a variety of cellular activities, including cell proliferation, differentiation, and apoptosis. $I L-1 \beta$ is forming a complex at the cell membrane with an interleukin 1 receptor and an Interleukin-1 receptor accessory protein (IL1RAP). Expression of the IL1RAP gene was also decreased in the microarray analysis and could be validated by qRT-PCR (data not shown).

A
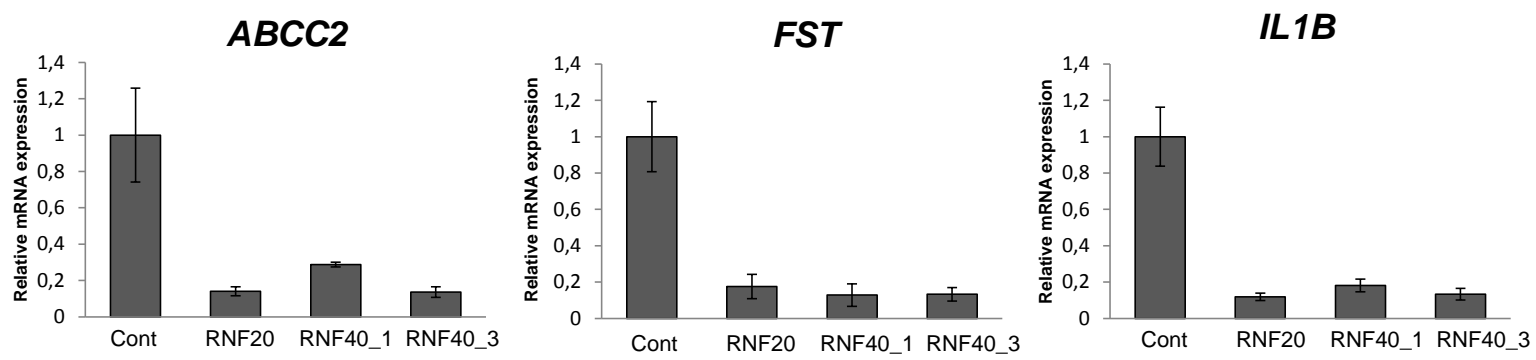

B

RPLPO

RNF40

RNF20
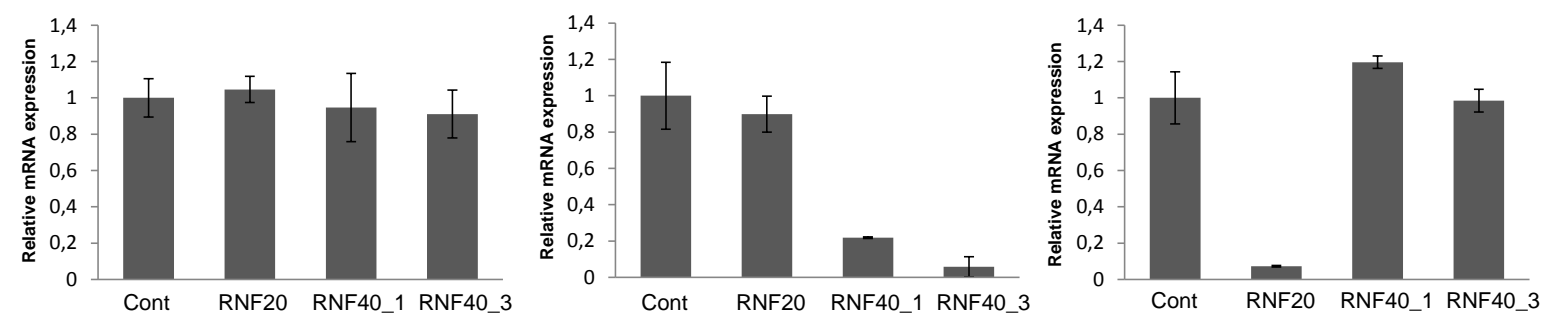

Fig. 11: qRT-PCR validation of selected genes from the microarray experiment. (A) MCF10A cells were transfected with siRNA to RNF20, RNF40 or control siRNA for $48 \mathrm{~h}$. Total mRNA was harvested, reverse-transcribed with random primers and cDNA was subsequently analyzed via q-RTPCR. The expression levels of $A B C C 2, F S T$ and IL1B genes were normalized to RPLPO (control, shown in B) and graphed relative to control sample (set to one). The normalized expression levels were represented as "relative mRNA expression"; mean values $\pm S D, n=3$. (B) Efficient knockdown of RNF20 and RNF40 was confirmed on mRNA level; mean values \pm SD, $n=3$.

\subsubsection{H2Bub1 decreases during tumor progression}

Although a previous study indicated a potential tumor suppressor role for RNF20 (Shema et al., 2008), at the time this project began no study had investigated the presence of H2Bub1 in normal and cancerous tissues. In order to examine the validity of H2Bub1 and its ubiquitin ligase RNF40 as potential tumor suppressors, it was necessary to investigate their expression levels in primary tumor samples. Therefore, we used a new H2Bub1-specific monoclonal antibody using a previously 
published branched-peptide antigen strategy (Minsky et al., 2008) and a commercial available antibody for RNF40 and carried out immunohistochemical analysis of tissue microarrays containing normal tissue as well as malignant cancer samples. As a representative example normal colon tissue versus colon cancer tissue is shown (Fig. 12). Compared to normal colon tissue, nuclear staining of H2Bub1 is decreased in colon cancer tissue (Fig. 12, top panels). Stromal cells are connective tissue cells of any organ and cancer cells often require nearby stromal cells for their own proliferation. As expected, stromal cells visible in this tissue microarray are H2Bub1 positive confirming that the decreased H2Bub1 staining during colon cancer progression is due to decreased monoubiquitination of $\mathrm{H} 2 \mathrm{~B}$ and not due to a lack of sample antigenicity.
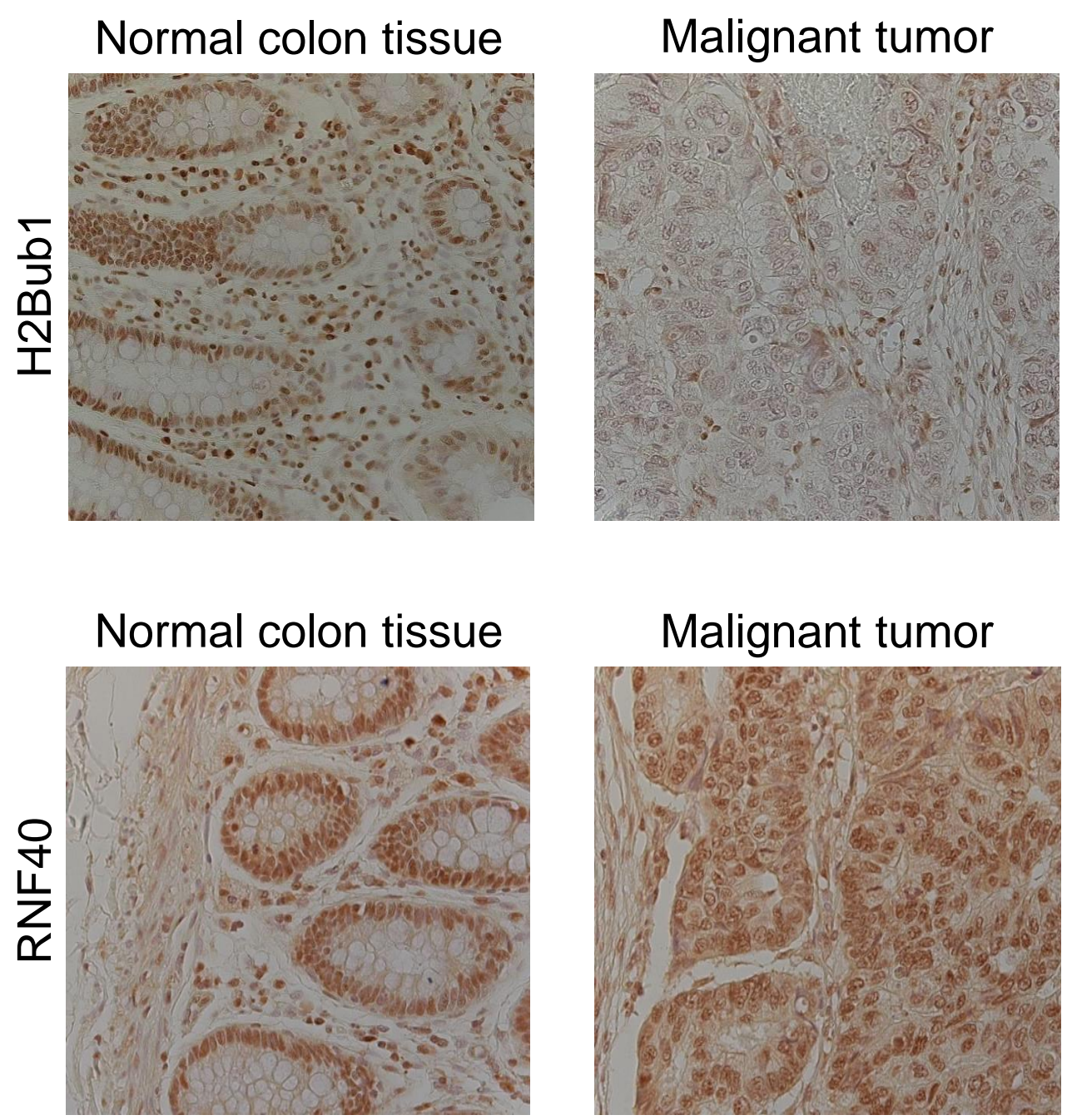

Fig. 12: H2Bub1 and RNF40 expression in human normal colon tissue and tumors. Representative immunohistochemical image displaying H2Bub1 and RNF40 protein expression. 
In addition, the same tissue microarray samples were used for immunohistochemical staining for RNF40. No significant changes in RNF40 levels could be observed between normal and cancer colon tissue (Fig. 12, bottom panels). Since downregulation of RNF20 leads to decreased RNF40 levels we emanate that H2Bub1 regulation in the tissue microarray samples is independent of both RNF20 and RNF40 expression. Thus, it seems that different regulator mechanisms exist since we observed a strong decrease in H2Bub1 nuclear staining in tumor compared to normal tissue. A possible explanation could be the involvement of upstream mechanisms such as the regulation of Cyclin-dependent kinase 9 (CDK9) or WAC which both interact with the RNF20/RNF40 complex and participates in the regulation of H2Bub1 levels (Pirngruber et al., 2009a; Zhang and Yu, 2011). An active suppression of H2Bub1 accumulation is also possible, since in several tumors ubiquitin specific protein 22 (USP22) is overexpressed (Liu et al., 2010, 2011; Zhang et al., 2011) or the RNF20 promoter is hypermethylated down-regulating RNF20 gene expression in breast cancer (Shema et al., 2008). USP22 is a deubiquitinating enzyme and was identified as one of the 'stem cell signature' genes whose overexpression correlates with poor cancer prognosis (Glinsky, 2005). Further analyses of USP22 and the interacting subunit ATXN7L3 which are components of the human SAGA deubiquitinating module complex (Kohler et al., 2010) were done in subsequent experiments.

These preliminary results were further investigated by a colleague and the results were recently published in Prenzel et al., (2011).

Taken together, all these data show a potential tumor-suppressor role for H2Bub1.

\subsubsection{Genomic regulation of ATXN7L3 and USP22 in breast cancer}

ONCOMINE, a cancer microarray database and web-based data-mining platform aimed at facilitating discovery from genome-wide expression analyses. Differential expression analyses comparing most major types of cancer with respective normal tissues as well as a variety of cancer subtypes and clinical-based and pathologybased analyses are available for exploration (Rhodes et al., 2004). ONCOMINE data for USP22 and ATXN7L3 expression in normal breast tissue compared to breast carcinoma tissue showed an increase in the expression level of both USP22 and 
ATXN7L3 in the carcinoma tissue (Fig.13). These results strengthen the hypothesis that efficient deubiquitinases constantly work to remove H2Bub1. The described results are an important basis for further studies on the regulatory mechanism of $\mathrm{H} 2 \mathrm{~B}$ monoubiquitination.

A

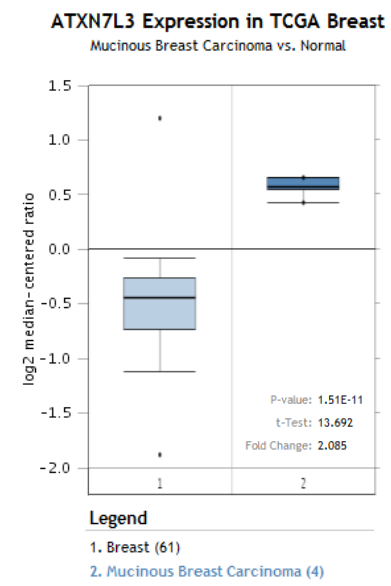

B

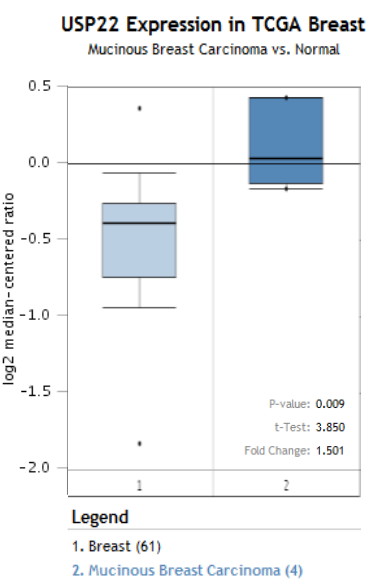

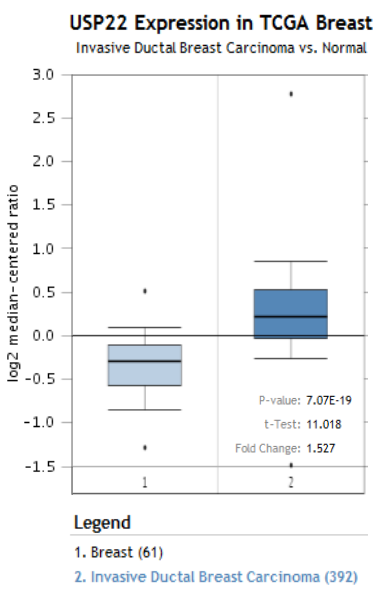

Fig. 13: Genomic regulation of ATXN7L3 and USP22 in breast cancer. Examination of the Oncomine database reveals a significant increase in ATXN7L3 and USP22 levels in different types of breast carcinoma. (Individual P-values and N indicated for each data set.)

\subsection{H2Bub1 levels decrease following various stress conditions}

H2B monoubiquitination is highly dynamic and its level is regulated by addition and removal of the ubiquitin residue from the chromatin.

\subsubsection{Different stress factors rapidly reduce H2B monoubiquitination}

In the course of this study immortalized MCF10A cells, a non-transformed epithelial cell line derived from human fibrocystic mammary tissue and H1299, a human nonsmall cell lung carcinoma cell line were treated with a variety of agents to test their effects on H2Bub1 levels. Earlier reports showed a severe reduction of histone H2Bub1 by a number of different factors. Actinomycin D inhibits transcription via binding DNA at the transcription initiation complex and prevents the elongation of RNA (Sobell, 1985). Several studies demonstrate that Actinomycin D is able to reduce H2Bub1 levels already at low concentrations rapidly confirming the importance of active transcription on maintaining global H2Bub1 levels (Davie and 
Murphy, 1990; Minsky et al., 2008; Pirngruber et al., 2009a). The study from Davie and Murphy (1990) showed a loss of monoubiquitinated H2B upon heat-shock treatment as well. Further, DNA damage was also suggested to reduce H2Bub1 levels (Lis and Romesberg, 2006) and Minsky et al. (2008) showed a significant decrease in H2Bub1 upon Doxorubicin treatment. In agreement with these reports we observed a drop in this histone mark by Actinomycin D, Doxorubicin and heat-shock treatment as well (Fig. 14). Consistent with these studies, we could show that the important anticancer drug Cisplatin (Fig. 14A), which is able to form adducts with DNA (Kartalou and Essigmann, 2001) reduces global H2Bub1 levels as well. In addition, the present study demonstrated that osmotic stress in terms of Sorbitol treatment nearly abolish H2Bub1 levels dose dependently as shown in figure 14A. To establish which dose is necessary to reduce the level of H2Bub1 in MCF10A cells, concentration courses were performed. Based on these data, all the following stressinducing experiments were performed using $20 \mathrm{nM}$ Actinomycin $\mathrm{D}$ and $50 \mu \mathrm{M}$ Cisplatin.

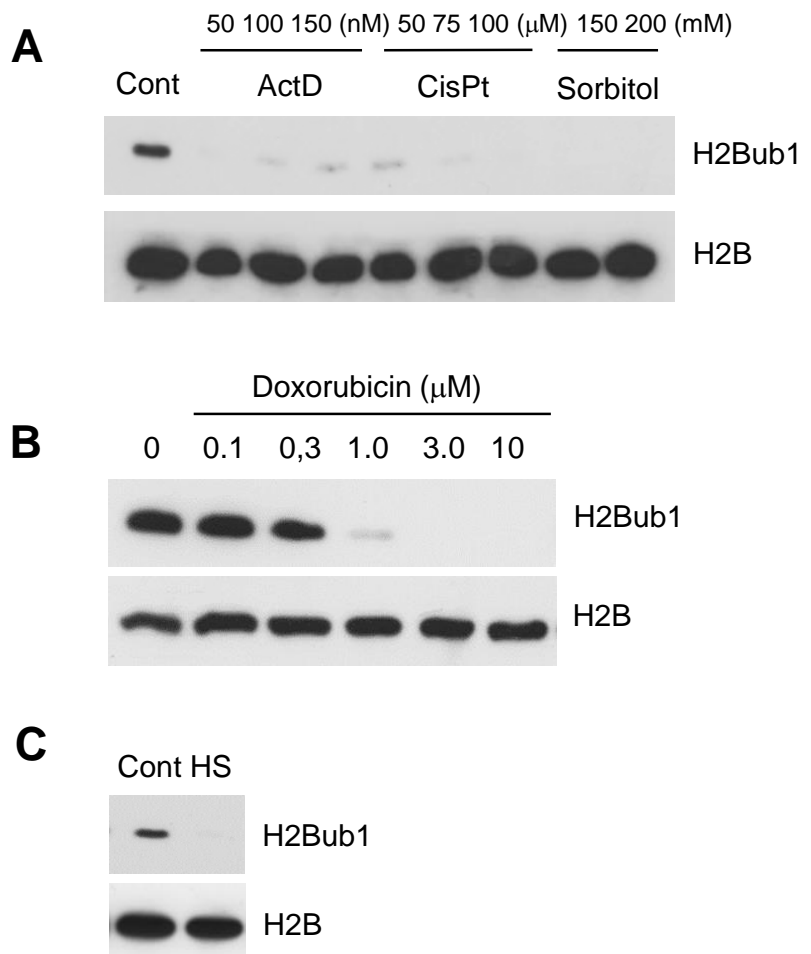

Fig. 14: Various factors down-regulate H2B ubiquitination. (A) MCF10A cells were treated for 12 $\mathrm{h}$ with increasing concentrations of Actinomycin D, Cisplatin and Sorbitol. Whole protein extract were harvested and analyzed via Western blot using specific H2Bub1 antibody. H2B is shown as a loading control. (B) HCT116 cells were treated with the indicated concentrations of Doxorubicin for $6 \mathrm{~h}$. Protein extracts were subjected to Western blot analysis. (C) MCF10A cells were heat shocked (HS) by incubation at $42^{\circ} \mathrm{C}$ for $1 \mathrm{~h}$. Protein extracts were analyzed by Western blot. 


\subsubsection{Treatment with stress-inducing factors alters RNF20- and RNF40- regulated gene transcription}

In order to test whether the expression of RNF20- and RNF40- regulated genes are also influenced by cellular stress which decrease H2Bub1 levels, MCF10A cells were treated with Actinomycin D and Cisplatin. To verify the efficient loss of H2Bub1 upon treatment protein extracts were analyzed via Western blot using a specific antibody against H2Bub1 (Fig. 15A). Examination of the mRNA levels of the ABCC2, FST and IL1B genes by qRT-PCR showed a strong decrease in gene transcripts upon treatment, while RPLPO as a control remained largely unaffected or slightly increased (Fig. 15B).
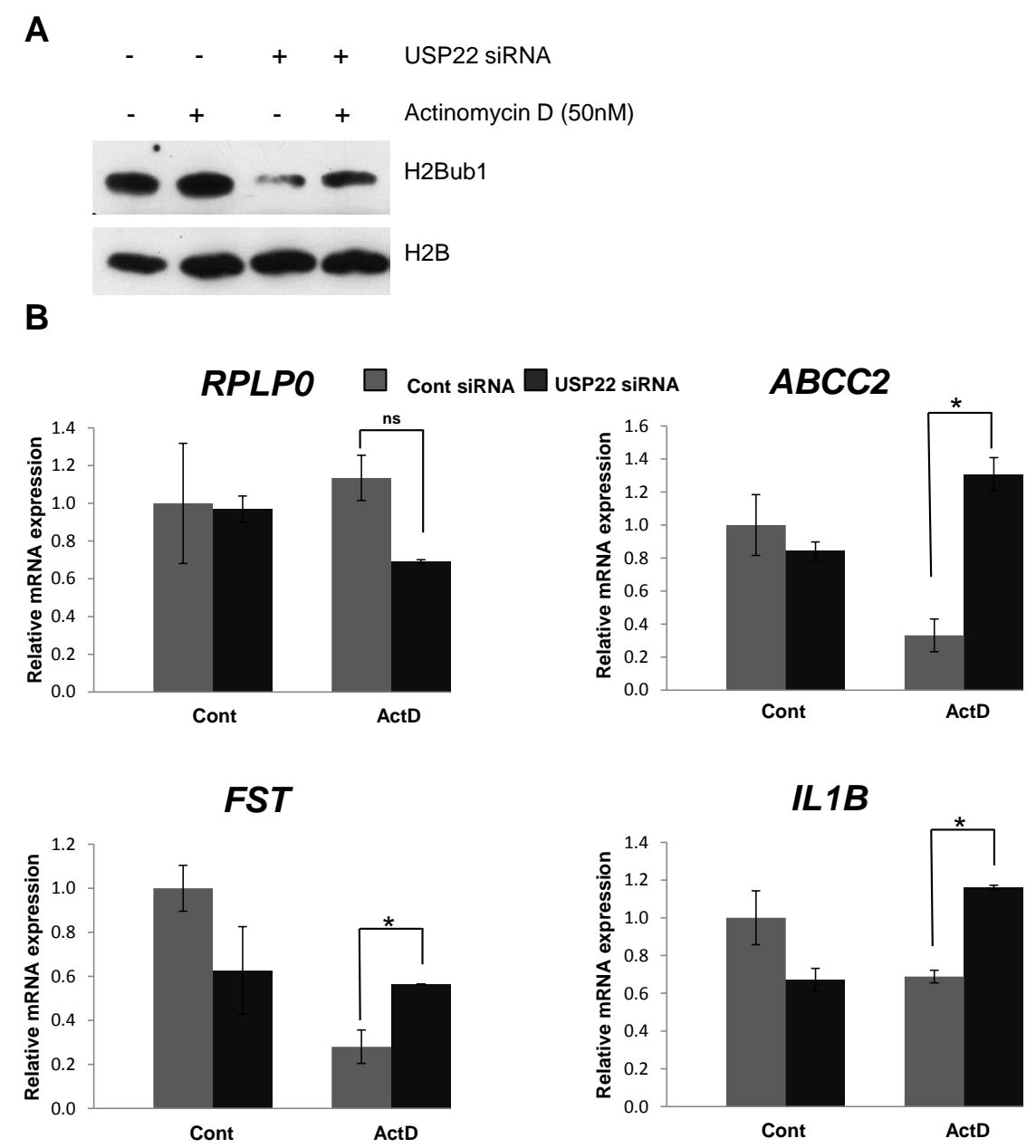

Fig. 15: Different stress factors decrease RNF20 and RNF40 target gene expression. (A) MCF10A cells were treated with $20 \mathrm{nM}$ Actinomycin D for $3 \mathrm{~h}$ or $50 \mathrm{mM}$ Cisplatin for $12 \mathrm{~h}$. mRNA samples were analyzed via qRT-PCR and expression levels were represented as described in figure 11; mean values $\pm S D, n=3$. (B) Whole protein extracts were analyzed via Western blot using antibodies against H2Bub1 and $\mathrm{H} 2 \mathrm{~B}$ as a loading control. 


\subsubsection{Actinomycin D affects rRNA transcription}

Most drugs require specific transport mechanisms and other stress inducing factors need subsequent steps to get inside the cell and cause transcriptional changes. In order to analyze the dynamics on global H2Bub1 level upon ribosomal stress we treated cells with Actinomycin D. From the results of a time course treatment it is clear that a significant loss of H2Bub1 already happens within 30 min after treatment (Fig. 16B) indicating that H2B ubiquitination and deubiquitination are very fast and highly dynamic processes.

At low concentrations Actinomycin $\mathrm{D}$ is a potent RNAPI inhibitor and leads to ribosomal stress via rapidly disrupting the function of nucleoli by blocking ribosomal RNA transcription (Perry \& Kelley, 1968). Concentration course studies showed that the decrease of H2Bub1 and activation of the stress-responsive kinase JNK happen in the same range of concentrations (Fig. 16C). The JNK pathway is introduced in the subsequent chapter 4.3.1. Since H1299 cells used in the experiment were p53negative the observed effects are independent of the p53 pathway.

To determine if loss of global levels of H2Bub1 correlates with the inhibition of RNAPI we checked for transcribed rRNA genes. 18S, 5.8S, and 28S rRNAs are transcribed as a single large precursor molecule (Fig. 16A). Processing steps of this pre-rRNA are investigated to check how fast Actinomycin $D$ influences the rRNA transcription rate. During the processing transcribed spacer RNA gets removed and is rapidly degraded. Evaluation of the internal transcribed spacer (ITS) ITS 2 to 28S, the 5'ETS (external transcribed spacer) to $18 \mathrm{~S}$ and $5.8 \mathrm{~S}$ to ITS2 RNA expression level using qRT-PCR revealed, that Actinomycin $D$ treatment resulted in a decreased rRNA transcription even after $30 \mathrm{~min}$ (Fig. 16D). To normalize the data primers for total $28 \mathrm{~S}$ rRNA were used. Thus, inhibition of RNAPI activity coincides with decreased H2Bub1 levels and activation of the stress-responsive kinase JNK (Fig. 16B). 
A

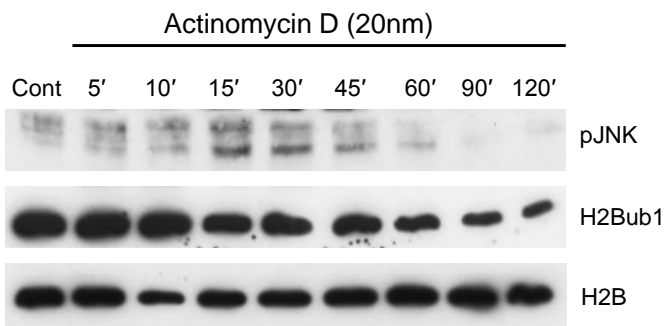

B

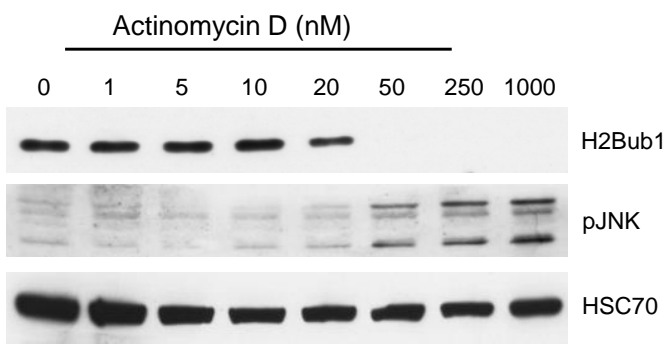

D

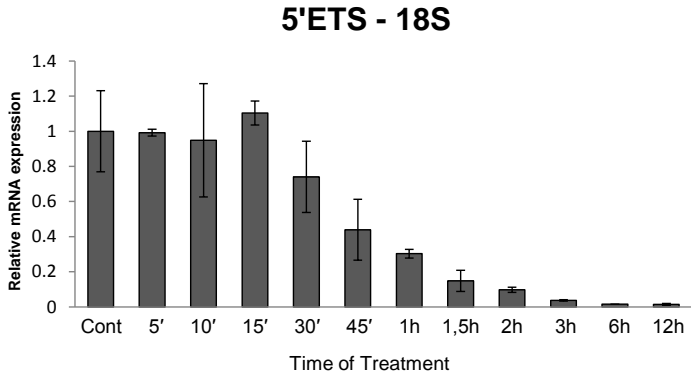

$5.8 S$ - ITS 2

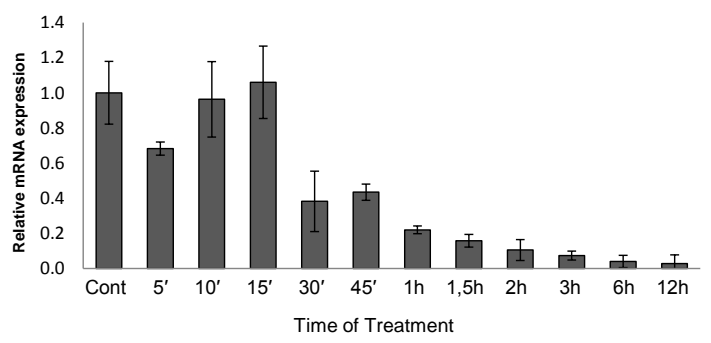

C
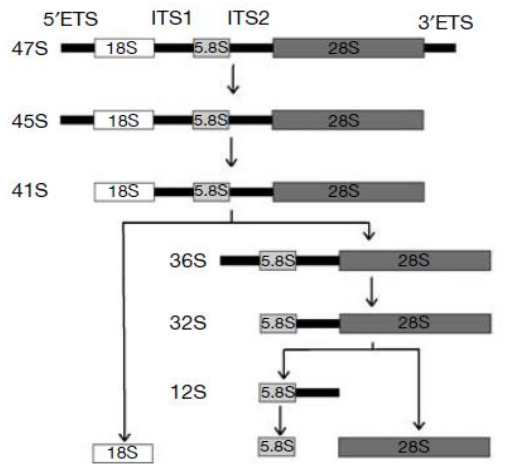

ITS 2 - $28 S$

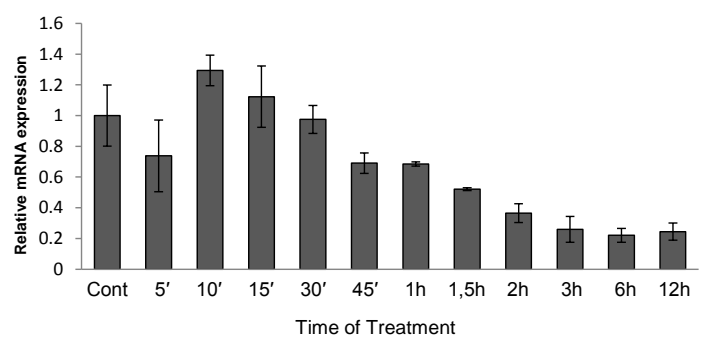

Fig. 16: H2Bub1 reduction happens very rapidly and affects rRNA transcription. (A) Scheme of the assembly of rRNA into the ribosomal subunits. In eukaryotes, each cytoplasmic ribosome comprises four different molecules of RNA: two large rRNAs designated 18S and $28 \mathrm{~S}$ and two small rRNAs termed $5 S$ and 5.8S. While all four rRNAs of cytoplasmic ribosomes are encoded in nuclear genes, the 18S, 5.8S, and 28S rRNAs are transcribed as a single large precursor molecule. Posttranscriptional processing of this pre-rRNA in the nucleolus subsequently yields separate mature rRNAs that assemble with numerous ribosomal proteins to form the ribosomal subunits (modified from Gillespie et al. (2006)). (B) MCF10A cells were treated with $20 \mathrm{nM}$ Actinomycin D and harvested for Western blot after indicated times. (C) MCF10A cells were treated with indicated concentrations of Actinomycin $D$ for $12 \mathrm{~h}$. Proteins were analyzed by Western blot using the indicated antibodies. (D) MCF10A cells were treated with $20 \mathrm{nM}$ Actinomycin D for the indicated times. Total mRNA was harvested, reverse-transcribed with random primers and analyzed with qRT-PCR. The expression levels were normalized to $28 \mathrm{~S}$ ribosomal RNA as an internal reference gene. The normalized expression levels are graphed relative to control sample which is set to one. The expression levels are represented as "relative mRNA expression". ETS - external transcribed spacer; ITS - internal transcribed spacer. Mean values $\pm S D, n=3$. 


\subsubsection{Actinomycin D concentration specifically affects RNF20 and RNF40 target gene transcription}

Actinomycin D is an antibiotic known to inhibit RNAPI at low doses and RNAPII at high doses. In order to check whether the utilized level of Actinomycin $D$ already inhibits the general RNAPII transcription we evaluated heterogeneous nuclear RNA (hnRNA) using primers which span exon-intron boundaries by qRT-PCR. Eukaryotic pre-mRNA includes two different types of segments, exons and introns and it exist only briefly before it is fully processed into mRNA. Thus hnRNA levels closely reflect the transcriptional activity of the investigated gene. Consistent with the observed decrease in H2Bub1 a highly significant reduction in mRNA expression of the RNF20 and RNF40 target genes $A B C C 2, F S T$ and $I L 1 B$ upon treatment of Actinomycin $D$ for either $3 \mathrm{~h}$ or $6 \mathrm{~h}$ was detected. The expression of RPLPO was used as a negative control and remained largely unchanged (Fig. 17).

hnRPLPO

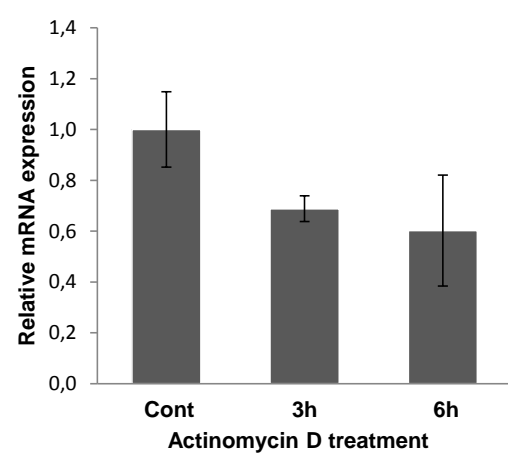

hnFST

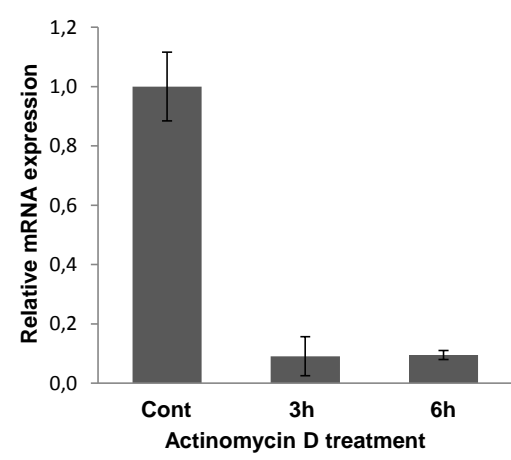

hnABCC2

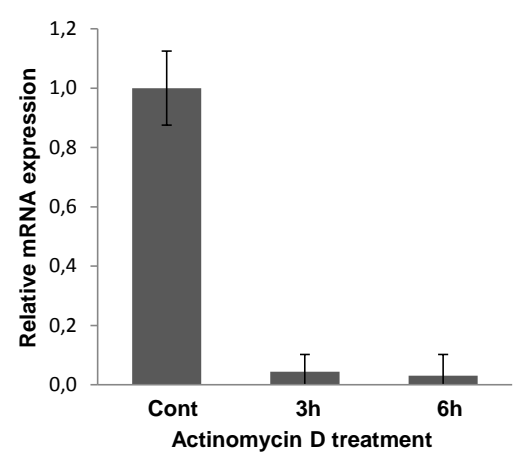

hnIL1B

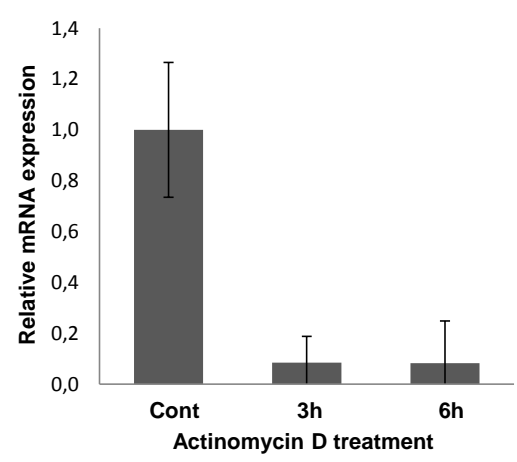

Fig. 17: RNF20 and RNF40 target gene expression but not general RNAPII transcription is affected upon Actinomycin D treatment. MCF10A cells were treated with $20 \mathrm{nM}$ Actinomycin $D$ for $3 \mathrm{~h}$ and $6 \mathrm{~h}$. RNA was isolated and cDNA was analysed by qRT-PCR using hn primers. Gene expression was normalized to hnRPLPO expression (indicated as "relative mRNA expression"). hn heterogeneous nuclear. Mean values $\pm S D, n=3$. 


\subsubsection{Cisplatin affects H2Bub1 occupancy}

The medical relevance of Cisplatin in anticancer treatment disposes us to investigate the effect of Cisplatin treatment on H2Bub1 occupancy in MCF10A cells by performing chromatin-immunoprecipitation (ChIP) experiments. Two highly expressed genes, RPLPO and GAPDH demonstrated decreased H2Bub1 occupancy on their transcriptional start sites (TSS) and their transcribed regions (TR) (Fig. 18). In general, H2Bub1 is higher at the TR compared to the TSS consistent with previous reports which showed that H2Bub1 in humans is preferentially found in the transcribed regions of highly expressed genes and is therefore suggested to be linked to transcriptional elongation (Fleming et al., 2008; Minsky et al., 2008). After verifying the efficient $\mathrm{H} 2 \mathrm{Bub} 1$ reduction the level of trimethylated lysine 36 in histone H3 (H3K36me3), which was used as a control, was examined. No difference in H3K36me3 occupancy in the presence or absence of Cisplatin could be observed. Trimethylation of $\mathrm{H} 3 \mathrm{~K} 36$ is associated with the coding region of genes and correlates with active transcription (Krogan et al., 2003). The connection to transcriptional elongation and the independence on the H2Bub1 driven transhistone pathway (Briggs et al., 2002) makes H3K36me3 to a useful control to show that global histone modifications are unaffected. 


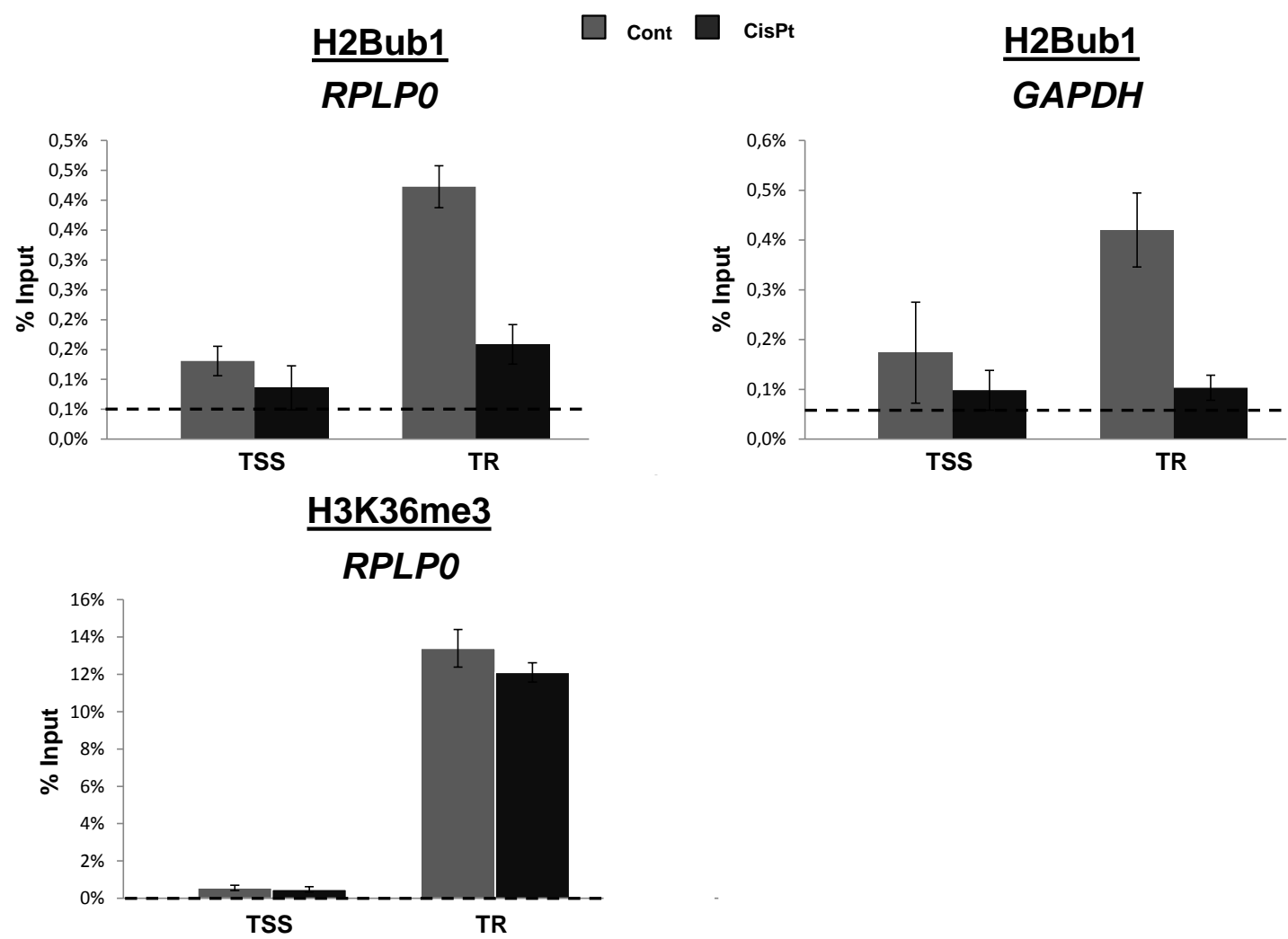

Fig. 18: Chromatin immunoprecipitation (ChIP) analysis revealed a decrease in H2Bub1 occupancy upon Cisplatin treatment. Chromatin extracts were analyzed by ChIP for H2Bub1 and H3K36me3 using a specific antibody. Non-specific IgG was used to distinguish between specific and background binding (depicted as dotted line). ChIP samples were normalized to input samples and expressed as "percent input". Mean values $\pm S D, n=3$.

In summary, RNF20 and RNF40 dependent genes and the H2Bub1 level is reduced upon Cisplatin treatment, but global histone modifications like H3K36me3 seems to be unaffected.

\subsubsection{RNAPI inhibitor decreases H2Bub1 level similar to Actinomycin D}

To verify if the loss of H2Bub1 upon Actinomycin D treatment is because of RNAPI inhibition and not due to possible side effects, a specific RNAPI inhibitor which selectively inhibits rRNA synthesis in the nucleolus, but does not inhibit mRNA synthesis was used. Thus, selective inhibition of RNAPI by CX-5461 may offer a general therapeutic strategy to block cancer cell proliferation, as potent antiproliferative activity in cancer cells and xenografts is described (Drygin et al., 2011). We found that already $15 \mathrm{~min}$ after nucleolar stress induction via the RNAPI inhibitor CX-5461 the H2Bub1 levels was strongly reduced, and even more efficiently after 45 
min compared to the non-treated control cells (Fig. 19). Taken together, these results demonstrate that nucleolar stress-inducing factors inhibiting RNAPI decreases the H2Bub1 level globally, while the general RNAPII transcription remains unaffected.

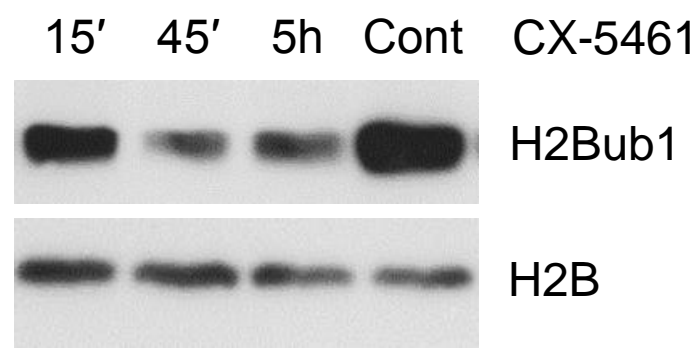

Fig. 19: The specific RNAPI inhibitor CX-5461 decreases H2Bub1 level. MCF10A cells were treated with $20 \mathrm{mM} \mathrm{CX}-5461$. H2Bub1 protein levels were analyzed by Western blotting using specific antibody against H2Bub1. H2B served as loading control.

\subsection{Cellular stress activates several signaling pathways}

Chromatin modifications alter in response to different stresses. Since the concentration of stress inducing factors where cells develop a strong DNA-damage response is much lower than the concentration where H2Bub1 levels were affected, we analyzed several signaling pathways which are known to be activated by cell stress and potentially lie downstream of the DNA-damage response to determine if they are involved in the stress-induced loss of H2Bub1.

\subsubsection{JNK activity regulates the level of H2B ubiquitination}

Activation of c-Jun N-terminal kinases (JNKs) is a hallmark of various stress inducers. Inflammatory signals, for example exposure to UV-B radiation, protein synthesis inhibitors, and a variety of stress stimuli can activate JNK (Song and Lee, 2007; Yoon and Kim, 2004). To determine whether increased JNK activation might be essential for the stress-induced loss of H2Bub1 we used a specific JNK inhibitor SP600125. Indeed, pretreatment with the JNK inhibitor partially blocked the ability of Actinomycin D to decrease global H2Bub1 levels (Fig. 20). Decreased protein levels of phosphorylated JNK upon JNK inhibitor treatment compared to the untreated control MCF10A cells confirmed the efficiency of the JNK inhibitor. 


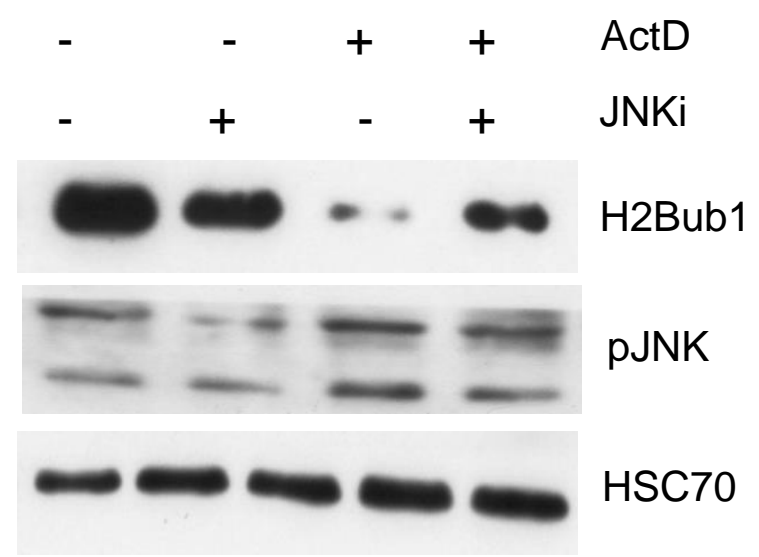

Fig. 20: JNK inhibition interferes with Actinomycin D-inducible H2Bub1 deubiquitination. MCF10A cells were pretreated with $20 \mu \mathrm{M}$ of JNK inhibitor SP600125 for $1 \mathrm{~h}$ and then treated with 256 $\mathrm{nM}$ of Actinomycin D for $3 \mathrm{~h}$. Protein extracts were harvested and analyzed by Western blotting. Phospho-JNK shows two bands since the antibody detects endogenous levels of JNK dually phosphorylated at threonine 183 and tyrosine 185.

\subsubsection{Regulation of H2B ubiquitination upon AMP signaling}

Metabolic reprogramming is associated with tumorigenesis and tumor metabolism (Shackelford and Shaw, 2009). The AMP-activated protein kinase (AMPK) is a metabolic sensor that helps maintain cellular energy homeostasis. AMPK activity has been recently linked to stress resistance and survival in tumor cells (Jeon et al., 2012; Liu et al., 2012).

Since AMPK was reported to activate transcription through direct association with chromatin and phosphorylation of histone $\mathrm{H} 2 \mathrm{~B}$ at serine 36 , we asked whether the AMPK signaling pathway is also involved in the stress-induced loss of H2Bub1 (Bungard et al., 2010). In order to address this question we tested whether known activators for AMPK can influence global H2Bub1 levels. For the stimulation of the AMPK activity, two different reagents were used: Metformin, a glucose-lowering drug which is in clinical use for the treatment of type 2 diabetes; and AICAR (Acadesine), an analog of adenosine (Webster et al., 2010). Changes in AICAR levels in cancer cells alter cellular energy level and increases AMPK-dependent glucose uptake and lactate production (Keller et al., 2012). 


\subsubsection{AICAR treatment leads to loss of H2Bub1 levels}

Time course experiments with AICAR in $\mathrm{H} 1299$ cells revealed a dramatic reduction of H2Bub1 levels as early as $30 \mathrm{~min}$ and $1 \mathrm{~h}$ after AICAR treatment. The level of H2Bub1 recovered during the subsequent observation period of up to $24 \mathrm{~h}$. These data indicate again that $\mathrm{H} 2 \mathrm{~B}$ ubiquitination and deubiquitination are very fast and highly dynamic processes (Fig. 21A). Surprisingly, Metformin treatment did not appreciably affect H2Bub1 levels (Fig. 21B). This leads to the hypothesis that AICARinduced loss of H2Bub1 levels is not dependent on AMPK activity per se.

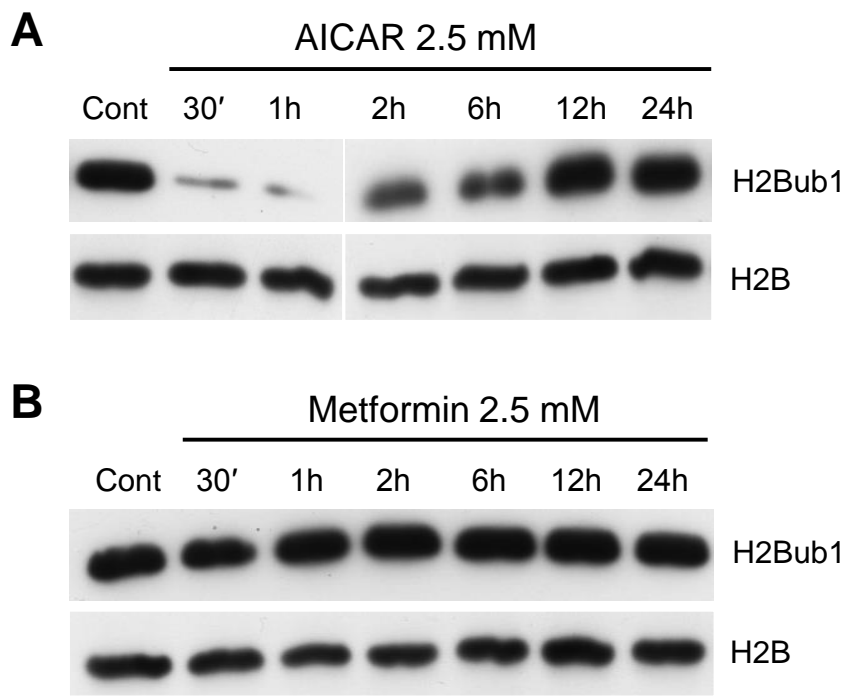

Fig. 21: AICAR treatment but not Metformin leads to reduced H2Bub1 levels. H1299 cells were treated either with $2.5 \mathrm{mM}$ AICAR (A) or $2.5 \mathrm{mM}$ Metformin (B) for the indicated times. H2Bub1 protein levels were analyzed by Western blotting using specific antibody against the H2Bub1. H2B served as loading control.

\subsubsection{The effect of upstream kinases on H2Bub1 level}

To further investigate the mechanisms that regulate H2Bub1 levels we determined whether the effects of AICAR are mediated by AMPK. Therefore we performed siRNA-mediated depletion of AMPK $\alpha 1$ and/or AMPK $\alpha 2$ and analyzed the level of H2Bub1 upon AICAR treatment in whole protein extracts by Western blot. Figure 22A shows that AICAR treatment leads to reduced global H2Bub1 protein levels but siRNAs targeted to AMPK $\alpha 1$ and $A M P K \alpha 2$ or a mixture of both do not increase the down-regulated H2Bub1 levels upon treatment compared to control siRNA transfected MCF10A cells. The efficient knockdown of AMPK $\alpha 1$ was confirmed using 
AMPKa1 antibody in Western blot analysis. Unfortunately, it was not possible to check the activation of AMPK by using phospho-AMPK antibody, as no clear signal was detectable.

The tumor suppressor LKB1 is an important upstream serine/threonine kinase that phosphorylates and activates the AMPK complex (Hawley et al., 2003). Although AMPK activation is lost when LKB1 expression is silenced, the basal activity of AMPK remains intact (Hawley et al., 2003). Cells lacking LKB1 display defective energydependent AMPK activation (Shaw et al., 2004). Similar to the results of the AMPKknockdown experiment, H2Bub1 levels are decreased upon AICAR treatment but no remarkable increase in H2Bub1 was detectable upon siRNA-mediated knockdown of LKB1 compared to control-transfected cells in the presence or absence of AICAR (Fig. 22B).

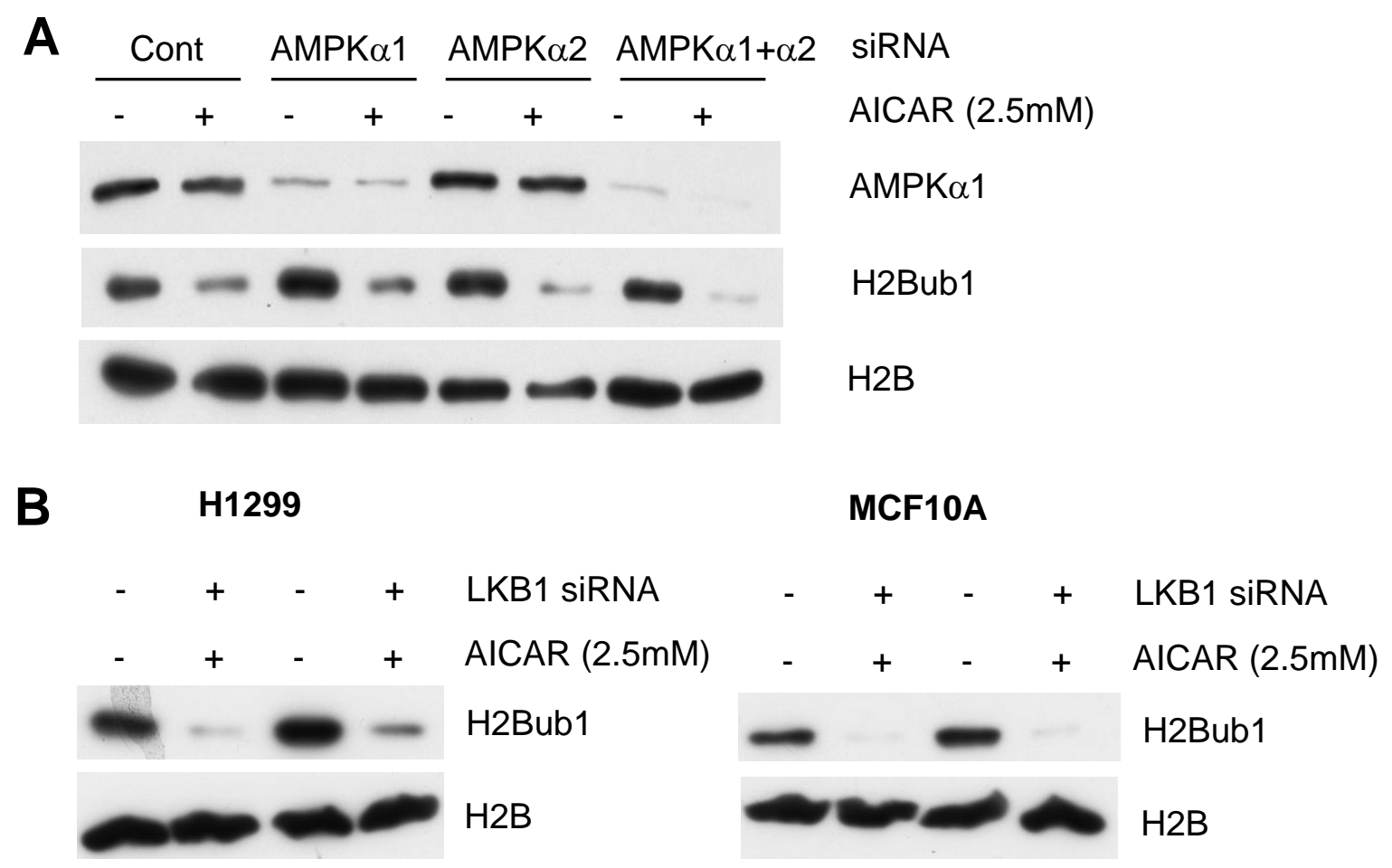

Fig. 22: LKB1 and AMPK depletion don't affect the H2Bub1 level. (A) MCF10A cells were reversetransfected with $30 \mathrm{pmol}$ control, AMPK $\alpha 1$, AMPK $\alpha 2$ or combined AMPK $\alpha 1+\alpha 2$ siRNA for $72 \mathrm{~h}$ followed by $2.5 \mathrm{mM}$ AICAR treatment for $30 \mathrm{~min}$. Protein levels were analyzed by Western blot using specific antibody against H2Bub1, AMPK $\alpha 1$ and H2B as a loading control. (B) MCF10 and H1299 cells were transfected with siRNA to LKB1 and treated with $2.5 \mathrm{mM}$ AICAR for $30 \mathrm{~min}$. H2Bub1 protein levels were analyzed by Western blotting using specific antibody against H2Bub1. H2B served as loading control. 
Summing up, these data indicate that the loss of H2Bub1 levels upon treatment of different stress-inducing factors might be less due to changes in AMPK levels and more due to other AMP signaling events.

\subsubsection{Effect of AICAR on RNF20 and RNF40 target gene transcription}

Therefore we addressed the question how AICAR-induced H2Bub1 levels are regulated. Based on the AICAR dosage course shown in figure $21 \mathrm{~A}$ all following experiments were performed using AICAR treatment for $30 \mathrm{~min}$ in order to assure efficient reduction in H2Bub1.

First, we examined whether AICAR treatment changes expression of RNF20 and RNF40 dependent genes. Similar as shown above for Actinomycin D and Cisplatin, AICAR treatment also led to a decrease in the mRNA levels of the FST, ABCC2 and IL1B genes, but not the control gene RPLPO (figure 23A).

A
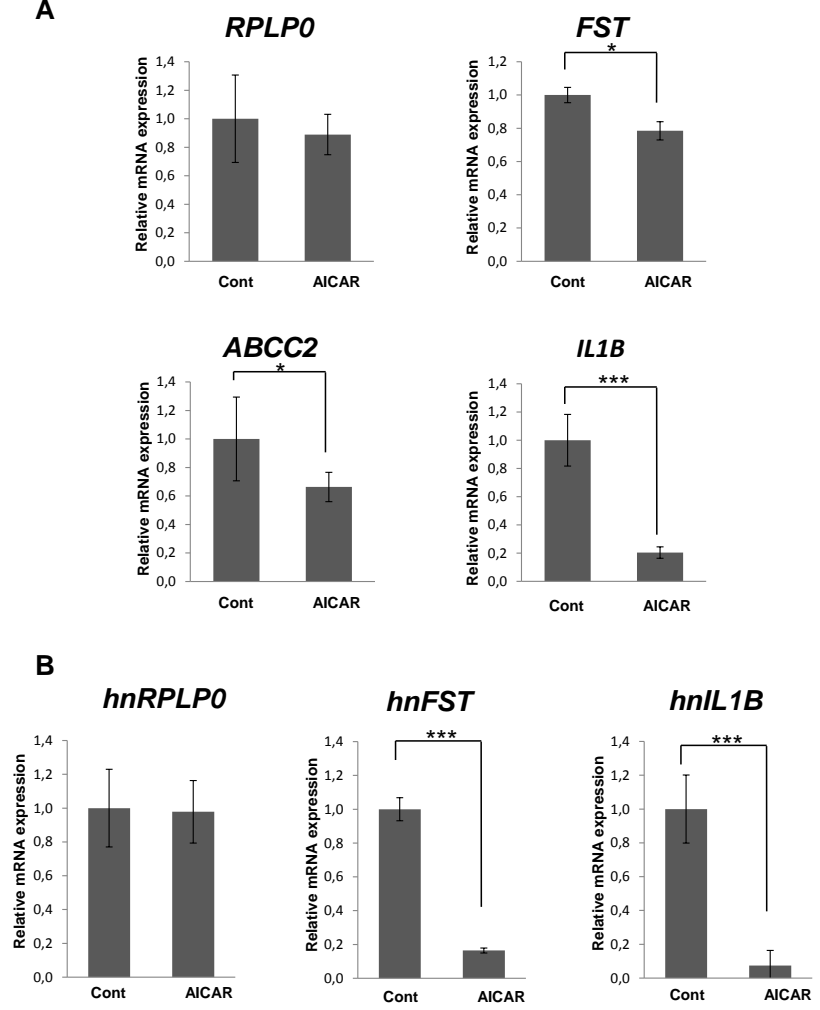

Fig. 23: AICAR treatment leads to reduced RNF20 and RNF40 target gene expression. (A) MCF10A cells were treated with $2.5 \mathrm{mM}$ AICAR for $6 \mathrm{~h}$. mRNA levels were analyzed by qRT-PCR. Gene expression was normalized to RPLPO expression (indicated as "Relative mRNA expression") Mean $\pm S D, n=3$. (B) MCF10A cells were treated with $2.5 \mathrm{mM}$ AICAR for $30 \mathrm{~min}$. mRNA levels were analyzed by qRT-PCR. The expression levels were normalized to hnRPLPO. Statistically significant differences are indicated: ${ }^{*} P \leq 0.05 ;{ }^{* *} P \leq 0.01 ;{ }^{* * *} P \leq 0.001$; mean $\pm \mathrm{SD}, \mathrm{n}=3$. 
In order to test whether the observed effects on mRNA expression levels of RNF20/RNF40 target genes is transcriptional, and if the weak effects shown in figure 23, e.g. for FST become more apparent we looked for hnRNA expression using hn primers, which spans exon-intron boundaries. We observed an even more efficient decrease in ILIB and FST hnRNA levels upon AICAR treatment, whereas the RPLPO control gene remained unaffected (Fig. 23B).

\subsubsection{AICAR affects H2Bub1 occupancy of RNF20/RNF40 target genes}

We next performed ChIP analysis to examine if the effects of AICAR treatment on RNF20 and RNF40 target gene expression correlate with decreased H2Bub1 occupancy levels on these genes. In case of the three representative genes $A B C C 2$, IL1B, FST and RPLPO and GAPDH as control genes, we could observe a clear correlation between H2Bub1 occupancy and expression levels of RNF20 and RNF40 target genes. AICAR treatment resulted in a decreased H2Bub1 occupancy on all five genes either at the transcriptional start site (TSS) or the transcribed region (TR) (Fig. 24). The control genes GAPDH and RPLPO were not affected in gene expression experiments so it could be argued that high H2Bub1 levels are maintained on those genes upon AICAR treatment, despite a drop in overall H2Bub1 shown by ChIP data.

Taken together these results examined that all genes independent of their transcriptional response to RNF20 and RNF40 depletion, exhibited a significant reduction in associated H2Bub1 upon AICAR treatment. 


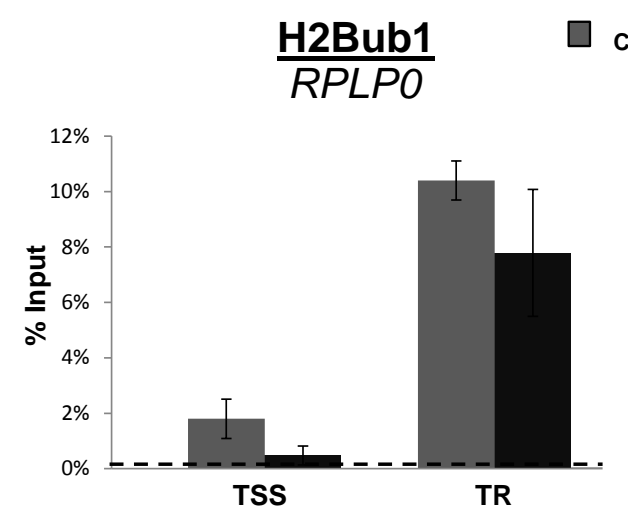

AICAR

H2Bub1

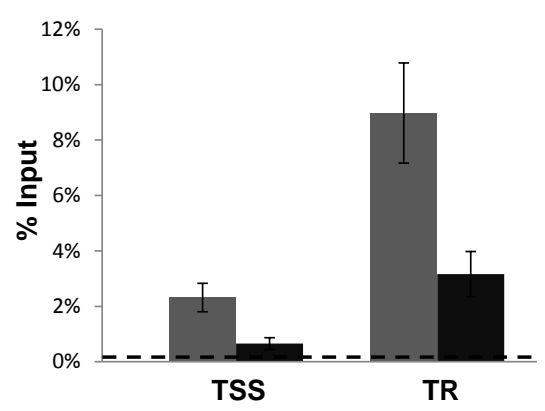

H2Bub1

ABCC2

$\underline{\text { H2Bub1 }}$

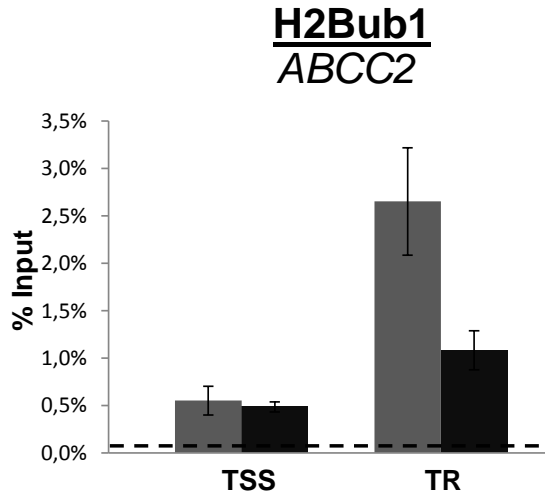

FST

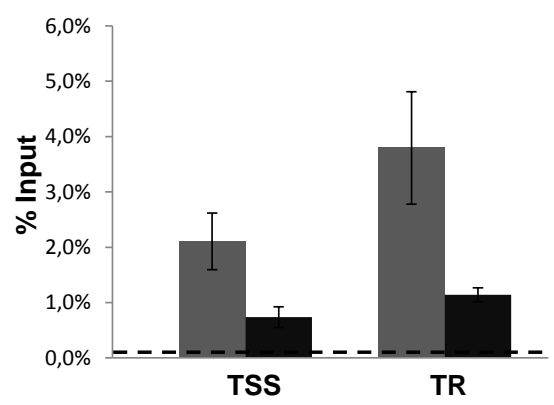

$\underline{\text { H2Bub1 }}$

IL1B

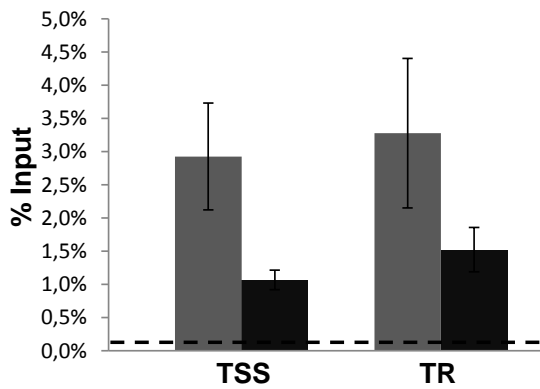

Fig. 24: H2Bub1 levels decrease upon AICAR treatment. MCF10A cells were treated with AICAR for $30 \mathrm{~min}$. Chromatin extracts were analyzed by ChIP for presence of H2Bub1. IgG antibody was used to determine background binding (indicated as dotted line). H2Bub1 occupancy was normalized to input (indicated as "\% of input"). Mean $\pm S D, n=3$.

\subsubsection{Involvement of the glucose metabolism in regulating global H2Bub1 levels}

Stressful conditions deplete ATP stores and increase AMP content in cells. AMPK is activated in an AMP-dependent manner and increased AMPK activity results in the stimulation of glucose uptake in muscle (Musi et al., 2002). To assess possible 
changes of ATP production in stress-induced enzymatic reactions upon glucose starvation and in glucose starvation combined with 2-Deoxy-D-Glucose (2-DG) treatment, an ATP determination kit was used. The bioluminescence assay is based on luciferase's requirement for ATP to produce light. 2-DG is a specific blocker for glycolysis without altering other nutrients or metabolic pathways and its phosphorylation leads to depletion in cellular ATP. In addition, 2-DG is reported to activate AMPK (Wang et al., 2011).

The analysis of the ATP assay revealed that glucose starvation itself already leads to a reduction in the intracellular ATP production (Fig. 25A). 2-Deoxy-glucose treated MCF10A cells grown in glucose-free medium showed a ten-fold down-regulation of cellular ATP levels compared to the control and a five-fold decrease compared to glucose starved MCF10A cells. Next, we examined the effect of 2-DG treatment on H2Bub1 protein level and indeed we could observe reduced H2Bub1 levels compared to control and to glucose starved MCF10A cells (Fig. 25B).

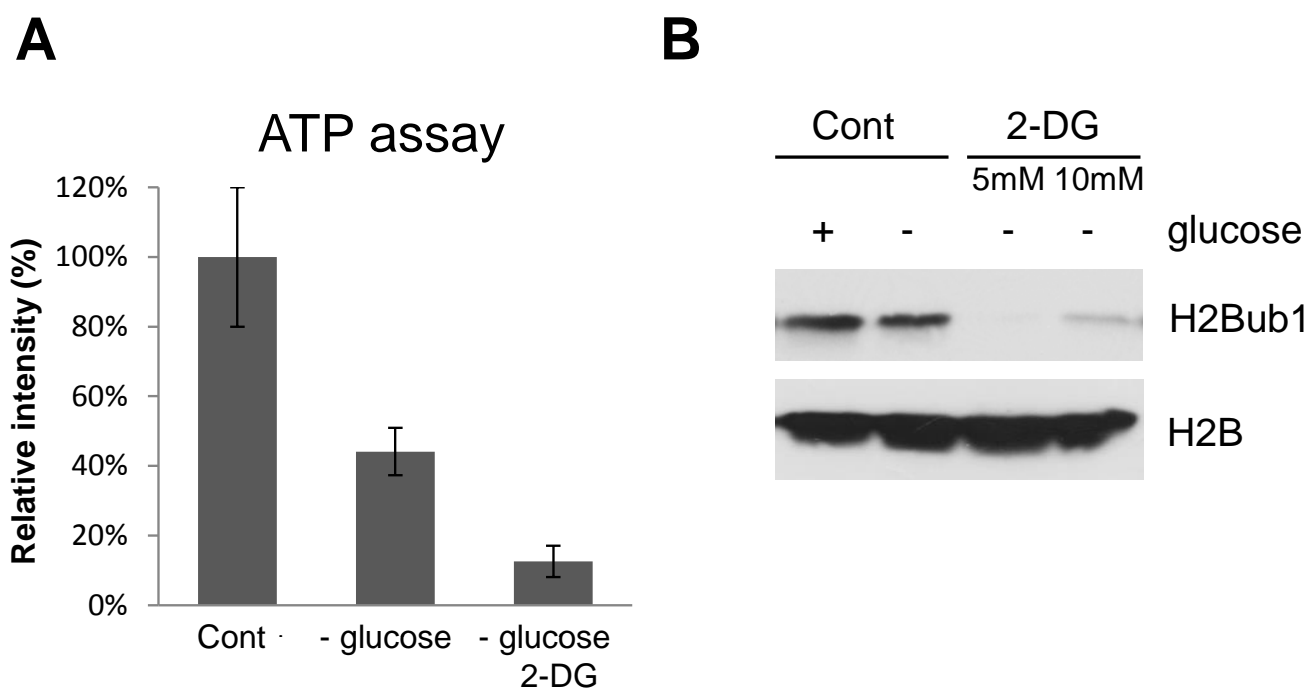

Fig. 25: The glucose metabolism is involved in the regulation of the H2Bub1 level. (A) Detection of ATP using the ATP determination kit. Each reaction contained $1.25 \mu \mathrm{g} / \mathrm{mL}$ of firefly luciferase, 50 $\mu \mathrm{M} D$-luciferin and $1 \mathrm{mM}$ DTT in $1 \mathrm{X}$ Reaction Buffer. After $5 \mathrm{~min}$ incubation, luminescence was measured using a Turner Lumiometer. The amount of ATP in the experimental samples was calculated from the standard curve. (B) MCF10A cell were seeded in a 6-well plate. After $24 \mathrm{~h}$ medium was changed to glucose-free medium and cells were treated with either $5 \mathrm{mM}$ or $10 \mathrm{mM}$ 2-Deoxyglucose for $24 \mathrm{~h}$. Protein extracts were harvested and analyzed by Western blotting for H2Bub1 and $\mathrm{H} 2 \mathrm{~B}$. 
All these observations suggest that the glucose metabolism is involved in the regulation of $\mathrm{H} 2 \mathrm{Bub1}$. This hypothesis is consistent with a previous report showing glucose metabolism is required for glucose-induced monoubiquitination of $\mathrm{H} 2 \mathrm{~B}$ in cultured glioma cells (Gao and Xu, 2011).

\subsection{H2Bub1 levels are reduced due to the activation of a cellular deubiquitinating enzyme}

H2B monoubiquitination is highly dynamic and its level is not only regulated by addition, but also by active removal of the ubiquitin residue from the chromatin. Since the loss of H2Bub1 following cellular stress happens very rapidly, we questioned whether the activity of deubiquitinating enzymes (DUBs) is responsible for the rapid decrease of H2Bub1 levels following cellular stress.

\subsubsection{In vitro deubiquitination assay showed increased deubiquitinating activity upon treatment with stress-inducing factors}

To examine if the loss of H2Bub1 is primarily caused by decreased ubiquitination or whether a specific DUB activity is increased an in vitro deubiqutination assay was performed (Fig. 26A). In order to test this protein extracts from normal cells (substrate) were mixed with extracts from cells where RNF20 and RNF40 were depleted with siRNA to minimize the level of H2Bub1 (effector). When the AICAR- or Doxorubicin-treated cells were used for the effector extract the amount of H2Bub1 was unaffected after 5 min of incubation but after 15 min a decreased H2Bub1 protein level compared to the non-treated control effector cells was observed, indicating that an increased deubiquitinating activity was present in treated cells (Fig. 26B). 
A

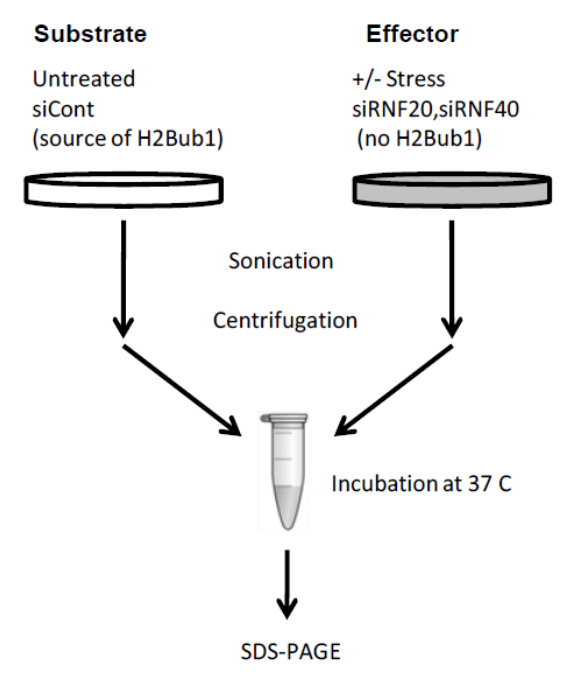

B

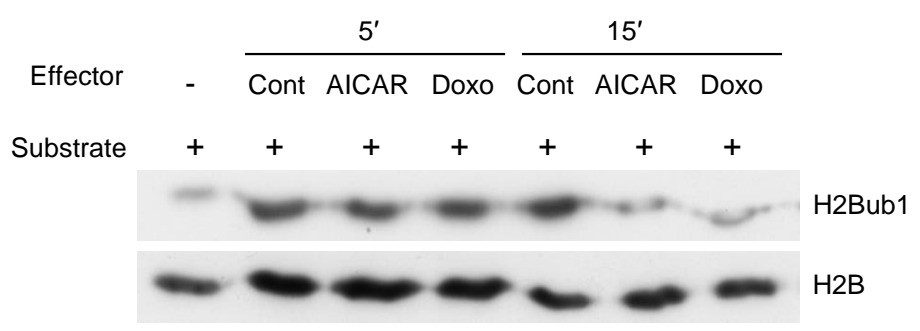

Fig. 26: An H2B deubiquitinating activity is increased during cellular stress. (A) Scheme of the in vitro deubiquitination experiment (Shchebet, 2011). For details see "Materials and methods". (B) MCF10A cells were transfected with control, RNF20 or RNF40 siRNA and treated with $7.5 \mu \mathrm{M}$ Doxorubicin for $3.5 \mathrm{~h}$ or $2.5 \mathrm{mM}$ AICAR for $30 \mathrm{~min}$ prior harvesting and performing in vitro deubiquitination assay. Protein lysates were analyzed by Western blotting with antibodies specific for $\mathrm{H} 2 \mathrm{Bub} 1$ and H2B, the latter used as a loading control.

In order to prove that the loss of ubiquitination caused by the presence of the AICARor Doxorubicin-induced effector extract was provided by DUBs, we performed another in vitro deubiquitination experiment. Therefore co-immunoprecipitations (CoIP) with ATXN7L3 or USP22 overexpression constructs were performed but the subsequent in vitro deubiquitination assay with synthesized nucleosomes did not provide conclusive data (data not shown).

\subsubsection{The SAGA complex is required for H2B deubiquitination}

In mammalian cells the deubiquitinating enzyme module (DUBm) for H2Bub1 is incorporated into the SAGA complex that also exhibits histone acetylation. USP22 (ubiquitin specific protein) is a deubiquitinating enzyme that has been shown to deubiquitinate nucleosomes containing H2Bub1 in vitro. Recently, a report showed that USP22 is allosterically regulated by interactions with different domains of other DUB module subunits, namely ATXN7, ATXN7L3, and ENY2. Consistently, knockdown of the core DUBm component ATXN7L3 led to a significant increase in H2Bub1 (Lang, 2011). To study the importance of deubiquitination in stress-induced 
loss of H2Bub1 the effect of depletion of the SAGA DUBm complex components USP22, ATXN7L3, ATXN7 and ENY2 was analyzed in MCF10 cells.

\subsubsection{USP22 is needed for the stress-induced deubiquitination}

We hypothesized that the activity of USP22 could be crucial for the stress-induced reduction of $\mathrm{H} 2 \mathrm{Bub1}$. As shown in figure 23A, Actinomycin $\mathrm{D}$ treatment leads to reduced global H2Bub1 levels. Western blot analysis revealed that siRNA-mediated knockdown of USP22 in MCF10A cells increases H2Bub1 levels upon Actinomycin D treatment compared to control-siRNA transfected Actinomycin D treated cells.

qRT-PCR experiments were performed to determine whether USP22 knockdown is capable of rescuing the effects of Actinomycin D treatment on RNF20/RNF40dependent genes. Indeed siRNA-mediated knockdown of the deubiquitinating enzyme USP22 restore the decreased gene expression level of the target genes upon Actinomycin D treatment (Fig. 27B). 
A

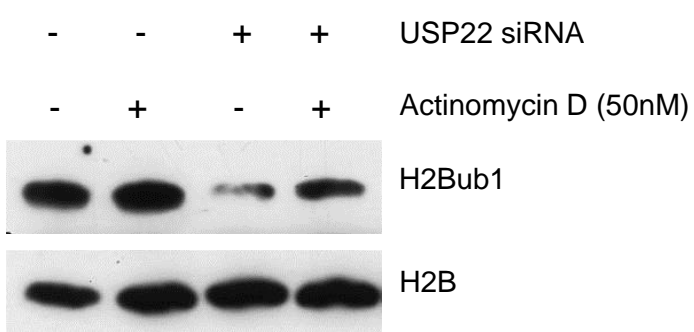

B

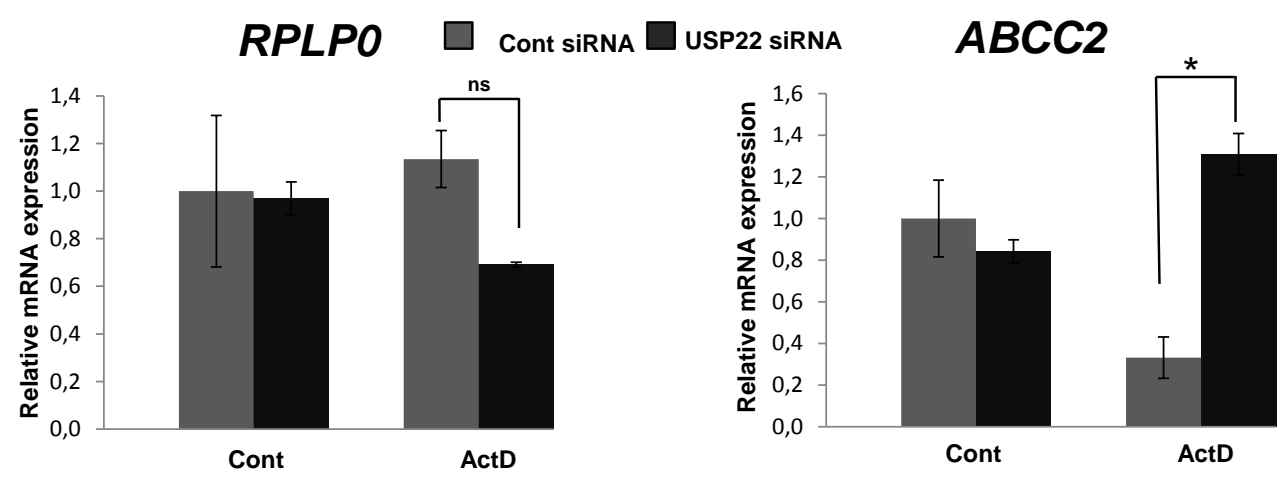

FST
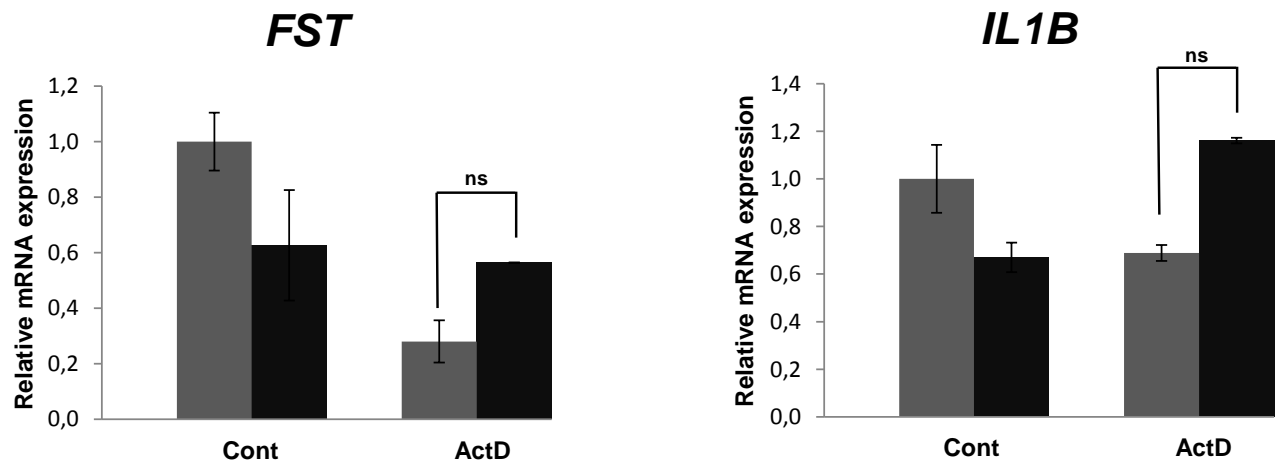

Fig. 27: USP22 regulates the level of H2Bub1. (A) MCF10A cells were transfected with siRNA against USP22. After $72 \mathrm{~h}$ cells were treated with $20 \mathrm{nM}$ of Actinomycin D for $3 \mathrm{~h}$. Extracts were analyzed by Western blot. (B) MCF10 cells were reverse-transfected with 30 pmol control or USP22 siRNA for $72 \mathrm{~h}$ and treated with Actinomycin D for $3 \mathrm{~h}$. Isolated RNA was reverse-transcribed with random primers and analyzed by qPCR. Target gene expression levels were normalized to RPLPO, graphed relative to the control-transfected sample and expressed as "relative mRNA expression". Statistically significant differences are indicated: ${ }^{*} P \leq 0.05 ;{ }^{* *} P \leq 0.01 ;{ }^{* *} P \leq 0.001 ;$ mean $\pm \mathrm{SD}, \mathrm{n}=3$.

To further determine if these effects could be reproduced via Cisplatin treatment, Western blot analyses were performed with different concentrations of the DNAdamaging drug. As shown in figure 24 siRNA targeted to USP22 efficiently rescues H2Bub in both MCF10A (Fig. 28A) and H1299 (Fig. 28B) cells at various concentrations. However, at high concentrations such as $50 \mu \mathrm{M}$ Cisplatin the loss of H2Bub1 can be rescued only partially. 


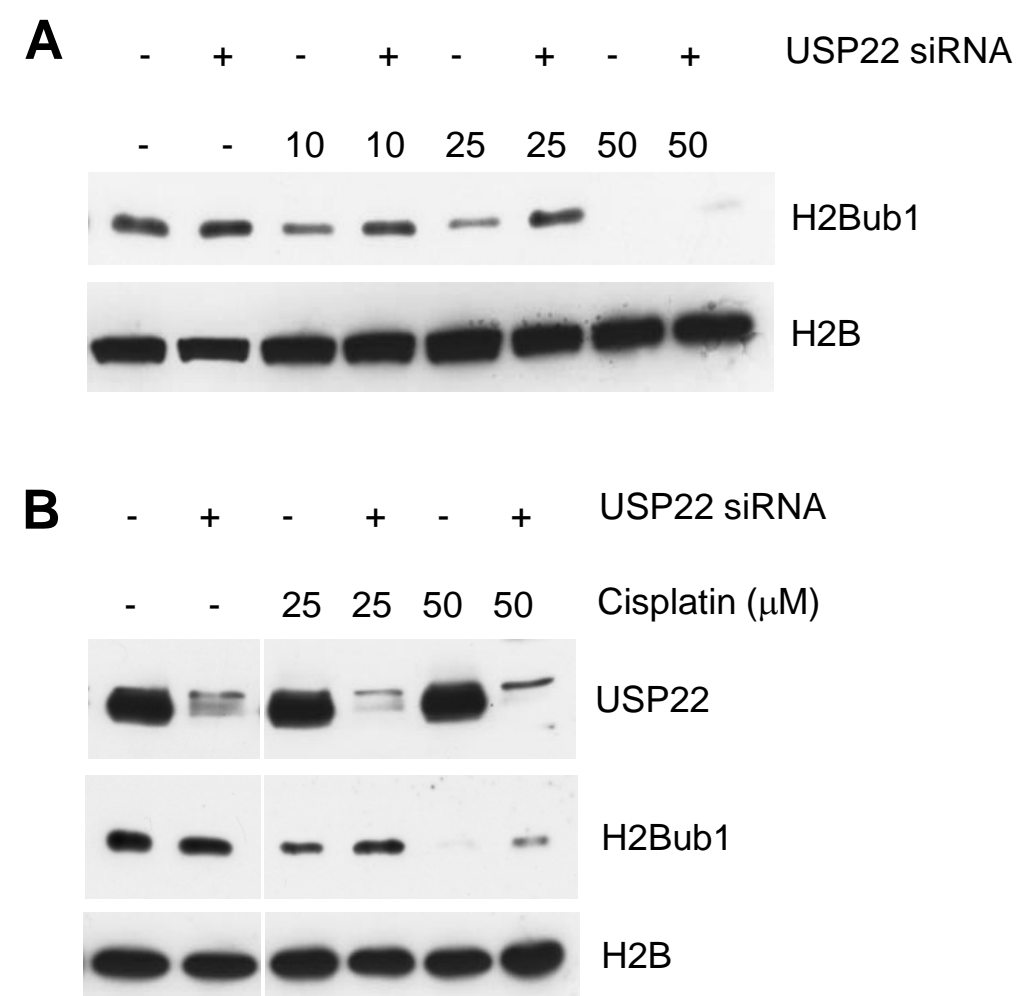

Fig. 28: USP22 is needed for Cisplatin-mediated reduction of H2Bub1. MCF10A (A) and H1299 (B) cells were transfected with siRNA against USP22. After $60 \mathrm{~h}$ cells were treated with indicated concentrations Cisplatin for $12 \mathrm{~h}$. Protein extracts were analyzed by Western blot using the indicated antibodies.

Gene expression studies shown in figure 29 demonstrated again a decrease in $A B C C 2, F S T$ and IL1B mRNA level upon Cisplatin treatment. The mRNA expression levels of the RNF20/RNF40-dependent genes upon USP22 depletion in the presence of Cisplatin were elevated compared to the non-transfected Cisplatin treated control but only $A B C C 2$ showed a significant increase in gene expression level which was comparable to the non-transfected control. 


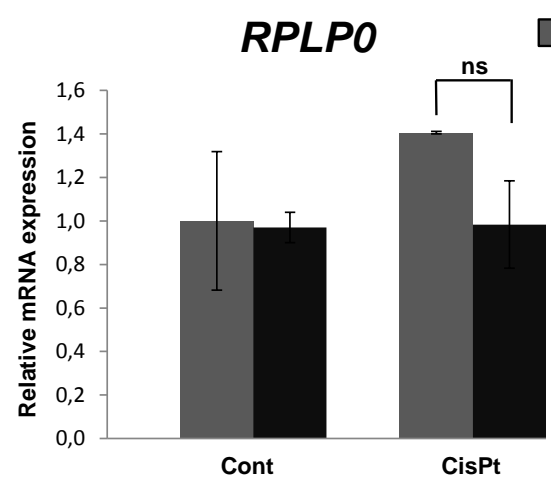

FST

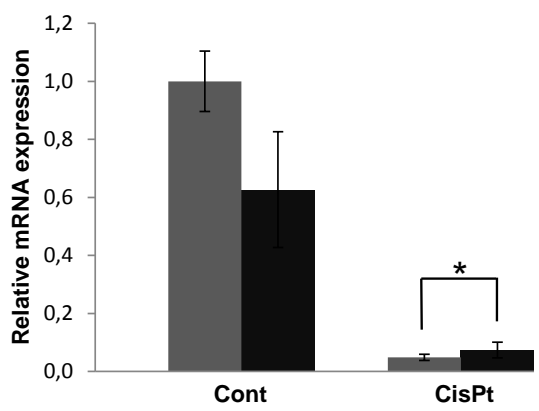

Cont siRNA

USP22 SiRNA

\section{$A B C C 2$}

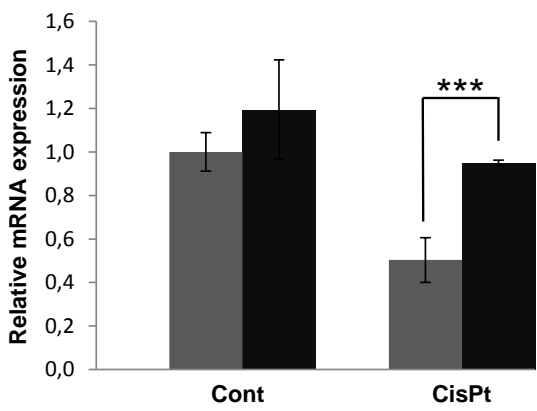

IL1B

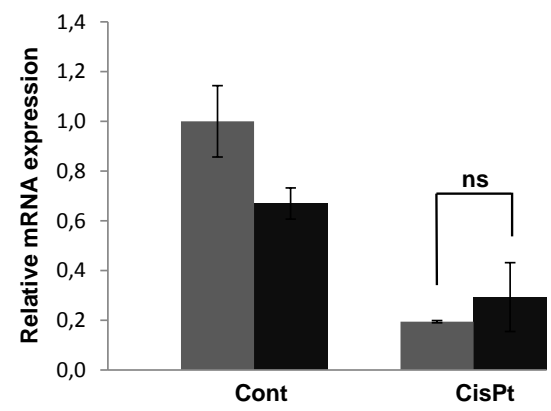

Fig. 29: RNF20 and RNF40 target gene expression upon Cisplatin treatment and USP22 knockdown. MCF10 cells were reverse-transfected with 30 pmol control or USP22 siRNA for $60 \mathrm{~h}$ and treated with Cisplatin for $12 \mathrm{~h}$. Isolated RNA was reverse-transcribed with random primers and analyzed by qRT-PCR. Target gene expression levels were normalized to RPLPO, graphed relative to the control-transfected sample and expressed as "relative mRNA expression". Statistically significant differences are indicated: ${ }^{*} P \leq 0.05 ;{ }^{* *} P \leq 0.01 ;{ }^{* * *} P \leq 0.001$; mean $\pm \mathrm{SD}, \mathrm{n}=3$.

In order to investigate a possible genome-wide role for SAGA deubiquitination activity on H2Bub1 the effect of siRNA-mediated knockdown of USP22 on the H2Bub1 occupancy in MCF10A cells was analyzed by ChIP experiments. ChIP analysis revealed that H2Bub1 levels are general lower at the TSS compared to the TR region. In fact, our data showed that when SAGA deubiquitination activity is impaired by USP22 knockdown the H2Bub1 levels in MCF10A cells were strikingly increased in the three RNF20/RNF40-dependent genes and the control genes (Fig. 30). Importantly, the results of this ChIP analysis confirm the observed rescue of the H2Bub1 levels in Western blot and mRNA gene expression for all tested genes, since a significant increase in H2Bub1 occupancy was observed in Cisplatin treated USP22 knockdown cells compared to Cisplatin treated control siRNA-transfected cells. 
These results imply that USP22 DUB activity is a major regulator of this modification and cannot be fully compensated by other deubiquitinases.
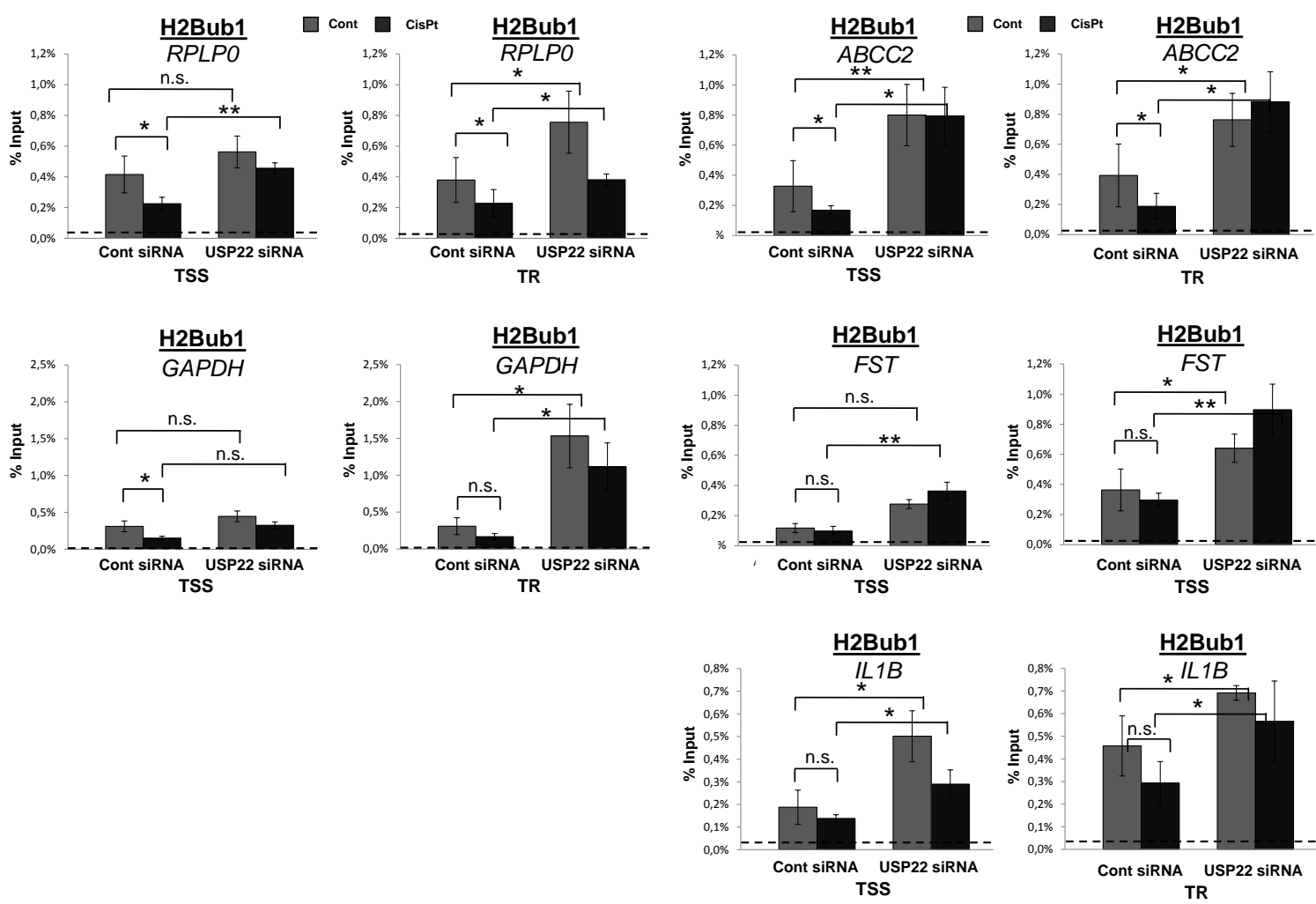

Fig. 30: Regulation of RNF20 and RNF40 target gene expression correlates with H2Bub1 occupancy upon Cisplatin treatment and USP22 knockdown. MCF10 cells were treated as described in Fig. 25. Chromatin extracts were analyzed by ChIP for presence of H2Bub1. IgG antibody was used to determine background binding (indicated as dotted line). H2Bub1 occupancy was normalized to input (indicated as "\% of input"). Statistically significant differences are indicated: ${ }^{*} P \leq$ $0.05 ;{ }^{* \star} P \leq 0.01 ;{ }^{* \star *} P \leq 0.001 ;$ mean $\pm \mathrm{SD}, \mathrm{n}=3$.

\subsubsection{Chemotherapeutical treatment also affects H2A ubiquitination}

Several studies indicated that in addition to $\mathrm{H} 2 \mathrm{~B}$, USP22 deubiquitinates $\mathrm{H} 2 \mathrm{~A}$ in vitro (Zhao et al., 2008). Therefore, we sought to determine if the DNA-damaging drug Cisplatin has a similar effect on H2Aub1 levels. Indeed, treatment with Cisplatin resulted in a decrease in the H2Aub1 protein level which is comparable to that of H2Bub1 as depicted in figure 31. Nevertheless, USP22 knockdown did not appreciably rescue the decreased H2Aub1 level upon Cisplatin treatment compared 
to control siRNA transfected Cisplatin treated cells indicating that activity of USP22 is not crucial for the stress-induced reduction of H2Aub1 levels.

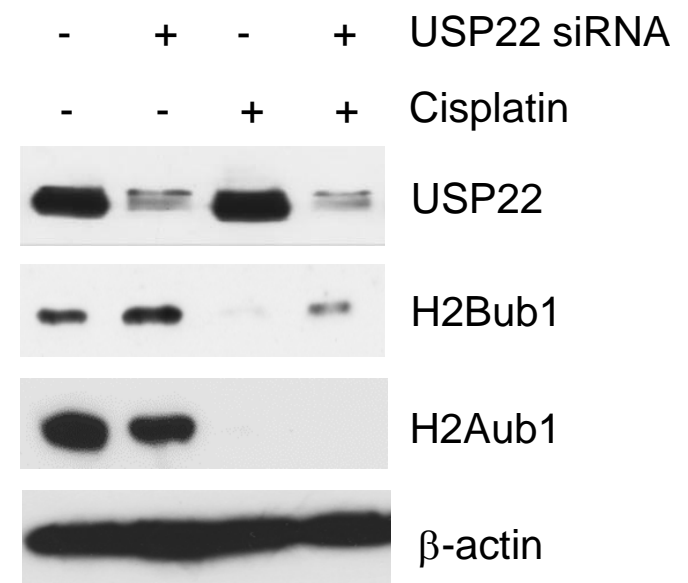

Fig. 31: Cisplatin treatment affects H2A ubiquitination. MCF10A cells were transfected with siRNA against USP22. After $60 \mathrm{~h}$ cells were treated with indicated concentrations of Cisplatin for $12 \mathrm{~h}$. Protein extracts were analyzed by Western blot using H2Aub1 and H2Bub1 antibodies. The efficient knockdown is shown by immunoblotting using USP22 antibody and $\beta$-actin antibody was used as a control.

\subsubsection{Depletion of SAGA DUB module components leads to H2Bub1 accumulation}

To test whether depletion of SAGA DUBm components leads to increased H2Bub1 levels following the induction of cell stress MCF10A cells were transfected with siRNA to ATXN7L3, ATXN7 and ENY2 for $72 \mathrm{~h}$. Western blot analysis confirmed a decrease in H2Bub1 level upon Cisplatin and AICAR treatment and a potent increase in H2Bub1 upon ATXN7L3 depletion either in control or in treated cells. siRNA mediated knockdown of ATXN7L3 showed the strongest up regulation of H2Bub1 uder both, normal and stress-induced conditions, but ATXN7 and ENY2 knockdown also increased H2Bub1 levels upon knockdown compared to non-transfected control aa well as non-transfected stress-induced cells (Fig. 32). 


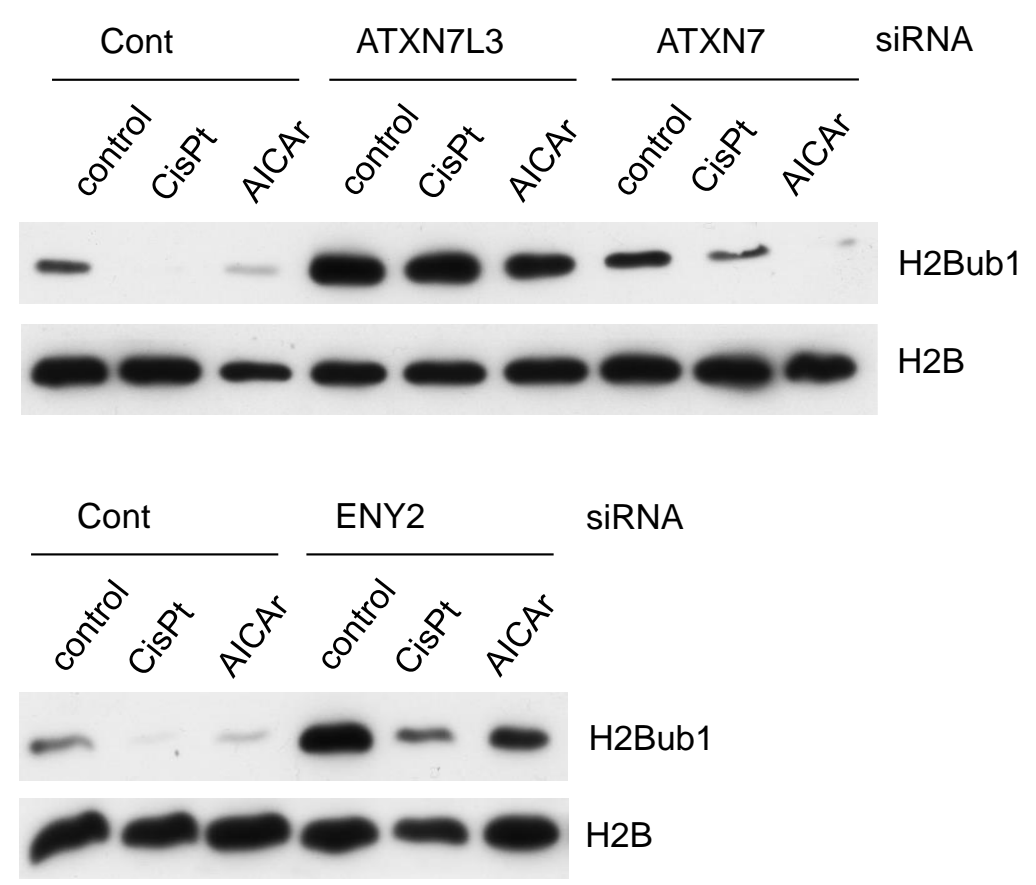

Fig. 32: Knockdown of SAGA DUBm subunits reverses stress-induced loss of H2Bub1 levels. MCF10A cells were reverse-transfected with 30 pmol control, ATXN7L3, ATXN7, and ENY2 siRNA for $48 \mathrm{~h}$ followed by treatment with either $50 \mu \mathrm{M}$ Cisplatin or $2.5 \mathrm{mM}$ AICAR for $30 \mathrm{~min}$. H2Bub1 protein levels were analyzed by Western blot using specific antibodies against H2Bub1 and H2B as a loading control.

To further test this results gene expression analysis upon siRNA-mediated knockdown of ATXN7L3, ENY2 and USP22 was examined in MCF10A cells. Cells were transfected with the indicated siRNAs followed by AICAR treatment for $30 \mathrm{~min}$ and gene expression analyses by qRT-PCR. These data confirm observations from the Western blot. First of all, the efficient knockdown of ATXN7L3, ENY2 and USP22 was confirmed on mRNA levels (Fig. 33). Interestingly, ATXN7L3 knockdown resulted in an increased ENY2 mRNA level but not vice versa indicating that ATXN7L3 expression is possibly crucial for the full activity of the DUBm and depletion of it may need to be compensated by up-regulation of one of its interacting components. For FST gene AICAR decreased mRNA expression levels can be rescued upon ATXN7L3 depletion. During this experiment IL1B do not show any significant changes in gene expression level upon ACIAR treatment or knockdown of any DUBm component. ABCC2 mRNA level is rescued in USP22 depleted MCF10A cells, but not significantly upon ENY2 and ATXN7L3 knockdown. RPLPO was used as a control and was unaffected upon treatment and siRNA-mediated knockdown. 


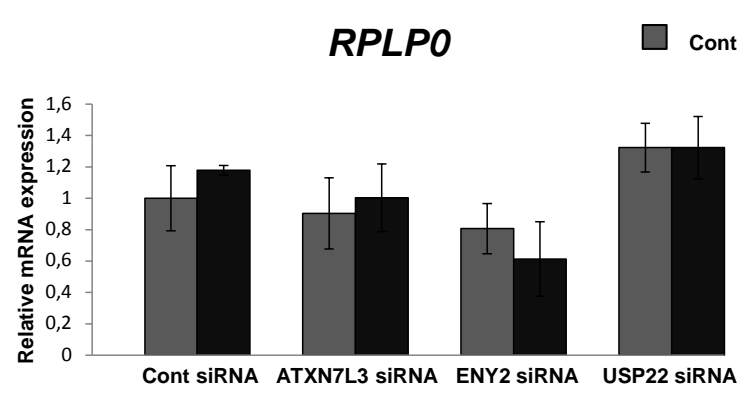

AICAR ENY2

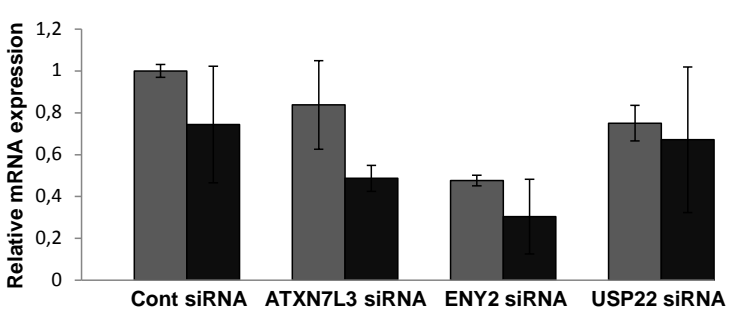

ATXN7L3

USP22
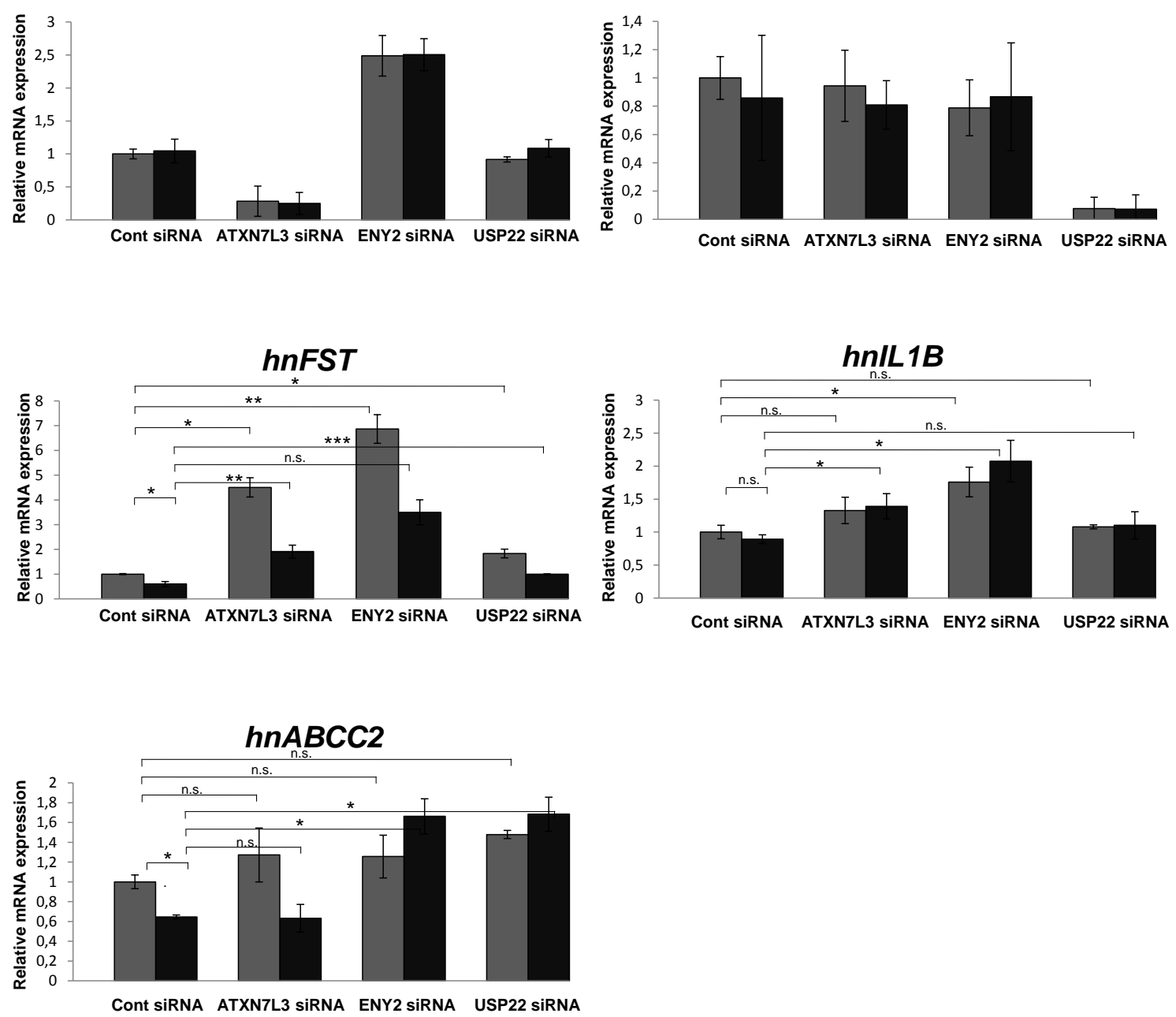

Fig. 33: DUB module components are needed for AICAR-mediated rescue of H2Bub1. MCF10A cells were transfected with siRNA against ATXN7L3, ENY2 and USP22. After $72 \mathrm{~h}$ cells were treated with $2.5 \mathrm{mM}$ AICAR for $30 \mathrm{~min}$. RNA was isolated and CDNA was analysed by qRT-PCR using $\mathrm{hn}$ primers. Gene expression was normalized to $\mathrm{hnRPLPO}$ expression (indicated as "relative mRNA expression"). hn - heterogeneous nuclear. Statistically significant differences are indicated: ${ }^{*} P \leq 0.05$; ${ }^{* \star} P \leq 0.01 ;{ }^{* * \star} P \leq 0.001 ;$ mean values $\pm \mathrm{SD}, \mathrm{n}=3$. 
To check whether H2Bub1 is also increased on RNF20 and RNF40 dependent genes its occupancy was examined by ChIP with corresponding antibodies (Fig. 34). Both RNF20 and RNF40 regulated genes, $A B C C 2$ and FST, demonstrated decreased H2Bub1 occupancy upon AICAR treatment on TSS and TR and an increased H2Bub1 occupancy on both regions upon ATXN7L3 depletion. Interestingly, RPLPO, used as a RNF20 and RNF40 independent gene, also possessed lower H2Bub1 levels in AICAR-treated MCF10A cell and high levels of H2Bub1 after ATXN7L3 knockdown.
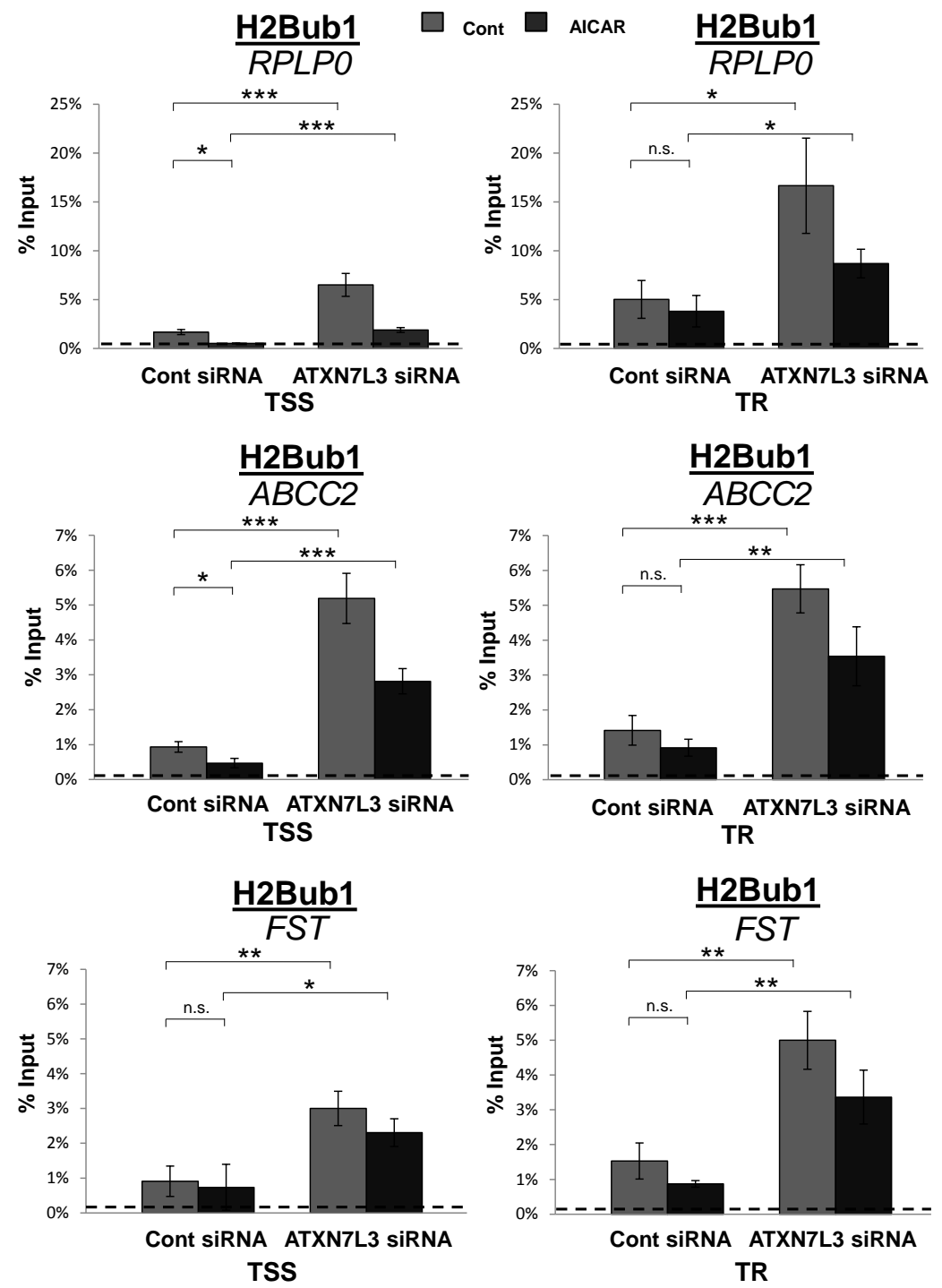

Fig. 34: Correlation of RNF20 and RNF40 target gene expression and H2Bub1 occupancy upon AICAR treatment and ATXN7L3 knockdown. MCF10A cells were transfected with siRNA to ATXN7L3 for $72 \mathrm{~h}$ and treated with AICAR for $30 \mathrm{~min}$. Chromatin extracts were analyzed by ChIP for presence of H2Bub1. IgG antibody was used to determine background binding (indicated as dotted line). H2Bub1 occupancy was normalized to input (indicated as "\% of input"). Statistically significant differences are indicated: ${ }^{\star} P \leq 0.05 ;{ }^{* *} P \leq 0.01 ;{ }^{* * *} P \leq 0.001$; mean $\pm \mathrm{SD}, \mathrm{n}=3$. 
This observation suggests that induction of stress leads to a genome-wide H2Bub1 reduction and inactivation of the SAGA DUB module component results in an accumulation of H2Bub1. Thus, H2Bub1 occupancy in AICAR treated ATXN7L3 depleted cells compared to control siRNA- and AICAR- treated cells was significantly increased on all examined genes.
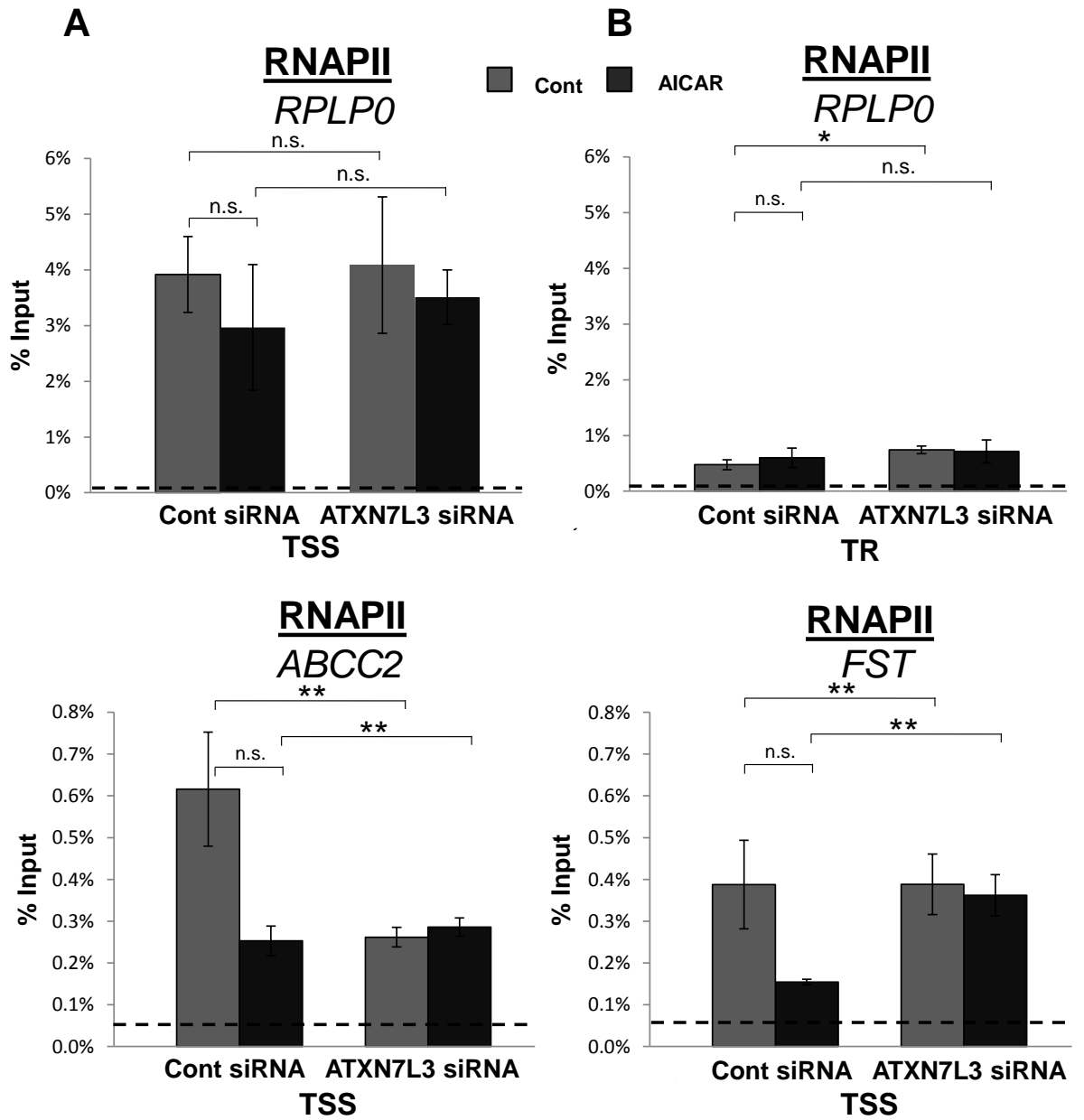

Fig. 35: RNAPII recruitment upon AICAR treatment and ATXN7L3 knockdown. MCF10A cells were transfected with siRNA to ATXN7L3 for $72 \mathrm{~h}$ and treated with AICAR for $30 \mathrm{~min}$. Chromatin extracts were analyzed by ChIP for presence of RNAPII. IgG antibody was used to determine background binding (indicated as dotted line). RNAPII occupancy was normalized to input (indicated as "\% of input"). Statistically significant differences are indicated: ${ }^{\star} P \leq 0.05 ;{ }^{* \star} P \leq 0.01 ;{ }^{* \star *} P \leq 0.001$; mean $\pm S D, n=3$.

H2Bub1 is associated with the transcribed regions of active genes and may promote active transcription. We carried out ChIP analyses with an RNAPII antibody, which recognizes both the phosphorylated and unphosphorylated forms of the RNAPII and thus has been used as a marker for active RNAPII, to test whether ATXN7L3 
knockdown affect the recruitment of RNAPII to RNF20 and RNF40 target genes. ATXN7L3 knockdown did not appreciably affect elongation at the transcribed region of RPLPO. As shown in figure 35A AICAR treatment leads to decreased elongating RNAPII levels at the transcriptional start site. The amount of total RNAPII at the TSS of $F S T$ and $A B C C 2$ was decreased to less than 50\% upon AICAR treatment. ATXN7L3 depletion led to increased recruitment of RNAPII independently of AICAR treatment for FST (Fig. 35B). The results confirm the observed rescue upon ATXN7L3 knockdown and AICAR treatment on FST gene expression level (Fig. 34) and suggest that up-regulated H2Bub1 levels upon ATXN7L3 knockdown facilitate transcriptional elongation even in the presence of stress-inducing factors. For $A B C C 2$ the amount of elongating RNAPII in ATXN7L3 depleted cells in the presence or absence of AICAR do not change compared to AICAR treated control-transfected cells. This could be because $A B C C 2$ gene is unable to rescue AICAR-mediated loss of H2Bub1, since gene expression analysis showed the same result (data not shown).

\subsection{Genome-wide analysis of H2Bub1 distribution}

To better understand H2Bub1 function we carried out genome-wide analysis of H2Bub1 distribution in MCF10A cells. Chromatin immunoprecipitation followed by DNA sequencing (ChIP-Seq) allows genome-wide discovery of protein-DNA interactions and provides insight into the function of histone modifications and chromatin structure (Park, 2009; Robertson et al., 2007).

\subsubsection{ChIP-seq analyses show H2Bub1 occupancy and its changes upon ATXN7L3 knockdown}

Histone $\mathrm{H} 3$ lysine 4 monomethylation (H3K4me) and trimethylation ( $\mathrm{H} 3 \mathrm{~K} 4 \mathrm{me} 3$ ) as well as hyperacetylation of histone $\mathrm{H} 3$ and $\mathrm{H} 4$ are known as active marks and are associated with transcriptional initiation and elongation (Heintzman and Ren, 2009; Wang et al., 2008). Thus, downloaded and analyzed data from the public database European Nucleotide Archive against H3K4me1, H3K9/14ac and H3K4me3 in MCF10A cells gave us a better idea of whether the genes are or can be expressed 
and distribution profiles can be compared with our data of H2Bub1. To analyze our data the sample duplicates for H2Bub1 were merged after mapping and then WIG files which contain a continuous ChIP enrichment signal were identified by the peakcaller MACS (Model-based Analysis of ChIP-seq) using normalization to input (Zhang et al., 2008b). In our ChIP-seq experiment we used a highly specific antibody recognizing H2Bub1 and confirmed that H2Bub1 is mostly associated with the transcribed region of expressed genes. Consistent with other recently published papers, H2Bub1 demonstrated relatively strong signals near the 5' regions of many actively transcribed genes (Jung et al., 2012; Minsky et al., 2008; Vethantham et al., 2012). Data for H3K4me3 and H3ac correlate quantitatively and showed an enrichment in the regions surrounding the transcriptional start sites (TSS) especially downstream as previously reported in human cells (Barski et al., 2007; Jung et al., 2012; Wang et al., 2008).

Up to date, the genome-wide recruitment of the SAGA DUBm and the sites where SAGA deubiquitinates H2B have not been established. ChIP-seq data confirmed the observed increase in H2Bub1 following ATXN7L3 knockdown but the H2Bub1 enrichment does occur uniform across genes, but rather at specific sites primarily towards the 5 ' end. Figure 36A shows the housekeeping genes GAPDH and ACTB which are expressed at relatively constant levels in most situations and HNRNPK, a major pre-mRNA-binding protein that was unaffected in our microarray of RNF20 and RNF40 depleted MCF10A cells and was not regulated upon 17B-Estradiol treatment or following proteasome inhibition in MCF7 breast cancer cells (Prenzel et al., 2011). 


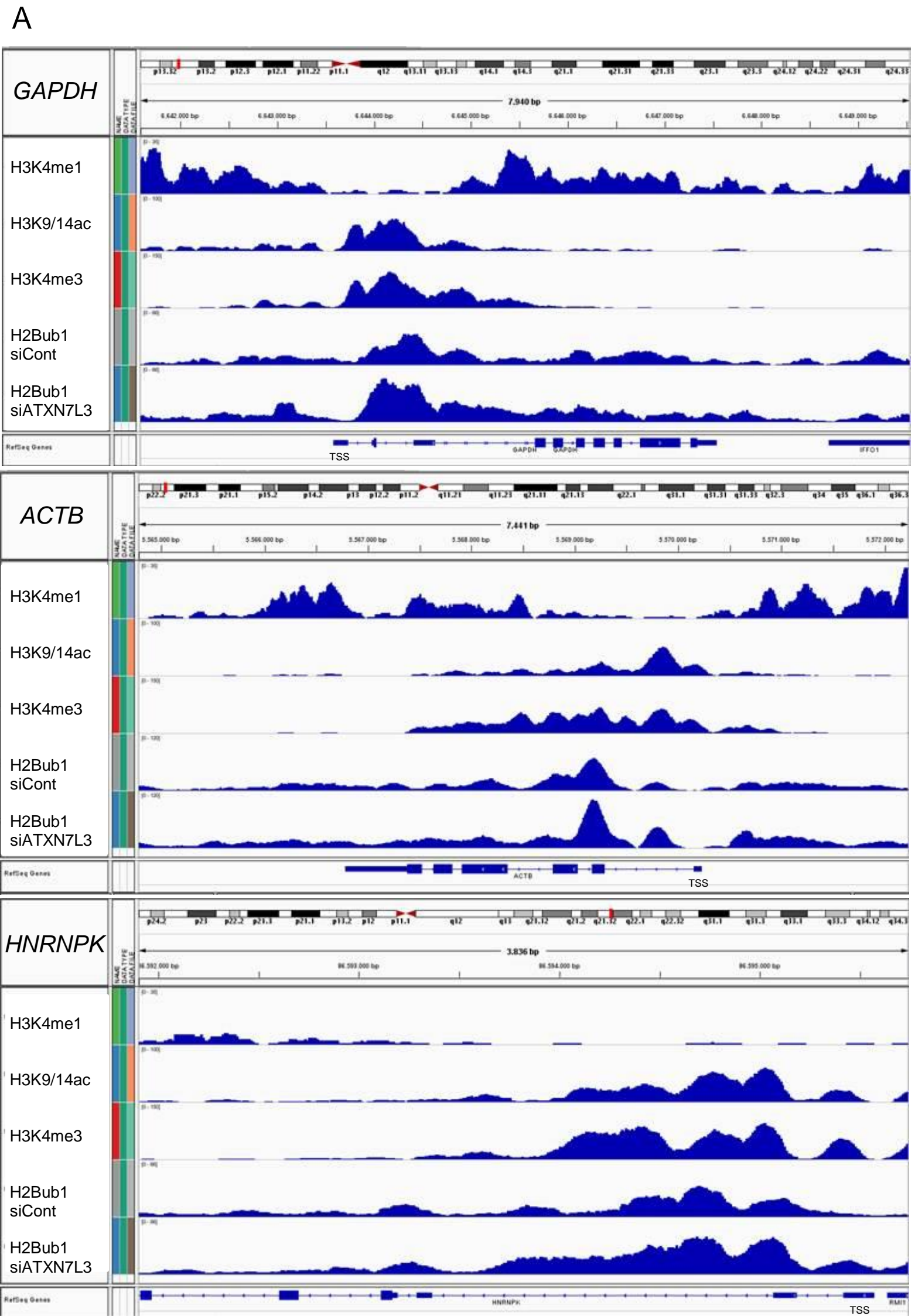


B

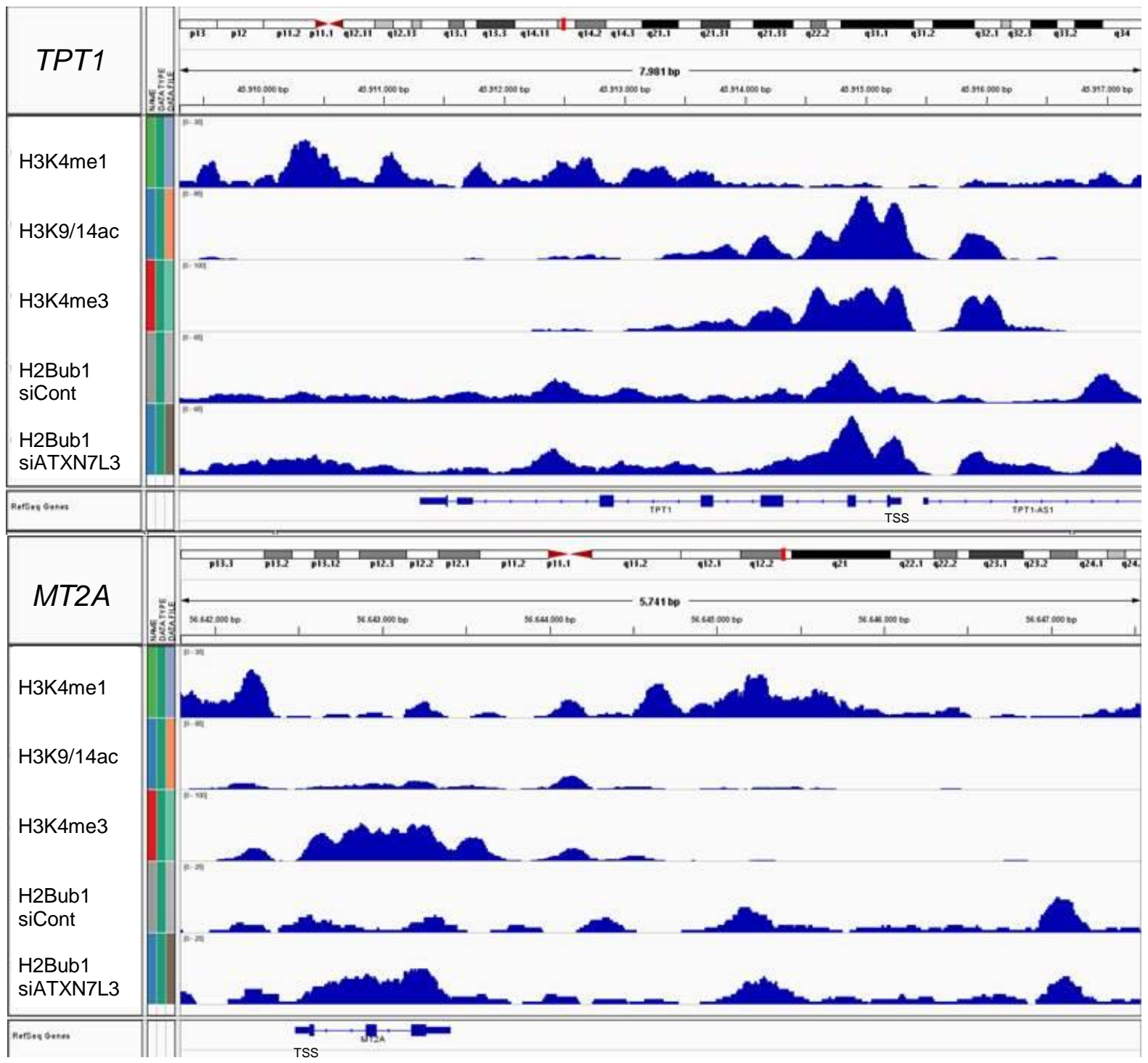

Fig. 36: ChIP-seq profile of different histone marks. Genome browser tracks depicting H3K4me1, $\mathrm{H} 3 \mathrm{~K} 9 / 14 \mathrm{ac}, \mathrm{H} 3 \mathrm{~K} 4 \mathrm{me} 3$ in control MCF10A cells as well as H2Bub1 of control- and ATXN7L3-siRNA transfected MCF10A cells ChIP-seq peaks at representative gene loci. (A) Control genes which are usually highly expressed in cells. (B) Representative data of very highly expressed genes in MCF10A according to our microarray data.

However, generally our data demonstrated an increase in H2Bub1 level upon ATXN7L3 knockdown but not across the whole gene body. For example, on the GAPDH gene there is a significant increase on the second exon. The ACTB gene looks very similar where exon two again shows nearly a doubling of H2Bub1 in ATXN7L3 depleted MCF10A cells and the peak upstream of that also increases at least two fold. On HNRNPK a similar situation occurs since the most significant 
increase is on the second intron. The regions after that show some changes as well, but not nearly as much as close to the 5' end of the gene. Furthermore a new peak from around the 5' end was visible too. Two very highly expressed genes according to our microarray data are depicted in figure 36B. For TPT1 the peak near the 5' end is nearly non-existent until ATXN7L3 knockdown. On MT2A the effects is also clear. This gene is quite small though, so that the increase on the second and third introns is also still fairly TSS proximal and overlaps significantly with H3K4me3. Interestingly, the two peaks in the right part of the figure do not go up, but rather down following ATXN7L3 knockdown, again pointing to the specificity of the effect for the position on the given gene. In most cases a significant change in H2Bub1 levels upon ATXN7L3 knockdown appears to be at the 5' end of the gene. The results of our ChIP data suggest a gene-specific role for SAGA deubiquitination activity on H2Bub1 since ATXN7L3 knockdown increases H2Bub1 levels in the transcribed region of expressed genes.

All together these data reveal that H2Bub1 does not cover the whole transcribed region as previously thought, and the increase of H2Bub1 following ATXN7L3 knockdown is rather visible at specific locations in a gene dependent manner.

\subsubsection{Genome-wide averages of the H2Bub1 profile based on gene expression level}

In order to visualize signal enrichment around the transcriptional start site (TSS), in the gene body and at the transcription terminating site (TTS) we generated average ChIP signal profiles of H2Bub1 in control and ATXN7L3 depleted MCF10A cells around these genomic features. The metagene profiles were plotted using CEAS (Cis-regulatory Element Annotation System), a tool designed to characterize genome-wide protein-DNA interaction patterns from ChIP-chip and ChIP-seq (Ji et al., 2006). The genes were sorted into four quartiles according to gene expression level in control MCF10A cells (data obtained by our microarray). Generation of four sub-groups, $1^{\text {st }}$ quartile (highest expression level), the $2^{\text {nd }}$ quartile (medium to highly expressed), the $3^{\text {rd }}$ quartile (medium to lowly expressed) and the $4^{\text {th }}$ quartile (lowly or not expressed) of the expressed genes allows us to compare between the gene groups. 


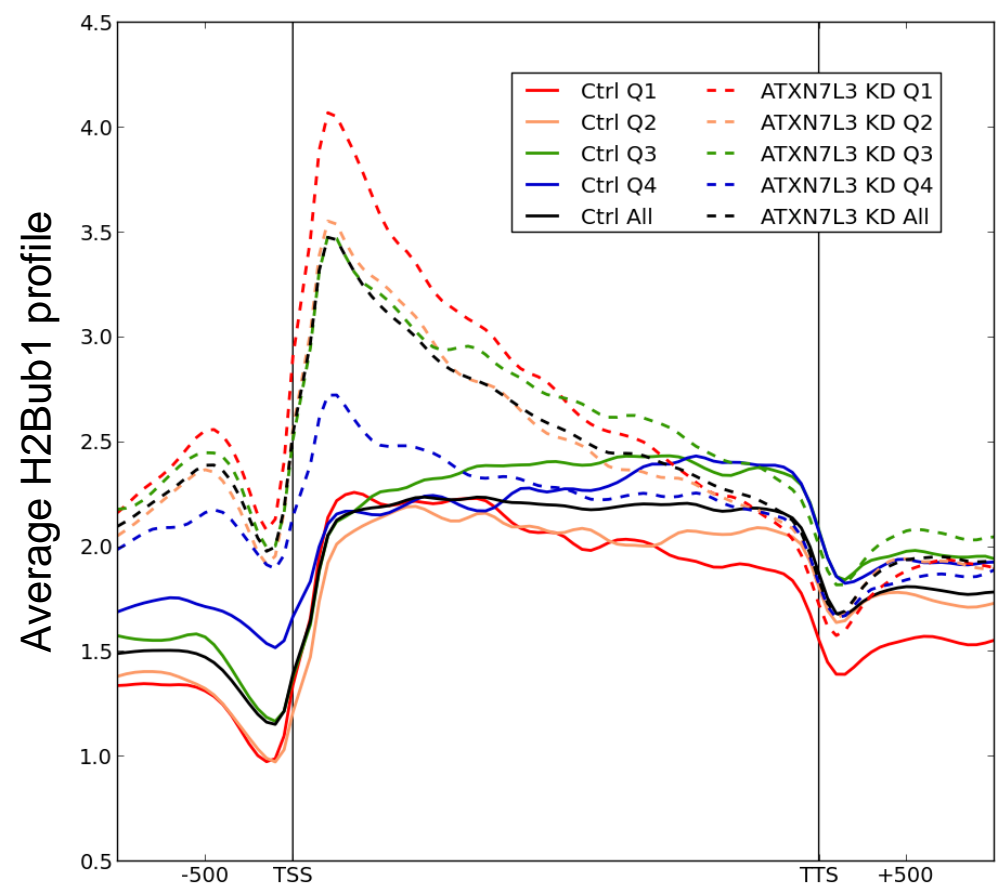

Fig. 37: Average ChIP enrichment signal around TSS, in gene body and around TTS of genes. The red (Q1), pink (Q2), green (Q3) and blue (Q4) colors indicate the average ChIP enrichment signals of the $1^{\text {st }}$ quartile (highest expression level), the $2^{\text {nd }}$ quartile (medium to highly expressed), the $3^{\text {rd }}$ quartile (medium to lowly expressed) and the $4^{\text {th }}$ quartile (lowly or not expressed) of the expressed genes, and black displays all RefSeq genes. The solid lines represent control and the striped lines ATXN7L3 knockdown samples. The average signal is drawn around the transcriptional start site (TSS) and the transcription terminating site (TTS) aligning all 5' ends and all 3 ' ends.

Figure 37 represents the average ChIP signal along the gene with aligned $5^{\prime}$ ends and 3 ' ends and shows that H2Bub1 in control MCF10A cells is low at the TSS, enriched in gene bodies and stays nearly constant towards the 3 ' end where it finally decreases. There is no clear correlation between gene expression level and H2Bub1 profile in control cells. In addition, it was observed that upon ATXN7L3 knockdown H2Bub1 is highly increased downstream of the TSS but is not changed at the TTS compared to control siRNA treated cells. This effect is clearly dependent on the gene expression level. The average ChIP signal of the $1^{\text {st }}$ quartile (Q1) shows the highest increase upon ATXN7L3 knockdown, compared to control cells. The $2^{\text {nd }}$ quartile is lower than the $3^{\text {rd }}$ quartile (Q3) and the $4^{\text {th }}$ quartile (Q4) is lower than the $3^{\text {rd }}$ quartile showing expression-dependent increase following ATXN7L3 knockdown.

Out of these result we can speculate that average H2Bub1 does not necessarily correlate with gene expression but rather that the dynamic ubiquitination and subsequent deubiquitination do correlate with gene activity. 


\section{Discussion}

Chromatin structure and histone modifications have been associated with gene transcription which is tightly regulated by transcription factors and epigenetic factors that control accessibility of the chromatin (Berger, 2007). As more is learned about transcriptional regulation, the ability to gain insight into a cell's response to physiological signals or external stimuli will become increasingly powerful. Genomewide analysis of histone H2B monoubiquitination (H2Bub1) distribution revealed a preferred association with actively transcribed genes in mammalian cells (Minsky et al., 2008). H2Bub1 levels were shown to start near the transcriptional start site with a gradually increase into the transcribed region (Minsky et al., 2008). Recently published data including our results demonstrate a 5' bias for H2Bub1 (Jung et al., 2012; Vethantham et al., 2012). Despite this, little is known about the dynamics of H2B monoubiquitination under different physiological conditions. Our goal was to gain insight into mechanisms of stress-induced H2B deubiquitination and the role of $\mathrm{H} 2 \mathrm{~B}$ monoubiquitination during tumorigenesis and metastasis.

\subsection{The role of $\mathrm{H} 2 \mathrm{Bub} 1$ as a potential tumor suppressor}

\subsubsection{Function of RNF20 and RNF40 in cellular responses}

One way to assess the function of $\mathrm{H} 2 \mathrm{~B}$ monoubiquitination is to deplete or to overexpress the corresponding E3-ligase components and to test the functional consequences. This was analyzed in the current study for the putative ubiquitin ligases RNF20 and RNF40 within a physiological cellular context. The observed antiproliferative effects of siRNA-mediated knockdown of RNF20 or RNF40 on cell cycle progression as well as after RNF40 overexpression suggest that misregulation of the H2Bub1 machinery is tightly linked to cell proliferation. A dominant negative effect could explain the same outcome of RNF40 knockdown as well as its overexpression. For instance unphysiologically high RNF40 overexpression could lead to partial functional inactivation of RNF40 but may not prevent formation of the heterodimer with RNF20. It is known that RNF20 and RNF40 form a complex in vivo and both are required for maintaining H2Bub1 levels in human cells (Kim et al., 2009; Pirngruber et 
al., 2009a). Thus, RNF40 could possibly bear a dominant negative effect on RNF20. When RNF40 as a monomer binds to a different partner and thus prevents the interaction partner to carry out its dimer function, inactivation of this protein complex would be the consequence. For example, RNF40 binds to WAC which is known to recruit the RNF20/RNF40 complex to the chromatin, inhibiting the ability of WAC to bind to its true target and is therefore getting inactive.

Triggered by the growth inhibitory effects of RNF40 knockdown observed in the current study and by the previously published significant increase in cellular migration upon depletion of RNF20 (Shema et al. (2008)), we decided to perform in vitro migration assays and showed increased migratory potential of the normal mammary epithelial cell line MCF10A upon either RNF20 or RNF40 knockdown. Enhanced cell migration is a pronounced feature of advanced tumors and therefore decreased RNF20 and RNF40 expression may potentially facilitate cancer progression. Both cell proliferation and cell migration are crucial to a variety of physiological and pathological processes. The decision of a cell to either proliferate or migrate relies on the balance of many stimulatory and inhibitory control molecules and factors. From in vitro studies it appears that cell proliferation and cell migration are mutually exclusive (Farin et al., 2006; Zheng et al., 2009). The mutual exclusiveness of proliferation and migration may explain why upon RNF20 and RNF40 knockdown the cells display both decreased proliferating capacity and increased migratory potential. Invasive cancer cells can proliferate but may not do so while actively moving. This is consistent with the observation that cells usually stop moving while dividing and then may migrate again. Our data showing growth inhibition and increased migration upon RNF20 and RNF40 depletion raises the question whether inhibition of H2Bub1 would be a benefit or a disadvantage for tumor therapy. Initiation of metastasis is associated with an EMT (extravasation). Carcinoma cells in primary tumors lose cellcell adhesion and enter the bloodstream. To form metastases the circulating tumor cells (CTCs) must invade the target organ (intravasation) and then undergo an MET (mesenchymal-epithelial transition) for clonal outgrowth (Chaffer and Weinberg, 2011). This model suggests that therapeutic inhibition of H2Bub1 should be given constantly because spreading of cells is dispensable if cells stop proliferating. However, intermittent H2Bub1 inhibition may be predicted to enhance tumor 
metastasis. In contrast, our immunohistochemical analyses of H2Bub1 expression in metastases revealed that colonization is H2Bub1-independent since no levels of H2Bub1 could be observed in metastasis tissue microarrays. This suggests that additional pathways may be activated during tumor metastases which overcome a need for H2Bub1 in order to undergo MET. Taken together these findings support the hypothesis that the RNF20/RNF40 complex has a tumor suppressor function probably through its regulation of $\mathrm{H} 2 \mathrm{Bub1}$. This could have interesting implications for therapeutic treatment.

RNF20 suppresses the expression of several proto-oncogenes (Shema et al., 2008). Furthermore, the results of a recent study showed a highly methylated $\mathrm{CpG}$ island in the promoter of the RNF20 gene in human breast tumors in comparison to normal tissues which reduces $R N F 20$ gene expression (Shema et al., 2008) indicating that during tumor progression the expression of the potential tumor suppressor RNF20 may be inhibited by promoter hypermethylation. A question that arises from these observations is whether H2Bub1 accumulation correlates with malignancy. A support for this hypothesis is revealed from our studies on the expression levels of H2Bub1 in primary colon (this study) and breast (Prenzel et al., 2011) tumor samples, which showed a correlation of H2Bub1 loss with tumor progression.

Thus, a loss of H2Bub1 levels during tumor progression may be a potentially therapyrelevant finding. To confirm this hypothesis immunohistochemical studies directly investigating the levels of H2Bub1 in different tumor types and stages, will be important in determining whether loss of H2Bub1 is a general effect occurring during tumor progression. To gain more knowledge on the underlying molecular mechanisms of the function of H2Bub1 and its ubiquitin ligases in cancer, establishment of new conditional mouse knockout models of RNF20 and RNF40 will be necessary to determine the effects of genetic ablation of these genes on tumor initiation and progression in various tumor models. Furthermore, in vitro experiments and in vivo xenograft transplantation experiments may reveal additional insights into the potential tumor suppressor role for H2Bub1. 


\subsubsection{RNF20 and RNF40 modulated gene expression}

In the current study a more global overview of the transcriptional effect upon extensive reduction of H2Bub1 via RNF20 and RNF40 depletion was obtained through mRNA expression profiling in MCF10A cells for a total of 11,223 genes. The microarray analysis revealed that the expression of RNF20 and RNF40, probably through H2Bub1, activates or suppresses distinct gene classes. Consistent with earlier observations in yeast (Mutiu et al., 2007; Tanny et al., 2007) and similar to a recent genome-wide study using RNF20 depleted HeLa cells (Shema et al., 2008), we found that basal expression of only a small subset of genes significantly depends on RNF20 and RNF40.

It is currently unclear why only a subset of genes is modulated by RNF20 and RNF40 knockdown. One reason may be that the heterodimeric RNF20/RNF40 complex affects the activity of only some transcriptional regulators. The search for specific transcription factor motifs located on RNF20/RNF40-regulated genes is one way to examine this possibility. Alternatively, only genes located in particular chromatin domains may be affected by H2Bub1 and thus clusters of adjacent genes could be co-regulated via H2Bub1. Shema et al. (2008) speculated about the function of H2Bub1 in maintaining highly inducible genes in a transcriptionally restricted state. Accordingly, H2Bub1 may prevent accidental expression of these genes in the absence of an activating signal. With its highly dynamic nature H2Bub1 would be predisposed for controlling such rapid switches. The loss of the transcription inhibiting H2Bub1 mark might suppress the expression of these tightly regulated genes. This could be a possible explanation of the more transformed phenotype of cells upon RNF20 and RNF40 depletion in vitro.

Recently published data from our group demonstrated that differentiation-activated genes become inhibited and increase their transcription upon RNF40 depletion suggesting that absence of $\mathrm{H} 2 \mathrm{Bub} 1$ reverses the effect of the differentiation signal. In respect to these findings we hypothesized H2Bub1 to be essential for the rapid and strong transcriptional activation as it happens during differentiation (Karpiuk et al., 2012). Another possible explanation was provided by Fuchs et al. 2012 who revealed that relatively long genes depend more on H2Bub1 in response to differentiation 
signals. Based on lower basal expression levels of RNF20-dependent longer genes one can speculate the requirement of H2Bub1 to switch such genes into a more open chromatin state and thus increases overall transcription elongation rate (Fuchs et al., 2012).

In contrast to the distribution of H2Bub1 on most active genes, suppression of H2B ubiquitination appears to have a mild effect on gene expression. Consistent with earlier observations in yeast (Mutiu et al., 2007; Tanny et al., 2007) and similar to a recent genome-wide study using RNF20 depleted HeLa cells (Shema et al., 2008), we found that only a small subset of genes had an altered expression after depletion of the H2B ubiquitination enzymes in human cells. This raises the question why is only a subset of genes modulated by RNF20 knockdown? H2Bub1 may, instead of directly facilitating transcription elongation could have functions independent of the transcription process itself or alternatively RNF20 and RNF40 may affect the activity of only some transcriptional regulators.

Possible mechanistic explanations for the presumed tumor-suppressor activity of RNF20 and RNF40 can be offered by the identity of RNF20/RNF40 regulated genes. Notably, RNF20 and RNF40 restrain the transcription of genes whose expression correlates with malignancy.

From our microarray we found genes implicated in the IL1B signaling pathway, including $I L-1 \beta$ itself and $I L 1 R A P$. IL-1 $\beta$ is a pleiotropic pro-inflammatory cytokine and is negatively regulated by the ubiquitin ligase complex. $I L-1 \beta$ is closely associated with tumor cell proliferation (Farrow and Evers, 2002; O'Neill, 2000) possibly explaining the anti-proliferative effects of RNF20 and RNF40 depleted cells as documented in this study. $I L-1 \beta$ forms a complex with an Interleukin-1 receptor accessory protein (IL1RAP), which is also repressed upon RNF20 and RNF40 depletion. The data obtained in these studies are contradictory to increased migratory potential of RNF20 and RNF40 depleted MCF10A cells but can be explained by the use of a different signaling pathway or tumor type specificity.

Another tumor-relevant pathway found to be regulated is the activin-signaling pathway. Activin signaling has been involved in many physiological processes, including regulation of cell proliferation, apoptosis and carcinogenesis (Risbridger et 
al., 2001; Woodruff and Mather, 1995). The Follistatin FST gene is a TGF- $\beta$ antagonist found to be down-regulated in an EMT core signature (Taube et al., 2010) and displays an additional interesting RNF20/RNF40 target. Both activin and TGF- $\beta$ have anti-proliferative effects on many types of cells, including cancer cells and function as tumor suppressors in early stage tumorigenesis (Chen et al., 2006; Massagué, 1998). Follistatins mediate cell growth, development, and differentiation in various tissues and can block the interaction between activins and their cell-surface receptors by binding activin and thus suppress activin signaling activity (Welt et al., 2002). Follistatin interacts with the activin A receptor ACVR1C, which is up-regulated in our microarray experiment and thus possibly explains some of the biological effects documented in figures 5, 9 and 13 .

Furthermore, RNF20/RNF40 depletion down-regulates $A B C C 2$ expression (Fig. 12). $A B C C 2$ is a multiple organic anion transporter that transports a variety of anticancer drugs, including Cisplatin (Cui et al., 1999) and Actinomycin D (Lin et al., 2011). The transport of Actinomycin $D$ and Cisplatin by ABCC2 could be therapeutic relevant as it potentially alter drug exposure und influence toxicities (Hill et al., 2013). ABCC2 is expressed in different tumor cell lines (Narasaki et al., 1997) and in some solid tumors (Sandusky et al., 2002). Some Cisplatin- and Doxorubicin-resistant cell lines have been reported to have highly increased ABCC2 mRNA levels (Kool et al., 1997; Taniguchi et al., 1996).

Surprisingly, RNF20/RNF40 depletion elevated the expression of the proto-oncogene MET in MCF10A cells. C-MET encodes a transmembrane receptor tyrosine kinase, that has been involved in tumorigenesis (Naldini et al., 1991). Data of several studies obtained that increased MET levels are associated with metastasis and poor prognosis (Beviglia et al., 1997; Camp et al., 1999; Ghoussoub et al., 1998). The tumorigenic function of MET in vivo remain largely unknown. Overexpressed MET proto-oncogene mediates development of metastatic mammary tumors in transgenic mice (Jeffers et al., 1998; Liang et al., 1996). The results are in good agreement with the observed increase of $M E T$ in our microarray and the increased migratory potential of MCF10A cells upon RNF20/RNF40 depletion. 
While H2Bub1 is required for the expression of some genes, the transcriptional suppression effects observed on other genes may be linked to decreased recruitment of the transcriptional elongation factor TFIIS to certain proto-oncogenes (Shema et al., 2011). The sum of these effects on growth promoting and growth restrictive pathways, as well as RNF20- and RNF40-related alterations in human cancer, suggest a tumor-suppressor role of H2Bub1 and its ubiquitin ligases RNF20 and RNF40 likely through selective modulation of defined subsets of genes by a combination of positive and negative transcriptional or posttranscriptional effects.

\subsubsection{Substrate specificity of RNF20 and RNF40}

At present our DNA microarray data raises the concern whether some of the effects of RNF20 and RNF40 depletion may involve other, unknown targets of its ubiquitin ligase activity. Consistent with this speculation, RNF20 has been shown to target the ErbB3-associated protein Ebp1. Ebp1, a putative tumor suppressor protein possesses two isoforms, $\mathrm{p} 42$ and $\mathrm{p} 48$, that demonstrate different binding affinities to the ErbB3 receptor (Liu et al., 2006). Surprisingly, p42 but not p48 binds to ErbB3 receptor which is strongly stimulated by EGF and inhibits cell proliferation and induces the differentiation of cancer cells. Moreover, RNF20 polyubiquitinates p42 resulting in its degradation in human cancers (Liu et al., 2009). These findings open the possibility that RNF20/Ebp1 interaction may directly influence EGF-signaling. Deficiency of either Ebp1 or RNF20 might lead to increased EGF-signaling and could explain the pronounced effects on the expression of key growth-regulating genes upon RNF20 depletion (Shema et al., 2008). Thus, increased EGF-signaling due to RNF20 depletion and a concomitant decrease in H2Bub1 might explain why specifically EGF-regulated genes are affected despite the fact that H2Bub1 is distributed on most active genes. Thus, these effects may potentially be independent of RNF20 and RNF40 effects on chromatin structure. Similarly to the ubiquitinationdependent loss of Ebp1 in cancer tissues our data show decreased H2Bub1 in colon and breast cancer tissue (Prenzel et al., 2011). However, up to now it is not known, if and how RNF40 affects the Ebp1 activity or ubiquitination.

Staring protein, the rat ortholog of RNF40 was shown to ubiquitinate Syntaxin 1 resulting in its degradation (Chin et al., 2002). Further support for the existence of 
additional RNF20/RNF40 substrates is the composition of the heterodimeric complex itself. Most metazoans and Schizosaccharomyces pombe require an obligate heterodimer for H2B monoubiquitination while Saccharomyces cerevisiae, Drosophila melanogaster and Caenorhabditis elegans only appear to have one Bre1/RNF20/RNF40 ortholog.

Moreover, a series of studies showed that while both RNF20 and RNF40 possess an intact RING-finger domain, only the RING finger of RNF20 was needed for transcription-coupled monoubiquitination of $\mathrm{H} 2 \mathrm{~B}$ in vitro (Kim et al., 2009). Provided the RNF40 RING-finger domain and therefore its ubiquitin ligase activity are not essential for global H2Bub1 levels, why is it then evolutionarily conserved? A plausible explanation is its requirement for different substrates, possibly independent of RNF20. Further protein-protein interaction and mass spectrometry studies are necessary to uncover additional substrates of RNF20 and RNF40 and to reveal additional insight into the molecular mechanism involved in the biological effects of both ubiquitin ligases.

\subsection{Stress-induced H2B deubiquitination}

\subsubsection{H2Bub1 is strongly reduced by various treatments}

In the course of this study we have demonstrated that the ubiquitination of histone $\mathrm{H} 2 \mathrm{~B}$ is rapidly and strongly reduced upon various treatments such as chemotherapeutic drugs (Doxorubicin, Cisplatin), osmotic stress (Sorbitol), heat shock and induction of ribosomal stress by Actinomycin D. The DNA-damaging drug Doxorubicin decreased H2Bub1 levels at much higher concentrations than needed for a DNA-damage response. These findings are not astonishing taken into account that recent reports showed that H2Bub1 is induced at DNA damage sites and is needed for the proper repair (Kari et al., 2011; Moyal et al., 2011; Nakamura et al., 2011). Previous research proposed that the loss of H2Bub1 upon treatment with inhibitors of transcription and heat shock is because of depletion of free monomeric ubiquitin due to insufficient recycling of ubiquitin from polyubiquitinated proteins (Davie and Murphy, 1990). This model cannot be confirmed since most treatments do 
not lead to an accumulation of polyubiquitinated proteins in the cell (Shchebet, 2011) and furthermore, Actinomycin $\mathrm{D}$ was reported to inhibit the accumulation of polyubiquitinated proteins (Ding et al., 2007). Altogether our data show a strong reduction of H2Bub1 upon treatment suggesting the existence of an additional actively regulated multiple factor-induced H2Bub1 regulating pathway upon stress conditions.

The physiological role of this rapid deubiquitination is not yet clear. Yeast cells can rapidly shut off transcription and start the gene expression adapted to the new conditions again within 15 min (Miller et al., 2011). In addition, a recent study assessed the role of H2Bub1 on expression changes during photomorphogenesis that initiates a rapid developmental switch in Arabidopsis thaliana showing the rapid and extensive transcriptional reprogramming (Bourbousse et al., 2012). It can be speculated that H2Bub1 as a highly dynamic transcription-specific chromatin modification may have a similar transcriptional function in human cells.

\subsubsection{JNK and nucleolar signaling are involved in reduction of H2Bub1}

Nucleoli are known to rapidly respond to environmental stress. Stress stimuli can activate members of the c-Jun NH2-terminal protein kinases (JNKs) that balance prosurvival stimuli with opposing signals leading to apoptosis (Schreiber et al. 1995; Chen et al. 1996). JNK inhibits rRNA synthesis in response to stress by inactivating the transcription initiation factor TIF-IA which is required for RNAPI-dependent transcription (Bodem et al., 2000; Moorefield et al., 2000). In turn cells rapidly downregulate the synthesis of ribosomal RNA and release nucleolar proteins to carry out other regulatory functions (Mayer and Grummt, 2005). The results of our study showed a correlation between the loss of H2Bub1 upon Actinomycin D treatment and JNK activation, since both happened in the same range of concentration. What is more, pretreatment with a specific JNK inhibitor which partially rescued the Actinomycin D induced reduction of H2Bub1 levels providing circumstantial evidence for the dependence of the removal of this histone mark on JNK activity (Fig. 20). Based on these data one can speculate that the kinase activity of JNK can downregulate global H2Bub1 levels. 


\subsubsection{Metabolic regulation of H2Bub1 levels}

In addition, we examined whether further kinases are involved in the stress-induced loss of H2Bub1. AMP-activated protein kinase (AMPK) regulates cellular metabolism in response to energy and was reported to activate transcription through direct association with chromatin (Bungard et al., 2010). Furthermore, implication of AMPK in activation of JNK was described (Schulz et al., 2008; Shirwany and Zou, 2010). AICAR is a known activator of AMPK and a previous study showed its ability to induce the phosphorylation of JNK, p38, MAPK, and ERK1/2 (Fisslthaler and Fleming, 2009). The remarkable reduction of global H2Bub1 levels upon AICAR treatment observed in the current study implies a potential role of AMPK in H2Bub1 regulation. However, knockdown experiments using siRNAs directed against AMPK $\alpha 1, A M P K \alpha 2$ and the upstream AMPK-activating kinase LKB1 did not support the hypothesis of AMPK-involvement in the regulation of $\mathrm{H} 2 \mathrm{~B}$ monoubiquitination since little or no rescue of the reduced H2Bub1 level was detectable upon their depletion. Stress conditions deplete ATP stores and increase AMP content in cells (Hardie et al., 1999). AMPK recognizes changes in the AMP/ATP ratio by binding in a competitive manner (Xiao et al., 2007). Thus, increases in the concentration of AMP activate AMPK. Recent findings imply that only a small fraction of the total amount of cellular AMPK is amenable to AMP activation, suggesting that additional regulatory interactions, such as compartmentalization or covalent modifications (e.g. phosphorylation), are also involved in this binding process (Bungard et al., 2010). These data suggest that the loss of H2Bub1 protein level upon treatment of different stress-inducing factors might be less a consequence of activation of AMPK level but more due to changes in AMP/ATP ratio and metabolism itself. A further support for the involvement of metabolism in the regulation of H2Bub1 levels is the remarkable depletion of cellular ATP upon treatment with the glycolysis blocker 2-Deoxy-DGlucose (2-DG) which also results in decreased H2Bub1 levels. Metabolic reprogramming is associated with tumorigenesis and glucose can induce $\mathrm{H} 2 \mathrm{~B}$ monoubiquitination in yeast and human cells (Urasaki et al., 2012). 


\subsection{Activation of a cellular deubiquitinating enzyme reduces H2Bub1 levels}

H2B monoubiquitination is a highly dynamic process and its level is not only regulated by addition, but also by active removal of the ubiquitin residue from the chromatin. According to our data the loss of H2Bub1 happens very quick which cannot be explained by decreased RNF20 and RNF40 levels alone. We hypothesized the activity of deubiquitinating enzymes (DUBs) as a responsible mechanism for the rapid removal of H2B monoubiquitination. Indeed, we demonstrated an increased deubiquitinating activity in Doxorubicin and AICAR treated cells. In our studies, we could observe a rescue of the H2Bub1 levels in Western blot and mRNA gene expression of RNF20/RNF40 target genes upon USP22 and ATXN7L3 depletion.

In mammalian cells the DUB module for H2Bub1 is incorporated into the large SptAda-Gcn5-Acetyltransferase (SAGA) complex. USP22 is the deubiquitinating component of SAGA and was shown to deubiquitinate H2B in vitro (Zhao et al., 2008). USP22 interacts with the DUBm subunits ATXN7, ATXN7L3, and ENY2. Our results allowed us to conclude that USP22 is at least partially responsible for the stress-induced deubiquitination. However, knockdown of core SAGA DUBm components like ATXN7L3 led to a more significant increase in H2Bub1 (Lang et al., 2011).

Thus, possible phosphorylation activity of JNK or different kinases on USP22 may modify the affinity of the USP22 zinc-finger domain to certain interaction partners like ATXN7L3, ENY2 or ATXN7. The release of the complete DUB module from the core SAGA complex may influence the activity of USP22 independent of the rest of the SAGA complex. This hypothesis is consistent with the observation that knockdown of GCN5, a core component of SAGA, did not affect H2Bub1 levels. The USP22 knockdown led to a partial rescue of H2Bub1 upon stress-conditions, suggesting that other ubiquitin hydrolases may also perform deubiquitination of H2Bub1 in addition to USP22. Additional USPs, for example USP27X and USP51 share sequence homology with USP22 and USP27X was shown to interact with it (Sowa et al., 2009). 
As previously mentioned the proteins involved in the regulation of H2Bub1 levels are often misexpressed in cancers. Considering H2Bub1 as a tumor suppressor allows utilizing its regulators as potential therapeutic targets. Changes in the expression or activity of the ubiquitin conjugating enzyme UBE2A, the ubiquitin ligases RNF20 or RNF40 or the deubiquitinase USP22 as well as other subunits of the SAGA DUB module could all potentially play a role during tumorigenesis. USP22 was also discovered as a component of a so-called "death from cancer" 11- gene signature, a specific transcriptional profile of tumor cells containing genes whose expression is associated with poor clinical prognosis of cancer patients (Glinsky, 2005). Thus aberrant expression of USP22 can promote cancer (Liu et al., 2010, 2011; Zhang et al., 2011).

Since our studies revealed a loss of H2Bub1 during tumor progression and metastasis we speculate decreased cellular differentiation and reversion to a stem cell-like phenotype. This hypothesis is supported by the correlation of USP22 mRNA levels with an undifferentiated stem-like phenotype in multiple types of cancer (Glinsky, 2005). Thus, multiple mechanisms which diminish H2B monoubiquitination such as decreased RNF20 or RNF40 expression or increased USP22 expression may similarly lead to a loss of H2Bub1 and reversion to a stem cell-like phenotype. Based on these data one could speculate that H2Bub1 and its upstream regulatory factors may have tumor and stage-dependent functions. An active suppression of H2Bub1 accumulation can be suggested, since several tumor types showed overexpressed USP22 (Glinsky, 2005) or hypermethylation of the RNF20 promoter (Shema et al., 2008). Additional ChIP and ChIP-seq studies to analyze H2Bub1, RNF20/RNF40, USP22 and ATXN7L3 occupancies and their correlation to global gene expression in malignant compared to normal cells may help to determine which benefit H2Bub1 depletion might have.

Our group recently showed that H2Bub1 regulates differentiation of human mesenchymal stem cells (hMSCs) (Karpiuk et al., 2012). The presence of H2Bub1 is required for the resolution of bivalency on differentiation-regulated genes. This study identified a correlation between decreased differentiation and low H2Bub1 levels. In conjunction with the findings, a recent study confirmed H2Bub1 as an important 
regulator of stem cell differentiation and suggest the importance of dynamic changes in H2Bub1 patterns in ESC (embryonic stem cell) differentiation (Fuchs et al., 2012).

In addition to USP22, USP44 was recently reported as an H2B deubiquitinase which is highly expressed and maintains low levels of this modification in ES cells (Fuchs et al., 2012; Zhang et al., 2012). These two DUBs share a similar domain organization with a ZnF-UBP domain which was found in a subclass of 10 human USPs and plays an important role in the regulation of their enzymatic activity. It was shown that the ZnF-UBP domain of USP22 mediates interactions with other SAGA DUBm subunits and is therefore required for USP22 activation (Lang et al., 2011). Bioinformatic analyses predict that the ZnF-UBP from USP44 might be an ubiquitin-binding domain (Bonnet et al., 2008). It can be speculated that this enzyme acts as a sensor of free ubiquitin since it might be activated through the binding of free ubiquitin. Overall, it can be expected that besides SAGA that appears as the major H2B deubiquitination complex on mammals, other DUBs such as USP3, which is the closest homolog of yeast Ubp8 (Nicassio et al., 2007) or USP7 that was described to deubiquitinate H2B in Drosophila and in human cells (van der Knaap et al., 2005, 2010) will have specific effects in given cell types.

\subsection{H2Bub1 as a therapeutic target in cancer}

Given the function of $\mathrm{H} 2 \mathrm{~B}$ monoubiquitination in diverse nuclear processes including transcription and DNA repair, this pathway may be viable for therapeutic treatment. As it was demonstrated in the current study changes in H2Bub1 levels play a role in cancer. In order to investigate the potential role for H2Bub1 as a target for cancer therapy, it is important to determine levels of H2Bub1 in patient tumors and check for correlation with other clinical parameters especially patient outcome and therapeutic responsiveness as well as receptor status in breast cancer, e.g. HER2, estrogen receptor and progesterone receptor.

Down-regulation of H2Bub1 during malignancy is in concordance with the "dedifferentiation" model. For patients with USP22-overexpressing tumors, treatment with USP22 siRNAs or specific deubiquitinating inhibitors are conceivable and could 
be used to reinstate H2Bub1. As a proof of principle, specific DUB inhibitors have already been developed for USP7 and USP8 (Colland, 2010). Treatments with these small-molecule inhibitors might restore a more differentiated cellular phenotype and thereby decrease cell proliferation thus provide a new tool for targeted therapy.

Recent investigation identified a Polycomb signature as a key regulator of a stem cell-like transcriptional profile (Lahad et al., 2005; Ramalho-Santos et al., 2002). Two members of the stem cell signature belong to the Polycomb group of genes (Zhang et al., 2008a). BMI-1 catalyzes ubiquitination of histones and is essential for the function of Polycomb (Ben-Saadon et al., 2006). A proposed model is the importance of simultaneous induction of Polycomb ubiquitin ligases and USP22 during cancer progression. This model is supported by the requirement of USP22 for Myc and the potential contribution of BMI-1 with Myc during transformation (Zhang et al., 2008a). Thus, these findings suggest that overexpressed USP22 in cancer cells may activate the BMI-1 dependent pathway to induce stem cell-like characteristics like therapy resistance and increased metastasis (Xu et al., 2012).

RING-ligases are difficult to target with chemical inhibitors, but recent success in the development of specific inhibitors of the E3 ligase activity MDM2 (murine double minute 2) and the MDM2-p53 interaction (Vassilev et al., 2004; Yang et al., 2005a) indicate the possibility of targets for other E2 and E3 ligases, including H2B monoubiquitination. The tumor suppressor p53 is negatively regulated by MDM2 (Iwakuma and Lozano, 2003). Nutlin (for Nutley inhibitor) is able to bind to a hydrophobic pocket of the MDM2 protein, can impair the interaction of p53 and MDM2 and thus the ubiquitination and subsequent degradation of p53 by MDM2 (Kussie et al., 1996; Vassilev et al., 2004). The RING ligases RNF20 and RNF40 form an obligate heterodimer via multiple coiled-coil domains in their amino-terminal potions (Kim et al., 2009). Disruption of any of these motifs prevents dimerization and H2B monoubiquitination. The coiled-coil domain could therefore serve as a potential interaction surface for inhibitor design. Agents that impede the dimerization of RNF20 and RNF40 via their coiled-coil domains might function to disrupt the whole complex and could be used as therapeutic targets aimed at manipulating cancer cells for their selective elimination. 
Tumors of an earlier stage, which still exhibit significant levels of H2Bub1 may benefit from CDK9 inhibitors, since H2Bub1 can be decreased upon treatment (Minsky et al., 2008; Pirngruber et al., 2009a, 2009b). The usage of specific CDK9 inhibitors, like flavopiridol showed just poor therapeutic potential but strengthened the efficiency of DNA damaging agents (Hara et al., 2008; Mason et al., 2004; Motwani et al., 2001). A possible explanation why loss of H2Bub1 could be a benefit in this case is the connection to DNA damging, since loss of H2Bub1 could predict increased sensitivity of tumor cells to chemo- and radiotherapy by blocking H2Bub1-dependent DNA repair processes. H2Bub1 negative tumors might show higher sensitivity to irradiation than H2Bub1 positive tumors. In addition, based on the findings in ES cells, an increase in H2Bub1 is required for differentiation as already mentioned above. On the other hand tumors with low H2Bub1 levels should be controlled since it can lead to even greater decrease of H2Bub1 and thus increased malignant transformation. In addition, bromodomain-containing protein BRD4 which recruits CDK9 to transcribed genes (Jang et al., 2005; Yang et al., 2005b), could be another interesting target. Further, several specific small molecule inhibitors of CDK9 were already described as anticancer agents (Dai and Grant, 2004). Thus, different types of malignancies should be examined to shed more light into the role of H2Bub1 and its upstream regulatory pathway components.

Despite the fact that most studies indicate H2Bub1 and its ubiquitin ligases as tumor suppressors, a few data show a potential tumor promoting effect (Blank et al., 2012; Jaaskelainen et al., 2012; Liu et al., 2009). These effects could be explained by tumor-stage dependency. Particularly, H2Bub1 may play a role in suppression of metastasis and cancer stem cell phenotypes. A confirmation of this suggestion comes from the data of the present study where RNF20 and RNF40 knockdown decrease cell proliferation but increase cell migration. Further studies supported these findings and additionally showed an increased in in vivo tumor load (Shema et al., 2008). Again this explanation is in agreement with our data showing the loss of H2Bub1 during tumor progression in colon cancer samples and the observation of detectable H2Bub1 levels in early stage tumors and the absence in malignant and metastatic breast cancers (Prenzel et al., 2011). Consistently as already mentioned, 
USP22 correlates with poor prognosis and a stem cell gene expression signature (Glinsky et al., 2005).

Altogether, H2Bub1 can be used as an important marker of differentiated, nontransformed tissue and reduced H2Bub1 levels can possibly be used as a potential diagnostic tool or target for epigenetic-based therapies of cancer. The results presented here provide new insights into the role and regulation of $\mathrm{H} 2 \mathrm{~B}$ monoubiquitination and reveal a strong rationale for further pursuing subsequent genome- and transcriptome-wide analyses, in vivo mouse tumor model experiments and immunohistochemical studies with larger patient cohorts with follow-up data. Together these results will help establish the role of H2Bub1 in tumorigenesis, tumor progression and therapeutic responsiveness.

\subsection{Genome-wide distribution of H2Bub1}

As it was demonstrated in this study, treatment with stress-inducing factors led to a decrease in global H2Bub1 levels. Our ChIP-seq data confirmed the genome-wide increase in the gene transcribed region which is consistent with the increase of global H2Bub1 levels observed by Western blotting and former ChIP experiments. An accumulation of H2Bub1 on RNF20/RNF40 regulated genes would likely account for the observed increase of the H2Bub1 level. H2Bub1 was shown to be associated with the transcribed region of active genes (Minsky et al., 2008). A key issue that remains to be elucidated is whether H2Bub1 in the transcribed region has a direct function in transcription elongation. If H2Bub1 facilitates the elongation by RNAPII, a global defect in gene expression would be expected in cells impaired for H2B ubiquitination. However, in contrast to the distribution of H2Bub1 on most active genes, suppression of $\mathrm{H} 2 \mathrm{~B}$ ubiquitination appears to have a mild effect on gene expression as only a small subset of genes had an altered expression after depletion of the H2B ubiquitination enzymes in human cells. This suggests that instead of directly facilitating transcriptional elongation, H2Bub1 that is deposited along with elongating RNAPII could have functions independent of the transcription elongation itself. Since our data demonstrated that H2Bub1 levels are high across the body of some genes and quite strong at the 5' end of others genes this difference could 
potentially explain differential regulation of genes. The analysis of the genome-wide averages of H2Bub1 in control compared to ATXN7L3 depleted cells performed in this study demonstrated that average H2Bub1 might not correlate with gene expression but that the deubiquitination does correlate with gene activity.

A recent study revealed that deletion of CHD1, a chromatin remodeler required for maintaining high H2Bub1 levels resulted in a decrease in global H2Bub1 whereas both H3K4 and H3K79 trimethylation were not affected. Thus, CHD1 positively regulates gene expression through promoting nucleosome reassembly coupled with H2B monoubiquitination (Lee et al., 2012). Furthermore, H2Bub1 could be involved in transcription of micro-RNAs (miRNAs) or long non-coding RNAs (IncRNAs) which have shown to be recruited for differentiation (Crippa et al., 2012; Dong et al., 2012; van Leeuwen and Mikkers, 2010). To determine the effect of the loss of ATXN7L3 on overall gene expression and to correlate changes in RNA levels with changes in H2Bub1 enrichment additional RNA-seq analyses should be performed.

Our results obtained in MCF10A cells indicate that the SAGA DUBm has a much more widespread activity on H2Bub1 than previously anticipated. Indeed, the increase in H2Bub1 levels upon ATXN7L3 knockdown in the transcribed region of highly expressed genes seems to be widespread indicating that SAGA is likely recruited to all these genes and acts throughout the gene bodies. The role of SAGA has been extensively studied in yeast where it is essential for GAL1 transcription via binding to the promoter prior to recruitment of RNAPII and pre-initiation complex formation (Bhaumik and Green, 2001; Bryant and Ptashne, 2003; Larschan and Winston, 2001). Furthermore, SAGA binds to promoters of many different genes in several metazoan species (Lang et al., 2011; Nagy et al., 2009; Weake et al., 2011). In addition, it was shown that SAGA subunits, including ATXN7L3 localize to gene promoters linked to stress response, transcriptional regulation and replication (Lang et al., 2011; Nagy et al., 2009; Vermeulen et al., 2010). Since all these studies showed that SAGA is required to be recruited to gene promoters to activate transcription we anticipate that ATXN7L3 may also be localized near the 5' end of these genes. This is supported by our results which showed that the increase of H2Bub1 occurs primarily around the 5' end of genes. However, studies of the genome-wide recruitment of ATXN7L3 and comparisons to H2Bub1 occupancy at 
certain regions of the genes could shed important insight into the role of the SAGA DUBm complex in controlling dynamic H2B monoubiquitination.

Taken into account that dynamic changes of H2Bub1 levels upon stress induction do correlate to gene expression, it may be possible to utilize this mechanism to affect gene expression levels. Recently our group could show that proteasome inhibition caused a rapid loss of H2Bub1 and decreased chromatin dynamics (Prenzel et al., 2011). Based on the results represented here proteasome inhibition via MG132 or Bortezomib may specifically decrease H2Bub1 on the most highly transcribed genes.

All together our data reveal a tight regulation for the setting and the removal of H2Bub1 that appears to be extremely dynamic. Further analysis of our ChIP-seq data as well as ChIP-seq against ATXN7L3 to determine binding profiles investigating the genome-wide co-occupancy of H2Bub1 and ATXN7L3 might shed further light on this question. Furthermore RNA-seq analyses upon ATXN7L3 knockdown will help determine how dynamic $\mathrm{H} 2 \mathrm{~B}$ monoubiquitination and deubiquitination control gene expression.

Collectively, this study reveals important insight into the complex mechanism of the regulation of histone $\mathrm{H} 2 \mathrm{~B}$ monoubiquitination. Our data demonstrate the validity of H2Bub1 and its ubiquitin ligases RNF20 and RNF40 as potential tumor suppressors. We could further show a stress-induced loss of H2Bub1 and a rescue of the global H2Bub1 levels through knockdown of a deubiquitinating enzyme or subunits which interact with it. To shed some more light on the proposed mechanisms of H2Bub1 regulation, further careful and extensive study of this process is required for the development of solid knowledge of stress-induced $\mathrm{H} 2 \mathrm{~B}$ deubiquitination and the role of $\mathrm{H} 2 \mathrm{~B}$ monoubiquitination during tumorigenesis and metastasis. Filling these gaps will provide an advance in understanding the role of H2Bub1 as well as the potential of epigenetic-based therapies for cancer. 


\section{Reference List}

Ahmad, Y., Boisvert, F.-M., Gregor, P., Cobley, A., and Lamond, A.I. (2009). NOPdb: Nucleolar Proteome Database--2008 update. Nucleic Acids Res. 37, D181-184.

Allan, J., Cowling, G.J., Harborne, N., Cattini, P., Craigie, R., and Gould, H. (1981). Regulation of the higher-order structure of chromatin by histones $\mathrm{H} 1$ and $\mathrm{H} 5$. J. Cell Biol. 90, 279-288.

Al-Baker, E.A., Boyle, J., Harry, R., and Kill, I.R. (2004). A p53-independent pathway regulates nucleolar segregation and antigen translocation in response to DNA damage induced by UV irradiation. Exp. Cell Res. 292, 179-186.

Barski, A., Cuddapah, S., Cui, K., Roh, T.Y., Schones, D.E., Wang, Z., Wei, G., Chepelev, I., and Zhao, K. (2007). High-resolution profiling of histone methylations in the human genome. Cell 129, 823-837.

Batta, K., Zhang, Z., Yen, K., Goffman, D.B., and Pugh, B.F. (2011). Genome-wide function of H2B ubiquitylation in promoter and genic regions. Genes Dev 25, 22542265.

Belotserkovskaya, R., Oh, S., Bondarenko, V.A., Orphanides, G., Studitsky, V.M., and Reinberg, D. (2003). FACT facilitates transcription-dependent nucleosome alteration. Science 301, 1090-1093.

Benjamini, Y., and Hochberg, Y. (1995). Controlling the False Discovery Rate - A Practical and Powerful Approach to Multiple Testing. J. R. Stat. Soc. Ser. BMethodol. 57, 289-300.

Berger, S.L. (2007). The complex language of chromatin regulation during transcription. Nature 447, 407-412.

Bergink, S., Salomons, F.A., Hoogstraten, D., Groothuis, T.A., de, W.H., Wu, J., Yuan, L., Citterio, E., Houtsmuller, A.B., Neefjes, J., et al. (2006). DNA damage triggers nucleotide excision repair-dependent monoubiquitylation of histone $\mathrm{H} 2 \mathrm{~A}$. Genes Dev 20, 1343-1352.

Bernstein, B.E., Mikkelsen, T.S., Xie, X., Kamal, M., Huebert, D.J., Cuff, J., Fry, B., Meissner, A., Wernig, M., Plath, K., et al. (2006a). A bivalent chromatin structure marks key developmental genes in embryonic stem cells. Cell 125, 315-326.

Bernstein, B.E., Mikkelsen, T.S., Xie, X., Kamal, M., Huebert, D.J., Cuff, J., Fry, B., Meissner, A., Wernig, M., Plath, K., et al. (2006b). A bivalent chromatin structure marks key developmental genes in embryonic stem cells. Cell 125, 315-326.

Beviglia, L., Matsumoto, K., Lin, C.-S., Ziober, B.L., and Kramer, R.H. (1997). Expression of the C-Met/HGF receptor in human breast carcinoma: Correlation with tumor progression. Int. J. Cancer 74, 301-309. 
Bhaumik, S.R., and Green, M.R. (2001). SAGA is an essential in vivo target of the yeast acidic activator Gal4p. Genes Dev. 15, 1935-1945.

Bickmore, W.A., and van Steensel, B. (2013). Genome architecture: domain organization of interphase chromosomes. Cell 152, 1270-1284.

Blank, M., Tang, Y., Yamashita, M., Burkett, S.S., Cheng, S.Y., and Zhang, Y.E. (2012). A tumor suppressor function of Smurf2 associated with controlling chromatin landscape and genome stability through RNF20. Nat.Med. 18, 227-234.

Bodem, J., Dobreva, G., Hoffmann-Rohrer, U., Iben, S., Zentgraf, H., Delius, H., Vingron, M., and Grummt, I. (2000). TIF-IA, the factor mediating growth-dependent control of ribosomal RNA synthesis, is the mammalian homolog of yeast Rrn3p. EMBO Rep. 1, 171-175.

Boisvert, F.-M., and Lamond, A.I. (2010). p53-Dependent subcellular proteome localization following DNA damage. Proteomics 10, 4087-4097.

Boisvert, F.-M., van Koningsbruggen, S., Navascués, J., and Lamond, A.I. (2007). The multifunctional nucleolus. Nat. Rev. Mol. Cell Biol. 8, 574-585.

Boisvert, F.-M., Lam, Y.W., Lamont, D., and Lamond, A.I. (2010). A quantitative proteomics analysis of subcellular proteome localization and changes induced by DNA damage. Mol. Cell. Proteomics Mcp 9, 457-470.

Bolstad, B.M., Irizarry, R.A., Astrand, M., and Speed, T.P. (2003). A comparison of normalization methods for high density oligonucleotide array data based on variance and bias. Bioinformatics. 19, 185-193.

Bonnet, J., Romier, C., Tora, L., and Devys, D. (2008). Zinc-finger UBPs: regulators of deubiquitylation. Trends Biochem. Sci. 33, 369-375.

Boulon, S., Westman, B.J., Hutten, S., Boisvert, F.-M., and Lamond, A.I. (2010). The nucleolus under stress. Mol. Cell 40, 216-227.

Bourbousse, C., Ahmed, I., Roudier, F., Zabulon, G., Blondet, E., Balzergue, S., Colot, V., Bowler, C., and Barneche, F. (2012). Histone H2B Monoubiquitination Facilitates the Rapid Modulation of Gene Expression during Arabidopsis Photomorphogenesis. Plos Genet. 8.

Bracken, A.P., and Helin, K. (2009). Polycomb group proteins: navigators of lineage pathways led astray in cancer. Nat. Rev. Cancer 9, 773-784.

Briggs, S.D., Bryk, M., Strahl, B.D., Cheung, W.L., Davie, J.K., Dent, S.Y., Winston, F., and Allis, C.D. (2001). Histone H3 lysine 4 methylation is mediated by Set1 and required for cell growth and rDNA silencing in Saccharomyces cerevisiae. Genes Dev. 15, 3286-3295.

Briggs, S.D., Xiao, T., Sun, Z.W., Caldwell, J.A., Shabanowitz, J., Hunt, D.F., Allis, C.D., and Strahl, B.D. (2002). Gene silencing: trans-histone regulatory pathway in chromatin. Nature 418, 498. 
Brownell, J.E., Zhou, J., Ranalli, T., Kobayashi, R., Edmondson, D.G., Roth, S.Y., and Allis, C.D. (1996). Tetrahymena histone acetyltransferase A: a homolog to yeast Gcn5p linking histone acetylation to gene activation. Cell 84, 843-851.

Bryant, G.O., and Ptashne, M. (2003). Independent recruitment in vivo by Gal4 of two complexes required for transcription. Mol. Cell 11, 1301-1309.

Bungard, D., Fuerth, B.J., Zeng, P.-Y., Faubert, B., Maas, N.L., Viollet, B., Carling, D., Thompson, C.B., Jones, R.G., and Berger, S.L. (2010). Signaling kinase AMPK activates stress-promoted transcription via histone $\mathrm{H} 2 \mathrm{~B}$ phosphorylation. Science 329, 1201-1205.

Burger, K., Muhl, B., Harasim, T., Rohrmoser, M., Malamoussi, A., Orban, M., Kellner, M., Gruber-Eber, A., Kremmer, E., Holzel, M., et al. (2010). Chemotherapeutic Drugs Inhibit Ribosome Biogenesis at Various Levels. J. Biol. Chem. 285, 12416-12425.

Buszczak, M., Paterno, S., and Spradling, A.C. (2009). Drosophila stem cells share a common requirement for the histone H2B ubiquitin protease scrawny. Science 323, 248-251.

Camp, R.L., Rimm, E.B., and Rimm, D.L. (1999). Met expression is associated with poor outcome in patients with axillary lymph node negative breast carcinoma. Cancer 86, 2259-2265.

Cao, R., Tsukada, Y., and Zhang, Y. (2005). Role of Bmi-1 and Ring1A in H2A ubiquitylation and Hox gene silencing. Mol.Cell 20, 845-854.

Chaffer, C.L., and Weinberg, R.A. (2011). A Perspective on Cancer Cell Metastasis. Science 331, 1559-1564.

Chandrasekharan, M.B., Huang, F., and Sun, Z.W. (2009). Ubiquitination of histone H2B regulates chromatin dynamics by enhancing nucleosome stability. Proc.Natl.Acad.Sci.U.S.A 106, 16686-16691.

Chen, Y.-G., Wang, Q., Lin, S.-L., Chang, C.D., Chuang, J., Chung, J., and Ying, S.Y. (2006). Activin signaling and its role in regulation of cell proliferation, apoptosis, and carcinogenesis. Exp. Biol. Med. Maywood Nj 231, 534-544.

Chen, Y.-H., Hung, M.-C., and Li, L.-Y. (2012). EZH2: a pivotal regulator in controlling cell differentiation. Am. J. Transl. Res. 4, 364-375.

Chen, Y.R., Wang, X., Templeton, D., Davis, R.J., and Tan, T.H. (1996). The role of C-Jun $\mathrm{N}$-terminal kinase (JNK) in apoptosis induced by ultraviolet $\mathrm{C}$ and gamma radiation. Duration of JNK activation may determine cell death and proliferation. J. Biol. Chem. 271, 31929-31936.

Chernikova, S.B., Razorenova, O.V., Higgins, J.P., Sishc, B.J., Nicolau, M., Dorth, J.A., Chernikova, D.A., Kwok, S., Brooks, J.D., Bailey, S.M., et al. (2012). Deficiency in mammalian histone H2B ubiquitin ligase Bre1 (Rnf20/Rnf40) leads to replication stress and chromosomal instability. Cancer Res. 
Chi, P., Allis, C.D., and Wang, G.G. (2010). Covalent histone modifications: miswritten, misinterpreted, and miserased in human cancers. Nat. Rev. Cancer 10, 457-469.

Chin, L.S., Vavalle, J.P., and Li, L. (2002). Staring, a novel E3 ubiquitin-protein ligase that targets syntaxin 1 for degradation. Jbiol Chem 277, 35071-35079.

Chipumuro, E., and Henriksen, M.A. (2012). The ubiquitin hydrolase USP22 contributes to 3'-end processing of JAK-STAT-inducible genes. Faseb J 26, 842-854.

Christofk, H.R., Vander Heiden, M.G., Harris, M.H., Ramanathan, A., Gerszten, R.E., Wei, R., Fleming, M.D., Schreiber, S.L., and Cantley, L.C. (2008). The M2 splice isoform of pyruvate kinase is important for cancer metabolism and tumour growth. Nature 452, 230-233.

Cohen, A.A., Geva-Zatorsky, N., Eden, E., Frenkel-Morgenstern, M., Issaeva, I., Sigal, A., Milo, R., Cohen-Saidon, C., Liron, Y., Kam, Z., et al. (2008). Dynamic proteomics of individual cancer cells in response to a drug. Science 322, 1511-1516.

Colland, F. (2010). The therapeutic potential of deubiquitinating enzyme inhibitors. Biochem.Soc.Trans. 38, 137-143.

Crippa, S., Cassano, M., and Sampaolesi, M. (2012). Role of miRNAs in muscle stem cell biology: proliferation, differentiation and death. Curr. Pharm. Des. 18, 17181729.

Cui, Y., König, J., Buchholz, J.K., Spring, H., Leier, I., and Keppler, D. (1999). Drug resistance and ATP-dependent conjugate transport mediated by the apical multidrug resistance protein, MRP2, permanently expressed in human and canine cells. Mol. Pharmacol. 55, 929-937.

Dai, Y., and Grant, S. (2004). Small molecule inhibitors targeting cyclin-dependent kinases as anticancer agents. Curr. Oncol. Rep. 6, 123-130.

Daniel, J.A., Torok, M.S., Sun, Z.W., Schieltz, D., Allis, C.D., Yates, J.R., and Grant, P.A. (2004). Deubiquitination of histone H2B by a yeast acetyltransferase complex regulates transcription. J.Biol.Chem. 279, 1867-1871.

Davie, J.R., and Murphy, L.C. (1990). Level of ubiquitinated histone H2B in chromatin is coupled to ongoing transcription. Biochemistry (Mosc.) 29, 4752-4757.

Deng, L., Wang, C., Spencer, E., Yang, L., Braun, A., You, J., Slaughter, C., Pickart, C., and Chen, Z.J. (2000). Activation of the IkappaB kinase complex by TRAF6 requires a dimeric ubiquitin-conjugating enzyme complex and a unique polyubiquitin chain. Cell 103, 351-361.

Dérijard, B., Raingeaud, J., Barrett, T., Wu, I.H., Han, J., Ulevitch, R.J., and Davis, R.J. (1995). Independent human MAP-kinase signal transduction pathways defined by MEK and MKK isoforms. Science 267, 682-685. 
Ding, W.-X., Ni, H.-M., and Yin, X.-M. (2007). Absence of Bax switched MG132induced apoptosis to non-apoptotic cell death that could be suppressed by transcriptional or translational inhibition. Apoptosis Int. J. Program. Cell Death 12, 2233-2244.

Doil, C., Mailand, N., Bekker-Jensen, S., Menard, P., Larsen, D.H., Pepperkok, R., Ellenberg, J., Panier, S., Durocher, D., Bartek, J., et al. (2009). RNF168 binds and amplifies ubiquitin conjugates on damaged chromosomes to allow accumulation of repair proteins. Cell 136, 435-446.

Dong, S., Yang, B., Guo, H., and Kang, F. (2012). MicroRNAs regulate osteogenesis and chondrogenesis. Biochem. Biophys. Res. Commun. 418, 587-591.

Dover, J., Schneider, J., Tawiah-Boateng, M.A., Wood, A., Dean, K., Johnston, M., and Shilatifard, A. (2002). Methylation of histone $\mathrm{H3}$ by COMPASS requires ubiquitination of histone H2B by Rad6. Jbiol Chem 277, 28368-28371.

Drygin, D., Lin, A., Bliesath, J., Ho, C.B., O’Brien, S.E., Proffitt, C., Omori, M., Haddach, M., Schwaebe, M.K., Siddiqui-Jain, A., et al. (2011). Targeting RNA polymerase I with an oral small molecule CX-5461 inhibits ribosomal RNA synthesis and solid tumor growth. Cancer Res. 71, 1418-1430.

Egloff, S., and Murphy, S. (2008). Cracking the RNA polymerase II CTD code. Trends Genet 24, 280-288.

Emre, N.C., Ingvarsdottir, K., Wyce, A., Wood, A., Krogan, N.J., Henry, K.W., Li, K., Marmorstein, R., Greenblatt, J.F., Shilatifard, A., et al. (2005). Maintenance of low histone ubiquitylation by Ubp10 correlates with telomere-proximal Sir2 association and gene silencing. Mol.Cell 17, 585-594.

Eskeland, R., Leeb, M., Grimes, G.R., Kress, C., Boyle, S., Sproul, D., Gilbert, N., Fan, Y., Skoultchi, A.I., Wutz, A., et al. (2010). Ring1B compacts chromatin structure and represses gene expression independent of histone ubiquitination. Mol. Cell 38, $452-464$.

Farin, A., Suzuki, S.O., Weiker, M., Goldman, J.E., Bruce, J.N., and Canoll, P. (2006). Transplanted glioma cells migrate and proliferate on host brain vasculature: a dynamic analysis. Glia 53, 799-808.

Farrow, B., and Evers, B.M. (2002). Inflammation and the development of pancreatic cancer. Surg. Oncol. 10, 153-169.

Felsenfeld, G., and Groudine, M. (2003). Controlling the double helix. Nature 421, 448-453.

Fierz, B., Chatterjee, C., McGinty, R.K., Bar-Dagan, M., Raleigh, D.P., and Muir, T.W. (2011). Histone H2B ubiquitylation disrupts local and higher-order chromatin compaction. Nat.Chem.Biol 7, 113-119.

Fischle, W., Wang, Y., and Allis, C.D. (2003). Histone and chromatin cross-talk. Curropincell Biol 15, 172-183. 
Fisslthaler, B., and Fleming, I. (2009). Activation and signaling by the AMP-activated protein kinase in endothelial cells. Circ. Res. 105, 114-127.

Fleming, A.B., Kao, C.F., Hillyer, C., Pikaart, M., and Osley, M.A. (2008). H2B ubiquitylation plays a role in nucleosome dynamics during transcription elongation. Mol.Cell 31, 57-66.

Fraga, M.F., Ballestar, E., Villar-Garea, A., Boix-Chornet, M., Espada, J., Schotta, G., Bonaldi, T., Haydon, C., Ropero, S., Petrie, K., et al. (2005). Loss of acetylation at Lys 16 and trimethylation at Lys20 of histone $\mathrm{H} 4$ is a common hallmark of human cancer. Nat. Genet. 37, 391-400.

Francis, N.J., Kingston, R.E., and Woodcock, C.L. (2004). Chromatin compaction by a polycomb group protein complex. Science 306, 1574-1577.

Fuchs, G., Shema, E., Vesterman, R., Kotler, E., Wolchinsky, Z., Wilder, S., Golomb, L., Pribluda, A., Zhang, F., Haj-Yahya, M., et al. (2012). RNF20 and USP44 regulate stem cell differentiation by modulating H2B monoubiquitylation. Mol. Cell 46, 662673.

Fujinaga, K., Irwin, D., Huang, Y., Taube, R., Kurosu, T., and Peterlin, B.M. (2004). Dynamics of human immunodeficiency virus transcription: P-TEFb phosphorylates $\mathrm{RD}$ and dissociates negative effectors from the transactivation response element. Molcell Biol 24, 787-795.

Gao, Z., and Xu, C.W. (2011). Glucose metabolism induces mono-ubiquitination of histone H2B in mammalian cells. Biochem. Biophys. Res. Commun. 404, 428-433.

Gardner, R.G., Nelson, Z.W., and Gottschling, D.E. (2005). Ubp10/Dot4p regulates the persistence of ubiquitinated histone $\mathrm{H} 2 \mathrm{~B}$ : distinct roles in telomeric silencing and general chromatin. Molcell Biol 25, 6123-6139.

Ghoussoub, R.A.D., Dillon, D.A., D'Aquila, T., Rimm, E.B., Fearon, E.R., and Rimm, D.L. (1998). Expression of c-met is a strong independent prognostic factor in breast carcinoma. Cancer 82, 1513-1520.

Glickman, M.H., and Ciechanover, A. (2002). The ubiquitin-proteasome proteolytic pathway: destruction for the sake of construction. Physiol. Rev. 82, 373-428.

Glinsky, G.V. (2005). Death-from-cancer signatures and stem cell contribution to metastatic cancer. Cell Cycle 4, 1171-1175.

Glinsky, G.V., Berezovska, O., and Glinskii, A.B. (2005). Microarray analysis identifies a death-from-cancer signature predicting therapy failure in patients with multiple types of cancer. J.Clin.Invest 115, 1503-1521.

Goldknopf, I.L., and Busch, H. (1977). Isopeptide linkage between nonhistone and histone 2A polypeptides of chromosomal conjugate-protein A24. Proc. Natl. Acad. Sci. U. S. A. 74, 864-868. 
Gomes, N.P., Bjerke, G., Llorente, B., Szostek, S.A., Emerson, B.M., and Espinosa, J.M. (2006). Gene-specific requirement for P-TEFb activity and RNA polymerase II phosphorylation within the p53 transcriptional program. Genes Dev 20, 601-612.

Gonzalez, M.E., Li, X., Toy, K., DuPrie, M., Ventura, A.C., Banerjee, M., Ljungman, M., Merajver, S.D., and Kleer, C.G. (2009). Downregulation of EZH2 decreases growth of estrogen receptor-negative invasive breast carcinoma and requires BRCA1. Oncogene 28, 843-853.

Govoni, M., Farabegoli, F., Pession, A., and Novello, F. (1994). Inhibition of topoisomerase II activity and its effect on nucleolar structure and function. Exp. Cell Res. 211, 36-41.

Grant, P.A., Duggan, L., Côté, J., Roberts, S.M., Brownell, J.E., Candau, R., Ohba, R., Owen-Hughes, T., Allis, C.D., Winston, F., et al. (1997). Yeast Gen5 functions in two multisubunit complexes to acetylate nucleosomal histones: characterization of an Ada complex and the SAGA (Spt/Ada) complex. Genes Dev. 11, 1640-1650.

Grewal, S.I.S., and Elgin, S.C.R. (2007). Transcription and RNA interference in the formation of heterochromatin. Nature 447, 399-406.

Gurskiy, D., Orlova, A., Vorobyeva, N., Nabirochkina, E., Krasnov, A., Shidlovskii, Y., Georgieva, S., and Kopytova, D. (2012). The DUBm subunit Sgf11 is required for mRNA export and interacts with Cbp80 in Drosophila. Nucleic Acids Res. 40, 1068910700 .

Hahn, M.A., Dickson, K.A., Jackson, S., Clarkson, A., Gill, A.J., and Marsh, D.J. (2012). The tumor suppressor CDC73 interacts with the ring finger proteins RNF20 and RNF40 and is required for the maintenance of histone 2B monoubiquitination. Hum.Mol.Genet. 21, 559-568.

Hanahan, D., and Weinberg, R.A. (2011). Hallmarks of Cancer: The Next Generation. Cell 144, 646-674.

Hara, T., Omura-Minamisawa, M., Kang, Y., Cheng, C., and Inoue, T. (2008). Flavopiridol potentiates the cytotoxic effects of radiation in radioresistant tumor cells in which p53 is mutated or Bcl-2 is overexpressed. Intjradiatoncolbiol Phys 71, 14851495.

Hardie, D.G., Salt, I.P., Hawley, S.A., and Davies, S.P. (1999). AMP-activated protein kinase: an ultrasensitive system for monitoring cellular energy charge. Biochem. J. 338 ( Pt 3), 717-722.

Hawley, S.A., Boudeau, J., Reid, J.L., Mustard, K.J., Udd, L., Mäkelä, T.P., Alessi, D.R., and Hardie, D.G. (2003). Complexes between the LKB1 tumor suppressor, STRAD alpha/beta and MO25 alpha/beta are upstream kinases in the AMP-activated protein kinase cascade. J. Biol. 2, 28.

Heintzman, N.D., and Ren, B. (2009). Finding distal regulatory elements in the human genome. Curr. Opin. Genet. Dev. 19, 541-549. 
Helmlinger, D., Marguerat, S., Villén, J., Gygi, S.P., Bähler, J., and Winston, F. (2008). The S. pombe SAGA complex controls the switch from proliferation to sexual differentiation through the opposing roles of its subunits Gcn5 and Spt8. Genes Dev. 22, 3184-3195.

Henikoff, S. (2000). Heterochromatin function in complex genomes. Biochim. Biophys. Acta 1470, 01-8.

Henry, K.W., Wyce, A., Lo, W.S., Duggan, L.J., Emre, N.C., Kao, C.F., Pillus, L., Shilatifard, A., Osley, M.A., and Berger, S.L. (2003). Transcriptional activation via sequential histone H2B ubiquitylation and deubiquitylation, mediated by SAGAassociated Ubp8. Genes Dev 17, 2648-2663.

Heo, K., Kim, H., Choi, S.H., Choi, J., Kim, K., Gu, J., Lieber, M.R., Yang, A.S., and An, W. (2008). FACT-mediated exchange of histone variant H2AX regulated by phosphorylation of H2AX and ADP-ribosylation of Spt16. Mol.Cell 30, 86-97.

Hill, C.R., Jamieson, D., Thomas, H.D., Brown, C.D.A., Boddy, A.V., and Veal, G.J. (2013). Characterisation of the roles of $A B C B 1, A B C C 1, A B C C 2$ and $A B C G 2$ in the transport and pharmacokinetics of actinomycin $D$ in vitro and in vivo. Biochem. Pharmacol. 85, 29-37.

Hochstrasser, M. (1996). Protein degradation or regulation: Ub the judge. Cell 84, 813-815.

Hoege, C., Pfander, B., Moldovan, G.L., Pyrowolakis, G., and Jentsch, S. (2002). RAD6-dependent DNA repair is linked to modification of PCNA by ubiquitin and SUMO. Nature 419, 135-141.

Hofmann, R.M., and Pickart, C.M. (1999). Noncanonical MMS2-encoded ubiquitinconjugating enzyme functions in assembly of novel polyubiquitin chains for DNA repair. Cell 96, 645-653.

Hsu, P.P., and Sabatini, D.M. (2008). Cancer Cell Metabolism: Warburg and Beyond. Cell 134, 703-707.

Hwang, W.W., Venkatasubrahmanyam, S., lanculescu, A.G., Tong, A., Boone, C., and Madhani, H.D. (2003). A conserved RING finger protein required for histone H2B monoubiquitination and cell size control. Mol.Cell 11, 261-266.

Imhof, A. (2003). Histone modifications--marks for gene expression? Adv. Exp. Med. Biol. 544, 169-180.

Ingvarsdottir, K., Krogan, N.J., Emre, N.C.T., Wyce, A., Thompson, N.J., Emili, A., Hughes, T.R., Greenblatt, J.F., and Berger, S.L. (2005). H2B ubiquitin protease Ubp8 and Sgf11 constitute a discrete functional module within the Saccharomyces cerevisiae SAGA complex. Mol. Cell. Biol. 25, 1162-1172.

Iwakuma, T., and Lozano, G. (2003). MDM2, an introduction. Mol. Cancer Res. Mcr $1,993-1000$. 
Jaaskelainen, T., Makkonen, H., Visakorpi, T., Kim, J., Roeder, R.G., and Palvimo, J.J. (2012). Histone H2B ubiquitin ligases RNF20 and RNF40 in androgen signaling and prostate cancer cell growth. Molcell Endocrinol 350, 87-98.

Jang, M.K., Mochizuki, K., Zhou, M., Jeong, H.S., Brady, J.N., and Ozato, K. (2005). The bromodomain protein Brd4 is a positive regulatory component of P-TEFb and stimulates RNA polymerase II-dependent transcription. Mol.Cell 19, 523-534.

Jeffers, M., Fiscella, M., Webb, C.P., Anver, M., Koochekpour, S., and Woude, G.F.V. (1998). The mutationally activated Met receptor mediates motility and metastasis. Proc. Natl. Acad. Sci. 95, 14417-14422.

Jenuwein, T., and Allis, C.D. (2001). Translating the histone code. Science 293, 1074-1080.

Jeon, S.-M., Chandel, N.S., and Hay, N. (2012). AMPK regulates NADPH homeostasis to promote tumour cell survival during energy stress. Nature 485, 661665 .

Ji, X., Li, W., Song, J., Wei, L., and Liu, X.S. (2006). CEAS: cis-regulatory element annotation system. Nucleic Acids Res. 34, W551-554.

Johnsen, S.A. (2012a). The enigmatic role of H2Bub1 in cancer. Febs Lett 586, $1592-1601$.

Johnsen, S.A. (2012b). CDK9 and H2B Monoubiquitination: A Well-Choreographed Dance. PLoS.Genet. 8, e1002860.

Jung, I., Kim, S.K., Kim, M., Han, Y.M., Kim, Y.S., Kim, D., and Lee, D. (2012). H2B monoubiquitylation is a 5'-enriched active transcription mark and correlates with exon-intron structure in human cells. Genome Res 22, 1026-1035.

Kao, C.-F., Hillyer, C., Tsukuda, T., Henry, K., Berger, S., and Osley, M.A. (2004). Rad6 plays a role in transcriptional activation through ubiquitylation of histone H2B. Genes Dev. 18, 184-195.

Kari, V., Shchebet, A., Neumann, H., and Johnsen, S.A. (2011). The H2B ubiquitin ligase RNF40 cooperates with SUPT16H to induce dynamic changes in chromatin structure during DNA double-strand break repair. Cell Cycle 10, 3495-3504.

Karpiuk, O., Najafova, Z., Kramer, F., Hennion, M., Galonska, C., K"nig, A., Snaidero, N., Vogel, T., Shchebet, A., Begus-Nahrmann, Y., et al. (2012). The histone H2B monoubiquitination regulatory pathway is required for differentiation of multipotent stem cells. Mol.Cell 46, 705-713.

Kartalou, M., and Essigmann, J.M. (2001). Mechanisms of resistance to cisplatin. Mutat. Res. 478, 23-43.

Kato, S., Inoue, K., and Youn, M.-Y. (2010). Emergence of the osteo-epigenome in bone biology. Ibms Bonekey 7, 314-324. 
Keller, K.E., Tan, I.S., and Lee, Y.-S. (2012). SAICAR stimulates pyruvate kinase isoform $\mathrm{M} 2$ and promotes cancer cell survival in glucose-limited conditions. Science 338, 1069-1072.

Kharchenko, P.V., Alekseyenko, A.A., Schwartz, Y.B., Minoda, A., Riddle, N.C., Ernst, J., Sabo, P.J., Larschan, E., Gorchakov, A.A., Gu, T., et al. (2011). Comprehensive analysis of the chromatin landscape in Drosophila melanogaster. Nature 471, 480-485.

Kim, J., Hake, S.B., and Roeder, R.G. (2005). The human homolog of yeast BRE1 functions as a transcriptional coactivator through direct activator interactions. Mol.Cell 20, 759-770.

Kim, J., Guermah, M., McGinty, R.K., Lee, J.S., Tang, Z., Milne, T.A., Shilatifard, A., Muir, T.W., and Roeder, R.G. (2009). RAD6-Mediated transcription-coupled H2B ubiquitylation directly stimulates H3K4 methylation in human cells. Cell 137, 459471.

Kinyamu, H.K., Chen, J., and Archer, T.K. (2005). Linking the ubiquitin-proteasome pathway to chromatin remodeling/modification by nuclear receptors. J. Mol. Endocrinol. 34, 281-297.

Kireeva, M.L., Walter, W., Tchernajenko, V., Bondarenko, V., Kashlev, M., and Studitsky, V.M. (2002). Nucleosome remodeling induced by RNA polymerase II: loss of the $\mathrm{H} 2 \mathrm{~A} / \mathrm{H} 2 \mathrm{~B}$ dimer during transcription. Mol. Cell 9, 541-552.

Kirisako, T., Kamei, K., Murata, S., Kato, M., Fukumoto, H., Kanie, M., Sano, S., Tokunaga, F., Tanaka, K., and Iwai, K. (2006). A ubiquitin ligase complex assembles linear polyubiquitin chains. Embo J. 25, 4877-4887.

Van der Knaap, J.A., Kumar, B.R., Moshkin, Y.M., Langenberg, K., Krijgsveld, J., Heck, A.J., Karch, F., and Verrijzer, C.P. (2005). GMP synthetase stimulates histone H2B deubiquitylation by the epigenetic silencer USP7. Mol.Cell 17, 695-707.

Van der Knaap, J.A., Kozhevnikova, E., Langenberg, K., Moshkin, Y.M., and Verrijzer, C.P. (2010). Biosynthetic enzyme GMP synthetase cooperates with ubiquitin-specific protease 7 in transcriptional regulation of ecdysteroid target genes. Mol. Cell. Biol. 30, 736-744.

Kohler, A., Pascual-Garcia, P., Llopis, A., Zapater, M., Posas, F., Hurt, E., and Rodriguez-Navarro, S. (2006a). The mRNA Export Factor Sus1 Is Involved in Spt/Ada/Gcn5 Acetyltransferase-mediated H2B Deubiquitinylation through Its Interaction with Ubp8 and Sgf11. Mol. Biol. Cell 17, 4228-4236.

Kohler, A., Pascual-Garcia, P., Llopis, A., Zapater, M., Posas, F., Hurt, E., and Rodriguez-Navarro, S. (2006b). The mRNA export factor Sus1 is involved in Spt/Ada/Gcn5 acetyltransferase-mediated H2B deubiquitinylation through its interaction with Ubp8 and Sgf11. Molbiol Cell 17, 4228-4236. 
Kohler, A., Zimmerman, E., Schneider, M., Hurt, E., and Zheng, N. (2010). Structural basis for assembly and activation of the heterotetrameric SAGA histone $\mathrm{H} 2 \mathrm{~B}$ deubiquitinase module. Cell 141, 606-617.

Koken, M.H., Reynolds, P., Jaspers-Dekker, I., Prakash, L., Prakash, S., Bootsma, D., and Hoeijmakers, J.H. (1991). Structural and functional conservation of two human homologs of the yeast DNA repair gene RAD6. Proc. Natl. Acad. Sci. U. S. A. 88, 8865-8869.

Kolas, N.K., Chapman, J.R., Nakada, S., Ylanko, J., Chahwan, R., Sweeney, F.D., Panier, S., Mendez, M., Wildenhain, J., Thomson, T.M., et al. (2007). Orchestration of the DNA-damage response by the RNF8 ubiquitin ligase. Science 318, 16371640.

Kool, M., de Haas, M., Scheffer, G.L., Scheper, R.J., van Eijk, M.J., Juijn, J.A., Baas, F., and Borst, P. (1997). Analysis of expression of cMOAT (MRP2), MRP3, MRP4, and MRP5, homologues of the multidrug resistance-associated protein gene (MRP1), in human cancer cell lines. Cancer Res. 57, 3537-3547.

Koppenol, W.H., Bounds, P.L., and Dang, C.V. (2011). Otto Warburg's contributions to current concepts of cancer metabolism. Nat. Rev. Cancer 11, 325-337.

Kopytova, D.V., Krasnov, A.N., Orlova, A.V., Gurskiy, D.Y., Nabirochkina, E.N., Georgieva, S.G., and Shidlovskii, Y.V. (2010a). ENY2: couple, triple...more? Cell Cycle Georget. Tex 9, 479-481.

Kopytova, D.V., Orlova, A.V., Krasnov, A.N., Gurskiy, D.Y., Nikolenko, J.V., Nabirochkina, E.N., Shidlovskii, Y.V., and Georgieva, S.G. (2010b). Multifunctional factor ENY2 is associated with the THO complex and promotes its recruitment onto nascent mRNA. Genes Dev. 24, 86-96.

Kornberg, R.D. (1974). Chromatin structure: a repeating unit of histones and DNA. Science 184, 868-871.

Krogan, N.J., Dover, J., Khorrami, S., Greenblatt, J.F., Schneider, J., Johnston, M., and Shilatifard, A. (2002). COMPASS, a histone H3 (Lysine 4) methyltransferase required for telomeric silencing of gene expression. Jbiol Chem 277, 10753-10755.

Krogan, N.J., Dover, J., Wood, A., Schneider, J., Heidt, J., Boateng, M.A., Dean, K., Ryan, O.W., Golshani, A., Johnston, M., et al. (2003). The Paf1 complex is required for histone $\mathrm{H} 3$ methylation by COMPASS and Dot1p: linking transcriptional elongation to histone methylation. Mol.Cell 11, 721-729.

Kruse, J.-P., and Gu, W. (2009). Modes of p53 regulation. Cell 137, 609-622.

Ku, M., Koche, R.P., Rheinbay, E., Mendenhall, E.M., Endoh, M., Mikkelsen, T.S., Presser, A., Nusbaum, C., Xie, X., Chi, A.S., et al. (2008). Genomewide Analysis of PRC1 and PRC2 Occupancy Identifies Two Classes of Bivalent Domains. Plos Genet 4, e1000242. 
Kurshakova, M.M., Krasnov, A.N., Kopytova, D.V., Shidlovskii, Y.V., Nikolenko, J.V., Nabirochkina, E.N., Spehner, D., Schultz, P., Tora, L., and Georgieva, S.G. (2007). SAGA and a novel Drosophila export complex anchor efficient transcription and mRNA export to NPC. Embo J. 26, 4956-4965.

Kussie, P.H., Gorina, S., Marechal, V., Elenbaas, B., Moreau, J., Levine, A.J., and Pavletich, N.P. (1996). Structure of the MDM2 oncoprotein bound to the p53 tumor suppressor transactivation domain. Science 274, 948-953.

Laemmli, U.K. (1970). Cleavage of structural proteins during the assembly of the head of bacteriophage T4. Nature 227, 680-685.

Lahad, J.P., Mills, G.B., and Coombes, K.R. (2005). Stem cell-ness: a "magic marker" for cancer. J. Clin. Invest. 115, 1463-1467.

Lang, G., Bonnet, J., Umlauf, D., Karmodiya, K., Koffler, J., Stierle, M., Devys, D., and Tora, L. (2011). The tightly controlled deubiquitination activity of the human SAGA complex differentially modifies distinct gene regulatory elements. Molcell Biol $31,3734-3744$.

Laribee, R.N., Fuchs, S.M., and Strahl, B.D. (2007). H2B ubiquitylation in transcriptional control: a FACT-finding mission. Genes Dev 21, 737-743.

Larschan, E., and Winston, F. (2001). The S. cerevisiae SAGA complex functions in vivo as a coactivator for transcriptional activation by Gal4. Genes Dev. 15, 19461956.

Lee, J.T., and Gu, W. (2010). The multiple levels of regulation by p53 ubiquitination. Cell Death Differ. 17, 86-92.

Lee, J.S., Shukla, A., Schneider, J., Swanson, S.K., Washburn, M.P., Florens, L., Bhaumik, S.R., and Shilatifard, A. (2007). Histone crosstalk between H2B monoubiquitination and $\mathrm{H} 3$ methylation mediated by COMPASS. Cell 131, 10841096.

Lee, J.S., Garrett, A.S., Yen, K., Takahashi, Y.H., Hu, D., Jackson, J., Seidel, C., Pugh, B.F., and Shilatifard, A. (2012). Codependency of H2B monoubiquitination and nucleosome reassembly on Chd1. Genes Dev 26, 914-919.

Lee, K.K., Florens, L., Swanson, S.K., Washburn, M.P., and Workman, J.L. (2005). The Deubiquitylation Activity of Ubp8 Is Dependent upon Sgf11 and Its Association with the SAGA Complex. Mol. Cell. Biol. 25, 1173-1182.

Van Leeuwen, S., and Mikkers, H. (2010). Long non-coding RNAs: Guardians of development. Differ. Res. Biol. Divers. 80, 175-183.

Van Leeuwen, F., Gafken, P.R., and Gottschling, D.E. (2002). Dot1p modulates silencing in yeast by methylation of the nucleosome core. Cell 109, 745-756.

Lempiäinen, H., and Shore, D. (2009). Growth control and ribosome biogenesis. Curr. Opin. Cell Biol. 21, 855-863. 
Li, B., Howe, L., Anderson, S., Yates, J.R., and Workman, J.L. (2003). The Set2 histone methyltransferase functions through the phosphorylated carboxyl-terminal domain of RNA polymerase II. Jbiol Chem 278, 8897-8903.

Liang, T.J., Reid, A.E., Xavier, R., Cardiff, R.D., and Wang, T.C. (1996). Transgenic expression of tpr-met oncogene leads to development of mammary hyperplasia and tumors. J. Clin. Invest. 97, 2872-2877.

Lin, X., Skolnik, S., Chen, X., and Wang, J. (2011). Attenuation of intestinal absorption by major efflux transporters: quantitative tools and strategies using a Caco-2 model. Drug Metab. Dispos. Biol. Fate Chem. 39, 265-274.

Lis, E.T., and Romesberg, F.E. (2006). Role of Doa1 in the Saccharomyces cerevisiae DNA Damage Response. Mol. Cell. Biol. 26, 4122-4133.

Liu, F., Benashski, S.E., Persky, R., Xu, Y., Li, J., and McCullough, L.D. (2012). Agerelated changes in AMP-activated protein kinase after stroke. Age Dordr. Neth. 34, 157-168.

Liu, Y.L., Yang, Y.M., Xu, H., and Dong, X.S. (2010). Increased expression of ubiquitin-specific protease 22 can promote cancer progression and predict therapy failure in human colorectal cancer. J.Gastroenterol.Hepatol. 25, 1800-1805.

Liu, Y.L., Yang, Y.M., Xu, H., and Dong, X.S. (2011). Aberrant expression of USP22 is associated with liver metastasis and poor prognosis of colorectal cancer. J.Surg.Oncol. 103, 283-289.

Liu, Z., Ahn, J.-Y., Liu, X., and Ye, K. (2006). Ebp1 isoforms distinctively regulate cell survival and differentiation. Proc. Natl. Acad. Sci. U. S. A. 103, 10917-10922.

Liu, Z., Oh, S.M., Okada, M., Liu, X., Cheng, D., Peng, J., Brat, D.J., Sun, S.Y., Zhou, W., Gu, W., et al. (2009). Human BRE1 is an E3 ubiquitin ligase for Ebp1 tumor suppressor. Molbiol Cell 20, 757-768.

Luger, K., Mäder, A.W., Richmond, R.K., Sargent, D.F., and Richmond, T.J. (1997). Crystal structure of the nucleosome core particle at 2.8 A resolution. Nature 389, 251-260.

Mailand, N., Bekker-Jensen, S., Faustrup, H., Melander, F., Bartek, J., Lukas, C., and Lukas, J. (2007). RNF8 ubiquitylates histones at DNA double-strand breaks and promotes assembly of repair proteins. Cell 131, 887-900.

Margueron, R., and Reinberg, D. (2011). The Polycomb complex PRC2 and its mark in life. Nature 469, 343-349.

Marsden, M.P., and Laemmli, U.K. (1979). Metaphase chromosome structure: evidence for a radial loop model. Cell 17, 849-858.

Marteijn, J.A., Bekker-Jensen, S., Mailand, N., Lans, H., Schwertman, P., Gourdin, A.M., Dantuma, N.P., Lukas, J., and Vermeulen, W. (2009). Nucleotide excision 
repair-induced $\mathrm{H} 2 \mathrm{~A}$ ubiquitination is dependent on MDC1 and RNF8 and reveals a universal DNA damage response. Jcell Biol 186, 835-847.

Martindale, J.L., and Holbrook, N.J. (2002). Cellular response to oxidative stress: signaling for suicide and survival. J. Cell. Physiol. 192, 1-15.

Mason, K.A., Hunter, N.R., Raju, U., Ariga, H., Husain, A., Valdecanas, D., Neal, R., Ang, K.K., and Milas, L. (2004). Flavopiridol increases therapeutic ratio of radiotherapy by preferentially enhancing tumor radioresponse. Intjradiatoncolbiol Phys 59, 1181-1189.

Massagué, J. (1998). TGF-beta signal transduction. Annu. Rev. Biochem. 67, 753791.

Matsuoka, S., Ballif, B.A., Smogorzewska, A., McDonald, E.R., Hurov, K.E., Luo, J., Bakalarski, C.E., Zhao, Z., Solimini, N., Lerenthal, Y., et al. (2007). ATM and ATR substrate analysis reveals extensive protein networks responsive to DNA damage. Science 316, 1160-1166.

Mayer, C., and Grummt, I. (2005). Cellular stress and nucleolar function. Cell Cycle Georget. Tex 4, 1036-1038.

Mayer, C., and Grummt, I. (2006). Ribosome biogenesis and cell growth: mTOR coordinates transcription by all three classes of nuclear RNA polymerases. Oncogene 25, 6384-6391.

Mayer, C., Bierhoff, H., and Grummt, I. (2005). The nucleolus as a stress sensor: JNK2 inactivates the transcription factor TIF-IA and down-regulates rRNA synthesis. Genes Dev. 19, 933-941.

McCullough, S.D., Xu, X., Dent, S.Y.R., Bekiranov, S., Roeder, R.G., and Grant, P.A. (2012). Reelin is a target of polyglutamine expanded ataxin-7 in human spinocerebellar ataxia type 7 (SCA7) astrocytes. Proc. Natl. Acad. Sci. 109, 21319 21324.

Mikkelsen, T.S., Ku, M., Jaffe, D.B., Issac, B., Lieberman, E., Giannoukos, G., Alvarez, P., Brockman, W., Kim, T.-K., Koche, R.P., et al. (2007). Genome-wide maps of chromatin state in pluripotent and lineage-committed cells. Nature 448, 553560 .

Miller, C., Schwalb, B., Maier, K., Schulz, D., Dümcke, S., Zacher, B., Mayer, A., Sydow, J., Marcinowski, L., Dölken, L., et al. (2011). Dynamic transcriptome analysis measures rates of mRNA synthesis and decay in yeast. Mol. Syst. Biol. 7, 458.

Miller, T., Krogan, N.J., Dover, J., Erdjument-Bromage, H., Tempst, P., Johnston, M., Greenblatt, J.F., and Shilatifard, A. (2001). COMPASS: a complex of proteins associated with a trithorax-related SET domain protein. Proc. Natl. Acad. Sci. U. S. A. 98, 12902-12907. 
Minsky, N., Shema, E., Field, Y., Schuster, M., Segal, E., and Oren, M. (2008). Monoubiquitinated $\mathrm{H} 2 \mathrm{~B}$ is associated with the transcribed region of highly expressed genes in human cells. Natcell Biol 10, 483-488.

Monroe, D.G., Getz, B.J., Johnsen, S.A., Riggs, B.L., Khosla, S., and Spelsberg, T.C. (2003). Estrogen receptor isoform-specific regulation of endogenous gene expression in human osteoblastic cell lines expressing either ERalpha or ERbeta. Jcell Biochem 90, 315-326.

Moorefield, B., Greene, E.A., and Reeder, R.H. (2000). RNA polymerase I transcription factor Rrn3 is functionally conserved between yeast and human. Proc. Natl. Acad. Sci. U. S. A. 97, 4724-4729.

Motwani, M., Jung, C., Sirotnak, F.M., She, Y., Shah, M.A., Gonen, M., and Schwartz, G.K. (2001). Augmentation of apoptosis and tumor regression by flavopiridol in the presence of CPT-11 in Hct116 colon cancer monolayers and xenografts. Clincancer Res 7, 4209-4219.

Moyal, L., Lerenthal, Y., Gana-Weisz, M., Mass, G., So, S., Wang, S.Y., Eppink, B., Chung, Y.M., Shalev, G., Shema, E., et al. (2011). Requirement of ATM-dependent monoubiquitylation of histone $\mathrm{H} 2 \mathrm{~B}$ for timely repair of DNA double-strand breaks. Mol.Cell 41, 529-542.

Mu, J.J., Wang, Y., Luo, H., Leng, M., Zhang, J., Yang, T., Besusso, D., Jung, S.Y., and Qin, J. (2007). A proteomic analysis of ataxia telangiectasia-mutated (ATM)/ATM-Rad3-related (ATR) substrates identifies the ubiquitin-proteasome system as a regulator for DNA damage checkpoints. Jbiol Chem 282, 17330-17334.

Musi, N., Hirshman, M.F., Nygren, J., Svanfeldt, M., Bavenholm, P., Rooyackers, O., Zhou, G., Williamson, J.M., Ljunqvist, O., Efendic, S., et al. (2002). Metformin increases AMP-activated protein kinase activity in skeletal muscle of subjects with type 2 diabetes. Diabetes 51, 2074-2081.

Mutiu, A.I., Hoke, S.M.T., Genereaux, J., Liang, G., and Brandl, C.J. (2007). The role of histone ubiquitylation and deubiquitylation in gene expression as determined by the analysis of an HTB1(K123R) Saccharomyces cerevisiae strain. Mol. Genet. Genomics Mgg 277, 491-506.

Nagy, P.L., Griesenbeck, J., Kornberg, R.D., and Cleary, M.L. (2002). A trithoraxgroup complex purified from Saccharomyces cerevisiae is required for methylation of histone H3. Proc. Natl. Acad. Sci. U. S. A. 99, 90-94.

Nagy, Z., Riss, A., Romier, C., le Guezennec, X., Dongre, A.R., Orpinell, M., Han, J., Stunnenberg, H., and Tora, L. (2009). The human SPT20-containing SAGA complex plays a direct role in the regulation of endoplasmic reticulum stress-induced genes. Mol. Cell. Biol. 29, 1649-1660.

Nakamura, K., Kato, A., Kobayashi, J., Yanagihara, H., Sakamoto, S., Oliveira, D.V., Shimada, M., Tauchi, H., Suzuki, H., Tashiro, S., et al. (2011). Regulation of 
homologous recombination by RNF20-dependent H2B ubiquitination. Mol.Cell 41, 515-528.

Naldini, L., Vigna, E., Narsimhan, R.P., Gaudino, G., Zarnegar, R., Michalopoulos, G.K., and Comoglio, P.M. (1991). Hepatocyte growth factor (HGF) stimulates the tyrosine kinase activity of the receptor encoded by the proto-oncogene c-MET. Oncogene 6, 501-504.

Narasaki, F., Oka, M., Nakano, R., Ikeda, K., Fukuda, M., Nakamura, T., Soda, H., Nakagawa, M., Kuwano, M., and Kohno, S. (1997). Human canalicular multispecific organic anion transporter (cMOAT) is expressed in human lung, gastric, and colorectal cancer cells. Biochem. Biophys. Res. Commun. 240, 606-611.

Neri, F., Zippo, A., Krepelova, A., Cherubini, A., Rocchigiani, M., and Oliviero, S. (2012). Myc Regulates the Transcription of the PRC2 Gene To Control the Expression of Developmental Genes in Embryonic Stem Cells. Mol. Cell. Biol. 32, 840-851.

Ng, H.H., Feng, Q., Wang, H., Erdjument-Bromage, H., Tempst, P., Zhang, Y., and Struhl, K. (2002a). Lysine methylation within the globular domain of histone H3 by Dot1 is important for telomeric silencing and Sir protein association. Genes Dev. 16, 1518-1527.

Ng, H.H., Xu, R.M., Zhang, Y., and Struhl, K. (2002b). Ubiquitination of histone H2B by Rad6 is required for efficient Dot1-mediated methylation of histone H3 lysine 79. J.Biol.Chem. 277, 34655-34657.

Ng, H.H., Ciccone, D.N., Morshead, K.B., Oettinger, M.A., and Struhl, K. (2003). Lysine-79 of histone $\mathrm{H} 3$ is hypomethylated at silenced loci in yeast and mammalian cells: a potential mechanism for position-effect variegation. Proc. Natl. Acad. Sci. U. S. A. $100,1820-1825$.

Nicassio, F., Corrado, N., Vissers, J.H., Areces, L.B., Bergink, S., Marteijn, J.A., Geverts, B., Houtsmuller, A.B., Vermeulen, W., Di Fiore, P.P., et al. (2007). Human USP3 Is a Chromatin Modifier Required for S Phase Progression and Genome Stability. Curr.Biol 17, 1972-1977.

O'Neill, L.A. (2000). The interleukin-1 receptor/Toll-like receptor superfamily: signal transduction during inflammation and host defense. Sci. Stke Signal Transduct. Knowl. Environ. 2000, re1.

Okada, Y., Feng, Q., Lin, Y., Jiang, Q., Li, Y., Coffield, V.M., Su, L., Xu, G., and Zhang, Y. (2005). hDOT1L links histone methylation to leukemogenesis. Cell 121, 167-178.

Osley, M.A. (2004). H2B ubiquitylation: the end is in sight. Biochim. Biophys. Acta 1677, 74-78.

Osley, M.A. (2006). Regulation of histone H2A and H2B ubiquitylation. Brief.Funct.Genomic.Proteomic. 5, 179-189. 
Park, P.J. (2009). ChIP-seq: advantages and challenges of a maturing technology. Nat. Rev. Genet. 10, 669-680.

Park, G., Gong, Z., Chen, J., and Kim, J.E. (2010). Characterization of the DOT1L network: implications of diverse roles for DOT1L. Protein J 29, 213-223.

Pascual-García, P., and Rodríguez-Navarro, S. (2009). A tale of coupling, Sus1 function in transcription and mRNA export. Rna Biol. 6, 141-144.

Pavri, R., Zhu, B., Li, G., Trojer, P., Mandal, S., Shilatifard, A., and Reinberg, D. (2006). Histone H2B monoubiquitination functions cooperatively with FACT to regulate elongation by RNA polymerase II. Cell 125, 703-717.

Pederson, T., and Tsai, R.Y.L. (2009). In search of nonribosomal nucleolar protein function and regulation. J. Cell Biol. 184, 771-776.

Pirngruber, J., Shchebet, A., Schreiber, L., Shema, E., Minsky, N., Chapman, R.D., Eick, D., Aylon, Y., Oren, M., and Johnsen, S.A. (2009a). CDK9 directs H2B monoubiquitination and controls replication-dependent histone mRNA 3' end processing. Embo Rep 10, 894-900.

Pirngruber, J., Shchebet, A., and Johnsen, S.A. (2009b). Insights into the function of the human $\mathrm{P}-\mathrm{TEFb}$ component CDK9 in the regulation of chromatin modifications and co-transcriptional mRNA processing. Cell Cycle 8, 3636-3642.

Prenzel, T., Begus-Nahrmann, Y., Kramer, F., Hennion, M., Hsu, C., Gorsler, T., Hintermair, C., Eick, D., Kremmer, E., Simons, M., et al. (2011). Estrogen-Dependent Gene Transcription in Human Breast Cancer Cells Relies upon ProteasomeDependent Monoubiquitination of Histone H2B. Cancer Res 71, 5739-5753.

Ramalho-Santos, M., Yoon, S., Matsuzaki, Y., Mulligan, R.C., and Melton, D.A. (2002). "Stemness": transcriptional profiling of embryonic and adult stem cells. Science 298, 597-600.

Rando, O.J., and Winston, F. (2012). Chromatin and transcription in yeast. Genetics 190, 351-387.

Rhodes, D.R., Yu, J., Shanker, K., Deshpande, N., Varambally, R., Ghosh, D., Barrette, T., Pandey, A., and Chinnaiyan, A.M. (2004). ONCOMINE: a cancer microarray database and integrated data-mining platform. Neoplasia New York $\mathrm{N} 6$, $1-6$.

Richards, E.J., and Elgin, S.C.R. (2002). Epigenetic codes for heterochromatin formation and silencing: rounding up the usual suspects. Cell 108, 489-500.

Richly, H., Lange, M., Simboeck, E., and Di Croce, L. (2010). Setting and resetting of epigenetic marks in malignant transformation and development. Bioessays News Rev. Mol. Cell. Dev. Biol. 32, 669-679.

Risbridger, G.P., Schmitt, J.F., and Robertson, D.M. (2001). Activins and Inhibins in Endocrine and Other Tumors. Endocr. Rev. 22, 836-858. 
Robertson, G., Hirst, M., Bainbridge, M., Bilenky, M., Zhao, Y., Zeng, T., Euskirchen, G., Bernier, B., Varhol, R., Delaney, A., et al. (2007). Genome-wide profiles of STAT1 DNA association using chromatin immunoprecipitation and massively parallel sequencing. Nat. Methods 4, 651-657.

Robzyk, K., Recht, J., and Osley, M.A. (2000). Rad6-dependent ubiquitination of histone H2B in yeast. Science 287, 501-504.

Rodriguez, R.M., Huidobro, C., Urdinguio, R.G., Mangas, C., Soldevilla, B., Dominguez, G., Bonilla, F., Fernandez, A.F., and Fraga, M.F. (2012). Aberrant epigenetic regulation of bromodomain BRD4 in human colon cancer. J.Mol.Med.(Berl) 90, 587-595.

Rodriguez-Navarro, S. (2009). Insights into SAGA function during gene expression. Embo Rep 10, 843-850.

Roguev, A., Schaft, D., Shevchenko, A., Pijnappel, W.W., Wilm, M., Aasland, R., and Stewart, A.F. (2001). The Saccharomyces cerevisiae Set1 complex includes an Ash2 homologue and methylates histone 3 lysine 4. Embo J. 20, 7137-7148.

Roudier, F., Ahmed, I., Bérard, C., Sarazin, A., Mary-Huard, T., Cortijo, S., Bouyer, D., Caillieux, E., Duvernois-Berthet, E., Al-Shikhley, L., et al. (2011). Integrative epigenomic mapping defines four main chromatin states in Arabidopsis. Embo J. 30, 1928-1938.

Roy, S., Ernst, J., Kharchenko, P.V., Kheradpour, P., Negre, N., Eaton, M.L., Landolin, J.M., Bristow, C.A., Ma, L., Lin, M.F., et al. (2010). Identification of functional elements and regulatory circuits by Drosophila modENCODE. Science 330, 1787-1797.

Ben-Saadon, R., Zaaroor, D., Ziv, T., and Ciechanover, A. (2006). The polycomb protein Ring1B generates self atypical mixed ubiquitin chains required for its in vitro histone H2A ligase activity. Mol. Cell 24, 701-711.

Sandusky, G.E., Mintze, K.S., Pratt, S.E., and Dantzig, A.H. (2002). Expression of multidrug resistance-associated protein 2 (MRP2) in normal human tissues and carcinomas using tissue microarrays. Histopathology 41, 65-74.

Sans $\phi$, M., Lee, K.., Viladevall, L., Jacques, P.M., Pag,, V., Nagy, S., Racine, A., St Amour, C.V., Zhang, C., Shokat, K.M., et al. (2012). A positive feedback loop links opposing functions of $\mathrm{P}-\mathrm{TEFb} / \mathrm{Cck9}$ and histone $\mathrm{H} 2 \mathrm{~B}$ ubiquitylation to regulate transcript elongation in fission yeast. PLoS.Genet.

San-Segundo, P.A., and Roeder, G.S. (2000). Role for the Silencing Protein Dot1 in Meiotic Checkpoint Control. Mol. Biol. Cell 11, 3601-3615.

Schulz, E., Dopheide, J., Schuhmacher, S., Thomas, S.R., Chen, K., Daiber, A., Wenzel, P., Münzel, T., and Keaney, J.F., Jr (2008). Suppression of the JNK pathway by induction of a metabolic stress response prevents vascular injury and dysfunction. Circulation 118, 1347-1357. 
Schwabish, M.A., and Struhl, K. (2004). Evidence for eviction and rapid deposition of histones upon transcriptional elongation by RNA polymerase II. Mol. Cell. Biol. 24, 10111-10117.

Schwartz, Y.B., and Pirrotta, V. (2008). Polycomb complexes and epigenetic states. Curr. Opin. Cell Biol. 20, 266-273.

Shackelford, D.B., and Shaw, R.J. (2009). The LKB1-AMPK pathway: metabolism and growth control in tumour suppression. Nat. Rev. Cancer 9, 563-575.

Shav-Tal, Y., Blechman, J., Darzacq, X., Montagna, C., Dye, B.T., Patton, J.G., Singer, R.H., and Zipori, D. (2005). Dynamic sorting of nuclear components into distinct nucleolar caps during transcriptional inhibition. Mol. Biol. Cell 16, 2395-2413.

Shaw, R.J., Kosmatka, M., Bardeesy, N., Hurley, R.L., Witters, L.A., DePinho, R.A., and Cantley, L.C. (2004). The tumor suppressor LKB1 kinase directly activates AMPactivated kinase and regulates apoptosis in response to energy stress. Proc. Natl. Acad. Sci. U. S. A. 101, 3329-3335.

Shchebet, A. (2011). Investigations into the regulation of histone $\mathrm{H} 2 \mathrm{~B}$ monoubiquitination.

Shema, E., Tirosh, I., Aylon, Y., Huang, J., Ye, C., Moskovits, N., Raver-Shapira, N., Minsky, N., Pirngruber, J., Tarcic, G., et al. (2008). The histone H2B-specific ubiquitin ligase RNF20/hBRE1 acts as a putative tumor suppressor through selective regulation of gene expression. Genes Dev 22, 2664-2.

Shema, E., Kim, J., Roeder, R.G., and Oren, M. (2011). RNF20 inhibits TFIISfacilitated transcriptional elongation to suppress pro-oncogenic gene expression. Mol.Cell 42, 477-488.

Shi, X., Finkelstein, A., Wolf, A.J., Wade, P.A., Burton, Z.F., and Jaehning, J.A. (1996). Paf1p, an RNA polymerase II-associated factor in Saccharomyces cerevisiae, may have both positive and negative roles in transcription. Mol. Cell. Biol. 16, 669676.

Shin, H., Liu, T., Manrai, A.K., and Liu, X.S. (2009). CEAS: cis-regulatory element annotation system. Bioinforma. Oxf. Engl. 25, 2605-2606.

Shirwany, N.A., and Zou, M.-H. (2010). AMPK in cardiovascular health and disease. Acta Pharmacol. Sin. 31, 1075-1084.

Shukla, A., Stanojevic, N., Duan, Z., Shadle, T., and Bhaumik, S.R. (2006). Functional analysis of H2B-Lys-123 ubiquitination in regulation of H3-Lys-4 methylation and recruitment of RNA polymerase II at the coding sequences of several active genes in vivo. Jbiol Chem 281, 19045-19054.

Simon, J.A., and Lange, C.A. (2008). Roles of the EZH2 histone methyltransferase in cancer epigenetics. Mutat. Res. 647, 21-29. 
Smith, C.M., and Steitz, J.A. (1997). Sno storm in the nucleolus: new roles for myriad small RNPs. Cell 89, 669-672.

Smyth, G.K. (2004). Linear models and empirical Bayes methods for assessing differential expression in microarray experiments. Stat Appl Genet Mol Biol 3_ _, Article3.

Sobell, H.M. (1985). Actinomycin and DNA transcription. Proc. Natl. Acad. Sci. 82, $5328-5331$.

Song, J.J., and Lee, Y.J. (2007). Differential activation of the JNK signal pathway by UV irradiation and glucose deprivation. Cell. Signal. 19, 563-572.

Sowa, M.E., Bennett, E.J., Gygi, S.P., and Harper, J.W. (2009). Defining the human deubiquitinating enzyme interaction landscape. Cell 138, 389-403.

Spence, J., Gali, R.R., Dittmar, G., Sherman, F., Karin, M., and Finley, D. (2000). Cell cycle-regulated modification of the ribosome by a variant multiubiquitin chain. Cell 102, 67-76.

Sridhar, V.V., Kapoor, A., Zhang, K., Zhu, J., Zhou, T., Hasegawa, P.M., Bressan, R.A., and Zhu, J.K. (2007). Control of DNA methylation and heterochromatic silencing by histone H2B deubiquitination. Nature 447, 735-738.

Steger, D.J., Lefterova, M.I., Ying, L., Stonestrom, A.J., Schupp, M., Zhuo, D., Vakoc, A.L., Kim, J.-E., Chen, J., Lazar, M.A., et al. (2008). DOT1L/KMT4 recruitment and H3K79 methylation are ubiquitously coupled with gene transcription in mammalian cells. Mol. Cell. Biol. 28, 2825-2839.

Sterner, D.E., and Berger, S.L. (2000). Acetylation of histones and transcriptionrelated factors. Microbiol. Mol. Biol. Rev. Mmbr 64, 435-459.

Stock, J.K., Giadrossi, S., Casanova, M., Brookes, E., Vidal, M., Koseki, H., Brockdorff, N., Fisher, A.G., and Pombo, A. (2007). Ring1-mediated ubiquitination of $\mathrm{H} 2 \mathrm{~A}$ restrains poised RNA polymerase II at bivalent genes in mouse ES cells. Natcell Biol 9, 1428-1435.

Stokes, M.P., Rush, J., Macneill, J., Ren, J.M., Sprott, K., Nardone, J., Yang, V., Beausoleil, S.A., Gygi, S.P., Livingstone, M., et al. (2007). Profiling of UV-induced ATM/ATR signaling pathways. Proc.Natl.Acad.Sci.U.S.A 104, 19855-19860.

Stoykova, A.S., Dabeva, M.D., Dimova, R.N., and Hadjiolov, A.A. (1985). Ribosome biogenesis and nucleolar ultrastructure in neuronal and oligodendroglial rat brain cells. J. Neurochem. 45, 1667-1676.

Strahl, B.D., and Allis, C.D. (2000). The language of covalent histone modifications. Nature 403, 41-45.

Sun, L., and Chen, Z.J. (2004). The novel functions of ubiquitination in signaling. Curr. Opin. Cell Biol. 16, 119-126. 
Sun, Z.W., and Allis, C.D. (2002). Ubiquitination of histone H2B regulates H3 methylation and gene silencing in yeast. Nature 418, 104-108.

Taniguchi, K., Wada, M., Kohno, K., Nakamura, T., Kawabe, T., Kawakami, M., Kagotani, K., Okumura, K., Akiyama, S., and Kuwano, M. (1996). A human canalicular multispecific organic anion transporter (cMOAT) gene is overexpressed in cisplatin-resistant human cancer cell lines with decreased drug accumulation. Cancer Res. 56, 4124-4129.

Tanny, J.C., Erdjument-Bromage, H., Tempst, P., and Allis, C.D. (2007). Ubiquitylation of histone $\mathrm{H} 2 \mathrm{~B}$ controls RNA polymerase II transcription elongation independently of histone H3 methylation. Genes Dev 21, 835-847.

Taube, J.H., Herschkowitz, J.I., Komurov, K., Zhou, A.Y., Gupta, S., Yang, J., Hartwell, K., Onder, T.T., Gupta, P.B., Evans, K.W., et al. (2010). Core epithelial-tomesenchymal transition interactome gene-expression signature is associated with claudin-low and metaplastic breast cancer subtypes. Proc. Natl. Acad. Sci. 107, 15449-15454.

Thomas, J.O. (1999). Histone H1: location and role. Curr. Opin. Cell Biol. 11, 312317.

Tortoriello, D.V., Sidis, Y., Holtzman, D.A., Holmes, W.E., and Schneyer, A.L. (2001). Human follistatin-related protein: a structural homologue of follistatin with nuclear localization. Endocrinology 142, 3426-3434.

Towbin, H., Staehelin, T., and Gordon, J. (1979). Electrophoretic transfer of proteins from polyacrylamide gels to nitrocellulose sheets: procedure and some applications. Proc. Natl. Acad. Sci. U. S. A. 76, 4350-4354.

Turner, B.M. (1993). Decoding the nucleosome. Cell 75, 5-8.

Turner, B.M. (2000). Histone acetylation and an epigenetic code. Bioessays News Rev. Mol. Cell. Dev. Biol. 22, 836-845.

Urasaki, Y., Heath, L., and Xu, C.W. (2012). Coupling of glucose deprivation with impaired histone H2B monoubiquitination in tumors. Plos One 7, e36775.

Vassilev, L.T., Vu, B.T., Graves, B., Carvajal, D., Podlaski, F., Filipovic, Z., Kong, N., Kammlott, U., Lukacs, C., Klein, C., et al. (2004). In vivo activation of the p53 pathway by small-molecule antagonists of MDM2. Science 303, 844-848.

Vermeulen, M., Eberl, H.C., Matarese, F., Marks, H., Denissov, S., Butter, F., Lee, K.K., Olsen, J.V., Hyman, A.A., Stunnenberg, H.G., et al. (2010). Quantitative interaction proteomics and genome-wide profiling of epigenetic histone marks and their readers. Cell 142, 967-980.

Vethantham, V., Yang, Y., Bowman, C., Asp, P., Lee, J.H., Skalnik, D.G., and Dynlacht, B.D. (2012). Dynamic loss of H2B ubiquitylation without corresponding changes in H3K4 tri-methylation during myogenic differentiation. Molcell Biol. 
Vong, Q.P., Cao, K., Li, H.Y., Iglesias, P.A., and Zheng, Y. (2005). Chromosome alignment and segregation regulated by ubiquitination of survivin. Science 310 , 1499-1504.

Wang, H., Wang, L., Erdjument-Bromage, H., Vidal, M., Tempst, P., Jones, R.S., and Zhang, Y. (2004). Role of histone H2A ubiquitination in Polycomb silencing. Nature 431, 873-878.

Wang, Q., Liang, B., Shirwany, N.A., and Zou, M.-H. (2011). 2-Deoxy-D-Glucose Treatment of Endothelial Cells Induces Autophagy by Reactive Oxygen SpeciesMediated Activation of the AMP-Activated Protein Kinase. Plos One 6.

Wang, Z., Zang, C., Rosenfeld, J.A., Schones, D.E., Barski, A., Cuddapah, S., Cui, K., Roh, T.-Y., Peng, W., Zhang, M.Q., et al. (2008). Combinatorial patterns of histone acetylations and methylations in the human genome. Nat. Genet. 40, 897903.

Weake, V.M., and Workman, J.L. (2008). Histone ubiquitination: triggering gene activity. Mol.Cell 29, 653-663.

Weake, V.M., Lee, K.K., Guelman, S., Lin, C.H., Seidel, C., Abmayr, S.M., and Workman, J.L. (2008). SAGA-mediated H2B deubiquitination controls the development of neuronal connectivity in the Drosophila visual system. Embo J 27, 394-405.

Weake, V.M., Dyer, J.O., Seidel, C., Box, A., Swanson, S.K., Peak, A., Florens, L., Washburn, M.P., Abmayr, S.M., and Workman, J.L. (2011). Post-transcription initiation function of the ubiquitous SAGA complex in tissue-specific gene activation. Genes Dev. 25, 1499-1509.

Webster, I., Friedrich, S.O., Lochner, A., and Huisamen, B. (2010). AMP kinase activation and glut4 translocation in isolated cardiomyocytes. Cardiovasc. J. Afr. 21, 72-78.

Welt, C., Sidis, Y., Keutmann, H., and Schneyer, A. (2002). Activins, Inhibins, and Follistatins: From Endocrinology to Signaling. A Paradigm for the New Millennium. Exp. Biol. Med. 227, 724-752.

Wolffe, A.P., and Hayes, J.J. (1999). Chromatin disruption and modification. Nucleic Acids Res. 27, 711-720.

Wood, A., Krogan, N.J., Dover, J., Schneider, J., Heidt, J., Boateng, M.A., Dean, K., Golshani, A., Zhang, Y., Greenblatt, J.F., et al. (2003). Bre1, an E3 ubiquitin ligase required for recruitment and substrate selection of Rad6 at a promoter. Mol.Cell 11, 267-274.

Woodruff, T.K., and Mather, J.P. (1995). Inhibin, activin and the female reproductive axis. Annu. Rev. Physiol. 57, 219-244.

Wyce, A., Xiao, T., Whelan, K.A., Kosman, C., Walter, W., Eick, D., Hughes, T.R., Krogan, N.J., Strahl, B.D., and Berger, S.L. (2007). H2B ubiquitylation acts as a 
barrier to Ctk1 nucleosomal recruitment prior to removal by Ubp8 within a SAGArelated complex. Mol.Cell 27, 275-288.

Wysocki, R., Javaheri, A., Allard, S., Sha, F., Côté, J., and Kron, S.J. (2005). Role of Dot1-dependent histone $\mathrm{H} 3$ methylation in $\mathrm{G} 1$ and $\mathrm{S}$ phase DNA damage checkpoint functions of Rad9. Mol. Cell. Biol. 25, 8430-8443.

Xiao, B., Heath, R., Saiu, P., Leiper, F.C., Leone, P., Jing, C., Walker, P.A., Haire, L., Eccleston, J.F., Davis, C.T., et al. (2007). Structural basis for AMP binding to mammalian AMP-activated protein kinase. Nature 449, 496-500.

Xiao, T., Kao, C.-F., Krogan, N.J., Sun, Z.-W., Greenblatt, J.F., Osley, M.A., and Strahl, B.D. (2005a). Histone H2B ubiquitylation is associated with elongating RNA polymerase II. Mol. Cell. Biol. 25, 637-651.

Xiao, T., Kao, C.F., Krogan, N.J., Sun, Z.W., Greenblatt, J.F., Osley, M.A., and Strahl, B.D. (2005b). Histone H2B ubiquitylation is associated with elongating RNA polymerase II. Molcell Biol 25, 637-651.

Xu, H., Liu, Y.L., Yang, Y.M., and Dong, X.S. (2012). Knock-down of ubiquitinspecific protease 22 by micro-RNA interference inhibits colorectal cancer growth. Intjcolorectal Dis 27, 21-30.

Yamada, T., Yamaguchi, Y., Inukai, N., Okamoto, S., Mura, T., and Handa, H. (2006). P-TEFb-mediated phosphorylation of hSpt5 C-terminal repeats is critical for processive transcription elongation. Mol.Cell 21, 227-237.

Yang, X.-J. (2005). Multisite protein modification and intramolecular signaling. Oncogene 24, 1653-1662.

Yang, Y., Ludwig, R.L., Jensen, J.P., Pierre, S.A., Medaglia, M.V., Davydov, I.V., Safiran, Y.J., Oberoi, P., Kenten, J.H., Phillips, A.C., et al. (2005a). Small molecule inhibitors of HDM2 ubiquitin ligase activity stabilize and activate p53 in cells. Cancer Cell 7, 547-559.

Yang, Z., Yik, J.H., Chen, R., He, N., Jang, M.K., Ozato, K., and Zhou, Q. (2005b). Recruitment of P-TEFb for stimulation of transcriptional elongation by the bromodomain protein Brd4. Mol.Cell 19, 535-545.

Yoon, H.S., and Kim, H.A. (2004). Prologation of C-Jun N-terminal kinase is associated with cell death induced by tumor necrosis factor alpha in human chondrocytes. J. Korean Med. Sci. 19, 567-573.

Yu, J., Cao, Q., Mehra, R., Laxman, B., Yu, J., Tomlins, S.A., Creighton, C.J., Dhanasekaran, S.M., Shen, R., Chen, G., et al. (2007). Integrative genomics analysis reveals silencing of beta-adrenergic signaling by polycomb in prostate cancer. Cancer Cell 12, 419-431.

Zhang, Y. (2003). Transcriptional regulation by histone ubiquitination and deubiquitination. Genes Dev 17, 2733-2740. 
Zhang, F., and Yu, X. (2011). WAC, a functional partner of RNF20/RNF40, regulates histone H2B ubiquitination and gene transcription. Mol.Cell 41, 384-397.

Zhang, X.Y., Varthi, M., Sykes, S.M., Phillips, C., Warzecha, C., Zhu, W., Wyce, A., Thorne, A.W., Berger, S.L., and McMahon, S.B. (2008a). The putative cancer stem cell marker USP22 is a subunit of the human SAGA complex required for activated transcription and cell-cycle progression. Mol.Cell 29, 102-111.

Zhang, Y., Liu, T., Meyer, C.A., Eeckhoute, J., Johnson, D.S., Bernstein, B.E., Nusbaum, C., Myers, R.M., Brown, M., Li, W., et al. (2008b). Model-based Analysis of ChIP-Seq (MACS). Genome Biol. 9, R137.

Zhang, Y., Yao, L., Zhang, X., Ji, H., Wang, L., Sun, S., and Pang, D. (2011). Elevated expression of USP22 in correlation with poor prognosis in patients with invasive breast cancer. Jcancer Resclinoncol.

Zhang, Y., Foreman, O., Wigle, D.A., Kosari, F., Vasmatzis, G., Salisbury, J.L., van Deursen, J., and Galardy, P.J. (2012). USP44 regulates centrosome positioning to prevent aneuploidy and suppress tumorigenesis. J. Clin. Invest. 122, 4362-4374.

Zhao, Y., Lang, G., Ito, S., Bonnet, J., Metzger, E., Sawatsubashi, S., Suzuki, E., Le, G., Stunnenberg, H.G., Krasnov, A., et al. (2008). A TFTC/STAGA module mediates histone $\mathrm{H} 2 \mathrm{~A}$ and $\mathrm{H} 2 \mathrm{~B}$ deubiquitination, coactivates nuclear receptors, and counteracts heterochromatin silencing. Mol.Cell 29, 92-101.

Zheng, P.-P., Severijnen, L.-A., van der Weiden, M., Willemsen, R., and Kros, J.M. (2009). Cell proliferation and migration are mutually exclusive cellular phenomena in vivo: implications for cancer therapeutic strategies. Cell Cycle Georget. Tex 8, 950951.

Zhou, K., Kuo, W.H., Fillingham, J., and Greenblatt, J.F. (2009). Control of transcriptional elongation and cotranscriptional histone modification by the yeast BUR kinase substrate Spt5. Proc.Natl.Acad.Sci.U.S.A 106, 6956-6961.

Zhu, B., Zheng, Y., Pham, A.D., Mandal, S.S., Erdjument-Bromage, H., Tempst, P., and Reinberg, D. (2005). Monoubiquitination of human histone H2B: the factors involved and their roles in HOX gene regulation. Mol.Cell 20, 601-611. 


\section{Acknowledgements}

First of all, I would like to thank my supervisor Prof. Dr. Steven A. Johnsen for his intense support and guidance. I am thankful for his excellent scientific supervision, his inspirational and encouraging ideas and all his help. Moreover, I want to thank him for the friendly atmosphere he created in our group and for being available at all times.

I thank my thesis committee members Prof. Dr. Holger Reichardt and Prof. Dr. Dieter Kube for the inspiring discussions and their support throughout the thesis work. In addition, I appreciate Prof. Dr. Holger Reichardt for being the thesis second reviewer.

I also want to thank all members of the Department of Molecular Oncology for a nice and helpful working atmosphere. Many thanks go to Prof. Dr. Matthias Dobbelstein for giving me the opportunity to start my work in his department. Further, I am thankful to Prof. Dr. Klaus Pantel and the Department of Tumorbiology for the very warm welcome in our new lab after moving to Hamburg. In particular, I would like to thank all current and former members of the AG Johnsen for a wonderful working atmosphere and a great time spent together. Special thanks also go to my colleagues and friends Judith Pirngruber, Tanja Prenzel, Yvonne Begus-Nahrmann and Richard Schlegel.

For the practical performance and the initial analyses of the microarray data, I thank Dr. Gabriela Salinas-Riester, Susanne Luthin and Lennart Opitz. For the valuable statistical analyses I am very appreciative of Prof. Dr. Tim Beißbarth and Frank Kramer.

I would like to thank Prof. Dr. Hans Will and Dr. Yvonne Begus-Nahrmann for great help, discussions and valuable advices about thesis writing. Also many thanks go to Dr. Magali Hennion for her help with the preparation of the ChIP-seqencing data and their analysis.

My heartfelt thanks go to my parents, my brother and my friends. I want to thank them for their continuous support, their constant encouragement, their understanding and help and for being there for me at every step of the way. 


\section{Curriculum Vitae}

\section{THERESA GORSLER}

\section{PERSONAL DETAILS}

Date of birth:

$09 / 05 / 1986$

Nationality:

German

\section{EDUCATION}

07/2012 till now

$11 / 2009$ to $06 / 2012$

$10 / 2004$ to $09 / 2009$

$08 / 1996$ to $06 / 2004$
Ph.D. student at University Medical Center Hamburg-Eppendorf

Ph.D. student at University Medical Centre Göttingen

Friedrich-Schiller-Universität of Jena, Germany

Base studies of biology; final grade: 1,7

Main studies of biology with major subject in genetics $(1,3)$ and

minor subjects in microbiology $(2,3)$ and immunbiology $(1,3)$

Marie-Curie-Gymnasium in Worbis, Germany

German Abitur; final grade 2,3

\section{RESEARCH EXPERIENCE}

07/2012 until present

Department of Tumor Biology

Ph.D. thesis: Regulation of H2B monoubiquitination

Supervisor: Prof. Dr. Steve Johnsen

\section{$11 / 2009$ to $06 / 2012$}

Molecular Oncology, University Medical Centre Göttingen, Germany

Ph.D. thesis: Regulation of H2B monoubiquitination

Supervisor: Prof. Dr. Steve Johnsen

09/2008 to 08/2009

Institute of Human Genetics and Anthropology, Universitätsklinikum Jena

Jena, Germany

Diploma thesis project: The translocation of S100A11 after cellular stress stimuli and the effect on the cell cycle.

Supervisor: PD Dr. Christian Melle

03/2008 to $04 / 2008$

Institute of Molecular Genetics of Montpellier, part of CNRS

Montpellier, France

Research project at Dr. Michael Hahne's lab: Study of mechanisms controlling cellular proliferation and apoptosis induced by TRAIL in fibroblastic synoviocytes in Rheumatoid Arthritis. Supervisor: Dr. Rachel Audo 
$12 / 2007$ to $01 / 2008$

Institute of Human Genetics and Anthropology, Universitätsklinikum Jena

Jena, Germany

Department of Core Unit Chip Application

Placement at PD Dr. Ferdinand von Eggeling's lab: Proteomic characterisation of S100-proteins.

Supervisor: Dipl. Biol. Ulrike Murzik

$02 / 2007$ to $03 / 2007$

Leibniz-Institute for Age Research, Fritz-Lipmann-Institute (FLI)

Jena, Germany

Research project at Prof. Dr. Falk Weih's lab: Function of Traf in the activation of the alternative NF-kB pathways after LT $\beta$-receptor stimulation.

Supervisor: Dr. Marc Riemann

\section{RESEARCH SKILLS}

- Molecular biology: cloning, gel electrophoresis, PCR, real-time PCR, SDS-PAGE, Western Immunoblotting, Fluorescence Activated Cell Sorting (FACS), • Enzyme-Linked Immunosorbent Assay (ELISA), • Surface-Enhanced-Laser-Desorption/lonization - Time-Of-Flight - MassSpectrometry (SELDI-TOF-MS)

- Biochemistry: expression and purification of GST tagged recombinant proteins, pull-down, kinase assay, deubiquitination assay, immunoprecipitation, chromatin immunoprecipitation, nuclear run-on, immunofluorescence

- Cell Culture: mammalian, transfections, RNAi

- Microscopy: differential interference contrast, confocal

\section{PERSONAL SKILLS}

Computer Skills: MS Windows, MS Office, basic bioinformatics

\section{Languages}

- German: mother tongue

- Englisch: good knowledge

- French: basic knowledge

\section{PUBLICATIONS}

Gorsler T, Murzik U, Ulbricht T, Hentschel J, Hemmerich P, Melle C. (2010) DNA damageinduced translocation of S100A11 into the nucleus regulates cell proliferation. BMC Cell Biol. $17 ; 11: 100$.

Prenzel T, Begus-Nahrmann Y, Kramer F, Hennion M, Hsu C, Gorsler T, Hintermair C, Eick D, Kremmer E, Simons M, Beissbarth T, Johnsen SA. (2011) Estrogen-dependent gene transcription in human breast cancer cells relies upon proteasome-dependent monoubiquitination of histone H2B. Cancer Res. 1;71(17):5739-53 